\title{
Cohomological Hall algebra, exponential Hodge structures and motivic Donaldson-Thomas invariants
}

\author{
Maxim Kontsevich And Yan SoIBELman
}

To a quiver with potential we assign an algebra in the category of exponential mixed Hodge structures (the latter is also introduced in the paper). We compute the algebra (which we call Cohomological Hall algebra) for quivers without potential and study factorization properties of its Poincaré-Hilbert series in general case. As an application we obtain an alternative approach to our theory of motivic Donaldson-Thomas invariants of 3-dimensional Calabi-Yau categories and prove their integrality properties. We discuss the relationship of Cohomological Hall algebra with other mathematical structures including cluster algebras and ChernSimons theory.

1. Introduction

2. Cohomological Hall algebra of a quiver without potential

2.1. Stacks of representations and their cohomology

2.2. Multiplication

2.3. Proof of associativity

2.4. Explicit formula for the product

2.5. Example: quivers with one vertex

2.6. Symmetric case

2.7. Grading by the Heisenberg group and twisted graded algebras 
2.8. Non-symmetric example: quiver $\boldsymbol{A}_{2}$

2.9. Questions about other algebraic structures on $\mathcal{H}$

3. Generalization of COHA for smooth algebras

3.1. Cohomology theories

3.2. Smooth algebras

3.3. Cohomological Hall algebra for an $I$-bigraded smooth algebra

3.4. Square root of Tate motive and modified product

3.5. Algebra in the triangulated category of motives

3.6. Equivariant parameters in arrows and families of quivers

3.7. Complex cobordisms

4. Exponential Hodge structures and COHA for a smooth algebra with potential

4.1. Short synopsis

4.2. Exponential mixed Hodge structures

4.3. Realization functors for exponential mixed Hodge structures

4.4. Weight filtration in the exponential case

4.5. Cohomology of a variety with function

4.6. Étale case

4.7. Cohomological Hall algebra in the case of a smooth algebra with potential 
4.8. Example: linear potentials and cohomological dimension 2

5. Stability conditions, wall-crossing and motivic DT-series 280

5.1. Stability and Harder-Narasimhan filtration 280

5.2. Spectral sequence converging to $\mathcal{H}_{\gamma}$ 283

5.3. Generating series and quantum tori 285

5.4. Reformulation using cohomology with compact support

5.5. Classical limit

5.6. Examples 293

5.7. Mutations of quivers with potentials 298

6. Factorization systems and integrality of exponents 304

6.1. Admissible series and integrality properties 304

6.2. Admissibility of generating series and motivic Donaldson-Thomas invariants

6.3. Equivariant cohomology with respect to maximal tori

6.4. Factorization systems

6.5. Equivariant cohomology as a factorization system

6.6. From factorization systems to admissible series

6.7. Admissibility in the geometric case

6.8. One-dimensional factorization systems

6.9. Approximation by large integers and end of the proof of Theorems 6.1 and 6.2 
7. Critical COHA

7.1. Description of results

7.2. Vanishing cycles in the analytic case

7.3. Relation with the rapid decay cohomology

7.4. Vanishing cycles and monodromic Hodge structures

7.5. Rapid decay cohomology and monodromic Hodge structures

7.6. Definition of the critical COHA

7.7. Stability conditions and factorization of critical motivic DT-series

7.8. Integral identity

7.9. Factorization systems in the critical case

7.10. Comparison with motivic DT-invariants from $[34]$

8. Some speculations

8.1. Categorification of the critical COHA

8.2. Invariants of three-dimensional manifolds

References

\section{Introduction}

There is an old proposal in String theory (see [26]) which says that with a certain class of four-dimensional quantum theories with $N=2$ spacetime supersymmetry one should be able to associate an algebra graded by the charge lattice, called the algebra of BPS states. The goal of this paper is to 
propose a rigorous mathematical definition of an associative algebra, which is presumably related to the algebra of BPS states. We call it Cohomological Hall algebra (which we will sometimes abbreviate as COHA). Our construction can be applied to a wide class of situations, including representations of quivers (or more generally, smooth algebras) and arbitrary finitely presented algebras. It is also related to multi-matrix integrals. More general framework for our theory would be the class of ind-constructible three-dimensional Calabi-Yau categories (see [33]) whose objects form an ind-Artin stack.

Our definition is similar in the spirit to the conventional definition of the Hall algebra of an abelian category over a finite field. The main difference is that we use cohomology of moduli stacks of objects instead of constructible functions. In particular, Cohomological Hall algebra is different from the motivic Hall algebra introduced in [33]. In present paper virtual fundamental classes of proper (maybe singular) varieties classifying subrepresentations of a given representation serve as structure constants.

In the case of representations of a quiver, our construction is similar to the one of Lusztig (see [39]) of the canonical basis of the negative part of quantum enveloping algebra (see also a generalization to the affine case by Grojnowski [23]). An analogous idea appeared long ago in the work of Nakajima (see [43]), although in a slightly different context. That idea was used in [26] (see also [37]) in an (unsuccessful) attempt to define the associative algebra of BPS states. We remark that Lusztig's construction uses certain categories of equivariant $\mathcal{D}$-modules on representations spaces of quivers (or rather corresponding perverse sheaves). Our approach is based morally on the category of coherent sheaves, and it is more direct and elementary. It would be interesting to clarify the relation between quantum algebras and COHA.

In this paper, we present two different versions of Cohomological Hall algebra. The most simple one is the "off-shell" version, related to the socalled rapid decay cohomology of an algebraic variety endowed with function ("potential"). We develop a generalization of the theory of mixed Hodge structures in this "exponential" ${ }^{1}$ setting. An advantage of rapid decay cohomology is that one can use elementary topological methods and obtain very strong factorization properties for the motivic Donaldson-Thomas series

\footnotetext{
${ }^{1}$ Informally speaking, we study integrals of the form $\int \exp (f)$ where $f$ is the potential.
} 
of the corresponding $\mathrm{COHA}^{2}$. As a corollary, we define integer numbers, which (hypothetically) count BPS states, and satisfy wall-crossing formulas from [33].

Another goal of the paper is to give an alternative approach to the theory of motivic Donaldson-Thomas invariants introduced in [33]. Having a quiver with potential one can define an ind-constructible $3 C Y$ category. Its $t$-structure has the heart consisting of finite-dimensional representations of the quiver, which are critical points of the potential. This is the framework in which the theory from [33] can be applied. In order to compare the invariants from the loc. cit. with those introduced in present paper one needs an "onshell" version of Cohomological Hall algebra defined in terms of sheaves of vanishing cycles. The corresponding cohomology theory is related to asymptotic expansions of exponential integrals in the formal neighborhood of the critical locus of the potential. We call it the critical cohomology. We prove in Section 7 that the motivic DT-series for the "critical COHA" essentially coincides with the one introduced in [33]. In order to define critical COHA we need several foundational results concerning sheaves of vanishing cycles. In particular, we need a version of Thom-Sebastiani theorem for mixed Hodge modules (proven by Saito in [50]) as well as the integral identity from [33], which we prove here in the sheaf-theoretic framework.

As we pointed out above, we expect that the approach of this paper can be generalized to a wider class of three-dimensional Calabi-Yau categories (after a choice of generator any such category can be in a sense described "locally" by a quiver with potential). Restriction to the case of a smooth algebra with potential allows us to avoid a discussion of some technical difficulties of the general definition from [33]. In particular, there is no problem with orientation data. Furthermore, we can prove the integrality property of generating series which is in a sense stronger than the one conjectured in [33]. The new integrality property has a nice algebraic meaning for our Cohomological Hall algebra as a kind of Poincaré-Birkhoff-Witt theorem. The proof is based on the consideration of equivariant cohomology with respect to the action of maximal tori.

Summarizing, in all cases we define a graded vector space $\mathcal{H}$ which is:

(i) An associative algebra (more precisely, with a twisted associativity in the motivic setting). The algebra itself depends on a quiver with potential, not on a stability function.

\footnotetext{
${ }^{2}$ Motivic DT-series are the generating series for the Serre polynomials of graded components of COHA.
} 
(ii) For given stability function, the motivic DT-series of the algebra admits a factorization (in the clockwise order) into a product of factors over all possible slopes. Each factor is defined in terms of the moduli space of semistable objects with a given slope. This factorization property is a consequence of an existence of a spectral sequence converging to the graded components of $\mathcal{H}$.

(iii) The motivic DT-series admits an infinite product expression in terms of standard $q$-special functions (basically, shifted quantum dilogarithms) with integer exponents (the latter should be called "refined DT-invariants" by analogy with "refined BPS invariants" in physics).

This product structure is based on the theory of factorization systems introduced in this paper. The reader should keep in mind all the goals (i)-(iii) while reading about various generalizations and ramifications, which we discuss in this paper.

Finally, we remark that it would be highly desirable to relate the classical limits of our motivic Donaldson-Thomas invariants with the invariants introduced by Joyce and Song (see [28]). More precisely, we expect that the category of coherent sheaves used in the loc. cit. carries orientation data and our motivic DT-invariants (see Section 6) give in the classical limit the invariants introduced there.

Let us briefly discuss the content of the paper.

Section 2 is devoted to the definition of COHA in the case of a quiver without potential. We also present the formula for the product based on the toric localization technique and apply it to several examples. We observe that the canonical grading of COHA by the "charge lattice" can be extended to a grading by the Heisenberg group which is non-commutative.

Section 3 is devoted to various generalizations of COHA. In particular, the path algebra of a quiver with the set of vertices $I$ can be replaced by an I-bigraded algebra, which is smooth in the sense of Cuntz and Quillen. We also remark that there should be a natural generalization of COHA as an $A_{\infty}$-algebra in the dg-enhancement of the Voevodsky category of mixed motives.

In Section 4, we discuss COHA in the case of smooth $I$-bigraded algebra with potential. The definition is based on the properties of the category EMHS of exponential mixed Hodge structures introduced in that section. Objects of EMHS admit weight filtration which allows us to introduce Serre polynomials of graded components of COHA. There are several realizations of COHA depending on a choice of cohomology theory (i.e., a cohomology 
functor from the tensor category EMHS to a Tannakian category). For example, one can choose rapid decay cohomology theory, which corresponds to the Betti realization of EMHS. Another choice is the de Rham realization. The comparison between Betti and de Rham realizations is related to the theory of matrix integrals, since the conventional pairing between algebraic differential forms and algebraic cycles (period map) now becomes a pairing between "exponential forms" of the type $\exp (f) \alpha$ and non-compact cycles $C$ such that the restriction $\operatorname{Re}(f)_{\mid C}$ is bounded. We end Section 4 with a result that sometimes allows us to reduce computations of COHA from (cohomological) dimension 3 to dimension 2 .

In Section 5, we introduce the notion of stability condition and discuss the corresponding spectral sequence converging to COHA. We also introduce motivic DT-series of a smooth $I$-bigraded algebra with potential and prove the Factorization formula (a.k.a. wall-crossing formula) similar to the one from [33]. In the case of a quiver with potential, we study how the DT-series changes with respect to a mutation (the result agrees with the one from Section 8 of [33]).

In Section 6, we introduce motivic DT-invariants and discuss the product structure of motivic DT-series. Integrality of the exponents in the product formula (refined DT-invariants), see Section 6.1, is a non-trivial fact which follows from the so-called admissibility property of the motivic DTseries. Corresponding technique of factorization systems and proofs are contained in Section 6. Although COHA and motivic DT-series do not depend on the stability function (a.k.a. central charge), the motivic DT-invariants do.

Section 7 is devoted to the critical COHA defined in terms of vanishing cycles functor. The corresponding cohomology theory is called critical cohomology. Some important properties of cohomology theories used in the previous sections do not hold for critical cohomology, e.g., Thom isomorphism. As a result, the very definition of COHA requires much more work. The situation is in a sense similar to the categorical one from [33]. In particular, we need to prove in Section 7.8 the integral identity sketched in the loc. cit. Importance of the critical COHA is explained in Section 7.10, where we prove that the corresponding motivic DT-series is basically the same as the "categorical one" from [33].

In the last Section 8 we discuss two speculative applications: categorification of COHA and motivic DT-invariants arising in the Chern-Simons theory with complex gauge group. There are many more applications and speculations, which we decided not to include in order to save the space. 


\section{Cohomological Hall algebra of a quiver without potential}

\subsection{Stacks of representations and their cohomology}

Let us fix a finite quiver $Q$, with the set $I$ of vertices, and $a_{i j} \in \mathbb{Z}_{\geqslant 0}$ arrows from $i$ to $j$ for $i, j \in I$. For any dimension vector

$$
\gamma=\left(\gamma^{i}\right)_{i \in I} \in \mathbb{Z}_{\geqslant 0}^{I}
$$

consider the space of representations of $Q$ in complex coordinate vector spaces of dimensions $\left(\gamma^{i}\right)_{i \in I}$

$$
\mathrm{M}_{\gamma}=\mathrm{M}_{\gamma}^{Q} \simeq \prod_{i, j \in I} \mathbb{C}^{a_{i j} \gamma^{i} \gamma^{j}}
$$

endowed with the action by conjugation of a complex algebraic group

$$
\mathrm{G}_{\gamma}:=\prod_{i \in I} \mathrm{GL}\left(\gamma^{i}, \mathbb{C}\right)
$$

The quotient stack $\mathrm{M}_{\gamma} / \mathrm{G}_{\gamma}$ is the stack of representations of $Q$ with dimension vector $\gamma$. We will consider the cohomology of this stack, i.e., the equivariant cohomology of $\mathrm{M}_{\gamma}$ with $\mathrm{G}_{\gamma}$-action. We use the standard model

$$
\operatorname{Gr}\left(d, \mathbb{C}^{\infty}\right):=\lim _{\longrightarrow} \operatorname{Gr}\left(d, \mathbb{C}^{N}\right), N \rightarrow+\infty
$$

of the classifying space of $\operatorname{GL}(d, \mathbb{C})$ for $d \geqslant 0$, and define

$$
\mathrm{BG}_{\gamma}:=\prod_{i \in I} \operatorname{BGL}\left(\gamma^{i}, \mathbb{C}\right)=\prod_{i \in I} \operatorname{Gr}\left(\gamma^{i}, \mathbb{C}^{\infty}\right)
$$

Stack $\mathrm{M}_{\gamma} / \mathrm{G}_{\gamma}$ gives the universal family over $\mathrm{BG}_{\gamma}$

$$
\mathrm{M}_{\gamma}^{\mathrm{univ}}:=\left(\mathrm{EG}_{\gamma} \times \mathrm{M}_{\gamma}\right) / \mathrm{G}_{\gamma}
$$

where $\mathrm{EG}_{\gamma} \rightarrow \mathrm{BG}_{\gamma}$ is the standard universal $\mathrm{G}_{\gamma}$-bundle.

We introduce a $\mathbb{Z}_{\geqslant 0}^{I}$-graded abelian group

$$
\mathcal{H}:=\oplus_{\gamma} \mathcal{H}_{\gamma}
$$

where each component is defined as an equivariant cohomology

$$
\mathcal{H}_{\gamma}:=H_{\mathrm{G}_{\gamma}}^{\bullet}\left(\mathrm{M}_{\gamma}\right):=H^{\bullet}\left(\mathrm{M}_{\gamma}^{\text {univ }}\right)=\oplus_{n \geqslant 0} H^{n}\left(\mathrm{M}_{\gamma}^{\text {univ }}\right)
$$


Here by cohomology of a complex algebraic variety (or of an inductive limit of varieties) we mean the usual (Betti) cohomology with coefficients in $\mathbb{Z}$.

Notice that the natural Hodge structure on $H^{n}\left(\mathrm{M}_{\gamma}^{\text {univ }}\right)$ is pure of weight $n$, i.e., incidentally the cohomological degree coincides with the weight.

\subsection{Multiplication}

Fix any $\gamma_{1}, \gamma_{2} \in \mathbb{Z}_{\geqslant 0}^{I}$ and denote $\gamma:=\gamma_{1}+\gamma_{2}$. Denote by $\mathbf{M}_{\gamma_{1}, \gamma_{2}}$ the space of representations of $Q$ in coordinate spaces of dimensions $\left(\gamma_{1}^{i}+\gamma_{2}^{i}\right)_{i \in I}$ such that the standard coordinate subspaces of dimensions $\left(\gamma_{1}^{i}\right)_{i \in I}$ form a subrepresentation. Obviously $M_{\gamma_{1}, \gamma_{2}}$ is an affine space, and also a closed subspace of $\mathrm{M}_{\gamma}$. The group $\mathrm{G}_{\gamma_{1}, \gamma_{2}} \subset \mathrm{G}_{\gamma}$ consisting of transformations preserving subspaces $\left(\mathbb{C}^{\gamma_{1}^{i}} \subset \mathbb{C}^{\gamma^{i}}\right)_{i \in I}$ (i.e., the group of block upper-triangular matrices), acts on $\mathbf{M}_{\gamma_{1}, \gamma_{2}}$. In what follows, we will use the model of $\mathbf{B G}_{\gamma_{1}, \gamma_{2}}$ which is the total space of the bundle over $\mathrm{BG}_{\gamma}$ with the fiber $\mathrm{G}_{\gamma} / \mathrm{G}_{\gamma_{1}, \gamma_{2}}$.

Let us consider a morphism

$$
\mathrm{m}_{\gamma_{1}, \gamma_{2}}: \mathcal{H}_{\gamma_{1}} \otimes \mathcal{H}_{\gamma_{2}} \rightarrow \mathcal{H}_{\gamma}=\mathcal{H}_{\gamma_{1}+\gamma_{2}},
$$

which is the composition of the multiplication morphism (which becomes Künneth isomorphism after the extension of coefficients for cohomology from $\mathbb{Z}$ to $\mathbb{Q}$ )

$$
\otimes: H_{\mathrm{G}_{\gamma_{1}}}^{\bullet}\left(\mathrm{M}_{\gamma_{1}}\right) \otimes H_{\mathrm{G}_{\gamma_{2}}}^{\bullet}\left(\mathrm{M}_{\gamma_{2}}\right) \rightarrow H_{\mathrm{G}_{\gamma_{1}} \times \mathrm{G}_{\gamma_{2}}}^{\bullet}\left(\mathrm{M}_{\gamma_{1}} \times \mathrm{M}_{\gamma_{2}}\right),
$$

and of the following sequence of three morphisms:

$$
H_{\mathrm{G}_{\gamma_{1}} \times \mathrm{G}_{\gamma_{2}}}^{\bullet}\left(\mathrm{M}_{\gamma_{1}} \times \mathrm{M}_{\gamma_{2}}\right) \stackrel{\cong}{\rightrightarrows} H_{\mathrm{G}_{\gamma_{1}, \gamma_{2}}}^{\bullet}\left(\mathrm{M}_{\gamma_{1}, \gamma_{2}}\right) \rightarrow H_{\mathrm{G}_{\gamma_{1}, \gamma_{2}}}^{\bullet+2 c_{1}}\left(\mathrm{M}_{\gamma}\right) \rightarrow H_{\mathrm{G}_{\gamma}}^{\bullet+2 c_{1}+2 c_{2}}\left(\mathrm{M}_{\gamma}\right),
$$

where

(1) the first arrow is an isomorphism induced by natural projections of spaces and groups, inducing homotopy equivalences

$$
\mathrm{M}_{\gamma_{1}} \times \mathrm{M}_{\gamma_{2}} \approx \mathrm{M}_{\gamma_{1}, \gamma_{2}}, \quad \mathrm{G}_{\gamma_{1}} \times \mathrm{G}_{\gamma_{2}} \approx \mathrm{G}_{\gamma_{1}, \gamma_{2}} ;
$$

(2) the second arrow is the pushforward map associated with the closed $\mathrm{G}_{\gamma_{1}, \gamma_{2}}$-equivariant embedding $\mathrm{M}_{\gamma_{1}, \gamma_{2}} \hookrightarrow \mathrm{M}_{\gamma}$ of complex manifolds;

(3) the third arrow is the pushforward map associated with the fundamental class of compact complex manifold $\mathrm{G}_{\gamma} / \mathrm{G}_{\gamma_{1}, \gamma_{2}}$, which is the product of Grassmannians $\prod_{i \in I} \operatorname{Gr}\left(\gamma_{1}^{i}, \mathbb{C}^{\gamma^{i}}\right)$. 
Shifts in the cohomological degrees are given by

$$
c_{1}=\operatorname{dim}_{\mathbb{C}} \mathrm{M}_{\gamma}-\operatorname{dim}_{\mathbb{C}} \mathrm{M}_{\gamma_{1}, \gamma_{2}}, \quad c_{2}=-\operatorname{dim}_{\mathbb{C}} \mathrm{G}_{\gamma} / \mathrm{G}_{\gamma_{1}, \gamma_{2}}
$$

We endow $\mathcal{H}$ with a product $\mathrm{m}: \mathcal{H} \otimes \mathcal{H} \rightarrow \mathcal{H}, \mathrm{m}:=\sum_{\gamma_{1}, \gamma_{2}} \mathrm{~m}_{\gamma_{1}, \gamma_{2}}$.

Theorem 2.1. The product $\mathrm{m}$ on $\mathcal{H}$ is associative.

Moreover, it is straightforward to see that the element $1_{\mathrm{M}_{0}} \in \mathcal{H}_{0}$ is the unit for the product. The proof of Theorem 2.1 will be given in Section 2.3.

Definition 2.1. The associative unital $\mathbb{Z}_{\geqslant 0}^{I}$-graded algebra $\mathcal{H}$ with the product $\mathrm{m}$ is called the Cohomological Hall algebra associated with the quiver $Q$.

The multiplication does not preserve the cohomological grading. The shift is given by

$$
2\left(c_{1}+c_{2}\right)=-2 \chi_{Q}\left(\gamma_{1}, \gamma_{2}\right)
$$

where

$$
\chi_{Q}\left(\gamma_{1}, \gamma_{2}\right):=-\sum_{i, j \in I} a_{i j} \gamma_{1}^{j} \gamma_{2}^{i}+\sum_{i \in I} \gamma_{1}^{i} \gamma_{2}^{i}
$$

is the Euler form on the $K_{0}$ group of the category of finite-dimensional representations of $Q$ :

$$
\chi_{Q}\left(\gamma_{1}, \gamma_{2}\right)=\operatorname{dim} \operatorname{Hom}\left(E_{1}, E_{2}\right)-\operatorname{dim} \operatorname{Ext}^{1}\left(E_{1}, E_{2}\right)=\chi\left(\operatorname{Ext}^{\bullet}\left(E_{1}, E_{2}\right)\right),
$$

where $E_{1}$ and $E_{2}$ are arbitrary representations of $Q$, of dimension vectors $\gamma_{1}$ and $\gamma_{2}$.

The multiplication in $\mathcal{H}$ can be defined in a slightly different way, which is maybe more intuitively clear. Namely, consider the following map of manifolds endowed with $\mathrm{G}_{\gamma}$-action:

$$
\pi: \mathrm{Gr}_{\gamma_{1}, \gamma}:=\mathrm{G}_{\gamma} \times_{\mathrm{G}_{\gamma_{1}, \gamma_{2}}} \mathrm{M}_{\gamma_{1}, \gamma_{2}} \rightarrow \mathrm{M}_{\gamma}, \quad(g, m) \mapsto g m
$$

Notice that $\operatorname{dim} \mathrm{Gr}_{\gamma_{1}, \gamma}-\operatorname{dim} \mathrm{M}_{\gamma}=\chi_{Q}\left(\gamma_{1}, \gamma_{2}\right)$. The map $\pi$ is proper, hence it induces the pushforward (Gysin) morphism:

$$
\pi_{*}: H_{\mathrm{G}_{\gamma}}^{\bullet}\left(\mathrm{Gr}_{\gamma_{1}, \gamma}\right) \rightarrow H_{\mathrm{G}_{\gamma}}^{\bullet-2 \chi_{Q}\left(\gamma_{1}, \gamma_{2}\right)}\left(\mathrm{M}_{\gamma}\right)
$$


There are two natural $\mathrm{G}_{\gamma}$-equivariant bundles of representations of $Q$ of dimension vectors $\gamma_{1}$ and $\gamma_{2}$ on $\operatorname{Gr}_{\gamma_{1}, \gamma}$. Combining the pullback morphism

$$
H_{\mathrm{G}_{\gamma_{1}}}^{\bullet}\left(\mathrm{M}_{\gamma_{1}}\right) \otimes H_{\mathrm{G}_{\gamma_{2}}}^{\bullet}\left(\mathrm{M}_{\gamma_{2}}\right) \rightarrow H_{\mathrm{G}_{\gamma}}^{\bullet}\left(\mathrm{Gr}_{\gamma_{1}, \gamma}\right)
$$

with $\pi_{*}$, we obtain an equivalent definition of the multiplication morphism.

One can make still another reformulation using language of stacks. The natural morphism of stacks

$$
\mathrm{M}_{\gamma_{1}, \gamma_{2}} / \mathrm{G}_{\gamma_{1}, \gamma_{2}} \rightarrow \mathrm{M}_{\gamma} / \mathrm{G}_{\gamma}
$$

is a proper morphism of smooth Artin stacks, hence it induces the pushforward map on cohomology. Combining it with the pullback by the homotopy equivalence $\mathrm{M}_{\gamma_{1}} / \mathrm{G}_{\gamma_{1}} \times \mathrm{M}_{\gamma_{2}} / \mathrm{G}_{\gamma_{2}} \leftarrow \mathrm{M}_{\gamma_{1}, \gamma_{2}} / \mathrm{G}_{\gamma_{1}, \gamma_{2}}$, we obtain $\mathrm{m}_{\gamma_{1}, \gamma_{2}}$.

\subsection{Proof of associativity}

For given $\gamma_{1}, \gamma_{2}, \gamma_{3} \in \mathbb{Z}_{\geqslant 0}^{I}$, consider the following commutative diagram where we omit for the convenience shifts in the cohomological degree:

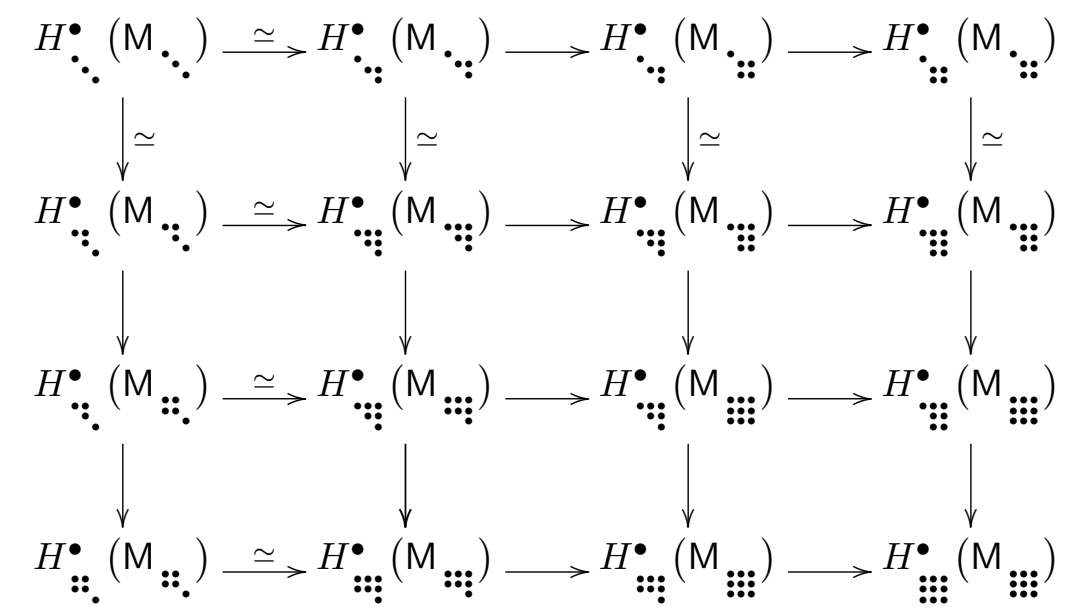

Here we use a shorthand notation for various equivariant cohomology groups, with dots denoting non-trivial blocks for operators in $\oplus_{i}\left(\mathbb{C}_{1}^{i}+\gamma_{2}^{i}+\gamma_{3}^{i}\right)$, e.g.,

$$
H_{\because .}^{\bullet}\left(\mathrm{M}_{: \text {. }}\right)=H_{\mathrm{G}_{\gamma_{1}, \gamma_{2}} \times \mathrm{G}_{\gamma_{3}}}^{\bullet}\left(\mathrm{M}_{\gamma_{1}+\gamma_{2}} \times \mathrm{M}_{\gamma_{3}}\right) \text {. }
$$

Traveling first down then right, we obtain the map $a_{1} \otimes a_{2} \otimes a_{3} \mapsto\left(a_{1} \cdot a_{2}\right)$. $a_{3}$, whereas traveling first right then down we get $a_{1} \otimes a_{2} \otimes a_{3} \mapsto a_{1} \cdot\left(a_{2}\right.$. $\left.a_{3}\right)$. The associativity of the product on $\mathcal{H}$ is proven. 


\subsection{Explicit formula for the product}

Here we calculate the product on $\mathcal{H}$ using the toric localization formula.

First of all, for any $\gamma$ the abelian group $\mathcal{H}_{\gamma}$ is the cohomology of the classifying space $\mathrm{BG}_{\gamma}$, as the manifold $\mathrm{M}_{\gamma}$ is contractible. It is well known that $H^{\bullet}(\operatorname{BGL}(n, \mathbb{C}))$ can be canonically identified with the algebra of symmetric polynomials with integer coefficients in $n$ variables of degree +2 for any $n \geqslant 0$, via the embedding

$$
H^{\bullet}(\operatorname{BGL}(n, \mathbb{C})) \hookrightarrow H^{\bullet}\left(\mathrm{B}\left(\mathbb{C}^{\times}\right)^{n}\right) \simeq \mathbb{Z}\left[x_{1}, \ldots, x_{n}\right]
$$

induced by the diagonal embedding $\left(\mathbb{C}^{\times}\right)^{n} \hookrightarrow \operatorname{GL}(n, \mathbb{C})$. Therefore, $\mathcal{H}_{\gamma}$ is realized as the abelian group of polynomials in variables $\left(x_{i, \alpha}\right)_{i \in I, \alpha \in\left\{1, \ldots, \gamma^{i}\right\}}$ symmetric under the group $\prod_{i \in I} \mathrm{Sym}_{\gamma^{i}}$ of permutations preserving index $i$ and permuting index $\alpha$.

Theorem 2.2. The product $f_{1} \cdot f_{2}$ of elements $f_{i} \in \mathcal{H}_{\gamma_{i}}, i=1,2$ is given by the symmetric function $g\left(\left(x_{i, \alpha}\right)_{i \in I, \alpha \in\left\{1, \ldots, \gamma^{i}\right\}}\right)$, where $\gamma:=\gamma_{1}+\gamma_{2}$, obtained from the following function in variables $\left(x_{i, \alpha}^{\prime}\right)_{i \in I, \alpha \in\left\{1, \ldots, \gamma_{1}^{i}\right\}}$ and $\left(x_{i, \alpha}^{\prime \prime}\right)_{i \in I, \alpha \in\left\{1, \ldots, \gamma_{2}^{i}\right\}}$ :

$$
f_{1}\left(\left(x_{i, \alpha}^{\prime}\right)\right) f_{2}\left(\left(x_{i, \alpha}^{\prime \prime}\right)\right) \frac{\prod_{i, j \in I} \prod_{\alpha_{1}=1}^{\gamma_{1}^{i}} \prod_{\alpha_{2}=1}^{\gamma_{2}^{j}}\left(x_{j, \alpha_{2}}^{\prime \prime}-x_{i, \alpha_{1}}^{\prime}\right)^{a_{i j}}}{\prod_{i \in I} \prod_{\alpha_{1}=1}^{\gamma_{1}^{i}} \prod_{\alpha_{2}=1}^{\gamma_{2}^{i}}\left(x_{i, \alpha_{2}}^{\prime \prime}-x_{i, \alpha_{1}}^{\prime}\right)}
$$

by taking the sum over all shuffles for any given $i \in I$ of the variables $x_{i, \alpha}^{\prime}, x_{i, \alpha}^{\prime \prime}$ (the sum is over $\prod_{i \in I}\left(\begin{array}{c}\gamma^{i} \\ \gamma_{1}^{i}\end{array}\right)$ shuffles).

Proof. The pushforward map

$$
H^{\bullet}\left(B \mathrm{G}_{\gamma_{1}} \times B \mathrm{G}_{\gamma_{2}}\right) \simeq H_{\mathrm{G}_{\gamma_{1}, \gamma_{2}}}^{\bullet}\left(\mathrm{M}_{\gamma_{1}, \gamma_{2}}\right) \rightarrow H_{\mathrm{G}_{\gamma_{1}, \gamma_{2}}}^{\bullet+2 c_{1}}\left(\mathrm{M}_{\gamma}\right) \simeq H^{\bullet+2 c_{1}}\left(B \mathrm{G}_{\gamma_{1}} \times B \mathrm{G}_{\gamma_{2}}\right)
$$

is just the multiplication by the equivariant Euler class

$$
e_{\gamma_{1}, \gamma_{2}} \in H^{2 c_{1}}\left(B \mathrm{G}_{\gamma_{1}} \times B \mathrm{G}_{\gamma_{2}}\right), \quad c_{1}=\sum_{i, j \in I} a_{i j} \gamma_{1}^{i} \gamma_{2}^{j}
$$

of the fiber at $0 \in \mathrm{M}_{\gamma_{1}, \gamma_{2}}$ of the normal bundle to $\mathrm{M}_{\gamma_{1}, \gamma_{2}} \subset \mathrm{M}_{\gamma}$.

The formula for the multiplication implies that $f_{1} \cdot f_{2}$ for $f_{i} \in \mathcal{H}_{\gamma_{i}}, i=$ 1,2 is obtained in the following way. First, we consider two canonical $\mathrm{G}_{\gamma_{i}}{ }^{-}$ bundles over $\mathrm{G}_{\gamma} / \mathrm{G}_{\gamma_{1}, \gamma_{2}}$ for $i=1,2$, which are equivariant with respect to $\mathrm{G}_{\gamma}$-action. Then we take the product of their $\mathrm{G}_{\gamma}$-equivariant characteristic 
classes corresponding to symmetric polynomials $f_{1}, f_{2}$, multiply the result by the $\mathrm{G}_{\gamma}$-equivariant class corresponding to $e_{\gamma_{1}, \gamma_{2}}$, and then take the integral over the $\mathrm{G}_{\gamma}$-equivariant fundamental class of $\mathrm{G}_{\gamma} / \mathrm{G}_{\gamma_{1}, \gamma_{2}}=\prod_{i \in I} \operatorname{Gr}\left(\gamma_{1}^{i}, \mathbb{C}^{\gamma^{i}}\right)$. The result is an element of the cohomology ring of $\mathrm{BG}_{\gamma}$.

We can use the equivariant cohomology with respect to the maximal torus $T_{\gamma}$ of $G_{\gamma}$, instead of $G_{\gamma}$. The set of fixed points of $T_{\gamma}$ on $G_{\gamma} / G_{\gamma_{1}, \gamma_{2}}$ is the set of collections of coordinate subspaces, i.e., exactly the set of shuffles of variables $x_{i, \alpha}^{\prime}$ and $x_{i, \alpha}^{\prime \prime}$. The numerator in the formula in the theorem is the product of $\mathrm{T}_{\gamma}$-weights corresponding to the class $e_{\gamma_{1}, \gamma_{2}}$. The denominator is the product of $T_{\gamma}$-weights in the tangent space of $G_{\gamma} / G_{\gamma_{1}, \gamma_{2}}$. The classical fixed point formula of Bott gives the result.

Remark 2.1. The above algebra is a special case of Feigin-Odesskii shuffle algebra (see $[17,15])$.

\subsection{Example: quivers with one vertex}

Let $Q=Q_{d}$ be now a quiver with just one vertex and $d \geqslant 0$ loops. Then the product formula from the previous section specializes to

$$
\begin{aligned}
& \left(f_{1} \cdot f_{2}\right)\left(x_{1}, \ldots, x_{n+m}\right) \\
& :=\sum_{\substack{i_{1}<\ldots<i_{n} \\
j_{1}<\cdots<j_{m} \\
\left\{i_{1}, \ldots, i_{n}, j_{1}, \ldots, j_{m}\right\} \\
=\{1, \ldots, n+m\}}} f_{1}\left(x_{i_{1}}, \ldots, x_{i_{n}}\right) f_{2}\left(x_{j_{1}}, \ldots, x_{j_{m}}\right)\left(\prod_{k=1}^{n} \prod_{l=1}^{m}\left(x_{j_{l}}-x_{i_{k}}\right)\right) d-1
\end{aligned}
$$

for symmetric polynomials, where $f_{1}$ has $n$ variables, and $f_{2}$ has $m$ variables. The product $f_{1} \cdot f_{2}$ is a symmetric polynomial in $n+m$ variables.

We introduce a double grading on algebra $\mathcal{H}$, by declaring that a homogeneous symmetric polynomial of degree $k$ in $n$ variables has bigrading $\left(n, 2 k+(1-d) n^{2}\right)$. Equivalently, one can shift the cohomological grading in $H^{\bullet}(\operatorname{BGL}(n, \mathbb{C}))$ by $\left[(d-1) n^{2}\right]$.

It follows directly from the product formula that the bigraded algebra is commutative for odd $d$, and supercommutative for even $d$. The parity in this algebra is given by the parity of the shifted cohomological degree.

It is easy to see that for $d=0$ the algebra $\mathcal{H}$ is an exterior algebra (i.e. Grassmann algebra) generated by odd elements $\psi_{1}, \psi_{3}, \psi_{5}, \ldots$ of bidegrees 
$(1,1),(1,3),(1,5), \ldots$ Generators $\left(\psi_{2 i+1}\right)_{i \geqslant 0}$ correspond to the additive generators $\left(x^{i}\right)_{i \geqslant 0}$ of

$$
H^{\bullet}\left(\mathbb{C} P^{\infty}\right)=H^{\bullet}(\operatorname{BGL}(1, \mathbb{C})) \simeq \mathbb{Z}[x] \simeq \mathbb{Z}\left[x_{1}\right]
$$

A monomial in the exterior algebra

$$
\psi_{2 i_{1}+1} \cdots \cdots \psi_{2 i_{n}+1} \in \mathcal{H}_{n, \sum_{k=1}^{n}\left(2 i_{k}+1\right)}, \quad 0 \leqslant i_{1}<\cdots<i_{n}
$$

corresponds to the Schur symmetric function $s_{\lambda}\left(x_{1}, \ldots, x_{n}\right)$, where

$$
\lambda=\left(i_{n}+(1-n), i_{n-1}+(2-n), \ldots, i_{1}\right)
$$

is a partition of length $\leqslant n$.

Similarly, for $d=1$ algebra $\mathcal{H}$ is isomorphic (after tensoring by $\mathbb{Q}$ ) to the algebra of symmetric functions in infinitely many variables, and it is a polynomial algebra generated by even elements $\phi_{0}, \phi_{2}, \phi_{4}, \ldots$ of bidegrees $(1,0),(1,2),(1,4), \ldots$ Again, the generators $\left(\phi_{2 i}\right)_{i \geqslant 0}$ correspond to the additive generators $\left(x^{i}\right)_{i \geqslant 0}$ of $H^{\bullet}\left(\mathbb{C} P^{\infty}\right) \simeq \mathbb{Z}[x]$. For any $n \geqslant 0$, the collection of all monomials of degree $n$ in generators $\left(\phi_{2 i}\right)_{i \geqslant 0}$ coincides (up to non-zero factors) with the monomial basis of the algebra of symmetric functions in $n$ variables.

Notice that the underlying additive group of the algebra $\mathcal{H}$ is equal to $\left.\oplus_{n \geqslant 0} H^{\bullet}(\operatorname{BGL}(n, \mathbb{C}))\right)$ and hence does not depend on $d$. The isomorphism (after tensoring by $\mathbb{Q}$ ) between the underlying additive groups of the free polynomial algebra $(d=1)$ and of the free exterior algebra $(d=0)$, is in fact a part of the well-known boson-fermion correspondence.

For general $d$, the Hilbert-Poincaré series $P_{d}=P_{d}\left(z, q^{1 / 2}\right)$ of bigraded algebra $\mathcal{H}$ twisted by the $\operatorname{sign}(-1)^{\text {parity }}$ is the generalized $q$-exponential function:

$$
\sum_{n \geqslant 0, m \in \mathbb{Z}}(-1)^{m} \operatorname{dim}\left(\mathcal{H}_{n, m}\right) z^{n} q^{m / 2}=\sum_{n \geqslant 0} \frac{\left(-q^{1 / 2}\right)^{(1-d) n^{2}}}{(1-q) \ldots\left(1-q^{n}\right)} z^{n} \in \mathbb{Z}\left(\left(q^{1 / 2}\right)\right)[[z]] .
$$

In cases $d=0$ and $d=1$, this series decomposes in an infinite product:

$$
P_{0}=\left(q^{1 / 2} z ; q\right)_{\infty}=\prod_{i \geqslant 0}\left(1-q^{i+1 / 2} z\right), \quad P_{1}=\frac{1}{(z ; q)_{\infty}}=\prod_{i \geqslant 0} \frac{1}{1-q^{i} z}
$$

where we use the standard notation for the $q$-Pochhammer symbol:

$$
(x ; q)_{\infty}:=(1-x)(1-q x)\left(1-q^{2} x\right) \ldots
$$


Our general results in Section 6 will imply the following numerical result.

Theorem 2.3. For any $d \geqslant 0$, there exist integers $\delta^{(d)}(n, m)$ for all $n \geqslant 1$ and $m \in(d-1) n+2 \mathbb{Z}=(1-d) n^{2}+2 \mathbb{Z}$, such that for a given number $n$ we have $\delta(n, m) \neq 0$ only for 'finitely many' values of $m$, and

$$
P_{d}=\prod_{n \geqslant 1} \prod_{m \in \mathbb{Z}}\left(q^{m / 2} z^{n} ; q\right)_{\infty}^{\delta^{(d)}(n, m)} .
$$

The above implies the following decomposition:

$$
\frac{P_{d}\left(z, q^{1 / 2}\right)}{P_{d}\left(q z, q^{1 / 2}\right)}=\prod_{n \geqslant 1} \prod_{m \in \mathbb{Z}} \prod_{i=0}^{n-1}\left(1-q^{m / 2+i} z^{n}\right)^{\delta^{(d)}(n, m)} .
$$

Therefore, the limit

$$
P_{d}^{c l}(z):=\lim _{q^{1 / 2} \rightarrow 1} \frac{P_{d}\left(z, q^{1 / 2}\right)}{P_{d}\left(q z, q^{1 / 2}\right)} \in 1+z \mathbb{Z}[[z]]
$$

exists and has the form

$$
P_{d}^{c l}=\prod_{n \geqslant 1}\left(1-z^{n}\right)^{n c^{(d)}(n)}, \quad c^{(d)}(n) \in \mathbb{Z}
$$

where $c^{(d)}(n)=\sum_{m} \delta^{(d)}(n, m)$. It follows from the result of Reineke (see also Section 5.6) that $P_{d}^{c l}$ is an algebraic series satisfying

$$
P_{d}^{c l}(z)=1+(-1)^{(d-1)} z\left(P_{d}^{c l}(z)\right)^{d} .
$$

\subsection{Symmetric case}

In the case when matrix $\left(a_{i j}\right)_{i, j \in I}$ is symmetric one can repeat essentially all the considerations made for quivers with one vertex from Section 2.5.

First of all, the algebra $\mathcal{H}$ can be endowed with $\mathbb{Z}_{\geqslant 0}^{I} \times \mathbb{Z}$-grading:

$$
\mathcal{H}=\oplus_{\gamma, k} \mathcal{H}_{\gamma, k}, \quad \mathcal{H}_{\gamma, k}:=H^{k-\chi_{Q}(\gamma, \gamma)}\left(\mathrm{BG}_{\gamma}\right),
$$

where we recall $\chi_{Q}\left(\gamma_{1}, \gamma_{2}\right):=\sum_{i \in I} \gamma_{1}^{i} \gamma_{2}^{i}-\sum_{i, j \in I} a_{i j} \gamma_{1}^{i} \gamma_{2}^{j}$. The explicit formula for the product implies that

$$
a_{\gamma, k} \cdot a_{\gamma^{\prime}, k^{\prime}}=(-1)^{\chi_{Q}\left(\gamma, \gamma^{\prime}\right)} a_{\gamma^{\prime}, k^{\prime}} \cdot a_{\gamma, k} .
$$


This identity does not mean that $\mathcal{H}$ is supercommutative. We will modify the product on $\mathcal{H}$ in certain way, making it supercommutative.

Define a group homomorphism $\epsilon: \mathbb{Z}^{I} \rightarrow \mathbb{Z} / 2 \mathbb{Z}$ by the formula

$$
\epsilon(\gamma):=\chi_{Q}(\gamma, \gamma)(\bmod 2)
$$

The bilinear form

$$
\mathbb{Z}^{I} \otimes \mathbb{Z}^{I} \rightarrow \mathbb{Z} / 2 \mathbb{Z}, \quad \gamma_{1} \otimes \gamma_{2} \mapsto\left(\chi_{Q}\left(\gamma_{1}, \gamma_{2}\right)+\epsilon\left(\gamma_{1}\right) \epsilon\left(\gamma_{2}\right)\right)(\bmod 2)
$$

induces a symmetric bilinear form $\beta$ on $\mathbb{Z} / 2 \mathbb{Z}$-vector space $(\mathbb{Z} / 2 \mathbb{Z})^{I}$ which gives zero quadratic form.

It is easy to see that there exists a bilinear form $\psi$ on $(\mathbb{Z} / 2 \mathbb{Z})^{I}$ such that

$$
\psi\left(\gamma_{1}, \gamma_{2}\right)+\psi\left(\gamma_{2}, \gamma_{1}\right)=\beta\left(\gamma_{1}, \gamma_{2}\right)
$$

For example, choose an order on $I$ and define $\psi$ on elements $\left(e_{i}\right)_{i \in I}$ of the standard basis of $(\mathbb{Z} / 2 \mathbb{Z})^{I}$ by

$$
\psi\left(e_{i}, e_{j}\right)=\beta\left(e_{i}, e_{j}\right) \text { if } i>j, \quad \psi\left(e_{i}, e_{j}\right)=0 \text { if } i \leqslant j .
$$

We define a modified product on $\mathcal{H}$ by

$$
a_{1} \star a_{2}:=(-1)^{\psi\left(\gamma_{1}, \gamma_{2}\right)} a_{1} \cdot a_{2}, \quad a_{1} \in \mathcal{H}_{\gamma_{1}}, a_{2} \in \mathcal{H}_{\gamma_{2}} .
$$

The conditions on $\psi$ ensure that the new product is again associative, and $(\mathcal{H}, \star)$ is $\mathbb{Z}_{\geqslant 0}^{I} \times \mathbb{Z}$-graded supercommutative algebra, with the parity of the component $\mathcal{H}_{\gamma, k}$ given by $\epsilon(\gamma)$. Equivalently, one can use the parity given by $k(\bmod 2)$. It does not change the super structure on $\mathcal{H}$ because all nontrivial cohomology groups of $\mathrm{BG}_{\gamma}$ are concentrated in even degrees, hence

$$
k=\chi_{Q}(\gamma, \gamma)=\epsilon(\gamma)(\bmod 2)
$$

One can see easily that different choices of bilinear form $\psi$ lead to canonically isomorphic graded supercommutative algebras.

Here we formulate a general conjecture ${ }^{3}$.

Conjecture 2.1. In the symmetric case the $\mathbb{Z}_{\geqslant 0}^{I} \times \mathbb{Z}$-graded algebra $\mathcal{H} \otimes \mathbb{Q}$ is a free supercommutative algebra generated by a graded vector space $V$ over

\footnotetext{
${ }^{3}$ After the initial version of this text was posted on arXiv, A. Efimov gave a proof of our conjecture in [14]).
} 
$\mathbb{Q}$ of the form $V=V^{\text {prim }} \otimes \mathbb{Q}[x]$, where $x$ is an even variable of bidegree $(0,2) \in \mathbb{Z}_{\geqslant 0}^{I} \times \mathbb{Z}$, and for any given $\gamma$ the space $V_{\gamma, k}^{\text {prim }} \neq 0$ is non-zero (and finite-dimensional) only for finitely many $k \in \mathbb{Z}$.

In the special case of quiver with one vertex (see Section 2.5) this conjecture implies that all integers $\delta^{(d)}(n, m)$ in the decomposition in Theorem 2.3 in Section 2.5 have definite signs:

$$
(-1)^{m-1} \delta^{(d)}(n, m) \geqslant 0 .
$$

One evidence for the above conjecture is the admissibility property of the generating series from Sections 6.1 and 6.2 (see e.g. Theorem 6.2 and Corollary 6.2 below). Those series generalize the product from Theorem 2.3.

\subsection{Grading by the Heisenberg group and twisted graded algebras}

In general, for not necessarily symmetric matrix $\left(a_{i j}\right)_{i, j \in I}$, the interaction of the product in $\mathcal{H}$ with the cohomological grading can be treated in the following way. Let $\Gamma$ be an abelian group and $B: \Gamma \otimes \Gamma \rightarrow \mathbb{Z}$ be a bilinear form (in our case $\Gamma=\mathbb{Z}^{I}$ is the group of dimension vectors and $B=\chi_{Q}$ ). We associate with $(\Gamma, B)$ the discrete Heisenberg group Heis ${ }_{\Gamma, B}$ which is the set $\Gamma \times \mathbb{Z}$ endowed with the multiplication

$$
\left(\gamma_{1}, k_{1}\right) \cdot\left(\gamma_{2}, k_{2}\right):=\left(\gamma_{1}+\gamma_{2}, k_{1}+k_{2}-2 B\left(\gamma_{1}, \gamma_{2}\right)\right)
$$

Our Cohomological Hall algebra $\mathcal{H}$ can be graded by the non-commutative group Heis $\Gamma_{\Gamma, B}$ :

$$
\mathcal{H}=\oplus_{\gamma, k} \mathcal{H}_{(\gamma, k)}, \quad \mathcal{H}_{(\gamma, k)}=H^{k}\left(\mathrm{BG}_{\gamma}\right)
$$

This can be generalized such as follows. Let $(\mathcal{T}, \otimes)$ be a symmetric monoidal category endowed with an even invertible object $\mathbb{T}_{\mathcal{T}}$, i.e. such an invertible object that the commutativity morphism $\mathbb{T}_{\mathcal{T}} \otimes \mathbb{T}_{\mathcal{T}} \rightarrow \mathbb{T}_{\mathcal{T}} \otimes \mathbb{T}_{\mathcal{T}}$ is the identity morphism. Let us fix $(\Gamma, B)$ as above. We define a twisted graded monoid $^{4}$ in $\mathcal{T}$ as a collection of objects $\left(\mathcal{H}_{\gamma}\right)_{\gamma \in \Gamma}$ together with a collection

\footnotetext{
${ }^{4}$ We use the word "algebra" instead of "monoid" for additive monoidal categories.
} 
of morphisms

$$
m_{\gamma_{1}, \gamma_{2}}: \mathcal{H}_{\gamma_{1}} \otimes \mathcal{H}_{\gamma_{2}} \rightarrow \mathbb{T}_{\mathcal{T}}^{\otimes B\left(\gamma_{1}, \gamma_{2}\right)} \otimes \mathcal{H}_{\gamma_{1}+\gamma_{2}}
$$

and a unit morphism $1_{\mathcal{C}} \rightarrow \mathcal{H}_{0}$, satisfying an obvious extension of the usual associativity and the unity axioms. In our situation $(\mathcal{T}, \otimes)$ is the tensor category of $\mathbb{Z}$-graded abelian groups (with the usual Koszul sign rule for the commutativity morphism), and $\mathbb{T}_{\mathcal{T}}=\mathbb{Z}[-2]$ is the group $\mathbb{Z}$ placed in degree +2 , i.e., the cohomology of the pair $H^{\bullet}\left(\mathbb{C} P^{1}, p t\right)$. Object $\mathcal{H}_{\gamma}$ is just $H^{\bullet}\left(\mathrm{M}_{\gamma}^{\text {univ }}\right)=H^{\bullet}\left(\mathrm{BG}_{\gamma}\right)$.

Let us assume additionally that the form $B$ is symmetric, and we are given an invertible object $\mathbb{T}_{\mathcal{T}}^{\otimes 1 / 2} \in \mathcal{T}$ such that $\left(\mathbb{T}_{\mathcal{T}}^{\otimes 1 / 2}\right)^{\otimes 2} \simeq \mathbb{T}_{\mathcal{T}}$. Then for any twisted graded monoid $\mathcal{H}=\left(\mathcal{H}_{\gamma}\right)_{\gamma \in \Gamma}$ we define the modified graded monoid by

$$
\mathcal{H}_{\gamma}^{\bmod }:=\mathcal{H}_{\gamma} \otimes\left(\mathbb{T}_{\mathcal{T}}^{\otimes 1 / 2}\right)^{\otimes B(\gamma, \gamma)}
$$

which is an ordinary (untwisted) $\Gamma$-graded monoid in $\mathcal{T}$. In particular, for the category of $\mathbb{Z}$-graded abelian groups we take $\mathbb{T}_{\mathcal{T}}^{\otimes 1 / 2}$ to be $\mathbb{Z}$ in degree +1 . In this way, we obtain another description of the modified product $\star$ from the previous section. Notice that in this case object $\mathbb{T}_{\mathcal{T}}^{\otimes 1 / 2}$ is not even.

\subsection{Non-symmetric example: quiver $\boldsymbol{A}_{2}$}

The quiver $A_{2}$ has two vertices $\{1,2\}$ and one arrow $1 \leftarrow 2$. The Cohomological Hall algebra $\mathcal{H}$ contains two subalgebras $\mathcal{H}_{L}, \mathcal{H}_{R}$ corresponding to representations supported at the vertices 1 and 2, respectively. Clearly each subalgebra $\mathcal{H}_{L}, \mathcal{H}_{R}$ is isomorphic to the Cohomological Hall algebra for the quiver $A_{1}=Q_{0}$. Hence, it is an infinitely generated exterior algebra (see Section 2.5). Let us denote the generators by $\xi_{i}, i=0,1, \ldots$, for the vertex 1 and by $\eta_{i}, i=0,1, \ldots$, for the vertex 2 . Each generator $\xi_{i}$ or $\eta_{i}$ corresponds to an additive generator of the group $H^{2 i}(\operatorname{BGL}(1, \mathbb{C})) \simeq \mathbb{Z} \cdot x^{i}$. Then one can check that $\xi_{i}, \eta_{j}, i, j \geqslant 0$ satisfy the relations

$$
\xi_{i} \xi_{j}+\xi_{j} \xi_{i}=\eta_{i} \eta_{j}+\eta_{j} \eta_{i}=0, \quad \eta_{i} \xi_{j}=\xi_{j+1} \eta_{i}-\xi_{j} \eta_{i+1}
$$

Let us introduce the elements $\nu_{i}^{1}=\xi_{0} \eta_{i}, i \geqslant 0$ and $\nu_{i}^{2}=\xi_{i} \eta_{0}, i \geqslant 0$. It is easy to see that $\nu_{i}^{1} \nu_{j}^{1}+\nu_{j}^{1} \nu_{i}^{1}=0$, and similarly the generators $\nu_{i}^{2}$ anticommute. Thus we have two infinite Grassmann subalgebras in $\mathcal{H}$ corresponding to these two choices: $\mathcal{H}^{(1)} \simeq \bigwedge\left(\nu_{i}^{1}\right)_{i \geqslant 0}$ and $\mathcal{H}^{(2)} \simeq \bigwedge\left(\nu_{i}^{2}\right)_{i \geqslant 0}$. One can directly check the following result. 
Proposition 2.1. The multiplication (from the left to the right) induces isomorphisms of graded abelian groups

$$
\mathcal{H}_{L} \otimes \mathcal{H}_{R} \stackrel{\sim}{\longrightarrow} \mathcal{H}, \quad \mathcal{H}_{R} \otimes \mathcal{H}^{(i)} \otimes \mathcal{H}_{L} \stackrel{\sim}{\longrightarrow} \mathcal{H}, \quad i=1,2
$$

In other words, we obtain a canonical isomorphism (depending on $i=$ 1,2 ) of graded abelian groups

$$
\begin{aligned}
& H^{\bullet}(\operatorname{BGL}(n, \mathbb{C}) \times \operatorname{BGL}(m, \mathbb{C})) \\
& \simeq \bigoplus_{0 \leqslant k \leqslant \min (n, m)} H^{\bullet}(\operatorname{BGL}(m-k, \mathbb{C}) \times \operatorname{BGL}(k, \mathbb{C}) \\
& \quad \times \operatorname{BGL}(n-k, \mathbb{C}))[-2(n-k)(m-k)]
\end{aligned}
$$

Passing to generating series we obtain the standard identity

$$
\left(q^{1 / 2} \widehat{\mathbf{e}}_{1} ; q\right)_{\infty} \cdot\left(q^{1 / 2} \widehat{\mathbf{e}}_{2} ; q\right)_{\infty}=\left(q^{1 / 2} \widehat{\mathbf{e}}_{2} ; q\right)_{\infty} \cdot\left(q^{1 / 2} \widehat{\mathbf{e}}_{12} ; q\right)_{\infty} \cdot\left(q^{1 / 2} \widehat{\mathbf{e}}_{1} ; q\right)_{\infty},
$$

where non-commuting variables $\widehat{\mathbf{e}}_{1}, \widehat{\mathbf{e}}_{2}, \widehat{\mathbf{e}}_{12}$ satisfy relations of the Heisenberg group (with $-q^{1 / 2}$ corresponding to the central element):

$$
\widehat{\mathbf{e}}_{1} \cdot \widehat{\mathbf{e}}_{2}=q^{-1} \widehat{\mathbf{e}}_{2} \cdot \widehat{\mathbf{e}}_{1}=-q^{-1 / 2} \widehat{\mathbf{e}}_{12} .
$$

The same example of quiver $A_{2}$ was studied in [33], Section 6.4. The generating series $\mathbf{E}\left(q^{1 / 2}, x\right)$ used there is related with the $q$-Pochhammer symbol by the following change of variables:

$$
\mathbf{E}\left(-q^{1 / 2}, x\right)=\left(q^{1 / 2} x ; q\right)_{\infty}
$$

where both sides are viwed as formal series in $x$ with coefficients in the field $\mathbb{Q}\left(q^{1 / 2}\right)$ of rational functions in variables $q^{1 / 2}$.

\subsection{Questions about other algebraic structures on $\mathcal{H}$}

One may wonder whether $\mathcal{H}$ carries other natural algebraic structures. For example, it would be nice to have a structure of Hopf algebra on $\mathcal{H}$, as a step towards Conjecture 2.1 from Section 2.6, and also by analogy with quantum enveloping algebras. 
One can see immediately that $\mathcal{H}$ carries a structure of (super) cocommutative coassociative coalgebra with the coproduct

$$
\mathcal{H}_{\gamma_{1}+\gamma_{2}} \rightarrow \mathcal{H}_{\gamma_{1}} \otimes \mathcal{H}_{\gamma_{2}}
$$

induced by the operation of the direct sum on representations:

$$
\mathrm{M}_{\gamma_{1}}^{\mathrm{univ}} \times \mathrm{M}_{\gamma_{2}}^{\mathrm{univ}} \rightarrow \mathrm{M}_{\gamma_{1}+\gamma_{2}}^{\mathrm{univ}}
$$

which is realized geometrically via any embedding $\mathbb{C}^{\infty} \oplus \mathbb{C}^{\infty} \hookrightarrow \mathbb{C}^{\infty}$. Unfortunately, this coproduct is not compatible with the product on $\mathcal{H}$. In Section 6.5, we will define a supercommutative associative product on $\mathcal{H}$, which is also related to the operation of the direct sum, and used in the study of motivic DT-series of $\mathcal{H}$. We do not know any reasonable relation between this supercommutative product and the product introduced in Section 2.2.

Another potentially interesting structure is associated with the operation of taking multiple copies of an object. This gives a morphism of cohomology

$$
\mathcal{H}_{n \gamma} \rightarrow \mathcal{H}_{\gamma} \otimes H^{\bullet}(\operatorname{BGL}(n, \mathbb{C}))
$$

for any $n \geqslant 0$. It comes from the map

$$
\mathrm{M}_{\gamma}^{\text {univ }} \times \operatorname{BGL}(n, \mathbb{C}) \rightarrow \mathrm{M}_{n \gamma}^{\text {univ }}
$$

and is realized geometrically via any embedding $\mathbb{C}^{\infty} \otimes \mathbb{C}^{n} \hookrightarrow \mathbb{C}^{\infty}$.

\section{Generalization of COHA for smooth algebras}

Up to now, homotopy types of the moduli stacks of representations were all very simple, namely, just products of classifying spaces of groups $\operatorname{GL}(n, \mathbb{C})$, $n \geqslant 0$. In this section, we are going to discuss several generalizations of the Cohomological Hall algebra, in particular for representations of smooth algebras. Also, we will use not only the usual cohomology with coefficients in $\mathbb{Z}$, but other cohomology groups of algebraic varieties, like, e.g., de Rham or étale cohomology.

\subsection{Cohomology theories}

Here we collect preliminary material about cohomology theories for algebraic varieties and fix some notations. 
Definition 3.1. Let $\mathbf{k}$ be a field, and $K$ be another field, $\operatorname{char}(K)=$ 0 . Assume that we are given a $K$-linear Tannakian category $\mathcal{C}$. A precohomology theory over $\mathbf{k}$ with values in $\mathcal{C}$ is a contravariant tensor functor $\mathbf{H}^{\bullet}$ from the category of schemes of finite type over $\mathbf{k}$ to the tensor category $\mathcal{C}^{\mathbb{Z}-\text { gr }}$ of $\mathbb{Z}$-graded objects in $\mathcal{C}$ endowed with Koszul rule of signs.

Here is the list of standard examples of (pre-)cohomology theories, $\mathcal{C}=$ $K$ - mod:

- $($ case $\operatorname{char} \mathbf{k}=0, \mathbf{k} \subset \mathbb{C})$ : rational Betti cohomology, $K=\mathbb{Q}$,

- (case char $\mathbf{k}=0)$ : de Rham cohomology, $K=\mathbf{k}$,

- (case char $\mathbf{k} \neq l$ for prime $l$ ): étale cohomology, $K=\mathbb{Q}_{l}$.

In the case $\mathbf{k} \subset \mathbb{C}$ and Betti cohomology one can enhance $\mathcal{C}$ from $\mathbb{Q}$ - mod to the Tannakian category of polarizable mixed Hodge structures. Similarly, in the étale case we can take $\mathcal{C}$ to be the category of continuous $l$-adic representations of the absolute Galois group of $\mathbf{k}$.

Definition 3.2. A pre-cohomology theory $\mathbf{H}^{\bullet}$ is called cohomology theory if there exists a fiber functor $\mathcal{C} \rightarrow K^{\prime}-\bmod$ for some field extension $K^{\prime} \supset K$ such that the resulting pre-cohomology theory is obtained by the extension of scalars from one of the standard theories.

All the above examples give in fact cohomology theories. Notice that in our definition one can mention only étale cohomology, since in the case char $\mathbf{k}=0$ we have a chain of comparison isomorphisms

$$
\text { de } \mathrm{Rham} \simeq \text { Betti } \simeq \text { étale }
$$

In fact, in what follows we will need only a pre-cohomology theory satisfying the usual properties of cohomology, and the definition above is given in order to avoid a long discussion of cohomology theories. The optimal framework should be that of triangulated category of motives; see Section 3.5.

We denote $\mathbf{H}^{2}\left(\mathbb{P}_{\mathbf{k}}^{1}\right)$ by $K(-1)$ understood as an element of $\mathcal{C}$, and set

$$
\mathbb{T}=\mathbb{T}_{\mathbf{H}} \cdot:=K(-1)[-2] \in \mathcal{C}^{\mathbb{Z}-g r} .
$$

\subsection{Smooth algebras}

Here we recall the notion of a smooth algebra from [32]. 
Definition 3.3. An associative unital algebra $R$ over a field $\mathbf{k}$ is called smooth if it is finitely generated and formally smooth in the sense of D. Quillen and J. Cuntz, i.e., if the bimodule $\Omega_{R}^{1}:=\operatorname{Ker}\left(R \otimes_{\mathbf{k}} R \stackrel{\text { mult }}{\longrightarrow} R\right)$ is projective. Here mult : $R \otimes_{\mathbf{k}} R \rightarrow R$ is the product.

For a finitely presented algebra $R$ it is easy to check smoothness using a finite amount of calculations; see [10] or [32], Section 1.1.3. The property of formal smoothness is equivalent to the lifting property for non-commutative nilpotent extensions: for any associative unital algebra $A$ over $\mathbf{k}$ and a nilpotent two-sided ideal $J \subset A, J^{n}=0$ for some $n>0$, and any homomorphism $\phi: R \rightarrow A / J$ there exists a lifting of $\phi$ to a homomorphism $R \rightarrow A$.

Basic examples of smooth algebras are matrix algebras, path algebras of finite quivers and algebras of functions on smooth affine curves.

The notion of a smooth algebra is invariant under Morita equivalence, and closed under free products and finite localizations. The latter means that we can add two-sided inverses to a finite collection of elements in $R$ (or, more generally, to a finite collection of rectangular matrices with coefficients in $R$ ). For example, the group ring of a free finitely generated group

$$
\mathbf{k}\left[\text { Free }_{d}\right]=\mathbf{k}\left\langle x_{1}^{ \pm 1}, \ldots, x_{d}^{ \pm 1}\right\rangle
$$

is a smooth algebra.

For a smooth algebra $R$ and for any finite-dimensional algebra $T$ over $\mathbf{k}$ (e.g. $T=\operatorname{Mat}(n \times n, \mathbf{k}))$, the scheme of homomorphisms $R \rightarrow T$ is a smooth affine scheme. Indeed, this is a closed subscheme of an affine space $\mathbb{A}_{\mathbf{k}}^{g_{R} \operatorname{dim} T}$ where $g_{R}$ is number of generators of $R$, hence it is an affine scheme of finite type over k. Also this scheme enjoys the lifting property for (commutative) nilpotent extensions (as follows from formal smoothness), hence it is smooth.

If $R$ is smooth then the abelian category of $R$-modules is hereditary, i.e., $\operatorname{Ext}^{i}(E, F)=0$ for for any two objects $E, F$ and any $i \geqslant 2$. If $E, F$ are finite-dimensional over $\mathbf{k}$ then $\operatorname{spaces} \operatorname{Hom}(E, F), \operatorname{Ext}^{1}(E, F)$ are also finitedimensional.

\subsection{Cohomological Hall algebra for an $I$-bigraded smooth algebra}

For a finite set $I$, we call an unital associative algebra $R / \mathbf{k}$ I-bigraded if $R$ is decomposed (as a vector space) into the direct sum $R=\oplus_{i, j \in I} R_{i j}$ in such a way that $R_{i j} \cdot R_{j k} \subset R_{i k}$. Equivalently, $R$ is $I$-bigraded if we are given a morphism of unital algebras $\mathbf{k}^{I} \rightarrow R$. 
Let now $R$ be an $I$-bigraded smooth algebra. It follows that any finitedimensional representation $E$ of $R$ decomposes into a direct sum of finitedimensional vector spaces $E_{i}, i \in I$.

For any dimension vector $\gamma=\left(\gamma^{i}\right)_{i \in I} \in \mathbb{Z}_{\geqslant 0}^{I}$ the scheme $\mathrm{M}_{\gamma}=\mathrm{M}_{\gamma}^{R}$ of representations of $R$ in coordinate spaces $E_{i}=\mathbf{k}^{\gamma^{i}}, i \in I$ is a smooth affine scheme. Any choice of a finite set of $I$-bigraded generators of $R$ gives a closed embedding of $\mathrm{M}_{\gamma}$ into the affine space $\mathrm{M}_{\gamma}^{Q}$ for some quiver $Q$ with the set of vertices equal to $I$.

Let us make the following assumption:

We are given a bilinear form $\chi_{R}: \mathbb{Z}^{I} \otimes \mathbb{Z}^{I} \rightarrow \mathbb{Z}$ such that for any two dimension vectors $\gamma_{1}, \gamma_{2} \in \mathbb{Z}_{\geqslant 0}^{I}$ and for any two representations $E_{i} \in \mathrm{M}_{\gamma_{i}}(\overline{\boldsymbol{k}})$ we have the equality

$$
\operatorname{dim} \operatorname{Hom}\left(E_{1}, E_{2}\right)-\operatorname{dim} \operatorname{Ext}^{1}\left(E_{1}, E_{2}\right)=\chi_{R}\left(\gamma_{1}, \gamma_{2}\right)
$$

Here $\overline{\mathbf{k}}$ is an algebraic closure of $\mathbf{k}$, and $E_{1}, E_{2}$ are considered as representations of algebra $R \otimes_{\mathbf{k}} \overline{\mathbf{k}}$ over $\overline{\mathbf{k}}$. The assumption implies that the smooth scheme $\mathrm{M}_{\gamma}$ is equidimensional for any given $\gamma$ and

$$
\operatorname{dim} \mathrm{M}_{\gamma}=-\chi_{R}(\gamma, \gamma)+\sum_{i}\left(\gamma^{i}\right)^{2}
$$

For example, if the algebra $R$ is obtained from the path algebra of a finite quiver $Q$ by a finite number of localizations, then $\chi_{R}=\chi_{Q}$ and the scheme $\mathrm{M}_{\gamma}$ is a Zariski open subset of the affine space $\mathrm{M}_{\gamma}^{Q} \simeq \mathbb{A}_{\mathbf{k}}^{\sum_{i j} a_{i j} \gamma^{i} \gamma^{j}}$.

Now we can use any cohomology theory $\mathbf{H}^{\bullet}$ and obtain the corresponding Cohomological Hall algebra, which is a twisted associative algebra (in the sense of Section 2.7) in tensor category $\mathcal{C}^{\mathbb{Z}-g r}$ with

$$
\mathbb{T}_{\mathcal{T}}:=\mathbb{T}=K(-1)[-2]
$$

The main point is that spaces $\mathrm{M}_{\gamma_{1}, \gamma_{2}}$ of representations of $R$ in block uppertriangular matrices, are smooth closed subvarieties of $\mathrm{M}_{\gamma_{1}+\gamma_{2}}$. The definition of the product and the proof of associativity given in Sections 2.2 and 2.3 work without modifications in the general case of smooth algebras and cohomology theories. In this way we obtain COHA $\mathcal{H}:=\mathcal{H}_{R}$ for a smooth $I$-bigraded algebra $R$ which satisfies the above assumption. 


\subsection{Square root of Tate motive and modified product}

Let $\mathcal{T}$ be a rigid tensor category over field $K$, and $E$ be an even invertible object of $\mathcal{T}$. Then one can formally add to $\mathcal{T}$ an even tensor square root $E^{\otimes 1 / 2}$ (see Section 2.7). Moreover, if $\mathcal{T}$ is Tannakian, then the new tensor category $\mathcal{T}\left(E^{\otimes 1 / 2}\right)$ is also Tannakian. This can be explained explicitly as follows. Let us assume that we are given a fiber functor $\mathcal{T} \rightarrow K-\bmod$, i.e., identify $\mathcal{T}$ with the tensor category of finite-dimensional representations of a pro-affine algebraic group $G$ over $K$. Object $E$ gives a homomorphism $t: G \rightarrow \mathbb{G}_{m, K} \simeq \operatorname{Aut}(E)$. Denote by $G^{(2)}$ the fibered product

$$
G^{(2)}:=\lim _{\longleftarrow}\left(\begin{array}{rr} 
& \mathbb{G}_{m} \\
& \lambda \mapsto \lambda^{2} \downarrow \\
G \stackrel{t}{\longrightarrow} & \mathbb{G}_{m}
\end{array}\right) .
$$

Then $\mathcal{T}\left(E^{\otimes 1 / 2}\right)$ is canonically equivalent to the tensor category of representations of $G^{(2)}$. It is easy to see that the ring $K_{0}\left(G^{(2)}-\operatorname{Rep}\right)$ is canonically isomorphic to $K_{0}(G-\operatorname{Rep})[x] /\left(x^{2}-[E]\right)$, where $x \mapsto\left[E^{\otimes 1 / 2}\right]$.

One can apply these considerations to the Tannakian category $\mathcal{C}$ associated with a cohomology theory $\mathbf{H}^{\bullet}$. We take $E:=K(-1)$. The square root $E^{\otimes 1 / 2}$ can be denoted by $K(-1 / 2)$. Usually, there is no square root of $K(-1)$ in $\mathcal{C}$. For classical cohomology it exists only when $\mathbf{k}$ has positive characteristic and a square root is one of summands of $\mathbf{H}^{1}(C)$ where $C$ is a supersingular elliptic curve. In Section 4, we will develop a generalization of cohomology for varieties with potential. In this more general framework a square root of $K(-1)$ exists much more often, e.g., it is enough to assume that $\mathbf{k}$ contains a $\sqrt{-1}$. Anyhow, in what follows we will assume that the Tannakian category $\mathcal{C}$ for the cohomological theory contains a chosen square root $K(-1 / 2)$ (if it cannot be found in the original category, we will add it formally as explained above). We will also denote

$$
\mathbb{T}^{\otimes 1 / 2}:=K(-1 / 2)[-1] \in \mathcal{C}^{\mathbb{Z}-g r}
$$

Let $\mathcal{H}=\oplus_{\gamma \in \mathbb{Z}_{\geqslant 0}^{I}} \mathcal{H}_{\gamma}$ be the Cohomological Hall algebra of an $I$-bigraded smooth algebra $R$.

Definition 3.4. The modified Cohomological Hall algebra $\mathcal{H}^{\text {mod }}$ is given by

$$
\mathcal{H}^{\bmod }:=\oplus_{\gamma \in \mathbb{Z}_{\geqslant 0}^{I}} \mathcal{H}_{\gamma}^{\bmod }, \quad \mathcal{H}_{\gamma}^{\bmod }:=\mathcal{H}_{\gamma} \otimes\left(\mathbb{T}^{\otimes 1 / 2}\right)^{\otimes \chi_{R}(\gamma, \gamma)}
$$


For any sublattice $\Gamma \subset \mathbb{Z}^{I}$ such that the restriction of $\chi_{R}$ to $\Gamma$ is symmetric, the restricted modified algebra

$$
\bigoplus_{\gamma \in \Gamma \cap \mathbb{Z}_{\geqslant 0}^{I}} \mathcal{H}_{\gamma}^{\bmod }
$$

is an associative $\Gamma$-graded algebra in tensor category $\mathcal{C}^{\mathbb{Z}-g r}$.

\subsection{Algebra in the triangulated category of motives}

We can try to use a more refined object instead of graded cohomology spaces (for some classical cohomology theory). A natural candidate is the motive of the ind-schemes $\mathrm{M}_{\gamma}^{\text {univ }}$ understood as an element of Voevodsky triangulated category of motives (see [54]). More precisely, we should maybe use a dg enhancement constructed in [9] and in [5]. We expect that the Cohomological Hall algebra has a natural enhancement to an $A_{\infty}$-algebra in the tensor $\mathrm{dg}$ category of mixed motives, with higher multiplications somehow governed by manifolds of flags of representations. Those are the spaces $\mathrm{M}_{\gamma_{1}, \ldots, \gamma_{n}}$ from the proof of Theorem 5.1 in Section 5.2 below. The invertible object $\mathbb{T}$ used in the definition of a twisted associative algebra (see Section 2.7) is the graded space $\mathbb{Z}(-1)[-2]$, the Tate motive of the cohomology of pair $\left(\mathbb{P}^{1}, p t\right)$.

\subsection{Equivariant parameters in arrows and families of quivers}

Let us consider for simplicity the case of the path algebra of a quiver. One can introduce a torus action on the path algebra by rescaling independently individual arrows. Then the representation varieties will be endowed with the action of an additional torus $\mathbf{T}$, and one can use the $\mathbf{T}$-equivariant cohomology. In this way, we define COHA as $H^{\bullet}(B \mathbf{T})$-module. This is a particular case of a more general construction, when one has a family over some base $\mathcal{B}$ of smooth $I$-bigraded algebras. The case of $\mathbf{T}$-action corresponds to $\mathcal{B}:=B \mathbf{T}$ (the classifying space of $\mathbf{T})$.

\subsection{Complex cobordisms}

The natural framework for the theory of virtual fundamental classes in the case of almost complex manifolds and complex obstruction bundles is not the usual cohomology theory but the complex cobordism theory. For an element $(f: Y \rightarrow X) \in \Omega_{n}(X)$ of the $n$th bordism group of $X$, where $Y$ is a 
compact manifold with stable complex structures, and a homogeneous monomial of characteristic classes $\alpha=c_{1}\left(T_{Y}\right)^{a_{1}} \ldots c_{k}\left(T_{Y}\right)^{a_{k}}$, one can define the corresponding element $f_{*}[P . D .(\alpha)] \in H_{\bullet}(X)$. Here P.D.( $\left.\alpha\right)$ is the Poincaré dual homology class to $\alpha$. This means that after tensoring by $\mathbb{Q}$ we replace the cohomology by the tensor product of cohomology with $H^{\bullet}(B U(\infty))$ (the graded version of the algebra of symmetric polynomials). In order to pass back to complex bordisms groups we should keep track of the action of characteristic classes of the virtual tangent bundles of the Grassmannians and of the moduli stacks on their virtual fundamental classes. Therefore, if we define the Cohomological Hall algebra using complex bordisms instead of cohomology groups, we obtain $H^{\bullet}(B U(\infty)$, Q)-linear product on $H^{\bullet}(B U(\infty), \mathbb{Q}) \otimes \mathcal{H}$. It can be considered as a deformation of the original COHA with the base $\operatorname{Spec} H^{\bullet}(B U(\infty), \mathbb{Q}) \simeq \mathbb{A}_{\mathbb{Q}}^{\infty}$.

\section{Exponential Hodge structures and COHA for a smooth algebra with potential}

\subsection{Short synopsis}

Definition 4.1. For a complex algebraic variety $X$ and a function $f \in$ $\mathcal{O}(X)$ regarded as a regular map $f: X \rightarrow \mathbb{C}$, we define the rapid decay cohomology $H^{\bullet}(X, f)$ as the limit for real $t \rightarrow-\infty$ of the cohomology of the pair $H^{\bullet}\left(X, f^{-1}\left(S_{t}\right)\right)$, where

$$
S_{t}:=\{z \in \mathbb{C} \mid \operatorname{Re} z<t\}
$$

The cohomology stabilizes at some $t_{0} \in \mathbb{R}, t_{0} \ll 0$ (also in the definition one can replace $f^{-1}\left(S_{t}\right)$ by $\left.f^{-1}(t)\right)$. The origin of the term "rapid decay" will become clear later in Section 4.5.

The cohomology $H^{\bullet}(X, f)$ behaves similarly to the usual cohomology. In particular, for a map $\pi: Y \rightarrow X$ compatible with functions $f_{Y} \in \mathcal{O}(Y), f_{X} \in$ $\mathcal{O}(X)$ in the sense that $f_{Y}=\pi^{*} f_{X}$, we have the pullback $\pi^{*}: H^{\bullet}\left(X, f_{X}\right) \rightarrow$ $H^{\bullet}\left(Y, f_{Y}\right)$. If $\pi$ is proper and both $X$ and $Y$ are smooth, then we have the pushforward morphism $\pi_{*}: H^{\bullet}\left(Y, f_{Y}\right) \rightarrow H^{\bullet+2}\left(\operatorname{dim}_{\mathbb{C}}(X)-\operatorname{dim}_{\mathbb{C}}(Y)\right)\left(X, f_{X}\right)$.

Similarly to the usual cohomology, there is a multiplication (Künneth) morphism

$$
\otimes: H^{\bullet}\left(X, f_{X}\right) \otimes H^{\bullet}\left(Y, f_{Y}\right) \rightarrow H^{\bullet}\left(X \times Y, f_{X} \boxplus f_{Y}\right)
$$


where the Thom-Sebastiani sum $\boxplus$ is given by

$$
f_{X} \boxplus f_{Y}:=\operatorname{pr}_{X \times Y \rightarrow X}^{*} f_{X}+\operatorname{pr}_{X \times Y \rightarrow Y}^{*} f_{Y}
$$

For any smooth $I$-bigraded algebra $R$ as in Section 3.3 and any element $W \in R /[R, R]$ we obtain a function $W_{\gamma}$ on $\mathrm{M}_{\gamma}$ which is invariant under $\mathrm{G}_{\gamma}$-action. It is given by the trace of $W$ in a representation. Hence we can apply the formalism of Sections $2.1-2.3$ to rapid decay cohomology and define the Cohomological Hall algebra as

$$
\mathcal{H}=\oplus_{\gamma} \mathcal{H}_{\gamma}, \quad \mathcal{H}_{\gamma}:=H_{\mathrm{G}_{\gamma}}^{\bullet}\left(\mathrm{M}_{\gamma}, W_{\gamma}\right)
$$

In the case when $R$ is the path algebra of a quiver $Q$, the element $W$ can be considered as a linear combination of cyclic paths in $Q$. It can be thought of as a potential for a general multi-matrix model (see e.g. [16]).

In the next sections we will define an analog of mixed Hodge structure on $H^{\bullet}(X, f) \otimes \mathbb{Q}$, in particular an analog of the weight filtration. There is a topological formula for the weight filtration similar to the classical case.

\subsection{Exponential mixed Hodge structures}

The goal of this section is to define a cohomology theory for pairs $(X, f)$ consisting of a smooth algebraic variety $X$ over $\mathbb{C}$ and a function $f \in \mathcal{O}(X)$, with values in certain Tannakian $\mathbb{Q}$-linear category EMHS (see Definition 4.2 below) which we call the category of exponential mixed Hodge structures. Definition of the category EMHS will be based on the properties of two equivalent Tannakian categories $\mathfrak{A}_{0}$ and $\mathfrak{B}_{0}$ described below. The discussion below is essentially a reformulation of well-known results of N. Katz (see [29], Chapter 12) on the additive convolution of $\mathcal{D}$-modules and perverse sheaves on the affine line $\mathbb{A}_{\mathbb{C}}^{1}$.

First, consider the abelian category $\mathfrak{A}=\operatorname{Hol}_{r s}\left(\mathbb{A}_{\mathbb{C}}^{1}\right)$ of holonomic $\mathcal{D}$ modules with regular singularities on the standard affine line endowed with coordinate $x$. By Fourier transform FT this category is identified with the category $\mathfrak{B} \simeq \mathrm{FT}(\mathfrak{A})$ of holonomic $\mathcal{D}$-modules $M$ on the dual line (endowed with the canonical coordinate $y=-d / d x$ ) such that the direct image of $M$ to $\mathbb{C P}^{1}$ has a regular singularity at 0 , no singularities at $\mathbb{A}_{\mathbb{C}}^{1}-\{0\}$, and a possibly irregular singularity at $\infty$ of the exponential type. The latter condition means that after the base change to $\mathbb{C}\left(\left(y^{-1}\right)\right)$ we have

$$
M \otimes_{\mathbb{C}[y]} \mathbb{C}\left(\left(y^{-1}\right)\right) \simeq \bigoplus_{i} \mathfrak{e x p}\left(\lambda_{i} y\right) \otimes_{\mathbb{C}\left(\left(y^{-1}\right)\right)} M_{i}
$$


where $\lambda_{i} \in \mathbb{C}$ run through a finite set $I$, and $M_{i}$ are regular holonomic $\mathcal{D}$-modules on the formal punctured disc $\operatorname{Spf}\left(\mathbb{C}\left(\left(y^{-1}\right)\right)\right)$. Here $\mathfrak{e x p}\left(\lambda_{i} y\right)$ denotes the $\mathcal{D}$-module on $\operatorname{Spf}\left(\mathbb{C}\left(\left(y^{-1}\right)\right)\right)$ which is a free module over $\mathbb{C}\left(\left(y^{-1}\right)\right)$ with generator $e$ satisfying the same equation

$$
\left(d / d y-\lambda_{i}\right) \cdot e=0
$$

as the exponential function $\exp \left(\lambda_{i} y\right)$.

Let us denote by $\mathfrak{B}_{0}$ the abelian category of holonomic $\mathcal{D}$-modules on $\mathbb{A}_{\mathbb{C}}^{1}-\{0\}$ with no singularities at $\mathbb{A}_{\mathbb{C}}^{1}-\{0\}$, such that its direct image to $\mathbb{C P}^{1}$ has a regular singularity at 0 and an exponential type singularity at $\infty$. In particular, any object of $\mathfrak{B}_{0}$ is an algebraic vector bundle over $\mathbb{A}_{\mathbb{C}}^{1}-\{0\}=$ Spec $\mathbb{C}\left[y, y^{-1}\right]$ endowed with a connection. The inclusion

$$
j: \mathbb{A}_{\mathbb{C}}^{1}-\{0\} \hookrightarrow \mathbb{A}_{\mathbb{C}}^{1}
$$

gives two adjoint exact functors

$$
j^{*}: \mathfrak{B} \rightarrow \mathfrak{B}_{0}, \quad j_{*}: \mathfrak{B}_{0} \rightarrow \mathfrak{B}
$$

such that $j^{*} \circ j_{*}=\operatorname{id}_{\mathfrak{B}_{0}}$ (hence $\mathfrak{B}_{0}$ can be considered as a full abelian subcategory of $\mathfrak{B}$ ) and $\Pi:=j_{*} \circ j^{*}$ is an exact idempotent endofunctor of $\mathfrak{B}$, given by the tensor product $M \mapsto \mathbb{C}\left[y, y^{-1}\right] \otimes_{\mathbb{C}[y]} M$. A $\mathcal{D}$-module $M \in \mathfrak{B}$ belongs to $\mathfrak{B}_{0}$ if and only if the operator of multiplication by $y$ is invertible in $M$. In what follows, we will use the same notation $\Pi$ for exact idempotent endofunctors in several abelian categories closely related with the category $\mathfrak{B} \simeq \mathfrak{A}$, we hope that this will not lead to a confusion.

Obviously, the category $\mathfrak{B}_{0}$ is closed under the tensor product over $\mathbb{C}\left[y, y^{-1}\right]$, and is a Tannakian category over $\mathbb{C}$, with the fiber functor to complex vector spaces given by the fiber at any given non-zero point in the line Spec $\mathbb{C}[y]$. More precisely, we obtain a local system of fiber functors over $\mathbb{C}-\{0\}$. By homotopy invariance, it gives rise to a local system of fiber functors over the circle $S^{1}$ of tangent directions at $\infty \in \mathbb{C P}^{1}$ with coordinate $y$.

Translating everything back by the inverse Fourier transform, we obtain a full-abelian subcategory

$$
\mathfrak{A}_{0} \subset \mathfrak{A}, \quad \operatorname{FT}\left(\mathfrak{A}_{0}\right)=\mathfrak{B}_{0}
$$

equivalent to $\mathfrak{B}_{0}$. This category consists of holonomic $\mathcal{D}$-modules $N$ on $\mathbb{A}_{\mathbb{C}}^{1}$ with regular singularities, such that the operator $d / d x$ is invertible on $N$. In 
other words,

$$
\operatorname{RHom}_{D_{\text {hol }}^{b}\left(\mathcal{D}_{\mathbb{A}_{\mathbb{C}}^{1}}\right)}\left(\mathcal{O}_{\mathbb{A}_{\mathbb{C}}^{1}}, N\right)=H_{D R}^{\bullet}(N)=0 .
$$

The category $\mathfrak{A}_{0}$ is closed under the additive convolution in $D^{b}\left(\mathcal{D}_{\mathbb{A}_{\mathbb{C}}^{1}}-\bmod \right)$ given by

$$
N_{1} *+N_{2}:=\operatorname{sum}_{*}\left(N_{1} \otimes N_{2}\right),
$$

where sum : $\mathbb{C} \times \mathbb{C} \rightarrow \mathbb{C}$ is the addition morphism $\left(x_{1}, x_{2}\right) \mapsto x_{1}+x_{2}$. Moreover, $\mathfrak{A}_{0} \subset \mathfrak{A}$ is the image of an exact idempotent functor $\Pi: \mathfrak{A} \rightarrow \mathfrak{A}$ given by the convolution with $j_{!} \mathcal{O}_{\mathbb{A}_{\mathbb{C}}^{1}-\{0\}}[1]=F T^{-1}\left(\mathbb{C}\left[y, y^{-1}\right]\right)$,

$$
\Pi(N)=N *+j_{!}\left(\mathcal{O}_{\mathbb{A}_{\mathbb{C}}^{1}-\{0\}}[1]\right)
$$

Duality functor on the tensor category $\mathfrak{A}_{0}$ is given by

$$
D_{\mathfrak{A}_{0}} N=\Pi\left(r_{*}\left(D_{\mathcal{D}_{\mathbb{A}_{\mathbb{C}}^{1}}-\bmod } N\right)\right)
$$

where $r: \mathbb{A}_{\mathbb{C}}^{1} \rightarrow \mathbb{A}_{\mathbb{C}}^{1}, r(x)=-x$ is the antipodal map, and $D_{\mathcal{D}_{\mathbb{A}_{\mathbb{C}}^{1}}-\bmod }$ is the standard duality in $D^{b}\left(\mathcal{D}_{\mathbb{A}_{\mathbb{C}}^{1}}-\bmod \right)$.

Tensor category $\mathfrak{A}_{0}$ also admits a local system of fiber functors over the circle $S^{1}$ of tangent directions at the point $\infty$ in the complex line endowed with the coordinate $x$. Namely, any object $N \in \mathfrak{A}_{0}$ gives an analytic $\mathcal{D}$-module $N^{\text {an }}$, which is a complex analytic vector bundle with connection $\nabla$ on a complement to a finite subset of $\mathbb{C}$. The fiber functor associated with an angle $\phi \in \mathbb{R} / 2 \pi \mathbb{Z}$ assigns to $N$ the space of flat sections of $N^{\text {an }}$ on the ray $[R,+\infty) \cdot \exp (\mathrm{i} \phi)$ for sufficiently large $R \gg 0$.

Here we describe a canonical isomorphism between the two local systems of fiber functors: the one described in terms of the category $\mathfrak{A}_{0}$ and another one described in terms of the category $\mathfrak{B}_{0}$. What will follow is basically a reformulation of the classical results of B. Malgrange on the comparison isomorphism between the topological Fourier-Laplace transform and the algebraic Fourier transform for regular holonomic $\mathcal{D}$-modules on $\mathbb{A}^{1}(\mathbb{C})$, see his book [41] devoted to this subject.

For any $N \in \mathfrak{A}$ and a given non-zero point $y_{0} \in \mathbb{C}-\{0\}$ on the dual line, the fiber of $\operatorname{FT}(N)$ at $y_{0}$ is equal to

$$
H_{\mathrm{DR}}^{1}\left(\exp _{y_{0}} \cdot N\right)=\operatorname{Coker}\left(d / d x+y_{0}: N \rightarrow N\right),
$$

where $\exp _{y_{0}}$ is analytic function $x \mapsto \exp \left(x y_{0}\right)$ on $\mathbb{C}$. Suppose that we are given a vector $v$ in this fiber, represented in the de Rham complex by $\exp _{y_{0}} P d x$ for some $P \in N$. Then for any point $x_{t} \in \mathbb{C}$ lying outside of 
singularities of $N$, the same expression $\exp _{y_{0}} P d x$ can be considered as representing a class in

$$
H_{\mathrm{DR}}^{1}\left(\exp _{y_{0}} \cdot N\left[x_{t}\right]\right), \quad N\left[x_{t}\right]:=N \otimes_{\mathbb{C}[x]} \mathbb{C}\left[x, 1 /\left(x-x_{t}\right)\right] .
$$

Passing to analytic functions, we obtain a class in

$$
H_{\mathrm{DR}}^{1}\left(\exp _{y_{0}} \cdot N\left[x_{t}\right]^{\text {an }}\right) \simeq H_{\mathrm{DR}}^{1}\left(N\left[x_{t}\right]^{\text {an }}\right) \simeq H_{\mathrm{DR}}^{1}\left(N\left[x_{t}\right]\right),
$$

where the first isomorphism is given by multiplication by $\exp _{-y_{0}}$, and the second one follows from the assumption that $N$ has regular singularities. Now, if we assume that $N \in \mathfrak{A}_{0}$, i.e., $H_{\mathrm{DR}}^{\bullet}(N)=0$, then $H_{\mathrm{DR}}^{1}\left(N\left[x_{t}\right]\right)$ coincides with the fiber of $N$ at $x_{t}$. Hence, varying $x_{t}$ we obtain a holomorphic section of the $D$-module $N^{\text {an }}$ outside of the set of singularities. This section is not flat, but is exponentially close to a unique flat section along the ray $\mathbb{R}_{\leqslant 0} \cdot y_{0}^{-1}$ at infinity. Moreover, this flat section does not depend on the choice of a representative $P$ of the cohomology class, and gives a vector

$$
v^{\prime} \in \Gamma\left([R,+\infty) \cdot \exp (-\mathrm{i} \phi),\left(N^{\mathrm{an}}\right)^{\nabla}\right)
$$

corresponding to $v$, where $R \gg 0$ and $\phi=\operatorname{Arg}\left(-y_{0}\right)$. The correspondence $v \mapsto v^{\prime}$ gives an identification of two fiber functors evaluated at the object $N$.

An informal meaning of the above construction is that we integrate differential 1-form $\exp _{y_{0}} P d x$ over an appropriate non-compact chain with coefficients in the constructible sheaf associated with the dual object $D_{\mathcal{D}_{A_{\mathrm{C}}^{1}}-\bmod }(N)$ via the Riemann-Hilbert correspondence. The integral is convergent because of the exponential decay of $\exp _{y_{0}}$ along the chain. The role of the point $x_{t}$ in the above construction is to approximate the integral over a non-compact chain by an integral over a compact chain with boundary at $x_{t}$ where the exponential factor $\exp \left(x_{t} y_{0}\right)$ is very small.

Let us apply the Riemann-Hilbert correspondence. The category $\mathfrak{A}$ is equivalent to the abelian category of middle perversity constructible sheaves of $\mathbb{C}$-vector spaces on $\mathbb{A}^{1}(\mathbb{C})$. The subcategory $\mathfrak{A}_{0}$ goes to the full subcategory $\operatorname{Perv}_{0}\left(\mathbb{A}^{1}(\mathbb{C}), \mathbb{C}\right)$ consisting ${ }^{5}$ of objects $E^{\bullet}$ such that $R \Gamma\left(E^{\bullet}\right)=0$. The

\footnotetext{
${ }^{5}$ In $[29]$ the subcategory $\operatorname{Perv}_{0}\left(\mathbb{A}^{1}(\mathbb{C}), \mathbb{C}\right)$ was denoted by $\operatorname{Perv}_{A}(\mathbb{C})$. Moreover, in [30] it was proven that any object of $\mathfrak{A}_{0}$ is in fact a usual constructible sheaf shifted by [1].
} 
additive convolution $*+$

$$
D^{b}\left(\operatorname{Perv}\left(\mathbb{A}^{1}(\mathbb{C}), \mathbb{C}\right)\right) \times D^{b}\left(\operatorname{Perv}\left(\mathbb{A}^{1}(\mathbb{C}), \mathbb{C}\right)\right) \rightarrow D^{b}\left(\operatorname{Perv}\left(\mathbb{A}^{1}(\mathbb{C}), \mathbb{C}\right)\right)
$$

is given by the same formula as for $\mathcal{D}$-modules. Projector $\Pi$ in the category of perverse sheaves of $\mathbb{C}$-vector spaces is given by

$$
\Pi(F)=F *+j !\left(\mathbb{C}_{\mathbb{A}^{1}(\mathbb{C})}[1]\right) .
$$

The conclusion is that the Tannakian category $\mathfrak{B}_{0}$ is equivalent to the abelian Tannakian $\mathbb{C}$-linear category $\operatorname{Perv}_{0}\left(\mathbb{A}^{1}(\mathbb{C}), \mathbb{C}\right)$ ) which is defined purely topologically, endowed with the tensor product given in terms of constructible sheaves. The latter category has a local system of fiber functors over the circle $S^{1}$ about the point $\infty$ given by stalks (shifted by [-1]) at points approaching $\infty$ along straight rays.

It is clear that one can define an exact idempotent functor

$$
\Pi(F)=F *+j !\left(\mathbb{Q}_{\mathbb{A}^{1}(\mathbb{C})}[1]\right)
$$

on the $\mathbb{Q}$-linear category $\operatorname{Perv}_{0}\left(\mathbb{A}^{1}(\mathbb{C}), \mathbb{Q}\right) \subset D^{b}\left(\operatorname{Perv}\left(\mathbb{A}^{1}(\mathbb{C}), \mathbb{Q}\right)\right)$ of perverse sheaves of $\mathbb{Q}$-vector spaces. The convolution on $\operatorname{Perv}_{0}\left(\mathbb{A}^{1}(\mathbb{C}), \mathbb{Q}\right)$ is exact in each argument. Also the category $\operatorname{Perv}_{0}\left(\mathbb{A}^{1}(\mathbb{C}), \mathbb{Q}\right)$ is a Tannakian category, with the local system of fiber functors over $S^{1}$.

Now we can use the theory by M. Saito of mixed Hodge modules (see [49] or [46]). For a smooth complex algebraic variety $X / \mathbb{C}$ we denote by $\mathrm{MHM}_{X}$ the category of mixed Hodge modules on $X$. The category $\mathrm{MHM}_{\mathbb{A}_{\mathbb{C}}^{1}}$ is a $\mathbb{Q}$-linear abelian category endowed with an exact faithful forgetting functor to $\operatorname{Perv}\left(\mathbb{A}^{1}(\mathbb{C}), \mathbb{Q}\right)$ (Betti realization). This category is also endowed with an exact idempotent endofunctor $\Pi$ given by the addtitve convolution with $j_{!}\left(\mathbb{Q}_{\mathbb{A}^{1}(\mathbb{C})}[1]\right)$ considered as an object of $\mathrm{MHM}_{\mathbb{A}_{\mathbb{C}}^{1}}$.

Definition 4.2. The category of exponential mixed Hodge structures EMHS is the full subcategory of the category MHM $_{\mathbb{A}_{\mathbb{C}}^{1}}$ consisting of objects $M$ such that the corresponding perverse sheaf belongs to $\operatorname{Perv}_{0}\left(\mathbb{A}^{1}(\mathbb{C}), \mathbb{Q}\right)$. Equivalently, it is the image of endofunctor $\Pi$. It is a Tannakian category with the tensor product given by the additive convolution.

The tensor category of ordinary mixed Hodge structures $\mathrm{MHM}_{\mathrm{pt}}$ can be identified with a full Serre subcategory of EMHS closed under the tensor product and duality. Namely, with any $M_{0} \in \mathrm{MHM}_{\mathrm{pt}}$ we associate an object $\Pi\left(s_{*} M_{0}\right) \in$ EMHS, where $s:\{0\} \rightarrow \mathbb{A}^{1}(\mathbb{C})$ is the obvious embedding. 


\subsection{Realization functors for exponential mixed Hodge structures}

We have already mentioned that the $\mathbb{C}$-linear tensor category $\mathfrak{A}_{0} \simeq \mathfrak{B}_{0}$ has two canonically equivalent local systems of realization functors, defined in terms of stalks of bundles with connections either for the original holonomic $\mathcal{D}$-module with regular singularity or for its Fourier transform.

Definition 4.3. For an object $N \in$ EMHS and a non-zero complex number $u$ we define de Rham realization of $N$ at the point $u$ to be the fiber of $\mathbb{C}[y]$-module $\mathrm{FT}\left(N_{\mathrm{DR}}^{\mathrm{MHM}}\right)$ at the point $u^{-1} \in \operatorname{Spec} \mathbb{C}[y]$, where $N_{\mathrm{DR}}^{\mathrm{MHM}}$ is the algebraic holonomic $\mathcal{D}$-module underlying $N$, where $N$ is considered as an object of $\mathrm{MHM}_{\mathbb{A}_{\mathbb{C}}^{1}}$. We denote this fiber by $N_{\mathrm{DR}}^{\mathrm{MHM}}$.

It follows directly from the definition that we have a natural isomorphism

$$
\begin{aligned}
& N_{\mathrm{DR}, \mathrm{u}} \simeq H_{\mathrm{DR}}^{1}\left(\mathbb{A}_{\mathbb{C}}^{1}, N_{\mathrm{DR}}^{\mathrm{MHM}} \otimes \exp \left(u^{-1} x\right)\right) \\
& \quad=\operatorname{Coker}\left(d / d x+u^{-1}: N_{\mathrm{DR}}^{\mathrm{MHM}} \rightarrow N_{\mathrm{DR}}^{\mathrm{MHM}}\right) .
\end{aligned}
$$

Varying point $u$ we obtain an algebraic vector bundle of realization functors $\mathrm{DR}_{u}$ with connection over the punctured line of $u \in \mathbb{C}-\{0\}$. The connection has exponential type at $u \rightarrow 0$ and a regular singularity at $u \rightarrow \infty$. It is easy to see that for ordinary mixed Hodge structures (considered as objects of EMHS) the fiber functor $\mathrm{DR}_{u}$ is canonically isomorphic to the usual de Rham realization.

Definition 4.4. For an object $N \in$ EMHS and a non-zero complex number $u$ we define Betti realization of $N$ at point $u$ to be the space of flat sections on the ray $[R,+\infty) \cdot u$ for sufficiently large $R \gg 0$ of the constructible sheaf $N_{\text {Betti }}^{\mathrm{MHM}}[-1]$. Here $N_{\text {Betti }}^{\mathrm{MHM}}$ is the object of $\operatorname{Perv}\left(\mathbb{A}^{1}(\mathbb{C}), \mathbb{Q}\right)$ underlying $N$, where $N$ is considered as an object of $\mathrm{MHM}_{\mathbb{A}_{\mathbb{C}}^{1}}$. We denote this fiber by $N_{\text {Betti,u }}$.

Varying point $u$ we obtain a local system of realization functors Betti $\mathrm{B}_{u}$ in vector spaces over $\mathbb{Q}$. Again, for usual mixed Hodge structures considered as objects of EMHS, realization Betti ${ }_{u}$ has trivial monodromy in parameter $u$ and is canonically isomorphic to the usual Betti realization.

The canonical isomorphism of fiber functors mentioned above gives a comparison isomorphism

$$
N_{\mathrm{DR}, u} \simeq N_{\mathrm{Betti}, u} \otimes_{\mathbb{Q}} \mathbb{C}
$$


extending the standard comparison isomorphism for usual mixed Hodge structures.

Finally, following an idea of M. Saito, one can define an analog of Dolbeault (or Hodge) realization for an object of EMHS. This realization can be considered as a "limit" at $u \rightarrow 0$ of de Rham realizations $\mathrm{DR}_{u}$.

Recall that for any object $M \in \mathrm{MHM}_{X}$ for any smooth algebraic variety $X / \mathbb{C}$ we have a canonical admissible increasing filtration $F_{i} M_{\mathrm{DR}}$ of the underlying algebraic holonomic $\mathcal{D}$-module $M_{\mathrm{DR}}$. In particular, for $X=\mathbb{A}_{\mathbb{C}}^{1}$ the admissibility of filtration means that

$$
(d / d x) F_{i} M_{\mathrm{DR}}=F_{i+1} M_{\mathrm{DR}}
$$

for all $i \gg 0$.

Definition 4.5. For an object $N \in$ EMHS we define complex vector space $N_{\mathrm{DR}, 0}$ to be the quotient

$$
(d / d x)^{-j} F_{j} N_{\mathrm{DR}}^{\mathrm{MHM}} /(d / d x)^{-j} F_{j-1} N_{\mathrm{DR}}^{\mathrm{MHM}}
$$

for sufficiently large $j \gg 0$.

Here we use the fact that the operator $d / d x$ is invertible for $N \in$ EMHS. Equivalently, $N_{\mathrm{DR}, 0}$ is canonically isomorphic (by applying power of $d / d x$ ) to the quotient $F_{j} N_{\mathrm{DR}}^{\mathrm{MHM}} / F_{j-1} N_{\mathrm{DR}}^{\mathrm{MHM}}$ for large $j \gg 0$.

For the usual mixed Hodge structures the realization $\mathrm{DR}_{0}$ is canonically isomorphic to the usual Dolbeault/Hogde realization, i.e., to the functor

$$
M \in \mathrm{MHM}_{\mathrm{pt}} \mapsto \oplus_{i} \operatorname{gr}_{\mathcal{F}}^{i} M_{\mathrm{DR}}
$$

where $\mathcal{F}$ is the usual (decreasing) Hodge filtration.

The following result is not difficult to prove. Since we will not use it, the proof is omitted.

Theorem 4.1. Functor $D R_{0}$ from EMHS to the category of $\mathbb{C}$-vector spaces is exact, faithful and commutes with the tensor product.

One can see easily that functors $\mathrm{DR}_{u}$ for $u \neq 0$ and $\mathrm{DR}_{0}$ glue together to an algebraic vector bundle of fiber functors on EMHS over the affine line Spec $\mathbb{C}[u]$, with flat connection having pole of order 2 at $u=0$. Hence, we obtain a so-called nc Hodge structure in the sense of [30], i.e., a germ of a holomorphic vector bundle near $0 \in \mathbb{C}$ together with a meromorphic 
connection with second-order pole at 0 , and a rational structure on the corresponding local system on the circle $S^{1}$ of directions about the point 0 . Moreover, all terms of the Stokes filtration are rational vector subspaces with respect to the $\mathbb{Q}$-structure.

We should warn the reader that there are natural examples of nc Hodge structures appearing in mirror symmetry and in singularity theory which $d o$ not come from EMHS. In particular, for a general (not quasi-homogeneous) isolated singularity of a holomorphic function the corresponding nc Hodge structure does not come from an exponential one. For example, it is easy to construct a non-trivial family of isolated singularities with constant Milnor number over $\mathbb{A}_{\mathbb{C}}^{1}$, giving a non-trivial variation of non-commutative Hodge structures $^{6}$. On other side, any variation of EMHS over $\mathbb{C}^{1}$ (with tame singularity at infinity) is trivial. Similarly, for the nc Hodge structure associated with a non-Kähler compact symplectic manifold with symplectic form rescaled by a sufficiently large factor, there is no reasons to expect that there exists an underlying object of EMHS.

\subsection{Weight filtration in the exponential case}

Let us denote by $i$ the natural inclusion functor EMHS $\hookrightarrow M M_{\mathbb{A}_{c}^{1}}$ and by $p: \operatorname{MHM}_{\mathbb{A}_{\mathrm{C}}^{1}} \rightarrow$ EMHS its left adjoint. Both functors are exact, and

$$
p \circ i=\operatorname{id}_{\mathrm{EMHS}}, \quad i \circ p=\Pi,
$$

where we use the same notation $\Pi$ (as for categories $\mathfrak{A} \simeq \operatorname{Perv}\left(\mathbb{A}_{\mathbb{C}}^{1}\right)$ ) for an exact idempotent functor of $\mathrm{MHM}_{\mathbb{A}_{\mathbb{C}}^{1}}$ given by

$$
\Pi: M \mapsto M *_{+} j_{!}\left(\mathbb{Q}(0)_{\mathbb{A}_{\mathbb{C}}^{1}}[1]\right) .
$$

Definition 4.6. The weight filtration of an exponential mixed Hodge structure is defined by the formula

$$
W_{\leqslant n}^{\mathrm{EMHS}} M=W_{n}^{\mathrm{EMHS}} M:=p\left(W_{n}(i(M))\right),
$$

where in the RHS we consider the usual weight filtration for mixed Hodge modules.

\footnotetext{
${ }^{6}$ See e.g. Section 3.3 in the third part of [36], on the period map for $E_{12}$ singularities. In this book the nc Hodge structure for an isolated singularity is encoded by the equivalent notion of a Brieskorn lattice.
} 
Proposition 4.1. The endofunctor $\mathrm{gr}^{W, E M H S}$ of EMHS obtained by taking the associated graded object with respect to the filtration $W_{\bullet}^{E M H S}$ is exact and faithful.

Proof. The exactness follows from exactness of functors $i, p$, and of the endofunctor $\mathrm{gr}_{\bullet}^{W}$ of $\mathrm{MHM}_{\mathbb{A}_{\mathbb{C}}^{1}}$. To prove the faithfulness we use the existence of the fiber functor on the tensor category EMHS. Namely, any object $M \in$ EMHS has $\operatorname{rk}(M) \geqslant 0$, and $M=0$ if and only if $\operatorname{rk}(M)=0$. Endofunctor $\operatorname{gr}_{\bullet}^{W, \text { EMHS }}$ preserves the rank because

$$
W_{n}^{\mathrm{EMHS}} M=0 \text {, for } n \ll 0, \quad W_{n}^{\mathrm{EMHS}} M=M \text {, for } n \gg 0 \text {. }
$$

Let $\alpha: M \rightarrow N$ be a morphism such that $\operatorname{gr}_{\bullet}^{W \text {,EMHS }}(\alpha)=0$. The exactness of $\mathrm{gr}_{\bullet}^{W, \text { EMHS }}$ implies that

$$
\operatorname{gr}_{\bullet}^{W, \operatorname{EMHS}}(\operatorname{Ker} \alpha)=\operatorname{Ker}\left(\operatorname{gr}_{\bullet}^{W, \text { EMHS }} \alpha\right)=\operatorname{gr}_{\bullet}^{W, \operatorname{EMHS}}(M) .
$$

Hence the rank of $\operatorname{Ker} \alpha$ is equal to the rank of $M$, and we conclude that $\operatorname{rk}(M / \operatorname{Ker} \alpha)=0$. Therefore $M=\operatorname{Ker} \alpha$ and $\alpha=0$.

Definition 4.7. An object $M \in$ EMHS is called pure of weight $n \in \mathbb{Z}$ if $W_{n}^{\text {EMHS }} M=M$ and $W_{n-1}^{\text {EMHS }} M=0$.

Graded factors of the weight filtration of an object in EMHS are pure. The next proposition describes the structure of pure objects in EMHS.

Proposition 4.2. The full subcategory of direct sums of pure objects in EMHS is a semisimple abelian category. Moreover, it is equivalent to a direct summand of the semisimple category of pure objects in $M H M_{\mathbb{A}_{\mathbb{C}}^{1}}$, with the complement consisting of constant variations of pure Hodge structures on $\mathbb{A}^{1}(\mathbb{C})$.

Proof. Obviously, the image of any pure $F \in \mathrm{MHM}_{\mathbb{A}_{\mathbb{C}}^{1}}$ of weight $n \in \mathbb{Z}$ by projection $p$ is a pure object of EMHS of the same weight. Conversely, any pure object $E \in$ EMHS of weight $n$ is the image of a pure object of weight $n$ in $\mathrm{MHM}_{\mathbb{A}_{\mathbb{C}}^{1}}$. Indeed, we have

$$
p\left(W_{n}(i(E))\right)=E, \quad p\left(W_{n-1}(i(E))\right)=0
$$

and by exactness of $p$ we conclude that

$$
E=p\left(W_{n}(i(E)) / W_{n-1}(i(E))\right)=p\left(\operatorname{gr}_{n}^{W}(i(E))\right)
$$


Also, it is easy to see that the kernel of the projector $\Pi$ on $\operatorname{Perv}\left(\mathbb{A}^{1}(\mathbb{C}), \mathbb{Q}\right)$ consists of constant sheaves. Any pure object of $\mathrm{MHM}_{\mathbb{A}_{\mathbb{C}}^{1}}$ whose underlying perverse sheaf is constant is a constant variation of pure Hodge structure. Hence, we conclude that the set of isomorphism classes of pure objects of weight $n$ in EMHS is in one-to-one correspondence with pure objects of weight $n$ in $\mathrm{MHM}_{\mathbb{A}_{\mathbb{C}}^{1}}$, which have no direct summands which are constant variations.

For any pure object $F$ of weight $n$ in EMHS without constant direct summand the corresponding pure object $E=p(F)$ has the following structure. The corresponding object $i(E)=\Pi(F)$ has two-step weight filtration:

$$
\Pi(F)=W_{n} \Pi(F) \supset W_{n-1} \Pi(F) \supset W_{n-2} \Pi(F)=0
$$

with $\operatorname{gr}_{n}^{W} \Pi(F)=F$ and $\operatorname{gr}_{n-1}^{W} \Pi(F)$ being a constant variation of Hodge structures of weight $n-1$.

We have to check for any two pure objects $E_{1}, E_{2} \in$ EMHS of weights $n_{1}, n_{2} \in \mathbb{Z}$ the following isomorphism holds:

$$
\operatorname{Hom}\left(E_{1}, E_{2}\right) \simeq \operatorname{Hom}\left(\operatorname{gr}_{n_{1}}^{W}\left(i\left(E_{1}\right)\right), \operatorname{gr}_{n_{2}}^{W}\left(i\left(E_{2}\right)\right)\right)
$$

First, in the case $n_{1} \neq n_{2}$ the LHS vanishes by the faithfulness of the functor $\mathrm{gr}_{\bullet}^{W, \text { EMHS }}$, and the RHS vanishes by the faithfulness of $\mathrm{gr}_{\bullet}^{W}$.

Consider now the case of equal weights $n_{1}, n_{2}$ and denote $F_{j}:=$ $\operatorname{gr}_{n}^{W}\left(i\left(E_{j}\right)\right), j=1,2$ the corresponding pure objects of $\mathrm{MHM}_{\mathbb{A}_{\mathbb{C}}^{1}}$. Adjunction morphisms

$$
F_{i} \rightarrow \Pi\left(F_{i}\right), \quad \Pi=i \circ p
$$

induce (after composition with $\operatorname{gr}_{\bullet}^{W}$ ) isomorphisms $F_{j} \simeq \operatorname{gr}_{n}^{W}\left(\Pi\left(F_{j}\right)\right.$ ). We have

$$
\begin{aligned}
& \operatorname{Hom}\left(E_{1}, E_{2}\right) \simeq \operatorname{Hom}\left(p\left(F_{1}\right), p\left(F_{2}\right)\right) \simeq \operatorname{Hom}\left(F_{1}, \Pi\left(F_{2}\right)\right) \\
& \quad \hookrightarrow \operatorname{Hom}\left(\operatorname{gr}_{\bullet}^{W}\left(F_{1}\right), \operatorname{gr}_{\bullet}^{W}\left(\Pi F_{2}\right)\right) \simeq \operatorname{Hom}\left(F_{1}, \operatorname{gr}_{n}^{W} i\left(E_{2}\right)\right) \simeq \operatorname{Hom}\left(F_{1}, F_{2}\right)
\end{aligned}
$$

by faithfulness of the functor $\operatorname{gr}_{\bullet}^{W}$. Hence $\operatorname{rk} \operatorname{Hom}\left(E_{1}, E_{2}\right) \leqslant \operatorname{rk} \operatorname{Hom}\left(F_{1}, F_{2}\right)$. On the other hand, we have natural transformations of functors

$$
\operatorname{id}_{\mathrm{MHM}_{\mathbb{A}_{\mathbb{C}}^{1}}} \rightarrow \Pi \rightarrow \Pi /\left(W_{n-1} \circ \Pi\right)
$$

which induce isomorphisms

$$
F_{j} \rightarrow \Pi\left(F_{j}\right) \rightarrow \Pi\left(F_{j}\right) / W_{n-1} \Pi\left(F_{j}\right) \simeq \operatorname{gr}_{n}^{W}\left(\Pi\left(F_{j}\right)\right) \simeq F_{j}
$$


Hence $\operatorname{Hom}\left(F_{1}, F_{2}\right)$ is a retract of $\operatorname{Hom}\left(\Pi\left(F_{1}\right), \Pi\left(F_{2}\right)\right) \simeq \operatorname{Hom}\left(E_{1}, E_{2}\right)$. We conclude that $\operatorname{Hom}\left(F_{1}, F_{2}\right) \simeq \operatorname{Hom}\left(E_{1}, E_{2}\right)$.

Proposition 4.3. The weight filtration on EMHS is strictly compatible with the tensor product.

Proof. We start with the following lemma.

Lemma 4.1. Let $E, F \in D^{b}\left(M H M_{\mathbb{A}_{\mathbb{C}}^{1}}\right)$ and

$$
G=\operatorname{Cone}\left(\operatorname{sum}_{!}(E \otimes F) \rightarrow \operatorname{sum}_{*}(E \otimes F)\right)
$$

Then $\Pi(G)=0$.

Proof of the Lemma. We first observe that we can assume that $E$ and $F$ are just constructible sheaves (since the forgetful functor to constructible sheaves is faithful). We will show that $G$ is a constant sheaf. The fiber of $G$ at $x \in \mathbb{A}^{1}(\mathbb{C})$ is canonically isomorphic to $H^{\bullet}\left(S_{x, R}^{1}, E \otimes F\right)$, where $S_{x, R}^{1} \subset$ $\operatorname{sum}^{-1}(x)$ is a circle of a sufficiently large radius $R$ with any given center. Obviously the cohomology groups do not depend on $x$. Hence, they form a constant sheaf on $\mathbb{A}^{1}(\mathbb{C})$. In order to finish the proof of the lemma we observe that the functor $\Pi$ kills all constant sheaves on $\mathbb{A}^{1}(\mathbb{C})$.

Let us return to the proof of the proposition. It is enough to prove that pure objects in EMHS are closed under the tensor product, and weights behave additively.

Assume that $E_{j} \in$ EMHS, $j=1,2$ are objects of pure weights $n_{k}, k=$ 1,2 . Then $E_{j}=p\left(F_{j}\right)$ for some pure objects $F_{j}$ in $\mathrm{MHM}_{\mathbb{A}_{\mathbb{C}}^{1}}, j=1,2$. In the category EMHS we have:

$$
E_{1} \otimes_{\mathrm{EMHS}} E_{2}=p\left(\operatorname{sum}_{*}\left(F_{1} \otimes F_{2}\right)\right)=p\left(\operatorname{sum}_{!}\left(F_{1} \otimes F_{2}\right)\right)
$$

where the second equality follows from the lemma. Since both objects $F_{1}$ and $F_{2}$ are pure, we see that $F_{1} \otimes F_{2}$ is pure. It is well known (see e.g. [46]) that $\operatorname{sum}_{*}\left(F_{1} \otimes F_{2}\right)$ has weights (in the derived sense) greater or equal than $n_{1}+n_{2}$ while $\operatorname{sum}_{!}\left(F_{1} \otimes F_{2}\right)$ has weights less or equal than $n_{1}+n_{2}$.

Let us denote by $H_{\text {perv }}^{0}(F)$ the zeroth middle perverse cohomology of an object $F \in D^{b}\left(\mathrm{MHM}_{\mathbb{A}_{\mathbb{C}}^{1}}\right)$. Then

$$
p \circ \operatorname{sum}_{*}\left(F_{1} \otimes F_{2}\right)=p \circ\left(H_{\text {perv }}^{0}\left(\operatorname{sum}_{*}\left(F_{1} \otimes F_{2}\right)\right),\right.
$$


and similarly for $\operatorname{sum}_{!}\left(F_{1} \otimes F_{2}\right)$ (recall that the functor $p$ is exact). Therefore,

$$
E_{1} \otimes_{\mathrm{EMHS}} E_{2}=p\left(H_{\text {perv }}^{0}\left(\operatorname{sum}_{*}\left(F_{1} \otimes F_{2}\right)\right)\right)=p\left(H_{\text {perv }}^{0}\left(\operatorname{sum}_{!}\left(F_{1} \otimes F_{2}\right)\right)\right) .
$$

We see that $E_{1} \otimes_{\mathrm{EMHS}} E_{2}$ is the image by $p$ of an object of $\mathrm{MHM}_{\mathbb{A}_{\mathbb{C}}^{1}}$ of the weight $\geqslant\left(n_{1}+n_{2}\right)$. But it is also the image by $p$ of an object of the weight $\leqslant\left(n_{1}+n_{2}\right)$. Notice that the exact functor $p$ maps weight filtration in $\mathrm{MHM}_{\mathbb{A}_{\mathbb{C}}^{1}}$ to the weight filtration in EMHS, as follows from our description of pure objects in EMHS. Therefore $E_{1} \otimes_{\text {EMHS }} E_{2}$ is pure of weight $n_{1}+n_{2}$. This concludes the proof of the proposition.

Let us define 'Serre polynomial' of an exponential mixed Hodge structure $E$ as

$$
S(E)=\sum_{i} r k \operatorname{gr}_{i}^{\mathrm{W}, \mathrm{EMHS}}(E) q^{i / 2} \in \mathbb{Z}\left[q^{ \pm 1 / 2}\right]
$$

By additivity we extend $S$ to a functional on the $K_{0}$-group of the bounded derived category of EMHS. By Proposition 4.3 the map $S$ is a ring homomorphism.

\subsection{Cohomology of a variety with function}

For any separated scheme of finite type $X / \mathbb{C}$ and a function $f \in \mathcal{O}(X)$ (sometimes called potential) we define exponential cohomology (which will be an exponential mixed Hodge structure) $H_{\mathrm{EMHS}}^{i}(X, f)$ for integers $i \geqslant 0$ in the following way:

$$
H_{\mathrm{EMHS}}^{i}(X, f):=\Pi\left(H_{\mathrm{MHM}_{\mathbb{A}_{\mathbb{C}}^{1}}}^{i} f_{*} \mathbb{Q}_{X}\right)=H_{\mathrm{MHM}_{\mathbb{A}_{\mathbb{C}}^{1}}}^{i}\left(\Pi f_{*} \mathbb{Q}_{X}\right) \in \mathrm{EMHS},
$$

where we interchange $\Pi$ and cohomology in $D^{b}\left(\mathrm{MHM}_{\mathbb{A}_{\mathbb{C}}^{1}}\right)$ using the exactness of the functor $\Pi$.

If $f$ is the restriction of a function $f^{\prime}$ defined on a bigger variety $X^{\prime} \supset X$ then we will abuse the notation denoting both functions by the same symbol. The rapid decay cohomology of the pair $(X, f)$ does not depend on such an extension. We will see in Section 7 that for the so-called critical cohomology (defined in terms of the vanishing cycles functor) the situation is different, and the result depends on the extension of the function. 
As follows directly from definitions, the Betti realization of $H_{\mathrm{EMHS}}^{i}(X, f)$ is given by the cohomology of pair

$$
\begin{aligned}
& H_{\mathrm{Betti}, u}^{i}(X, f):=H_{\mathrm{Betti}}^{i}\left(X(\mathbb{C}), f^{-1}(-c \cdot u) ; \mathbb{Q}\right), \\
& H_{\text {Betti }}^{i}(X, f):=H_{\text {Betti }, 1}^{i}(X, f),
\end{aligned}
$$

where $c \gg 0$ is a sufficiently large positive real number. Cohomology groups are identified for different large values of $c$ via the holonomy along the ray $\mathbb{R}_{<0} \cdot u$.

We define Betti cohomology with integer coefficients as

$$
H_{u}^{i}(X, f):=H^{i}\left(X(\mathbb{C}), f^{-1}(-c \cdot u) ; \mathbb{Z}\right), H_{\operatorname{Betti}, u}^{i}(X, f) \simeq H_{u}^{i}(X, f) \otimes_{\mathbb{Z}} \mathbb{Q}
$$

The cohomology $H^{\bullet}(X, f):=H_{1}^{\bullet}(X, f)$ behaves similarly to the usual cohomology. In particular, for a map $\pi: Y \rightarrow X$ compatible with functions $f_{Y} \in$ $\mathcal{O}(Y), f_{X} \in \mathcal{O}(X)$ in the sense that $f_{Y}=\pi^{*} f_{X}$, we have the pullback $\pi^{*}$ : $H^{\bullet}\left(X, f_{X}\right) \rightarrow H^{\bullet}\left(Y, f_{Y}\right)$. When $\pi$ is proper and both $X$ and $Y$ are smooth we have the pushforward morphism $\pi_{*}: H^{\bullet}\left(Y, f_{Y}\right) \rightarrow H^{\bullet+2}\left(\operatorname{dim}_{\mathbb{C}}(X)-\operatorname{dim}_{\mathbb{C}}(Y)\right)$ $\left(X, f_{X}\right)$. All these morphisms induce morphisms of exponential mixed Hodge structures (for the pushforward we should twist by a power of the Tate module $\mathbb{T}$ ). The proof is a straightforward application of the standard formalism of six functors.

As for usual cohomology, there is a multiplication morphism

$$
\otimes: H^{\bullet}\left(X, f_{X}\right) \otimes H^{\bullet}\left(Y, f_{Y}\right) \rightarrow H^{\bullet}\left(X \times Y, f_{X} \boxplus f_{Y}\right)
$$

where the Thom-Sebastiani sum $\boxplus$ is given by

$$
f_{X} \boxplus f_{Y}:=\operatorname{pr}_{X \times Y \rightarrow X}^{*} f_{X}+\operatorname{pr}_{X \times Y \rightarrow Y}^{*} f_{Y}
$$

This morphism is well-defined because when the real part of the sum of two functions tends to $-\infty$, then the real part of at least one of the functions also tends to $-\infty$. The multiplication morphism becomes an isomorphism after the extension of coefficients for cohomology from $\mathbb{Z}$ to $\mathbb{Q}$. The multiplication morphism gives an isomorphism of exponential mixed Hodge structures

$$
H_{\mathrm{EMHS}}^{\bullet}\left(X, f_{X}\right) \otimes H_{\mathrm{EMHS}}^{\bullet}\left(Y, f_{Y}\right) \stackrel{\sim}{\rightarrow} H_{\mathrm{EMHS}}^{\bullet}\left(X \times Y, f_{X} \boxplus f_{Y}\right)
$$

For smooth $X$ the de Rham realization of $H_{\text {EMHS }}^{i}(X, f)$ (at a point $\left.u \in \mathbb{C}^{*}\right)$ is given by the de Rham cohomology of $X$ with coefficients in 
the holonomic $\mathcal{D}_{X}$-module $\exp \left(u^{-1} \cdot f\right) \cdot \mathcal{O}_{X}$. In other words, it is a finitedimensional $\mathbb{Z}$-graded vector space over $\mathbb{C}$, which is the hypercohomology in Zariski topology

$$
H_{\mathrm{DR}, u}^{\bullet}(X, f):=\mathbb{H}^{\bullet}\left(X_{\mathrm{Zar}},\left(\Omega_{X}^{\bullet}, d+u^{-1} d f \wedge \cdot\right)\right), H_{\mathrm{DR}}^{\bullet}(X, f):=H_{\mathrm{DR}, 1}^{\bullet}(X, f) .
$$

The abstract comparison isomorphism between complexified Betti and de Rham realization (see Section 4.3, and also Theorem 1.1 in [48] and references therein) is

$$
H_{\mathrm{DR}}^{\bullet}(X, f) \simeq \mathbb{C} \otimes H^{\bullet}(X, f)
$$

In the affine case it is given by the integration of complex-analytic closed forms on $X(\mathbb{C})$ of the type $\exp (f) \alpha$, where $\alpha$ is an algebraic form on $X$ such that

$$
d \alpha+d f \wedge \alpha=0 \Longleftrightarrow d(\exp (f) \alpha)=0,
$$

over closed real semi-algebraic chains with the "boundary at infinity" in the direction $\operatorname{Re}(f) \rightarrow-\infty$. The integral is absolutely convergent because the form $\exp (f) \alpha$ decays rapidly at infinity. This explains the term "rapid decay cohomology".

An important example of a (graded) EMHS is

$$
\mathbb{T}^{\otimes 1 / 2}:=H^{\bullet}\left(\mathbb{A}_{\mathbb{C}}^{1},-z^{2}\right)
$$

which is an odd one-dimensional space in degree +1 corresponding to the period integral $\int_{-\infty}^{+\infty} \exp \left(-z^{2}\right) d z=\sqrt{\pi}$. This structure is a "square root" of the shifted Tate structure $\mathbb{T}=\mathbb{Q}(-1)[-2]$, and is a pure EMHS of weight +1 and degree +1 .

Notice that de Rham cohomology can be defined for a pair $(X, f)$ where $X$ is a smooth scheme over arbitrary field of characteristic zero, and $f \in$ $\mathcal{O}(X)$. If $\left(X_{0}, f_{0}\right)$ is defined over $\mathbb{Q}$ then the comparison isomorphism (say, at the point $u=1$ ) gives two rational structures on the same complex vector space. These two structures are related by a period matrix whose coefficients are exponential periods in the sense of Section 4.3 in [35].

One can also define the exponential cohomology with compact support by

$$
H_{c, \text { EMHS }}^{\bullet}(X, f):=\Pi\left(H_{\mathrm{MHMA}_{\mathbb{C}}^{1}}^{\bullet} f_{!} \mathbb{Q}_{X}\right) .
$$

They satisfy the following Poincaré duality for smooth $X$ of dimension $d$ :

$$
H_{c, \mathrm{EMHS}}^{\bullet}(X, f)^{\vee} \simeq H_{\mathrm{EMHS}}^{\bullet}(X,-f) \otimes \mathbb{Q}(d)[2 d]
$$


Also, one can define exponential cohomology of pairs by

$$
H_{\mathrm{EMHS}}^{\bullet}(X, Y, f):=\Pi\left(H_{\mathrm{MHM}_{\mathbb{A}_{\mathbb{C}}^{1}}} f_{*} \operatorname{Cone}\left(\mathbb{Q}_{X} \rightarrow(Y \rightarrow X)_{*} \mathbb{Q}_{Y}\right)\right)[-1] .
$$

For a smooth closed $Z \subset X$ of codimension $d^{\prime}$ in smooth $X$ we have the Thom isomorphism

$$
H_{\mathrm{EMHS}}^{\bullet}(X, X-Z, f) \simeq H_{\mathrm{EMHS}}^{\bullet}(Z, f) \otimes \mathbb{Q}\left(d^{\prime}\right)\left[2 d^{\prime}\right] .
$$

If affine algebraic group $G$ acts on a scheme of finite type $X$ preserving $f \in \mathcal{O}(X)$, we define equivariant exponential cohomology as

$$
H_{G, \mathrm{EMHS}}^{\bullet}(X, f):=H_{\mathrm{EMHS}}^{\bullet}\left(X^{\text {univ }}, f^{\text {univ }}\right) \text {, }
$$

where $X^{\text {univ }}$ is the universal bundle over B $G$ endowed with the induced function $f^{\text {univ }}$. This cohomology depends only on the quotient stack $X / G$ and on $f \in \mathcal{O}(X / G)$, so we will sometimes denote it by $H_{\mathrm{EMHS}}^{\bullet}(X / G, f)$.

We will also need exponential cohomology with compact support, which can be defined for a quotient Artin stack endowed with a function in the following way. We can always assume that our stack is represented as $X / G$ where $G=\operatorname{GL}(n, \mathbb{C})$ for some $n$. The standard classifying space $\mathrm{B} G=$ $\underset{\lim }{\longrightarrow}(\mathrm{B} G)_{N}$ (where $N \rightarrow \infty$ ) is the union of finite-dimensional Grassman$\overrightarrow{\text { nians }}(\mathrm{B} G)_{N}:=\operatorname{Gr}\left(n, \mathbb{C}^{N}\right)$ which are compact smooth varieties. Then we have an inductive system $X_{N} \rightarrow(\mathrm{B} G)_{N}$ of locally trivial bundles endowed with functions $f_{N}$. Then we define the exponential cohomology with compact support of the stack $X / G$ with function $f$ as

$$
\left.H_{\mathrm{c}, \mathrm{EMHS}}^{\bullet}(X / G, f):=\underset{N}{\lim _{\mathrm{C}, \mathrm{EMHS}}} H_{N}^{\bullet}, f_{N}\right) \otimes \mathbb{T}^{\otimes-\operatorname{dim}\left((\mathrm{B} G)_{N} \times G\right)},
$$

where connecting maps are Gysin morphisms. Cohomology groups $H_{c \text {,EMHS }}^{\bullet}(X / G, f)$ stabilize in each degree and are concentrated in cohomological degrees bounded from above. For smooth equidimensional $X$ cohomology groups $H_{\mathrm{EMHS}}^{\bullet}(X / G, f)$ and $H_{c, \mathrm{EMHS}}^{\bullet}(X / G,-f)$ satisfy Poincaré duality in dimension $\operatorname{dim}(X / G):=\operatorname{dim}(X)-\operatorname{dim}(G)^{7}$. In what follows, we will often omit the word "exponential" if it is clear that we are talking about exponential cohomology.

Definition 4.8. A $\mathbb{Z}$-graded object $E^{\bullet}=\oplus_{n \in \mathbb{Z}} E^{n}$ of EMHS is called pure if for any $n \in \mathbb{Z}$ component $E^{n}$ is pure of weight $n$.

\footnotetext{
${ }^{7}$ Cohomology of Artin stacks are discussed in different settings and approaches in e.g. $[2,8,38]$. We use here a more direct approach that is sufficient for our purposes.
} 
Similarly to the classical situation without potential we have

Proposition 4.4. For a pair $(X, f)$ over $\mathbb{C}$ such that $X$ is smooth and the natural morphism $H_{c, E M H S}^{\bullet}(X, f) \rightarrow H_{E M H S}^{\bullet}(X, f)$ is an isomorphism, the cohomology $H_{c, E M H S}^{\bullet}(X, f)$ is pure.

Proof. This follows from the fact that $f_{*} \mathbb{Q}_{X} \in D^{b}\left(M H M\left(\mathbb{A}_{\mathbb{C}}^{1}\right)\right)$ has weights $\geqslant 0$ (in the derived sense), while $f_{!} \mathbb{Q}_{X}$ has weights $\leqslant 0$.

In particular, the conditions of the Proposition hold for a pair $(X, f)$ such that $X$ is smooth and $f: X \rightarrow \mathbb{A}_{\mathbb{C}}^{1}$ is proper. More generally, $f$ could be cohomologically tame (see e.g. $[12,44]$ ), e.g. when $X$ is an affine space and $f$ is a quasi-homogeneous polynomial with isolated singularity. For example, the graded EMHS given by $\mathbb{T}^{\otimes 1 / 2}$ is pure.

If $H_{\mathrm{EMHS}}^{\bullet}(X, f)$ is pure then the Serre polynomial

$$
S\left(H_{\mathrm{EMHS}}^{\bullet}(X, f)\right)=\sum_{n \in \mathbb{Z}}(-1)^{n} S\left(H_{\mathrm{EMHS}}^{n}(X, f)\right) \in \mathbb{Z}\left[q^{1 / 2}\right]
$$

coincides with the Poincaré polynomial of $H_{\mathrm{Betti}}^{i}\left(X(\mathbb{C}), f^{-1}(c \cdot u) ; \mathbb{Q}\right)$ in variable $\left(-q^{1 / 2}\right)$, and the evaluation at $q^{1 / 2}=1$ coincides with the Euler characteristic

$$
\chi(X, f):=\chi\left(H_{\mathrm{EMHS}}^{\bullet}(X, f)\right) .
$$

Using the purity of $H_{\mathrm{EMHS}}^{\bullet}(X, f)$ for smooth $X$ and proper $f$, we can obtain an elementary topological description of the weight filtration in the more general case when $X$ is smooth but $f$ is not necessarily proper. Namely, for any smooth $X / \mathbb{C}$ and $f \in \mathcal{O}(X)$ there exist smooth $\bar{X} \supset X$ and $f^{\prime} \in$ $\mathcal{O}(\bar{X})$ such that $f_{\mid X}^{\prime}=f$, the map $f^{\prime}: \bar{X} \rightarrow \mathbb{A}_{\mathbb{C}}^{1}$ is proper, and the complement $D=\bar{X}-X$ is a divisor with normal crossings. Then the term $W_{k}$ of the weight filtration on $H_{\mathrm{EMHS}}^{i}(X, f)$ is given by the image of $H_{\mathrm{EMHS}}^{i}\left(X^{(j)}\right.$, $\left.f_{\mid X^{(j)}}^{\prime}\right)$ where $X^{(j)}$ is the complement in $\bar{X}$ to the union of $j$-dimensional strata in $D$, for $j=k-i-1$. The same is true for $H_{\mathrm{DR}}^{\bullet}$ and $H_{\mathrm{Betti}}^{\bullet}$.

Also, similarly to the case of usual mixed Hodge structures, one can describe the associated graded space with respect to the weight filtration entirely in terms of pure structures. For that purpose, it is more convenient to use cohomology with compact support. Let us make a simplifying assumption that $D=\bar{X}-X$ is the union of smooth divisors $D_{1}, \ldots, D_{N}$ intersecting transversally. Then $\operatorname{gr}_{k}^{W} H_{c, \text { Betti }}^{l}(X, f)$ is isomorphic to the cohomology 
group in degree $n=l-k$ of the complex

$$
\bigoplus_{0 \leqslant n \leqslant N} \bigoplus_{1 \leq i_{1}<\cdots<i_{n} \leqslant N} H_{\mathrm{Betti}}^{k}\left(D_{i_{1}} \cap \cdots \cap D_{i_{n}}, f\right)
$$

with the differential given by the restriction morphisms.

\section{6. Étale case}

Let $\mathbf{k}$ be any field and $l$ be a prime, $l \neq \operatorname{char}(\mathbf{k})$. With any scheme of finite type $X / \mathbf{k}$ and $f \in \mathcal{O}(X)$ we associate perverse $l$-adic sheaves on $\mathbb{A}_{\mathbf{k}}^{1}$ by the formula

$$
H_{l}^{i}(X, f):=\Pi\left(H_{\operatorname{Perv}_{l} \mathbb{A}_{\mathbf{k}}^{1}}^{i} f_{*} \mathbb{Q}_{l, X}\right),
$$

where the functor $\Pi$ on the category $\operatorname{Perv}_{l} \mathbb{A}_{\mathbf{k}}^{1}$ of $l$-adic perverse sheaves on $\mathbb{A}_{\mathbf{k}}^{1}$ is defined similarly to the constructible case and $\mathbf{k}=\mathbb{C}$. These sheaves are killed by the functor $\mathrm{R} \Gamma$.

If char $\mathbf{k}=0$ then by the comparison isomorphism we obtain just another realization of the "exponential motive" associated with $(X, f)$. All previous considerations concerning the weight filtration hold in $l$-adic case as well. In the case $\mathbf{k}=\mathbb{Q}$ one can define an exponential motivic Galois group (see Chapter 4 and in particular Section 4.3 in [35]) whose representations have both $l$-adic and EMHS realizations.

The situation is more complicated if $p:=$ char $\mathbf{k}$ is non-zero. In this case one can apply Fourier transform using the standard exponential sheaf depending on the choice of a primitive $p$ th root of unity in $\overline{\mathbb{Q}}_{l}$.

The restriction of $\mathrm{FT}\left(f_{*} \mathbb{Q}_{l, X}\right)$ to $\mathbb{A}_{\mathbf{k}}^{1}-\{0\}$ coincides with $\mathrm{FT}\left(\Pi\left(f_{*} \mathbb{Q}_{l, X}\right)\right)$. Perverse sheaves $\mathrm{FT}\left(H_{l}^{i}(X, f)\right)$ are in general not lisse.

Picking any non-zero point $u$ in $\mathbb{A}^{1}(\mathbf{k})$ (e.g. point $u=1$ ) one obtains a cohomology theory for pairs $(X, f)$ with values in $\overline{\mathbb{Q}}_{l}$-linear representations of $\mathrm{Gal}\left(\mathbf{k}^{\mathrm{sep}} / \mathbf{k}\right)$, by taking $H^{i}$ of the fiber of $\mathrm{FT}\left(f_{*} \mathbb{Q}_{l, X}\right)$ at $u$. For this cohomology theory (which one can denote by $H_{l, u}^{\bullet}(X, f)$ ) one can define the weight filtration, Serre polynomial, and also a multiplicative $\overline{\mathbb{Q}}_{l}$-valued numerical invariant (in fact it is a $\mathbb{Q}(\exp (2 \pi \mathrm{i} / p)$-valued invariant) obtained by taking the supertrace of Frobenius.

For $X=\mathbb{A}_{\mathbf{k}}^{1}$ and any $\lambda \in \mathbf{k}^{*}$ the exponential l-adic structure $\otimes \mathbb{T}_{\lambda, l}^{1 / 2}$ corresponding to $\left(\mathbb{A}_{\mathbf{k}}^{1}, \lambda z^{2}\right)$, where $z$ is a coordinate on $\mathbb{A}_{\mathbf{k}}^{1}$, is a tensor square root of $\mathbb{T}=\mathbb{T}_{l}=\mathbb{Q}_{l}(-1)[-2]$, if and only if $\mathbf{k}$ contains $\sqrt{-1}$. Different choices of $\lambda$ give in general non-isomorphic objects which differ by quadratic characters of $\operatorname{Gal}\left(\mathbf{k}^{s e p} / \mathbf{k}\right)$. 


\subsection{Cohomological Hall algebra in the case of a smooth algebra with potential}

Let $R$ be a smooth $I$-bigraded algebra over field $\mathbf{k}$ endowed with a bilinear form $\chi_{R}$ on $\mathbb{Z}^{I}$ compatible with Euler characteristic as in Section 3.3.

Let us assume that we are given an element

$$
W \in R /[R, R]
$$

represented by some element $\widetilde{W} \in R, W=\widetilde{W}(\bmod [R, R])$. The element $W$ (or its lifting $\widetilde{W}$ ) is called a potential ${ }^{8}$. Then for any $\gamma \in \mathbb{Z}_{\geqslant 0}^{I}$ we obtain a function $W_{\gamma}$ on the affine variety $\mathrm{M}_{\gamma}$, invariant under the action of $\mathrm{G}_{\gamma}$. The value of $W_{\gamma}$ at any representation is given by the trace of the image of $\widetilde{W}$. For any short exact sequence

$$
0 \rightarrow E_{1} \hookrightarrow E \rightarrow E_{2} \rightarrow 0
$$

of representations of $R$ we have $W_{\gamma_{1}+\gamma_{2}}(E)=W_{\gamma_{1}}\left(E_{1}\right)+W_{\gamma_{2}}\left(E_{2}\right)$, where $\gamma_{i}$, $i=1,2$ are dimension vectors of $E_{i}, i=1,2$.

Let us denote by $\mathbf{H}$ one of the cohomology theories considered above (i.e. EMHS, Betti, de Rham or étale cohomology), with values in an appropriate Tannakian category $\mathcal{C}$.

Definition 4.9. The Cohomological Hall algebra of $(R, W)$ (in realization $\mathbf{H})$ is an associative twisted graded algebra in $\mathcal{C}$ defined by the formula

$$
\mathcal{H}:=\oplus_{\gamma} \mathcal{H}_{\gamma}, \quad \mathcal{H}_{\gamma}:=\mathbf{H}^{\bullet}\left(\mathrm{M}_{\gamma} / \mathrm{G}_{\gamma}, W_{\gamma}\right):=\mathbf{H}^{\bullet}\left(\mathrm{M}_{\gamma}^{\text {univ }}, W_{\gamma}^{\text {univ }}\right) \quad \forall \gamma \in \mathbb{Z}_{\geqslant 0}^{I}
$$

in the obvious notation. (The definition of the product and the proof of associativity are completely similar to the case of an algebra without potential).

More precisely, all the morphisms (the Künneth isomorphism, the pullback and the Gysin map) which appear in the definition of the product in Section 2.2 induce morphisms in realization $\mathbf{H}$. In the case $\mathbf{k} \subset \mathbb{C}$ the commutativity of nine small diagrams in Section 2.3 follows from the corresponding commutativity in Betti realization and from the comparison isomorphism. Alternatively, one can use the formalism of six functors for general $\mathbf{k}$.

\footnotetext{
${ }^{8}$ We also use sometimes the same word "potential" for a function on an algebraic variety when we consider exponential Hodge structures. We hope it will not lead to a confusion.
} 
As an example, we describe the algebra $\mathcal{H}$ in the case of the quiver $Q=$ $Q_{1}$ with one vertex and one loop $l$, and potential $W=\sum_{i=0}^{N} c_{i} l^{i}, c_{N} \neq 0$, given by a polynomial of degree $N \in \mathbb{Z}_{\geqslant 0}$ in one variable. Dimension vector $\gamma$ for such $Q$ is given by an integer $n \geqslant 0$. For simplicity, we consider Betti realization.

In the case $N=0$, the question reduces to $Q$ without potential, and was considered above. Algebra $\mathcal{H}$ is the polynomial algebra of infinitely many variables (see Section 2.5).

In the case $N=1$ the cohomology of pair vanishes for matrices of size greater than 0 , hence $\mathcal{H}=\mathcal{H}_{0}=\mathbb{Z}$.

In the case $N=2$ we may assume without loss of generality that $W=-l^{2}$. It is easy to see that the cohomology of

$$
\left(\operatorname{Mat}(n \times n, \mathbb{C}), \quad x \mapsto-\operatorname{Tr}\left(x^{2}\right)\right)
$$

can be identified under the restriction map with the cohomology

$$
\left(\operatorname{Herm}(n), \quad x \mapsto-\operatorname{Tr}\left(x^{2}\right)\right)
$$

where $\operatorname{Herm}(n)$ is the space of Hermitean matrices. The latter cohomology group is same as $H^{*}\left(\mathrm{D}^{n^{2}}, \partial \mathrm{D}^{n^{2}}\right)$, where $\mathrm{D}^{n^{2}}$ is the standard closed unit ball in $\mathbb{R}^{n^{2}} \simeq \operatorname{Herm}(n)$. Hence $H^{\bullet}\left(\operatorname{Mat}(n \times n, \mathbb{C}), x \mapsto-\operatorname{Tr}\left(x^{2}\right)\right)$ is isomorphic to $\mathbb{Z}$ concentrated in the cohomological degree $n^{2}$. Moreover, one can use the unitary group $U(n)$ instead of the homotopy equivalent group $\operatorname{GL}(n, \mathbb{C})$ in the definition of equivariant cohomology. Group $U(n)$ acts on the pair $\left(\mathrm{D}^{n^{2}}, \partial \mathrm{D}^{n^{2}}\right)$, and Thom isomorphism gives a canonical isomorphism of cohomology groups

$$
\mathcal{H}_{n} \simeq H^{\bullet}(\operatorname{BGL}(n, \mathbb{C}))\left[-n^{2}\right] \simeq H_{U(n)}^{\bullet}\left(\mathrm{D}^{n^{2}}, \partial \mathrm{D}^{n^{2}}\right)
$$

Let us endow $\mathcal{H}$ with the natural bigrading which was used in the case of a symmetric incidence matrix in Section 2.6. Then an easy calculation shows that $\mathcal{H}$ coincides as a bigraded abelian group with the algebra associated with the quiver $Q_{0}$ with one vertex and zero arrows. Furthermore, comparing Grassmannians which appear in the definition of the multiplication for the quiver $Q_{0}$ (see Section 2.2), with those which arise for $Q_{1}$ with potential $W=-l^{2}$, one can check that the multiplications coincide as well. Hence the algebra $\mathcal{H}=\mathcal{H}_{(Q, W)}$ is the exterior algebra with infinitely many generators.

In the case of degree $N \geqslant 3$, one can show that the bigraded algebra $\mathcal{H}$ is isomorphic to the $(N-1)$-st tensor power of the exterior algebra corresponding to the case $N=2$. The proof will be given in Section 7 , using so-called 
critical cohomology theory. In this particular case, the critical cohomology coincides with the rapid decay one.

Let us assume that for the Tannakian category $\mathcal{C}$ (target of the cohomology functor) we have the notion of weight filtration. We will say that COHA $\mathcal{H}$ is pure if for any $\gamma$ the graded space $\mathbf{H}^{\bullet}\left(\mathrm{M}_{\gamma}^{\text {univ }}, W_{\gamma}^{\text {univ }}\right)$ is pure, i.e., its $n$th component is of weight $n$ for any $n \in \mathbb{Z}$. In order to prove that $\mathcal{H}$ is pure it is sufficient to check that $\mathbf{H}^{\bullet}\left(\mathrm{M}_{\gamma}, W_{\gamma}\right)$ is pure. Indeed, in this case the spectral sequence

$$
\mathbf{H}^{\bullet}\left(\mathrm{M}_{\gamma}, W_{\gamma}\right) \otimes \mathbf{H}^{\bullet}\left(\mathrm{B} \mathrm{G}_{\gamma}\right) \Longrightarrow \mathbf{H}^{\bullet}\left(\mathrm{M}_{\gamma}^{\text {univ }}, W_{\gamma}^{\text {univ }}\right)
$$

collapses because $\mathbf{H}^{\bullet}\left(\mathrm{BG}_{\gamma}\right)$ is pure, and hence for every weight $n$ we have a complex supported only in degree $n$. Examples of pure COHA include all quivers with zero potentials as well as the above example of $Q_{1}$ with one vertex, one loop and an arbitrary potential. For $R=\mathbb{C}\left[t, t^{-1}\right]$ (quiver $Q_{1}$ with an invertible loop), and $W=0$, the corresponding COHA is not pure.

Remark 4.1. (a) In the case when $R$ is the path algebra of a quiver $Q$, the space of representations $\mathrm{M}_{\gamma}$ is the space of collections of matrices. Integrals of $\exp \left(W_{\gamma} / u\right)$ over appropriate non-compact cycles in $\mathrm{M}_{\gamma}$ (usually over the locus of Hermitean or unitary matrices) are exactly objects of study in the theory of matrix models in mathematical physics. Those integrals are encoded in the comparison isomorphism between Betti and de Rham realizations of $H_{\mathrm{EHMS}}^{\bullet}\left(\mathrm{M}_{\gamma} / \mathrm{G}_{\gamma}\right)$ and can be interpreted as periods of the corresponding "exponential motives".

(b) The non-zero constant $u$ parametrizing the comparison isomorphism corresponds to the string coupling constant $g_{\mathrm{s}}$. Notice that the parameter $u$ is the same for all dimension vectors $\gamma$. Moreover, we do not have neither a distinguished integration cycle nor a volume element. As a result we do not consider the "large N" (in our notation "large $|\gamma|$ ") behavior of matrix integrals.

(c) Barannikov developed (see [4]) a generalization of the notion of CalabiYau algebra in terms of BV-formalism for multi-trace functionals (i.e., products of trace functionals). It seems that in his formalism there is a distinguished volume element. The connection of his approach to our work is not clear, because multi-trace functionals do not enjoy the additivity property with respect to the direct sum of representations. 


\subsection{Example: linear potentials and cohomological dimension 2}

We calculate an exponential motive in the following general situation. Let $\pi: X \rightarrow Y$ be a fibration by affine spaces between smooth equidimensional algebraic varieties over $\mathbf{k}$ endowed with an action of an affine algebraic group $G$, and $f \in \mathcal{O}(X)^{G}$ be a function which is affine along fibers of $\pi$ (i.e., $f$ is a polynomial of degree $\leqslant 1$ on $\pi^{-1}(y)$ for $\left.\forall y\right)$. Denote by $Z \subset Y$ the closed subvariety $Z \subset Y$ of points $y \in Y$ such that $f$ is constant on $\pi^{-1}(y)$, and let $f_{Z}$ be the induced function on $Z$.

Proposition 4.5. Under the above assumptions, there is a natural isomorphism

$$
\mathbf{H}^{\bullet}(X / G, f) \simeq \mathbf{H}_{\mathrm{c}}^{\bullet}\left(Z / G,-f_{Z}\right)^{\vee} \otimes \mathbb{T}^{\otimes \operatorname{dim} Y / G},
$$

where the cohomology of a stack is defined via the universal bundle construction as in Section 1.

Proof. Notice first that

$$
\mathbf{H}^{\bullet}\left(X-\pi^{-1}(Z), f\right) \simeq 0
$$

because $f_{*}\left(\mathbb{Q}_{X-\pi^{-1}(Z)}\right)$ is a constant sheaf on $\mathbb{A}_{\mathbf{k}}^{1}$, and the projector $\Pi$ used in the definition of exponential cohomology in any realization kills constant sheaves. The same is true for equivariant cohomology. Applying the duality we see that $\mathbf{H}_{\mathrm{c}}^{\bullet}\left(\left(X-\pi^{-1}(Z)\right) / G,-f\right)$ vanishes. Hence, (from the long exact sequence of pair $\left.\left(X, X-\pi^{-1}(Z)\right)\right)$ we see that the direct image induces an isomorphism

$$
\mathbf{H}_{\mathrm{c}}^{\bullet}\left(\pi^{-1} Z / G,-f_{\mid \pi^{-1}(Z)}\right) \simeq \mathbf{H}_{\mathrm{c}}^{\bullet}(X / G,-f) .
$$

Applying Poincaré duality for $X / G$ and Thom isomorphism for the affine fibration $\pi^{-1}(Z) \rightarrow Z$, we obtain the statement of the Proposition.

Let $R_{0}$ be an $I$-bigraded smooth algebra and $N$ be a finitely generated projective $R_{0}$-bimodule. Then the free algebra $R$ generated by $N$ over $R_{0}$,

$$
R=R_{0} \oplus N \oplus\left(N \otimes_{R_{0}} N\right) \oplus \ldots
$$

is again an $I$-bigraded smooth algebra. We assume that both Euler forms for $R$ and $R_{0}$ are represented as pullbacks of forms $\chi_{R}$ and $\chi_{R_{0}}$ on $\mathbb{Z}^{I}$. For example, $R_{0}$ can be the path algebra of a quiver $Q_{0}$ with vertex set $I$, and 
$R$ can be the path algebra of a larger quiver $Q \supset Q_{0}$ with the same vertex set.

Let $W=W^{(0)}+W^{(1)} \in R /[R, R]$ be a potential which comes from an element $\widetilde{W}=\widetilde{W}^{(0)}+\widetilde{W}^{(1)} \in R_{0} \oplus N \subset R$. Then for any dimension vector $\gamma \in \mathbb{Z}_{\geqslant 0}^{I}$ the representation space $\mathrm{M}_{\gamma}$ for $R$ is a vector bundle over the space $\mathrm{M}_{\gamma}^{0}$ parametrizing $\gamma$-dimensional representations of $R_{0}$, and the potential $W_{\gamma}$ is affine along fibers. Hence, we can apply the above Proposition.

We obtain

$$
\mathcal{H}_{\gamma} \simeq \mathbf{H}_{\mathrm{c}}^{\bullet}\left(\mathrm{M}_{\gamma}^{1} / \mathrm{G}_{\gamma},-W_{\gamma}^{(0)}\right)^{\vee} \otimes \mathbb{T}^{-\otimes \chi_{R_{0}}(\gamma, \gamma)}
$$

where $\mathrm{M}_{\gamma}^{1} \subset \mathrm{M}_{\gamma}^{0}$ is a closed $\mathrm{G}_{\gamma}$-invariant subvariety parametrizing fibers of fibration $\mathrm{M}_{\gamma} \rightarrow \mathrm{M}_{\gamma}^{0}$ along which $\widetilde{W}_{\gamma}^{(1)}$ vanishes. It is easy to see that $\mathrm{M}_{\gamma}^{1}$ is the variety of representations of a certain quotient algebra $R_{1}=R_{0} / J$ of $R_{0}$. The two-sided ideal $J$ is the image of the evaluation morphism

$$
\operatorname{Hom}_{R_{0}-\bmod -R_{0}}\left(N, R_{0}\right) \rightarrow R_{0}, \quad \phi \mapsto \phi\left(\widetilde{W}^{(1)}\right)
$$

For example, if $R_{0}$ is path algebra of a quiver $Q_{0}$ with vertex set $I$, a larger quiver $Q$ is obtained from $Q_{0}$ by adding finitely many edges, and the potential $W^{(1)}$ is a cyclic polynomial in arrows of $Q$, which is linear in new edges, then $R_{1}$ is the quotient of $R_{0}$ by the relations $\partial W^{(1)} / \partial \alpha=0$ for all $\alpha \in \operatorname{Edges}(Q)-\operatorname{Edges}\left(Q_{0}\right)$. In particular, any finitely presented $I$-bigraded algebra can be obtained in such a way as $R_{1}=R_{0} / J$.

Algebra $R_{1}$ is isomorphic to $H^{0}\left(R_{1}^{\prime}\right)$, where $R_{1}^{\prime}$ is a differential graded algebra concentrated in non-positive degrees. Forgetting the differential we see that the algebra $R_{1}^{\prime}$ is smooth. It is generated (over the algebra $R_{0}$ placed in degree 0$)$ by the bimodule $M:=\operatorname{Hom}_{R_{0}-\bmod -R_{0}}\left(N, R_{0} \otimes R_{0}\right)$ placed in degree -1 . Element $\widetilde{W}$ gives a differential $d: \alpha \mapsto \operatorname{mult} \circ \alpha(\widetilde{W}), \alpha \in M$, where mult : $R_{0} \otimes R_{0} \rightarrow R_{0}$ is the product. Clearly $d(\alpha)=0$ for $\alpha \in R_{0}$. If $R_{1}$ is quasi-isomorphic to $R_{1}^{\prime}$ then $R_{1}$ has global cohomological dimension $\leqslant 2$. Examples include preprojective algebras and algebras of functions on smooth affine surfaces. In general, the abelian category of $R_{1}$-modules admits a natural dg-enhancement such that for any two objects one has Ext-groups only in degrees $0,1,2$.

Remark 4.2. This construction indicates that it should be possible to define Cohomological Hall algebra for a larger class of abelian categories than $R$-mod, e.g., for the category of coherent sheaves on any smooth projective surface. For such categories moduli stacks of objects are not smooth (and 
not of finite type), but have virtual fundamental classes (as functionals on the cohomology with compact support) as well as virtual dimensions.

\section{Stability conditions, wall-crossing and motivic DT-series}

In this section, we introduce the generating series which is an analog of the motivic Donaldson-Thomas series from [33]. It is defined in terms of COHA of a smooth $I$-bigraded algebra with potential. A choice of stability function gives rise to a "PBW decomposition" of the generating series with factors parametrized by different rays in the upper-half plane. We also study how the series changes under a mutation of quiver with potential.

\subsection{Stability and Harder-Narasimhan filtration}

Let us fix a smooth $I$-bigraded algebra $R$ over $\mathbf{k}$ (possibly endowed with a potential, or even with a closed 1-form), for a given finite set $I$. Also we fix an algebraic closure $\overline{\mathbf{k}}$ of the ground field $\mathbf{k}$. We have two abelian categories: $\mathfrak{A}$ which is k-linear and whose objects are k-points of schemes $\mathbf{M}_{\gamma}$, and its $\overline{\mathbf{k}}$-linear cousin $\mathfrak{A}_{\overline{\mathbf{k}}}$ which is $\overline{\mathbf{k}}$-linear and whose objects are $\overline{\mathbf{k}}$-points of $\mathbf{M}_{\gamma}$. We will work mostly with $\mathfrak{A}_{\overline{\mathbf{k}}}$.

Definition 5.1. For given $I$, a central charge $Z$ (a.k.a. stability function) is an additive map $Z: \mathbb{Z}^{I} \rightarrow \mathbb{C}$ such that the image of any of the standard base vectors lies in the upper-half plane $\mathbb{H}_{+}:=\{z \in \mathbb{C} \mid \operatorname{Im}(z)>0\}$. Central charge $Z$ is called 'generic' if there are no two $\mathbb{Q}$-independent elements of $\mathbb{Z}_{\geqslant 0}^{I}$, which are mapped by $Z$ to the same straight line.

Let us fix a central charge $Z$. Then for any non-zero object $0 \neq E \in$ $\operatorname{Ob}\left(\mathfrak{A}_{\overline{\mathbf{k}}}\right)$ one defines

$$
\operatorname{Arg}(E):=\operatorname{Arg}(Z(c l(E))) \in(0, \pi)
$$

where $\gamma=\operatorname{cl}(E) \in \mathbb{Z}_{\geqslant 0}^{I}$ is the dimension vector of the object $E$, i.e., $E \in$ $\mathrm{M}_{\gamma}(\overline{\mathbf{k}})$. We will also use the shorthand notation $Z(E):=Z(c l(E))$.

Definition 5.2. A non-zero object $E$ is called semistable (for the central charge $Z$ ) if there is no non-zero subobject $F \subset E$ such that $\operatorname{Arg}(F)>$ $\operatorname{Arg}(E)$. 
It is easy to see that the set of semistable objects is the set $\overline{\mathbf{k}}$-points of a Zariski open $\mathrm{G}_{\gamma}$-invariant subset $\mathrm{M}_{\gamma}^{s s} \subset \mathrm{M}_{\gamma}(\overline{\mathbf{k}})$ defined over $\mathbf{k}$. In particular it is smooth.

Any non-zero object $E$ of $\mathfrak{A}_{\overline{\mathbf{k}}}$ admits a canonical Harder-Narasimhan filtration (HN-filtration in short), i.e., an increasing filtration $0=E_{0} \subset E_{1} \subset$ $\cdots \subset E_{n}=E$ with $n \geqslant 1$ such that all the quotients $F_{i}:=E_{i} / E_{i-1}, i=$ $1, \ldots, n$ are semistable and

$$
\operatorname{Arg}\left(F_{1}\right)>\cdots>\operatorname{Arg}\left(F_{n}\right)
$$

It will be convenient to introduce a total order $\prec$ (a lexicographic order in polar coordinates) on the upper-half plane by

$$
z_{1} \prec z_{2} \text { iff } \operatorname{Arg}\left(z_{1}\right)>\operatorname{Arg}\left(z_{2}\right) \text { or }\left\{\operatorname{Arg}\left(z_{1}\right)=\operatorname{Arg}\left(z_{2}\right) \text { and }\left|z_{1}\right|>\left|z_{2}\right|\right\} \text {. }
$$

One can show easily that if $0=E_{0} \subset E_{1} \subset \ldots$ is a HN-filtration of a nonzero object $E$, then $E$ does not contain a subobject $F \subset E$ with

$$
Z(F) \prec Z\left(E_{1}\right)
$$

and the unique non-zero subobject $F^{\prime}$ of $E$ with $Z\left(F^{\prime}\right)=Z\left(E_{1}\right)$ is $F^{\prime}=E_{1}$.

For a non-zero $\gamma \in \mathbb{Z}_{\geqslant 0}^{I}$ let us denote by $\mathcal{P}(\gamma)$ the set of collections

$$
\gamma_{\bullet}=\left(\gamma_{1}, \ldots, \gamma_{n}\right), n \geqslant 1, \text { such that } \sum_{i=1}^{n} \gamma_{i}=\gamma
$$

where $\gamma_{i}, i=1, \ldots, n$ are non-zero elements of $\mathbb{Z}_{\geqslant 0}^{I}$ satisfying

$$
\operatorname{Arg}\left(Z\left(\gamma_{1}\right)\right)>\operatorname{Arg}\left(Z\left(\gamma_{2}\right)\right)>\cdots>\operatorname{Arg}\left(Z\left(\gamma_{n}\right)\right)
$$

We introduce a partial "left lexicographic" order on by $\mathcal{P}(\gamma)$ by the formula

$$
\begin{aligned}
& \left(\gamma_{1}^{\prime}, \ldots, \gamma_{n^{\prime}}^{\prime}\right)<\left(\gamma_{1}, \ldots, \gamma_{n}\right) \text { if } \exists i, 1 \leqslant i \leqslant \min \left(n, n^{\prime}\right) \text { such that } \\
& \gamma_{1}^{\prime}=\gamma_{1}, \ldots, \gamma_{i-1}^{\prime}=\gamma_{i-1} \text { and } Z\left(\gamma_{i}^{\prime}\right) \prec Z\left(\gamma_{i}\right) \text {. }
\end{aligned}
$$

This order is in fact a total order for generic $Z$. We denote by $\mathbf{M}_{\gamma ; \gamma_{1}, \ldots, \gamma_{n}}$ the constructible subset of $\mathrm{M}_{\gamma}$ whose $\overline{\mathbf{k}}$-points are objects $E \in \mathrm{M}_{\gamma}(\overline{\mathbf{k}})$ which 
admit an increasing filtration $0=E_{0} \subset E_{1} \subset \cdots \subset E_{n}$ such that

$$
\operatorname{cl}\left(E_{i} / E_{i-1}\right)=\gamma_{i}, \quad i=1, \ldots, n .
$$

One can see that the subset $\mathbf{M}_{\gamma ; \gamma_{1}, \ldots, \gamma_{n}}$ is closed, because $\mathbf{M}_{\gamma ; \gamma_{1}, \ldots, \gamma_{n}}$ is the image under projection to $M_{\gamma}$ of a closed subset in the product of $M_{\gamma}$ and of the appropriate flag varieties associated with the vertices of the quiver.

Lemma 5.1. For any non-zero $\gamma \in \mathbb{Z}_{\geqslant 0}^{I}$ and any $\gamma_{\bullet} \in \mathcal{P}(\gamma)$ the complement

$$
\mathrm{M}_{\gamma, \gamma_{\bullet}}-\cup_{\gamma_{\bullet}<\gamma_{\bullet}} \mathrm{M}_{\gamma, \gamma_{\bullet}^{\prime}}
$$

coincides with the constructible subset $\mathrm{M}_{\gamma, \gamma_{\bullet}}^{\mathrm{HN}} \subset \mathrm{M}_{\gamma}$ consisting of representations whose $H N$-filtration has type $\gamma_{\bullet}$.

Proof. First, take $E \in \mathrm{M}_{\gamma, \gamma_{\bullet}}^{\mathrm{HN}}$ and assume that $E \in \mathrm{M}_{\gamma, \gamma_{\bullet}^{\prime}}$ for some $\gamma_{\bullet}^{\prime}<\gamma_{\bullet}$. Hence we have two filtrations on $E$, the HN-filtration $E_{\bullet}^{\mathrm{HN}}$ and a filtration $E_{\bullet}^{\prime}$ with quotients with dimension vectors $\gamma_{\bullet}^{\prime}$. Denote by $i$ the minimal index for which $\gamma_{i} \neq \gamma_{i}^{\prime}$. It is easy to show by induction that both filtrations coincide up to index $i-1$, and that the inequality $Z\left(\gamma_{i}^{\prime}\right) \prec Z\left(\gamma_{i}\right)$ is not compatible with the stability of the quotient $E_{i}^{\mathrm{HN}} / E_{i-1}^{\mathrm{HN}}$. Hence our assumption cannot be satisfied.

Conversely, let us assume that $E \in \mathrm{M}_{\gamma, \gamma_{\bullet}}$ does not belong to $\mathrm{M}_{\gamma, \gamma_{\bullet}}^{\mathrm{HN}}$. Denote by $E_{\bullet}^{\mathrm{HN}}$ the HN-filtration for $E$, and by $E_{\bullet}$ any filtration with dimensions of quotients given by the vector $\gamma_{\bullet}$. Let us denote by $i$ the minimal index for which $\gamma_{i}^{\mathrm{HN}} \neq \gamma_{i}$. We have as above $E_{j}=E_{j}^{\mathrm{HN}}$ for all $j<i$, and $E_{i} \neq E_{i}^{\mathrm{HN}}$. Then $E_{i}^{\mathrm{HN}} / E_{i-1}^{\mathrm{HN}}$ is the lowest non-zero term in the HN-filtration for $E / E_{i-1}^{\mathrm{HN}}$, and $E_{i} / E_{i-1}^{\mathrm{HN}} \neq E_{i}^{\mathrm{HN}} / E_{i-1}^{\mathrm{HN}}$. Hence we have $Z\left(\gamma_{i}^{\mathrm{HN}}\right) \prec Z\left(\gamma_{i}\right)$. Therefore, we have $\gamma_{\bullet}^{\mathrm{HN}}<\gamma_{\bullet}$, and $E$ belongs to the union $\cup_{\gamma_{\bullet}<\gamma_{\bullet}} \mathrm{M}_{\gamma, \gamma_{\bullet}^{\prime}}$.

For any non-zero $\gamma \in \mathbb{Z}_{\geqslant 0}^{I}$ and any $\gamma_{\bullet} \in \mathcal{P}(\gamma)$, we define a closed set

$$
\mathrm{M}_{\gamma, \leqslant \gamma_{\bullet}}:=\cup_{\gamma_{\bullet}^{\prime} \leqslant \gamma_{\bullet}} \mathrm{M}_{\gamma, \gamma_{\bullet}^{\prime}}
$$

Let us assume for simplicity that $Z$ is generic, hence the partial order $<$ is a total order. Above considerations imply that we get a chain of closed sets whose consecutive differences are exactly sets $\mathrm{M}_{\gamma, \gamma_{\bullet}}^{\mathrm{HN}}$. Taking the complements, we obtain a chain of open subspaces

$$
\emptyset=U_{0} \subset U_{1} \subset \cdots \subset U_{N_{\gamma}}=\mathrm{M}_{\gamma}
$$

where $N_{\gamma}:=\# \mathcal{P}(\gamma)$ is the number of elements of the finite set $\mathcal{P}(\gamma)$. The consecutive differences $U_{i}-U_{i-1}$ are exactly locally closed spaces $\mathrm{M}_{\gamma, \gamma_{\bullet}}^{\mathrm{HN}}$. 
This chain is obviously invariant under the action of $\mathrm{G}_{\gamma}$, and we obtain a chain of open subspaces

$$
U_{l}^{\text {univ }} \subset \mathrm{M}_{\gamma}^{\text {univ }}, l=0, \ldots, N_{\gamma}
$$

\subsection{Spectral sequence converging to $\mathcal{H}_{\gamma}$}

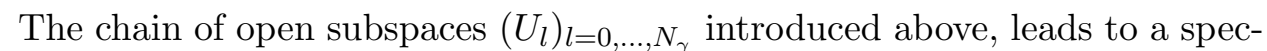
tral sequence converging to $\mathcal{H}_{\gamma}=\mathbf{H}_{\mathrm{G}_{\gamma}}^{\bullet}\left(\mathrm{M}_{\gamma}, W_{\gamma}\right)$ with the first term

$$
\bigoplus_{l=1}^{N_{\gamma}} \mathbf{H}_{\mathrm{G}_{\gamma}}^{\bullet}\left(U_{l}, U_{l-1}, W_{\gamma}\right)
$$

Theorem 5.1. In the above notation, the first term is isomorphic to

$$
\bigoplus_{n \geqslant 0} \bigoplus_{\substack{\gamma_{1}, \ldots, \gamma_{n} \in \mathbb{Z}_{\geqslant 0}^{I}-0 \\ \operatorname{Arg} \gamma_{1}>\cdots>\operatorname{Arg} \gamma_{n}}} \mathbf{H}_{\prod_{i \in I}}^{\bullet \mathrm{G}_{\gamma_{i}}}\left(\mathrm{M}_{\gamma_{1}}^{s s} \times \cdots \times \mathrm{M}_{\gamma_{n}}^{s s}, W_{\gamma}\right) \otimes \mathbb{T}^{\otimes \sum_{i<j}\left(-\chi_{R}\left(\gamma_{i}, \gamma_{j}\right)\right)}
$$

Proof. First, for a given decomposition $\gamma_{\bullet}=\left(\gamma_{1}, \ldots, \gamma_{n}\right) \in \mathcal{P}(\gamma)$, we define $\mathrm{M}_{\gamma_{1}, \ldots, \gamma_{n}} \subset \mathrm{M}_{\gamma}$ as the space of representations of $R$ in coordinate spaces, such that collections of standard coordinate subspaces $\left(\overline{\mathbf{k}}^{\sum_{j \leqslant k} \gamma_{i}^{j}}\right)_{i \in I}$ form a subrepresentation of $R$ for any $k=0, \ldots, n$. This space is smooth, because it is the scheme of representations of $R$ in a finite-dimensional algebra (the product over $i \in I$ of algebras of block upper-triangular matrices). Denote by $\mathrm{M}_{\gamma_{1}, \ldots, \gamma_{n}}^{s s} \subset \mathrm{M}_{\gamma_{1}, \ldots, \gamma_{n}}$ the open subspace consisting of representations such that the associated subquotients of dimension vectors $\gamma_{i}, i=1, \ldots, n$ are semistable. In particular, $\mathbf{M}_{\gamma_{1}, \ldots, \gamma_{n}}^{s s}$ is smooth.

It follows from definitions that

$$
\mathrm{M}_{\gamma, \gamma_{\bullet}}^{\mathrm{HN}} \simeq \mathrm{G}_{\gamma} \times_{\mathrm{G}_{\gamma_{1}, \ldots, \gamma_{n}}} \mathrm{M}_{\gamma_{1}, \ldots, \gamma_{n}}^{s s}
$$

where $\mathrm{G}_{\gamma_{1}, \ldots, \gamma_{n}} \subset \mathrm{G}_{\gamma}$ is the group of invertible block upper-triangular matrices, acting naturally on $\mathrm{M}_{\gamma_{1}, \ldots, \gamma_{n}}$ and on $\mathrm{M}_{\gamma_{1}, \ldots, \gamma_{n}}^{s s}$. This formula shows that $\mathrm{M}_{\gamma, \gamma_{\bullet}}^{\mathrm{HN}}$ is a smooth manifold. Its codimension in $\mathrm{M}_{\gamma}$ can be easily calculated:

$$
\operatorname{codim} \mathrm{M}_{\gamma, \gamma_{\bullet}}^{\mathrm{HN}}=-\sum_{i<j} \chi_{R}\left(\gamma_{i}, \gamma_{j}\right)
$$


For given $\gamma_{\bullet} \in \mathcal{P}(\gamma)$, the component of the first term of the spectral sequence, corresponding to $\mathrm{M}_{\gamma, \gamma_{\bullet}}^{\mathrm{HN}}$, has the form

$$
\mathbf{H}_{\mathrm{G}_{\gamma}}^{\bullet}\left(U_{l}, U_{l-1}, W_{\gamma}\right)
$$

for some $l, 1 \leqslant l \leqslant N_{\gamma}$, where $\mathrm{M}_{\gamma, \gamma}^{\mathrm{HN}}$ is a closed $\mathrm{G}_{\gamma}$-invariant submanifold of the manifold $U_{l}$, with the complement $U_{l}-\mathrm{M}_{\gamma, \gamma_{\bullet}}^{\mathrm{HN}}$ equal to $U_{l-1}$. Hence, by Thom isomorphism we see that this component can be written as

$$
\mathbf{H}_{\mathrm{G}_{\gamma}}^{\bullet}\left(\mathrm{M}_{\gamma, \gamma \bullet}^{\mathrm{HN}}, W_{\gamma}\right) \otimes \mathbb{T}^{\otimes\left(-\sum_{i<j} \chi_{R}\left(\gamma_{i}, \gamma_{j}\right)\right)} .
$$

Finally, using the isomorphism

$$
\mathbf{H}_{\mathrm{G}_{\gamma}}^{\bullet}\left(\mathrm{M}_{\gamma, \gamma_{\bullet}}^{\mathrm{HN}}, W_{\gamma}\right) \simeq \mathbf{H}_{\mathrm{G}_{\gamma_{1}, \ldots, \gamma_{n}}}^{\bullet}\left(\mathrm{M}_{\gamma_{1}, \ldots, \gamma_{n}}^{s s}, W_{\gamma}\right)
$$

and $\mathbb{A}^{1}$-homotopies

$$
\mathrm{M}_{\gamma_{1}}^{s s} \times \cdots \times \mathrm{M}_{\gamma_{n}}^{s s} \sim \mathrm{M}_{\gamma_{1}, \ldots, \gamma_{n}}^{s s}, \mathrm{G}_{\gamma_{1}} \times \cdots \times \mathrm{G}_{\gamma_{n}} \sim \mathrm{G}_{\gamma_{1}, \ldots, \gamma_{n}}
$$

we obtain the statement of the theorem.

Alternatively, one can use cohomology with compact support of smooth Artin stacks $\mathrm{M}_{\gamma, \gamma_{\bullet}}^{\mathrm{HN}} / \mathrm{G}_{\gamma}$ with potential $W_{\gamma}$; see Section 5.4.

Let $V \subset \mathbb{H}_{+}$be a sector, i.e., $V=V+V=\mathbb{R}_{>0} \cdot V$ and $0 \notin V$. Denote by $\mathrm{M}_{V, \gamma} \subset \mathrm{M}_{\gamma}$ the set of all representations whose HN-factors have classes in $Z^{-1}(V)$. It is easy to see that for any $\gamma$ the set $\mathrm{M}_{V, \gamma}$ is Zariski open and $\mathrm{G}_{\gamma}$-invariant. We define the Cohomological Hall vector space by

$$
\mathcal{H}_{V}:=\oplus_{\gamma} \mathcal{H}_{V, \gamma}=\oplus_{\gamma} \mathbf{H}_{\mathrm{G}_{\gamma}}^{\bullet}\left(\mathrm{M}_{V, \gamma}, W_{\gamma}\right)
$$

Similarly to Theorem 5.1, one has a spectral sequence converging to $\mathcal{H}_{V}$ where we use only rays lying in $V$. In general the space $\mathcal{H}_{V}$ does not carry a product, because the corresponding Grassmannians are not necessarily closed. Nevertheless, for $V$ being a ray $l=\exp (\mathrm{i} \phi) \cdot \mathbb{R}_{>0}$ (as well as for $V$ equal to the whole upper-half plane), the product is well defined (and is twisted associative) because the corresponding Grassmannians are closed.

If we assume that the restriction of the bilinear form $\chi_{R}$ to the sublattice $Z^{-1}(\exp (\mathrm{i} \phi) \cdot \mathbb{R})$ is symmetric (e.g., it always holds for generic $\left.Z\right)$, then we 
have the modified untwisted associative product on

$$
\mathcal{H}_{l}^{\bmod }:=\oplus_{\gamma \in Z^{-1}(l)} \mathcal{H}_{l, \gamma} \otimes\left(\mathbb{T}^{\otimes 1 / 2}\right)^{\otimes \chi_{R}(\gamma, \gamma)}, l:=\exp (\mathrm{i} \phi) \cdot \mathbb{R}_{>0}
$$

The decomposition in Theorem 5.1 means that the twisted algebra $\mathcal{H}=$ $\mathcal{H}_{\mathbb{H}_{+}}$"looks like" (i.e., related by a spectral sequence with) a clockwise ordered product of twisted algebras $\mathcal{H}_{l}$ over the set of rays $l$. This is similar to a decomposition for universal enveloping algebras which follows from the Poincaré-Birkhoff-Witt theorem. Namely, if $\mathfrak{g}=\bigoplus_{\gamma \in \mathbb{Z}_{\geqslant 0}^{I}-\{0\}} \mathfrak{g}_{\gamma}$ is a graded Lie algebra (over any field), then for any ray $l \subset \mathbb{H}_{+}$we have Lie subalgebra $\mathfrak{g}_{l}:=\bigoplus_{\gamma \in Z^{-1}(l)} \mathfrak{g}_{\gamma}$, and

$$
\bigotimes_{l}^{\curvearrowright} U \mathfrak{g}_{l} \stackrel{\sim}{\longrightarrow} U \mathfrak{g}
$$

In the case of COHA the twisted algebras $\mathcal{H}_{l}$ are not naturally realized as twisted subalgebras of $\mathcal{H}$, there is only a homomorphism of graded spaces $\mathcal{H} \rightarrow \mathcal{H}_{l}$ given by the restriction morphism for the inclusions $\mathrm{M}_{\gamma}^{s s} \hookrightarrow \mathrm{M}_{\gamma}$.

In the special case of quiver $A_{2}$ and Betti cohomology, there are embeddings of algebras $\mathcal{H}_{l} \hookrightarrow \mathcal{H}$ (with cohomological $\mathbb{Z} / 2 \mathbb{Z}$-grading), as follows from direct calculations (see Section 2.8), and the clockwise ordered product indeed induces an isomorphism

$$
\bigotimes_{l}^{\curvearrowright} \mathcal{H}_{l} \stackrel{\sim}{\longrightarrow} \mathcal{H}
$$

Finally, we should warn the reader of a possible caveat. Namely, the calculation from section 4.8 is not applicable to $\mathcal{H}_{V, \gamma}$ for general $V$ because corresponding spaces $\mathrm{M}_{V, \gamma}$ are only Zariski open subsets, and do not form themselves fibrations by affine spaces. Therefore, the stability in the ambient category of cohomological dimension 1 is not directly related with the stability in the smaller category of cohomological dimension 2.

\subsection{Generating series and quantum tori}

The cohomology theory $\mathbf{H}^{\bullet}$ used in the definition of Cohomological Hall algebra takes values in a Tannakian category $\mathcal{C}$. In other words, cohomology carries an action of a pro-affine algebraic group, which we call the motivic Galois group $\mathrm{Gal}_{\mathbf{H}}^{\mathrm{mot}}$ for the theory $\mathbf{H}$. We assume that there is a notion of weight filtration in $\mathcal{C}$. For example, in the case of rapid decay Betti (tensored by $\mathbb{Q}$ ) or de Rham cohomology we consider $\mathbf{H}^{\bullet}(X)$ for any variety 
$X$ as a vector space graded by cohomological degree and endowed with the weight filtration. In terms of $\mathrm{Gal}_{\mathbf{H}}^{\text {mot }}$ this means that we have an embedding $w: \mathbb{G}_{m} \hookrightarrow \mathrm{Gal}_{\mathbf{H}}^{\text {mot }}$ (defined up to conjugation) such that the Lie algebra of $\mathrm{Gal}_{\mathbf{H}}^{\text {mot }}$ has non-positive weights with respect to the adjoint $\mathbb{G}_{m}$-action. For any representation $E$ of $\mathrm{Gal}_{\mathbf{H}}^{\text {mot }}$ the weight filtration is defined by

$$
W_{i} E:=\oplus_{j \leqslant i} E_{j}, \quad i, j \in \mathbb{Z},
$$

where $E_{j} \subset E$ is the eigenspace of $w\left(\mathbb{G}_{m}\right)$ with weight $j$.

Let us consider the $K_{0}$-ring $\mathcal{M}$ of tensor category $\mathcal{C}$ (i.e., of the category of finite-dimensional representations of $\mathrm{Gal}_{\mathbf{H}}^{\text {mot }}$ ). It contains an invertible element $\mathbb{L}$ corresponding to the Tate motive $\mathbf{H}^{2}\left(\mathbb{P}^{1}\right)$ of weight +2 . We complete $\mathcal{M}$ by adding infinite sums of pure motives with weights approaching to $+\infty$. Notice that this completion (which we denote by $\widehat{\mathcal{M}}$ ) differs from the completion used in the theory of motivic integration, where weights are allowed to go to $-\infty$.

With any $I$-bigraded smooth algebra $R$ and a bilinear form $\chi_{R}$ compatible with the Euler form, we associate the following series with coefficients in $\widehat{\mathcal{M}}$ :

$$
A=A^{(R, W)}:=\sum_{\gamma \in \mathbb{Z}_{\geqslant 0}^{I}}\left[\mathcal{H}_{\gamma}\right] \mathbf{e}_{\gamma},
$$

where variables $\mathbf{e}_{\gamma}$ are additive generators of the associative unital algebra $\mathcal{R}_{+}$over $\mathcal{M}$ isomorphic to the subalgebra of the motivic quantum torus $\mathcal{R}$ introduced in [33]. The relations are given by the formulas

$$
\mathbf{e}_{\gamma_{1}} \cdot \mathbf{e}_{\gamma_{2}}=\mathbb{L}^{-\chi_{R}\left(\gamma_{1}, \gamma_{2}\right)} \mathbf{e}_{\gamma_{1}+\gamma_{2}} \quad \forall \gamma_{1}, \gamma_{2} \in \mathbb{Z}_{\geqslant 0}^{I}, \quad \mathbf{e}_{0}=1,
$$

and the coefficient of the series $A$ is given by

$$
\left[\mathcal{H}_{\gamma}\right]:=\sum_{k \geqslant 0}(-1)^{k}\left[\mathbf{H}_{\mathrm{G}_{\gamma}}^{k}\left(\mathrm{M}_{\gamma}, W_{\gamma}\right)\right] \in \widehat{\mathcal{M}}
$$

Series $A$ belongs to the completion $\widehat{\mathcal{R}}_{+}$consisting of infinite series in $\mathbf{e}_{\gamma}$. It has the form

$$
A=1+\text { higher order terms }
$$

and is therefore invertible.

Definition 5.3. We call $A$ the motivic Donaldson-Thomas series of the pair $(R, W)$. 
The series $A$ can be written in a more explicit form using the following result.

Lemma 5.2. For any scheme $X$ of finite type endowed with an action of the affine algebraic group $G=\prod_{i} \mathrm{GL}\left(n_{i}\right)$ and a function $f \in \mathcal{O}(X)^{G}$, one has the following identity in $\widehat{\mathcal{M}}$ :

$$
\left[\mathbf{H}_{G}^{\bullet}(X, f)\right]=\left[\mathbf{H}^{\bullet}(X, f)\right] \cdot\left[\mathbf{H}^{\bullet}(\mathrm{B} G)\right]=\frac{\left[\mathbf{H}^{\bullet}(X, f)\right]}{\prod_{i}(1-\mathbb{L}) \ldots\left(1-\mathbb{L}^{n_{i}}\right)}
$$

Proof. There is a filtration of (a finite approximations of) the classifying space BG by open subschemes such that the universal $G$-bundle is trivial in consecutive differences. The spectral sequence associated with such a filtration gives the result.

In the basic example of the quiver $Q_{0}=A_{1}$ with one vertex and no edges, the motivic DT-series is

$$
\begin{aligned}
A & =1+\frac{1}{1-\mathbb{L}} \mathbf{e}_{1}+\frac{1}{(1-\mathbb{L})\left(1-\mathbb{L}^{2}\right)} \mathbf{e}_{2}+\ldots \\
& =\sum_{n \geqslant 0} \frac{\mathbb{L}^{n(n-1) / 2}}{(1-\mathbb{L}) \ldots\left(1-\mathbb{L}^{n}\right)} \mathbf{e}_{1}^{n}=\left(-\mathbf{e}_{1} ; \mathbb{L}\right)_{\infty}=\prod_{n \geqslant 0}\left(1+\mathbb{L}^{n} \mathbf{e}_{1}\right) .
\end{aligned}
$$

Let $Z: \mathbb{Z}^{I} \rightarrow \mathbb{C}$ be a central charge. Then for any sector $V \subset \mathbb{H}_{+}$we define the motivic DT-series associated with $V$ such as follows:

$$
A_{V}:=\sum_{\gamma \in \mathbb{Z}_{\geqslant 0}^{I}}\left[\mathcal{H}_{V, \gamma}\right] \mathbf{e}_{\gamma}
$$

The spectral sequence from Section 5.2 implies the following Factorization Formula:

$$
A=A_{\mathbb{H}_{+}}=\prod_{l}^{\curvearrowright} A_{l}
$$

where the product is taken in the clockwise order over all rays $l \subset \mathbb{H}_{+}$ containing non-zero points in $Z\left(\mathbb{Z}_{\geqslant 0}^{I}\right)$ (i.e., in the decreasing order of the arguments of complex numbers). Each factor $A_{l}$ corresponds to semistable 
objects with the central charge in $l$ :

$$
A_{l}=1+\sum_{\gamma \in Z^{-1}(l)} \sum_{k \geqslant 0}(-1)^{k}\left[\mathbf{H}_{\mathrm{G}_{\gamma}}^{k}\left(\mathrm{M}_{\gamma}^{s s}, W_{\gamma}\right)\right] \mathbf{e}_{\gamma} \in \widehat{\mathcal{R}}_{+}
$$

Also, for any pair of disjoint sectors $V_{1}, V_{2} \subset \mathbb{H}_{+}$whose union is also a sector, and such that $V_{1}$ lies on the left of $V_{2}$, we have

$$
A_{V_{1} \cup V_{2}}=A_{V_{1}} A_{V_{2}}
$$

It will be convenient also to rewrite series $A$ in rescaled variables. Let us add an element $\mathbb{L}^{1 / 2}$ to $\widehat{\mathcal{M}}$, satisfying the relation $\left(\mathbb{L}^{1 / 2}\right)^{2}=\mathbb{L}$ and define rescaled quantum variables by

$$
\widehat{\mathbf{e}}_{\gamma}:=\left(-\mathbb{L}^{1 / 2}\right)^{-\chi_{R}(\gamma, \gamma)} \mathbf{e}_{\gamma}
$$

The rescaled variables satisfy the relation

$$
\widehat{\mathbf{e}}_{\gamma_{1}} \cdot \widehat{\mathbf{e}}_{\gamma_{2}}=\left(-\mathbb{L}^{1 / 2}\right)^{-\left\langle\gamma_{1}, \gamma_{2}\right\rangle} \widehat{\mathbf{e}}_{\gamma_{1}+\gamma_{2}}
$$

where

$$
\left\langle\gamma_{1}, \gamma_{2}\right\rangle:=\chi_{R}\left(\gamma_{1}, \gamma_{2}\right)-\chi_{R}\left(\gamma_{2}, \gamma_{1}\right)
$$

is a skew-symmetric bilinear form on $\mathbb{Z}^{I}$. Then we have:

$$
\begin{aligned}
& A=\sum_{\gamma \in \mathbb{Z}_{\geqslant 0}^{I}}\left(-\mathbb{L}^{1 / 2}\right)^{\chi_{R}(\gamma, \gamma)}\left[\mathcal{H}_{\gamma}\right] \widehat{\mathbf{e}}_{\gamma}=\sum_{\gamma \in \mathbb{Z}_{\geqslant 0}^{I}} \frac{\left(-\mathbb{L}^{1 / 2}\right)^{\chi_{R}(\gamma, \gamma)}\left[\mathbf{H}^{\bullet}\left(\mathrm{M}_{\gamma}, W_{\gamma}\right)\right]}{\prod_{i}(1-\mathbb{L}) \ldots\left(1-\mathbb{L}^{\gamma^{i}}\right)} \widehat{\mathbf{e}}_{\gamma}
\end{aligned}
$$

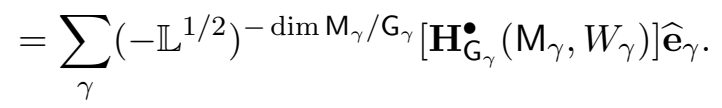

In the basic example of quiver $Q_{0}$ the motivic DT-series is $A=\left(\mathbb{L}^{1 / 2} \widehat{\mathbf{e}}_{1} ; \mathbb{L}\right)_{\infty}$.

In the case when $\chi_{R}$ is symmetric (i.e., $\langle\rangle=$,0 ), the rescaled variables are monomials in commuting variables

$$
\widehat{\mathbf{e}}_{\gamma_{1}} \cdot \widehat{\mathbf{e}}_{\gamma_{2}}=\widehat{\mathbf{e}}_{\gamma_{1}+\gamma_{2}}
$$

and the motivic DT-series $A$ is the generating series for the Serre polynomials of graded components of the associative (non-twisted) algebra $\mathcal{H}^{\text {mod }}$ with 
the modified product (see Section 2.7):

$$
A=\sum_{\gamma \in \mathbb{Z}_{\geqslant 0}^{I}}\left[\mathcal{H}_{\gamma}^{\text {mod }}\right] \widehat{\mathbf{e}}_{\gamma}
$$

In general, coefficients of the series $A$ are rational functions in the variable $\mathbb{L}^{1 / 2}$ with coefficients in $\mathcal{M}$.

There is a natural homomorphism given by the Serre polynomial

$$
S: \mathcal{M}\left[\mathbb{L}^{ \pm 1 / 2}\right] \rightarrow \mathbb{Z}\left[q^{ \pm 1 / 2}\right], S([E]):=\sum_{n \in \mathbb{Z}} \operatorname{dim} \operatorname{Gr}_{n}^{W}(E) q^{n / 2}, S\left(\mathbb{L}^{1 / 2}\right):=q^{1 / 2}
$$

where $\mathrm{Gr}_{w}^{W}$ denotes the graded component of weight $w$ with respect to the weight filtration. Applying $S$ to $A$ we obtain a series $S(A)=\sum_{\gamma} S\left(\left[\mathcal{H}_{\gamma}\right]\right) \mathbf{e}_{\gamma}$ in quantum variables with coefficients in

$$
\mathbb{Z}\left[q^{ \pm 1 / 2},\left(\left(1-q^{n}\right)^{-1}\right)_{n=1,2, \ldots}\right] \subset \mathbb{Q}\left(\left(q^{1 / 2}\right)\right)
$$

For pure COHA the series $S\left(\left[\mathcal{H}_{\gamma}\right]\right)$ for any $\gamma \in \mathbb{Z}_{\geqslant 0}^{I}$ coincides with the Hilbert-Poincaré series of the graded space $\mathcal{H}_{\gamma}$ in variable $\left(-q^{1 / 2}\right)$. Therefore for the quiver $Q_{d}$ with zero potential, we obtain the same generating series as in Section 2.5.

\subsection{Reformulation using cohomology with compact support}

It is instructive to revisit the factorization formula for the generating functions using cohomology with compact support. If we apply the involution $D: \mathcal{M} \rightarrow \mathcal{M}$ given by passing to dual motive, and extend it to the square root of Tate motive by

$$
D\left(\mathbb{L}^{1 / 2}\right)=\mathbb{L}^{-1 / 2}
$$

we obtain the series

$$
\begin{aligned}
D(A) & =\sum_{\gamma \in \mathbb{Z}_{\geqslant 0}^{I}}\left[\mathbf{H}_{c, \mathrm{G}_{\gamma}}\left(\mathrm{M}_{\gamma},-W_{\gamma}\right)\right] D\left(\mathbf{e}_{\gamma}\right)=\sum_{\gamma \in \mathbb{Z}_{\geqslant 0}^{I}} \frac{\left[\mathbf{H}_{c}\left(\mathbf{M}_{\gamma},-W_{\gamma}\right)\right]}{\left[\mathbf{H}_{c}\left(\mathbf{G}_{\gamma}\right)\right]} D\left(\mathbf{e}_{\gamma}\right) \\
& =\sum_{\gamma \in \mathbb{Z}_{\geqslant 0}^{I}} \frac{\left(-\mathbb{L}^{1 / 2}\right)^{\chi_{R}(\gamma, \gamma)}\left[\mathbf{H}_{\mathbf{c}}^{\bullet}\left(\mathrm{M}_{\gamma},-W_{\gamma}\right)\right]}{\prod_{i}\left(\mathbb{L}^{\gamma^{i}}-1\right) \ldots\left(\mathbb{L}^{\gamma^{i}}-\mathbb{L}^{i}-1\right)} D\left(\widehat{\mathbf{e}}_{\gamma}\right)
\end{aligned}
$$


where the dual quantum variables satisfy the relations:

$$
\begin{gathered}
D\left(\mathbf{e}_{\gamma_{1}}\right) \cdot D\left(\mathbf{e}_{\gamma_{2}}\right)=\mathbb{L}^{-\chi_{R}\left(\gamma_{2}, \gamma_{1}\right)} D\left(\mathbf{e}_{\gamma_{1}+\gamma_{2}}\right), \\
D\left(\widehat{\mathbf{e}}_{\gamma}\right)=\left(-\mathbb{L}^{1 / 2}\right)^{-\chi_{R}(\gamma, \gamma)} D\left(\mathbf{e}_{\gamma}\right), D\left(\widehat{\mathbf{e}}_{\gamma_{1}}\right) \cdot D\left(\widehat{\mathbf{e}}_{\gamma_{2}}\right)=\left(-\mathbb{L}^{1 / 2}\right)^{+\left\langle\gamma_{1}, \gamma_{2}\right\rangle} D\left(\widehat{\mathbf{e}}_{\gamma_{1}+\gamma_{2}}\right) .
\end{gathered}
$$

The series $D(A)$ is analogous to the generating series considered in [33] (up to the sign change $\mathbb{L}^{1 / 2} \mapsto-\mathbb{L}^{1 / 2}$ ). Namely, we can associate with $R$ an ind-constructible three-dimensional Calabi-Yau category with the $t$-structure whose heart is equivalent to the full subcategory of the category of finite-dimensional $R$-modules, consisting of critical points of $W_{\gamma}$, $\gamma \in \mathbb{Z}_{\geqslant 0}^{I}$. This category is ind-constructible, it carries a canonical orientation (in the sense of [33]), and its motivic DT-series introduced in the loc.cit. has essentially the same form as $D(A)$, but with the rapid decay cohomology replaced by the cohomology with coefficients in the sheaf of vanishing cycles. All this will be discussed in Section 7.10.

The argument with spectral sequences in Section 5.2 becomes more direct for the compactly supported cohomology. Namely, for any stratified Artin stack $\mathcal{S}$ endowed with a function $f$ we have the identity

$$
\left[\mathbf{H}_{\mathrm{c}}(\mathcal{S}, f)\right]=\sum_{\alpha}\left[\mathbf{H}_{\mathrm{c}}\left(\mathcal{S}_{\alpha}, f\right)\right],
$$

where $\left(\mathcal{S}_{\alpha}\right)$ are strata.

The calculation in Section 4.8 is also more convenient in the case of cohomology with compact support. We will illustrate it below in Section 5.6. In particular, in the case when the potential is linear in additional arrows we obtain series

$$
\sum_{\gamma}\left[\mathbf{H}_{c}\left(S_{\gamma}\right)\right] \cdot\left(-\mathbb{L}^{1 / 2}\right)^{-\operatorname{dim}_{v i r t} S_{\gamma}} D\left(\widehat{\mathbf{e}}_{\gamma}\right),
$$

where $S_{\gamma}=\mathrm{M}_{\gamma}^{1} / \mathrm{G}_{\gamma}$ is the stack of representations of the dg-algebra $R_{1}^{\prime}$ (in the notation from Section 4.8) of dimension vector $\gamma$. The virtual dimension of $S_{\gamma}$ is equal to $-\chi_{R_{1}^{\prime}}(\gamma, \gamma)$, where $\chi_{R_{1}^{\prime}}$ is a lift to $\mathbb{Z}^{I}$ of the Euler form of the dg-category of finite-dimensional $R_{1}^{\prime}$-modules.

In the case when the ground field $\mathbf{k}=\mathbb{F}_{q}$ has finite characteristic $p>0$ we can apply the multiplicative numerical invariant from Section 4.6. The resulting series is

$$
\sum_{\gamma \in \mathbb{Z}_{\geqslant 0}^{I}} \sum_{\mathcal{E} \in \mathrm{M}_{\gamma}(\mathbf{k}) / \text { iso }} \frac{\exp \left(-\frac{2 \pi i}{p} \operatorname{Trace}_{\mathbb{F}_{q} / \mathbb{F}_{p}}(W(\mathcal{E}))\right)}{|\operatorname{Aut}(\mathcal{E})|} D\left(\mathbf{e}_{\gamma}\right) .
$$


Also, the multiplication of the variables $D\left(\mathbf{e}_{\gamma}\right)$ has a very transparent form:

$$
\begin{aligned}
& \frac{1}{\left|\operatorname{Aut}\left(\mathcal{E}_{1}\right)\right|} D\left(\mathbf{e}_{\gamma_{1}}\right) \cdot \frac{1}{\left|\operatorname{Aut}\left(\mathcal{E}_{2}\right)\right|} D\left(\mathbf{e}_{\gamma_{2}}\right) \\
& =\sum_{\mathcal{E}_{1} \rightarrow \mathcal{E} \rightarrow \mathcal{E}_{2}} \frac{1}{\left|\operatorname{Aut}\left(\mathcal{E}_{1} \rightarrow \mathcal{E} \rightarrow \mathcal{E}_{2}\right)\right|} D\left(\mathbf{e}_{\gamma_{1}+\gamma_{2}}\right),
\end{aligned}
$$

where $\mathcal{E}_{1}, \mathcal{E}_{2}$ have dimension vectors $\gamma_{1}, \gamma_{2}$ respectively.

\subsection{Classical limit}

Let us add to the quantum torus additional invertible generators $\left(\mathbf{f}_{i}\right)_{i \in I}$ satisfying the relations

$$
\mathbf{f}_{i} \cdot \mathbf{f}_{j}=\mathbf{f}_{j} \cdot \mathbf{f}_{i}, \quad \mathbf{f}_{i} \cdot \mathbf{e}_{\gamma}=\mathbb{L}^{\gamma^{i}} \mathbf{e}_{\gamma} \cdot \mathbf{f}_{i} \quad \forall i, j \in I, \gamma \in \mathbb{Z}^{I}
$$

For any $i_{0} \in I$ we define an invertible series $A^{\left(i_{0}\right)}$ in variables $\mathbf{e}_{\gamma}$ by the formula

$$
A \cdot \mathbf{f}_{i_{0}} \cdot A^{-1}=A^{\left(i_{0}\right)} \cdot \mathbf{f}_{i_{0}} \Leftrightarrow A^{\left(i_{0}\right)}:=A \cdot\left(\mathbf{f}_{i_{0}} A \mathbf{f}_{i_{0}}^{-1}\right)^{-1}
$$

More generally, we define an element $A^{(\gamma)}$ for any $\gamma \in \mathbb{Z}^{I}$ such as follows:

$$
A^{(\gamma)}:=A \cdot\left(\mathbf{f}^{\gamma} A \mathbf{f}^{-\gamma}\right)^{-1}, \quad \mathbf{f}^{\gamma}:=\prod_{i \in I} \mathbf{f}_{i}^{\gamma^{i}}
$$

It is equal to a finite product of elements $A^{(i)}$ and of conjugates of these elements by monomials in $\mathbf{f}_{j}$ (or inverses of such expressions). Also one has

$$
A \cdot \mathbf{e}_{\gamma} \cdot A^{-1}=A^{\left(\gamma^{\prime}\right)} \cdot \mathbf{e}_{\gamma}, \quad\left(\gamma^{\prime}\right)^{i}:=\sum_{j} c_{i j} \gamma^{j}
$$

where $\left(c_{i j}\right)_{i, j \in I}$ is the matrix in the standard basis of the skew-symmetric form $\langle\rangle=,\chi_{R}-\left(\chi_{R}\right)^{t}$ on $\mathbb{Z}^{I}$. E.g. for the case of a quiver in notation of Section 2.1 one has

$$
c_{i j}=a_{j i}-a_{i j}
$$

Theorem 5.2. For every $\gamma \in \mathbb{Z}^{I}$ each coefficient of the series $A^{(\gamma)}$ belongs to the subring $\mathcal{M}$ of $\widehat{\mathcal{M}}$. 
Therefore, we can apply Euler characteristic and obtain a symplectomorphism $T$ of the classical symplectic double torus ${ }^{9}$ with coordinates

$$
\left(\mathbf{e}_{i}^{c l}, \mathbf{f}_{i}^{c l}\right)_{i \in I}
$$

given by the following automorphism of the algebra of functions:

$$
\mathbf{e}_{i}^{c l} \mapsto \prod_{j \in I}\left(A^{(j), c l}\right)^{c_{j i}} \mathbf{e}_{i}^{c l}, \quad \mathbf{f}_{i}^{c l} \mapsto A^{(i), c l} \mathbf{f}_{i}^{c l},
$$

where $A^{(i), c l}$ is a series with integer coefficients in variables $\left(\mathbf{e}_{j}^{c l}\right)_{j \in I}$ given by

$$
A^{(i), c l}:=\chi\left(A^{(i)}\right)
$$

Here $\chi$ denotes the Euler characteristic. Symplectomorphism $T$ is the classical limit $\lim _{q^{1 / 2} \rightarrow 1} \operatorname{Ad}(S(A))$ where $S$ denotes Serre polynomial (cf. [33]).

Proof. It is sufficient to prove that $A^{(i)}$ has coefficients in $\mathcal{M}$ for any $i \in I$. Let us fix a vertex $i=i_{0} \in I$. Let us consider a larger index set $\widetilde{I}=I \sqcup\left\{i_{0}^{*}\right\}$, where $i_{0}^{*}$ is a new vertex, and a larger algebra $\widetilde{R}$ bigraded by $\widetilde{I}$ in such a way that a representation $\widetilde{E}$ of $\widetilde{R}$ is the same as a triple $(E, V, \phi)$, where $E$ is a representation of $R$, vector space $V=E_{i_{0}^{*}}:=p_{i_{0}^{*}}(\widetilde{E})$ is associated with $i_{0}^{*}$, and $\phi$ is a map from $V$ to the space $p_{i_{0}} E$. Here $p_{i}$ denotes the projector corresponding to the vertex $i$. If $R$ is the path algebra of a quiver $Q$ with the set of vertices $I$, then $\widetilde{R}$ is the path algebra of a new quiver $\widetilde{Q}$ obtained from $Q$ by adding a new vertex $i_{0}^{*}$ and an arrow from $i_{0}^{*}$ to $i_{0}$. We extend the potential from $R$ to $\widetilde{R} \supset R$ in a straightforward way.

For the representations of the modified algebra $\widetilde{R}$ we consider two stability conditions $Z=\left(z_{i}\right)_{i \in \widetilde{I}}$ : one with $z_{i_{0}^{*}}$ very far to the left of all $\left(z_{i}\right)_{i \in I}$, and the one with $z_{i_{0}^{*}}$ on the right. For these two stability conditions we compare the coefficients of the monomials in the Factorization formula from Section 5.2 with dimension at $i_{0}^{*}$ equal to one. This leads to the following equality:

$$
\left(\sum_{\gamma \in \mathbb{Z}_{\geqslant 0}^{I}}\left[\mathbf{H}^{\bullet}\left(\mathrm{M}_{\gamma}^{\left(i_{0}\right)} / \mathrm{G}_{\gamma}, W_{\gamma}\right)\right] \mathbf{e}_{\gamma} \mathbf{e}_{i_{0}^{*}}\right) \cdot A=A \cdot \mathbf{e}_{i_{0}^{*}}
$$

\footnotetext{
${ }^{9}$ Symplectic double torus is naturally associated with the "double" lattice $\mathbb{Z}^{I} \oplus$ $\left(\mathbb{Z}^{I}\right)^{\vee}$; see $[33]$.
} 
where $\mathrm{M}_{\gamma}^{\left(i_{0}\right)}$ is the set of pairs $(E, v)$, where $E$ is a representation of $R$ with dimension vector $\gamma$ and a cyclic vector $v \in E_{i_{0}}$. Therefore, we have:

$$
A^{\left(i_{0}\right)}=\sum_{\gamma \in \mathbb{Z}_{\geqslant 0}^{I}}\left[\mathbf{H}^{\bullet}\left(\mathrm{M}_{\gamma}^{\left(i_{0}\right)} / \mathrm{G}_{\gamma}, W_{\gamma}\right)\right] \mathbf{e}_{\gamma}
$$

The gauge group $\mathrm{G}_{\gamma}$ acts freely on $\mathrm{M}_{\gamma}^{\left(i_{0}\right)}$, hence cohomology of the quotient is finite-dimensional.

All the above considerations can be generalized to the case of a stability condition and of a sector $V \subset \mathbb{H}_{+}$. Finiteness of coefficients of the series $A_{V}^{(i), c l}$ cannot be proven in the same way as above, but it can be deduced from a very powerful integrality result proven in the next section.

\subsection{Examples}

First of all, any quiver with zero potential gives a kind of $q$-hypergeometric series:

$$
A=\sum_{\gamma \in \mathbb{Z}_{\geqslant 0}^{I}}\left[\mathbf{H}^{\bullet}\left(B \mathrm{G}_{\gamma}\right)\right] \mathbf{e}_{\gamma}=\sum_{\gamma \in \mathbb{Z}_{\geqslant 0}^{I}} \frac{\left(-\mathbb{L}^{1 / 2}\right)^{\chi_{Q}(\gamma, \gamma)}}{\prod_{i}(1-\mathbb{L}) \ldots\left(1-\mathbb{L}^{\gamma^{i}}\right)} \widehat{\mathbf{e}}_{\gamma}
$$

Applying mutations from the next section, one can get further examples with non-zero potentials.

One can easily write an explicit formula for the symplectomorphism $T$ in the case of a quiver with zero potential. This is a result of Reineke (see [47]). We give a sketch of the proof for completeness.

Theorem 5.3. For a quiver $Q$ with incidence matrix $\left(a_{i j}\right)_{i, j \in I}$ and zero potential, series $A^{(i), c l}$ are algebraic functions, and they form a unique solution (in invertible formal series) of the system of polynomial equations

$$
A^{(i), c l}=1+\boldsymbol{e}_{i}^{c l} \cdot \prod_{j \in I}\left(A^{(j), c l}\right)^{a_{i j}}
$$

Proof. We have to calculate the Euler characteristic of the non-commutative Hilbert scheme, parameterizing representations with cyclic vector (see proof of Theorem 5.2). First, we parametrize the set of arrows between vertices $i, j \in I$ by $\alpha, 1 \leqslant \alpha \leqslant a_{i j}$. Then one can apply the action of the torus $\mathbb{G}_{m}^{\sum_{i j} a_{i j}}$ acting by rescaling arrows of the quiver. A fixed point of this action is either 
the trivial 0-dimensional representation, or it corresponds to a collection of $\sum_{j} a_{i j}$ torus-invariant representations with cyclic vectors $u_{j, \alpha}$ for $j \in I, 1 \leqslant$ $\alpha \leqslant a_{i j}$, such that the cyclic vector $v$ in the original representation is mapped to $u_{j, \alpha}$ by the arrow $i \rightarrow j$ corresponding to $\alpha$. The proof follows.

If the quiver is acyclic then all the series $A^{(i), c l}$ are rational functions, and the symplectomorphism $T$ gives a birational map. The same is true for a quiver $Q$ given by a clockwise oriented $N$-gon for any $N \geqslant 3$. It was suggested in [33], Section 8.4, that in such a situation it is natural to compose map $T$ with the antipodal involution of the classical symplectic double torus. Using the relation of symplectomorphisms and mutations as in [33] one obtains the following result, which we formulate using the standard terminology of cluster algebras (see e.g. $[19,18]$ ).

Theorem 5.4. Let $Q$ be an acyclic quiver which is obtained by mutations from a Dynkin quiver. Then the birational map given by

$$
x_{i} \mapsto \widetilde{x}_{i}:=\left(x_{i} \prod_{j} g_{j}^{a_{i j}-a_{j i}}\right)^{-1} \quad, y_{i} \mapsto \widetilde{y}_{i}:=\left(g_{i} y_{i}\right)^{-1}
$$

where $\left(g_{i}\right)_{i \in I}$ is the unique solution of the system of equations

$$
g_{i}=1+x_{i} \prod_{j} g_{j}^{a_{i j}} \in \mathbb{Z}\left[\left[\left(x_{j}\right)_{j \in I}\right]\right]
$$

induces the birational automorphisms of $\mathcal{X}$-torus with coordinates $\left(x_{i}\right)_{i \in I}$ and $\mathcal{A}$-torus with coordinates $\left(y_{i}\right)_{i \in I}$ of the order either $h+2$ or $(h+2) / 2$ depending on the Dynkin diagram, where $h$ is the Coxeter number (cf. with Coxeter automorphism from [57]).

Here $\mathcal{X}$-torus is a quotient of the double torus under the projection $\left(\left(x_{i}\right)_{i \in I},\left(y_{i}\right)_{i \in I}\right) \mapsto\left(x_{i}\right)_{i \in I}$, and $\mathcal{A}$-torus is a subtorus of the double torus given by the system of equations $x_{i}=\prod_{j \in I} y_{j}^{a_{i j}-a_{j i}}, i \in I$ (see [18] for the details).

Here we the use alleviated notation $x_{i}=\mathbf{e}_{i}^{c l}, y_{i}=\mathbf{f}_{i}^{c l}, g_{i}=A^{(i), c l}$.

The formula for the transformation from variables $\left(x_{i}\right)_{i \in I}$ to $\left(\widetilde{x}_{i}\right)_{i \in I}$ can be rewritten in a more symmetric form:

$$
x_{i}=\frac{g_{i}-1}{\prod_{j} g_{j}^{a_{i j}}}, \quad \widetilde{x}_{i}=\frac{\prod_{j} g_{j}^{a_{j i}}}{g_{i}-1} .
$$


In the next example, $Q=Q_{3}$ is the quiver with one vertex and three loops $x, y, z$, and the potential is $W=x y z-z y x$.

Proposition 5.1. For $A:=A^{\left(Q_{3}, W\right)}$ we have the following formula:

$$
A=\prod_{n, m \geqslant 1}\left(1-\mathbb{L}^{m-2} \widehat{\boldsymbol{e}}_{1}^{n}\right)^{-1} .
$$

Therefore $A^{(1), c l}=\prod_{n \geqslant 1}\left(1-\left(\boldsymbol{e}_{1}^{c l}\right)^{n}\right)^{-n}$ is the MacMahon series (cf. [3]).

Proof. It is convenient to use the duality and the approach from Section 4.8. Potential $W$ is linear in variable $z$, hence we have:

$$
D(A)=\sum_{n \geqslant 0}\left[\mathbf{H}_{\mathrm{c}}^{\bullet}\left(X_{n}\right)\right] t^{n}, t:=D\left(\widehat{\mathbf{e}}_{1}\right),
$$

where $X_{n}$ is the stack of $n$-dimensional representations of the polynomial algebra $\mathbf{k}[x, y]$. The virtual dimension of $X_{n}$ is zero.

In general, for any stack $S$ endowed with a map $w: S \rightarrow \mathbb{Z}_{\geq 0}$ (the target is a countable set understood as a stack), such that for any $k \geq 0$ the fiber $w^{-1}(k)$ is an Artin stack of finite type, we denote by $\mathbf{H}_{\mathrm{c}}^{\bullet}(S)$ an infinite series $\sum_{k \geq 0}\left[\mathbf{H}_{\mathrm{c}}^{\bullet}\left(w^{-1}(k)\right)\right] t^{k}$, with coefficients of the $\lambda$-ring $\widehat{\mathcal{M}}$. The action of $\lambda$ operations on the variable $t$ is defined as $\lambda^{\geqslant 2}(t)=0$. Hence we can write $D(A)=\left[\mathbf{H}_{\mathrm{c}}^{\bullet}(X)\right]$ where $X:=\sqcup_{n \geq 0} X_{n}, w\left(X_{n}\right):=\{n\}$.

It is a general property of $\lambda$-rings that any series $F$ of the form $1+$ $\cdots \in \widehat{\mathcal{M}}[[t]]$ can be written uniquely as $\operatorname{Sym}(G)$, where the series $G$ has zero constant term (see e.g. [31] or Section 6.1 below). We will denote $G$ by $\log _{\lambda}(F)$. Explicitly it is given by Harrison complex of a commutative algebra, see Section 6.6 for a related material.

It is convenient to introduce the following notation: for a stack $A$ endowed with a map $w: A \rightarrow \mathbb{Z}_{\geq 1}$ with fibers $w^{-1}(k), k \geq 1$ of finite type, and for another stack $B$ of fintie type, we denote

$$
(p t \sqcup A)^{B}:=\sqcup_{n \geq 0}\left(A^{n} \times\left(B^{n}-\operatorname{Diag}_{n}^{B}\right)\right)_{\operatorname{Sym}_{n}}
$$

by analogy with the Newton formula

$$
(1+a)^{b}=\sum_{n \geq 0} \frac{a^{n} \cdot b(b-1) \ldots(b-n+1)}{n !} .
$$


Here $\operatorname{Diag}_{n}^{B} \subset B^{n}$ is the union of all diagonals, and the "weight" function $w$ is defined on $(p t \sqcup A)^{B}$ by the formula

$$
\left.w\left(\left[a_{1}, \ldots, a_{n}, b_{1}, \ldots, b_{n}\right)\right]\right)=\sum_{i} w\left(a_{i}\right) .
$$

Then one can check easily that

$$
\log _{\lambda}\left(\left[\mathbf{H}_{\mathrm{c}}^{\bullet}\left((p t \sqcup A)^{B}\right)\right]\right)=\left[\mathbf{H}_{\mathrm{c}}^{\bullet}(B)\right] \cdot \log _{\lambda}\left(\left[\mathbf{H}_{\mathrm{c}}^{\bullet}(p t \sqcup A)\right]\right) .
$$

Now we apply this formula to our situation. Let us denote by $X_{+}^{(0)} \subset$ $\sqcup_{n \geqslant 0} X_{n}$ the substack consisting of nilpotent representations of $\mathbf{k}[x, y]$ (i.e., for any $n \geq 1$ both $x, y$ act in the $n$-dimensional representation by operators whose $n$th power is zero). Then we have a constructible isomorphism of $\mathbb{Z}_{\geq 0}$-graded stacks

$$
\left(p t \sqcup X_{+}^{(0)}\right)^{\mathbb{A}_{\mathbf{k}}^{2}} \simeq X .
$$

The reason for this identity is that any finite-dimensional $\mathbf{k}[x, y]$-module is canonically isomorphic to a direct sum of shifts of non-trivial nilpotent modules by $\left(x \mapsto x+c_{1}, y \mapsto y+c_{2}\right)$ with where $\left(c_{1}, c_{2}\right) \in \mathbb{G}_{a, \mathbf{k}}^{2}$ are different points of a finite subset of $\mathbb{A}_{\mathbf{k}}^{2}$. This implies the identity

$$
\log _{\lambda}\left(\sum_{n \geqslant 0}\left[\mathbf{H}_{\mathbf{c}}^{\bullet}\left(X_{n}\right)\right] t^{n}\right)=\mathbb{L}^{2} \log _{\lambda}\left(\sum_{n \geqslant 0}\left[\mathbf{H}_{\mathbf{c}}^{\bullet}\left(X_{n}^{(0)}\right)\right] t^{n}\right),
$$

where the factor $\mathbb{L}^{2}$ is equal to $\left[\mathbf{H}_{\mathbf{c}}^{\bullet}\left(\mathbb{A}_{\mathbf{k}}^{2}\right)\right]$ and $X_{n}^{(0)}=X^{(0)} \cap X_{n}$.

Similarly, we have

$$
\log _{\lambda}\left(\sum_{n \geqslant 0}\left[\mathbf{H}_{\mathrm{c}}^{\bullet}\left(X_{n}^{(1)}\right)\right] t^{n}\right)=(\mathbb{L}-1) \log _{\lambda}\left(\sum_{n \geqslant 0}\left[\mathbf{H}_{\mathrm{c}}^{\bullet}\left(X_{n}^{(0)}\right)\right] t^{n}\right),
$$

where $X^{(1)}$ denotes the stack of representations of $\mathbf{k}[x, y]$ in which $x$ is nilpotent and $y$ is invertible. We claim that

$$
\sum_{n \geqslant 0}\left[\mathbf{H}_{\mathbf{c}}^{\bullet}\left(X_{n}^{(1)}\right)\right] t^{n}=\prod_{n \geqslant 1}\left(1-t^{n}\right)^{-1} .
$$

Indeed, the contribution of any stratum of $X_{n}^{(1)}$ corresponding to a given conjugacy class of the nilpotent operator $x$ (i.e., corresponding to a partition of $n)$ is equal to $\left[\mathbf{H}_{\mathrm{c}}^{\bullet}\left(G_{x} / G_{x}\right)\right]$, where $G_{x}$ is the normalizer of $x$ in $\mathrm{GL}_{\mathbf{k}}(n)$, 
and we consider the stack $G_{x} / G_{x}$ given by the adjoint action of $G_{x}$ on itself. Group $G_{x}$ is the group of invertible elements in a finite-dimensional associative algebra, hence it is an extension of the product of general linear groups by a unipotent group. We can use Lemma 5.2 from Section 5.3 for the calculation of the class, and obtain the equality

$$
\left[\mathbf{H}_{\mathrm{c}}^{\bullet}\left(G_{x} / G_{x}\right)\right]=1
$$

Therefore, the generating series for $\left[\mathbf{H}_{\mathrm{c}}^{\bullet}\left(X_{n}^{(1)}\right)\right], n \geq 0$ is just the usual generating series for partitions. This implies the formula

$$
\log _{\lambda}\left(\sum_{n \geqslant 0}\left[\mathbf{H}_{\mathrm{c}}^{\bullet}\left(X_{n}^{(1)}\right)\right] t^{n}\right)=\sum_{n \geqslant 1} t^{n}
$$

and hence

$$
D(A)=\operatorname{Sym}\left(\mathbb{L}^{2} /(\mathbb{L}-1) \cdot \sum_{n \geqslant 1} t^{n}\right)=\prod_{n, m \geqslant 1}\left(1-\mathbb{L}^{2-m} t^{n}\right)^{-1}
$$

Applying the duality $D$ we obtain the desired formula for $A$.

In the final example quiver $Q$ has three vertices $I=\{1,2,3\}$, six arrows $\alpha_{12}, \alpha_{23}, \alpha_{31}, \beta_{12}, \beta_{23}, \beta_{31}$ and potential

$$
W=\alpha_{31} \cdot \alpha_{23} \cdot \alpha_{12}+\beta_{31} \cdot \beta_{23} \cdot \beta_{12}
$$

This example is interesting because it is invariant under mutations (cf. [33], Section 8.4). Again, using the fact that $W$ is linear in $\alpha_{31}, \beta_{31}$, we reduce the question to the calculation of $\left[\mathbf{H}_{\mathrm{c}}^{\bullet}\left(S_{\gamma}\right)\right]$, where $S_{\gamma}, \gamma \in \mathbb{Z}_{\geqslant 0}^{3}$ is the stack of representations of $Q$ of dimension vector $\gamma$ with removed arrows $\alpha_{31}, \beta_{31}$, and relations $\alpha_{23} \cdot \alpha_{12}=\beta_{23} \cdot \beta_{12}=0$. It has the same cohomology as a similar stack for the quiver $Q^{\prime}$ with five vertices $I=1,1^{\prime}, 2,3,3^{\prime}$, six arrows $\alpha_{12}, \alpha_{23}, \beta_{1^{\prime} 2}, \beta_{23}, \beta_{23^{\prime}}, \delta_{11^{\prime}}, \delta_{33^{\prime}}$ with relations $\alpha_{23} \cdot \alpha_{12}=0, \beta_{23^{\prime}}$. $\beta_{1^{\prime} 2}=0$ and conditions that $\delta_{11^{\prime}}, \delta_{33^{\prime}}$ are invertible. If one removes from $Q^{\prime}$ the arrows $\delta_{11^{\prime}}, \delta_{33^{\prime}}$ (which corresponds to the division by a simple factor $\left.\left[\mathbf{H}_{\mathrm{c}}^{\bullet}\left(G L\left(\gamma^{1}\right) \times G L\left(\gamma^{2}\right)\right)\right]\right)$ then one obtains a tame problem of linear algebra 
with 13 indecomposable objects and with dimension vectors

$$
\left\{\left(\gamma^{1}, \gamma^{1^{\prime}}, \gamma^{2}, \gamma^{3}, \gamma^{3^{\prime}}\right) \mid \gamma^{i} \in\{0,1\}, \gamma^{1} \gamma^{2} \gamma^{3}=\gamma^{1^{\prime}} \gamma^{2} \gamma^{3^{\prime}}=0\right\}
$$

The result is a complicated sum (over 13 indices) of $q$-hypergeometric type. We do not know yet an explicit formula for the symplectomorphism $T$.

We should warn the reader that this example does not coincide with the one from [33], Section 8.4. Namely, the mutation of quivers in the sense of Fomin and Zelevinsky corresponds to quivers with generic formal potentials. One of representatives of such a potential is

$$
W^{\prime}:=W+\alpha_{31} \cdot \beta_{23} \cdot \alpha_{12} \cdot \beta_{31} \cdot \alpha_{23} \cdot \beta_{12}
$$

Incidentally, such apotential is also polynomial and invariant under mutations (simultaneously as polynomial and as a formal power series). For potentials which are formal series one should use another cohomology theory (critical cohomology from Section 8). The motivic DT-series for $(Q, W)$ and $\left(Q, W^{\prime}\right)$ differ by some unknown factor $1+\cdots \in \widetilde{\mathcal{M}}\left[\left[\mathbf{e}_{111}\right]\right]$ in central variable $\mathbf{e}_{111}$.

\subsection{Mutations of quivers with potentials}

Here we study an application of the product formula to the tilting (mutation) of quivers with polynomial potentials. Results of this Section will not be used in the rest of the paper, so the reader can jump to Section 6.1.

Let $(Q, W)$ be a quiver with potential. We fix a vertex $i_{0} \in I$ of $Q$ which is loop-free (i.e., $a_{i_{0} i_{0}}=0$ for the incidence matrix $\left.\left(a_{i j}\right)_{i, j \in I}\right)$. We will write the potential $W$ as a finite k-linear combination of cycles (in other words, cyclic paths) $\sigma$ in $Q$

$$
W=\sum_{\sigma} c_{\sigma} \sigma,
$$

where $\mathbf{k}$ is the ground field. For any vertex $i \in I$ we have the corresponding cycle $(i)$ of length 0 (the image of the projector corresponding to $i$ ).

We define below the (right) "mutation" $\left(Q^{\prime}, W^{\prime}\right)$ of $(Q, W)$ at the vertex $i_{0}$. Our goal in this section is to establish a relation between generating series $A^{(Q, W)}$ and $A^{\left(Q^{\prime}, W^{\prime}\right)}$ similar to the one from [33], Section 8.4.

The definition of $\left(Q^{\prime}, W^{\prime}\right)$ is similar to the standard one from [56] (in the context of quivers whose potentials are formal cyclic series).

(1) The set of vertices of $Q^{\prime}$ is the same set $I$. 
(2) The new set of arrows and new matrix $\left(a_{i j}^{\prime}\right)_{i, j \in I}$ are defined such as follows:

$a_{i_{0} i_{0}}^{\prime}=0$

$a_{i_{0} j}^{\prime}=a_{j i_{0}}, a_{j i_{0}}^{\prime}=a_{i_{0} j}$ for any $j \neq i_{0}$ (in terms of arrows: we reverse each arrow $\alpha$ which has head or tail at $i_{0}$, i.e., replace $\alpha$ by a new arrow $\alpha^{*}$ );

$a_{j_{1} j_{2}}^{\prime}=a_{j_{1} j_{2}}+a_{j_{1} i_{0}} a_{i_{0} j_{2}}$ for $j_{1}, j_{2} \neq i_{0}$ (in terms of arrows: for every pair of arrows $i_{0} \stackrel{\beta}{\rightarrow} j_{2}, j_{1} \stackrel{\alpha}{\rightarrow} i_{0}$, we create a new arrow $\left.j_{1} \stackrel{[\beta \alpha]}{\rightarrow} j_{2}\right)^{10}$.

(3) The mutated potential $W^{\prime}$ is defined as a sum of three terms:

$$
W^{\prime}=W_{1}+W_{2}+W_{3}
$$

where

$$
\begin{aligned}
& W_{1}=\sum_{\substack{j_{1} \stackrel{\alpha}{\rightarrow} i_{0} \stackrel{\beta}{\rightarrow} j_{2} \\
W_{2}}} \beta^{*} \cdot[\beta \alpha] \cdot \alpha^{*}, \\
& \sum_{\sigma \neq\left(i_{0}\right)} c_{\sigma} \sigma^{\mathrm{mod}}, \\
& W_{3}=c_{\left(i_{0}\right)}\left(-\left(i_{0}\right)+\sum_{j \stackrel{\alpha}{\rightarrow} i_{0}}(j)\right)=c_{\left(i_{0}\right)}\left(-\left(i_{0}\right)+\sum_{j \in I} a_{j i_{0}}(j)\right) .
\end{aligned}
$$

Let us explain the notation in the formulas for $W_{i}, i=1,2,3$.

The summand $W_{1}$ consists of cubic terms generated by cycles from $i_{0}$ to $i_{0}$ of the form $\beta^{*} \cdot[\beta \alpha] \cdot \alpha^{*}$, and it can be thought of as a "Lagrange multiplier".

The summand $W_{2}$ is obtained from $W$ by modifying each cycle $\sigma$ (except $\left.\left(i_{0}\right)\right)$ to a cycle $\sigma^{\text {mod }}$ such as follows: for each occurrence of $i_{0}$ in the cycle $\sigma$ (there might be several of them) we replace the consecutive two-arrow product $\left(i_{0} \stackrel{\beta}{\rightarrow} j^{\prime}\right) \cdot\left(j \stackrel{\alpha}{\rightarrow} i_{0}\right)$ of $\sigma$ by an arrow $j \stackrel{[\beta \alpha]}{\rightarrow} j^{\prime}$. New cycle is denoted by $\sigma^{\mathrm{mod}}$, and it is taken with the same coefficient $c_{\sigma}$. In particular, if $\sigma$ does not contain $i_{0}$ then $\sigma^{\text {mod }}=\sigma$. This modification procedure is not applicable only to the cycle $\left(i_{0}\right)$ of zero length.

The last summand $W_{3}$ can be thought of as a modification of the term in $W$ corresponding to the exceptional cycle $\left(i_{0}\right)$. There is also a version of $W_{3}$ for the left mutation in which the same sum is taken over all arrows with the head (and not the tail) at $i_{0}$.

\footnotetext{
${ }^{10}$ Since we use left $\mathbf{k} Q$-modules our notation for the composition of arrows is compatible with the notation for the composition of linear operators.
} 
Our goal in this section is to establish a relation between generating series $A^{(Q, W)}$ and $A^{\left(Q^{\prime}, W^{\prime}\right)}$ similar to the one from [33], Section 8.4. Each of the two series uniquely determines the other one.

Let us choose a central charge $Z: \mathbb{Z}_{\geqslant 0}^{I} \rightarrow \overline{\mathbb{H}}_{+}$, where $\mathbb{H}_{+}$is the (open) upper-half plane. It is completely determined by its values on the standard basis vectors, so we identify $Z$ with a collection of complex numbers $\left(z_{i}\right)_{i \in I}$ belonging to $\mathbb{H}_{+}$. We impose the condition that

$$
\operatorname{Arg}\left(z_{i_{0}}\right)>\operatorname{Arg}\left(z_{i}\right), \forall i \neq i_{0}
$$

Denote by $\mathcal{H}^{(Q, W)}$ the Cohomological Hall algebra corresponding to $(Q, W)$ and by $\mathcal{H}_{V}^{(Q, W)}$ the Cohomological Hall vector space corresponding to a subsector $V \subset \mathbb{H}_{+}$, with the apex at the origin. For $V=l$ (a ray in $\mathbb{H}_{+}$) this vector space is in fact a Cohomological Hall algebra, as we explained before. Let us introduce a ray $l_{0}=\mathbb{R}_{>0} z_{i_{0}}$, and a sector $V_{0,+}=\left\{z \in \mathbb{H}_{+} \mid \operatorname{Arg}(z)<\right.$ $\left.\operatorname{Arg}\left(z_{i_{0}}\right)\right\}$. The Factorization formula implies

$$
A^{(Q, W)}=A_{l_{0}}^{(Q, W)} A_{V_{0,+}}^{(Q, W)}
$$

We observe that $\mathcal{H}_{l_{0}}^{(Q, W)}$ corresponds to the category of representations of $Q$ supported at the vertex $i_{0}$. Since it is the same as the category of representations of the quiver $A_{1}$ with trivial potential, we can use the results of Section 2 and see that $\mathcal{H}_{l_{0}}^{(Q, W)}$ is a free exterior algebra, and

$$
A_{l_{0}}^{(Q, W)}=\left(-\mathbf{e}_{i_{0}} ; \mathbb{L}\right)_{\infty}=\left(\mathbb{L}^{1 / 2} \widehat{\mathbf{e}}_{i_{0}} ; \mathbb{L}\right)_{\infty}
$$

Similarly, let us choose for the quiver $Q^{\prime}$ a central charge $Z^{\prime}$ such that for the corresponding collection of complex numbers $\left(z_{i}^{\prime}\right)_{i \in I} \in \mathbb{H}_{+}$the inequalities

$$
\operatorname{Arg}\left(z_{i}^{\prime}\right)>\operatorname{Arg}\left(z_{i_{0}}^{\prime}\right), \forall i \neq i_{0}
$$

hold. Consider the ray $l_{0}^{\prime}:=\mathbb{R}_{>0} z_{i_{0}}^{\prime}$ and sector $V_{0,-}^{\prime}:=\left\{z \in \mathbb{H}_{+} \mid \operatorname{Arg}(z)>\right.$ $\left.\operatorname{Arg}\left(z_{i_{0}}^{\prime}\right)\right\}$. Then we use again the Factorization formula

$$
A^{\left(Q^{\prime}, W^{\prime}\right)}=A_{V_{0,-}^{\prime}}^{\left(Q^{\prime}, W^{\prime}\right)} A_{l_{0}^{\prime}}^{\left(Q^{\prime}, W^{\prime}\right)}
$$

and the equality

$$
A_{l_{0}^{\prime}}^{\left(Q^{\prime}, W^{\prime}\right)}=\left(\mathbb{L}^{1 / 2} \widehat{\mathbf{e}}_{i_{0}}^{\prime} ; \mathbb{L}\right)_{\infty}
$$

Comparison of the generating functions for $(Q, W)$ and $\left(Q^{\prime}, W^{\prime}\right)$ is based on the following result. 
Proposition 5.2. Let $\gamma, \gamma^{\prime} \in \mathbb{Z}^{I}$ are related as

$$
\left(\gamma^{\prime}\right)^{i}=\gamma^{i} \text { for } i \neq i_{0}, \quad\left(\gamma^{\prime}\right)^{i_{0}}=\sum_{j} a_{i_{0} j} \gamma^{j}-\gamma^{i_{0}}
$$

Then there is an isomorphism of graded cohomology spaces

$$
\mathcal{H}_{V_{0,+}, \gamma}^{(Q, W), \bullet} \otimes \mathbb{T}^{\sum_{j} a_{j i_{0}} \gamma^{j}\left(\gamma^{\prime}\right)^{i_{0}}} \simeq \mathcal{H}_{V_{0,-}^{\prime}, \gamma^{\prime}}^{\left(Q^{\prime}, W^{\prime}\right), \bullet}
$$

Corollary 5.1. After the identification of quantum tori

$$
\widehat{\boldsymbol{e}}_{\gamma} \leftrightarrow \widehat{\boldsymbol{e}}_{\gamma^{\prime}}^{\prime}, \boldsymbol{e}_{\gamma} \leftrightarrow \mathbb{L}^{\sum_{j} a_{j i_{0}} \gamma^{j}\left(\gamma^{\prime}\right)^{i_{0}}} \boldsymbol{e}_{\gamma^{\prime}}^{\prime}
$$

where $\gamma$ and $\gamma^{\prime}$ are related as in the Proposition, the series $A_{V_{0,+}}^{(Q, W)}$ and $A_{\left.V_{0,-}^{\prime}, W^{\prime}\right)}^{\left(Q^{\prime}\right.}$ coincide with each other.

Corollary implies the comparison formula for $A^{(Q, W)}$ and $A^{\left(Q^{\prime}, W^{\prime}\right)}$ which coincides with the one from Section 8.4 in [33] (called there the property 3 ) of the generating series $\mathbf{E}_{Q}$ ).

Proof of Proposition. By definition,

$$
\mathcal{H}_{V_{0,+}, \gamma}^{(Q, W), \bullet}=\mathbf{H}_{\mathrm{G}_{\gamma}}\left(\mathrm{M}_{V_{0,+}, \gamma}^{Q}, W_{\gamma}\right)
$$

where $\mathrm{M}_{V_{0,+}, \gamma}^{Q} \in \mathrm{M}_{\gamma}^{Q}$ is a $\mathrm{G}_{\gamma}$-invariant Zariski open subset consisting of representations of $Q$ which do not have a non-trivial subrepresentation supported at the vertex $i_{0}$. In other words, a representation $E=\oplus_{i} E_{i}$ (over $\overline{\mathbf{k}}$ ) belongs to $\mathrm{M}_{V_{0,+}, \gamma}^{Q}$ if and only if the homomorphism

$$
E_{i_{0}} \rightarrow \oplus_{j \neq i_{0}} \oplus_{i_{0} \stackrel{\beta}{\rightarrow} j} E_{j}
$$

given by the direct sum of all arrows with tail at $i_{0}$, is a monomorphism.

Similarly, we have

$$
\mathcal{H}_{V_{0,-}^{\prime}, \gamma^{\prime}}^{\left(Q^{\prime}, W^{\prime}\right) \bullet}=\mathbf{H}_{\mathrm{G}_{\gamma^{\prime}}}\left(\mathrm{M}_{V_{0,-}^{\prime}, \gamma^{\prime}}^{Q^{\prime}}, W_{\gamma^{\prime}}^{\prime}\right)
$$

where $\mathrm{M}_{V_{0,-}^{\prime}, \gamma^{\prime}}^{Q^{\prime}} \subset \mathrm{M}_{\gamma^{\prime}}^{Q^{\prime}}$ is given by the condition that

$$
\oplus_{j \neq i_{0}} \oplus_{i_{0} \leftarrow j} E_{j}^{\prime} \rightarrow E_{i_{0}}^{\prime}
$$

is an epimorphism. 
Let us denote by $Q^{\prime \prime}$ the quiver obtained from $Q^{\prime}$ by removing all arrows $j \stackrel{\alpha^{*}}{\leftarrow} i_{0}$ with tail at $i_{0}$. The condition on a representation $E^{\prime}$ of $Q^{\prime}$ to belong to $\mathrm{M}_{V_{0}^{\prime}, \gamma^{\prime}}^{Q^{\prime}}$ depends only on the restriction of the action of $Q^{\prime}$ to $Q^{\prime \prime}$, and we get a fibration by affine spaces

$$
\pi: \mathrm{M}_{V_{0,-}^{\prime}, \gamma^{\prime}}^{Q^{\prime}} \rightarrow \mathrm{M}_{V_{0,-}^{\prime}, \gamma^{\prime}}^{Q^{\prime \prime}}
$$

Potential $W_{\gamma^{\prime}}^{\prime}$ is at most linear along fibers of $\pi$. Hence, the closed subscheme of $\mathrm{M}_{V_{0,-}^{\prime}, \gamma^{\prime}}^{Q^{\prime}}$ consisting of points at which $W_{\gamma^{\prime}}^{\prime}$ has zero derivative along the fiber of $\pi$, is the pullback by $\pi$ of a closed subscheme $Z_{\gamma^{\prime}}$. It is straightforward to see (considering terms of type $W_{1}$ in the formula for $W^{\prime}$ ) that $Z_{\gamma^{\prime}} \subset \mathrm{M}_{V_{0,-}^{\prime}, \gamma^{\prime}}^{Q^{\prime \prime}}$ is the closed $\mathrm{G}_{\gamma^{\prime}}$-invariant subscheme given by the condition

$$
\forall k \neq i_{0}, \forall k \stackrel{\alpha^{*}}{\leftarrow} i_{0}, \quad \sum_{j \neq i_{0}} \sum_{i_{0} \beta^{*}} \beta^{*} \cdot[\beta \alpha]=0 \in \operatorname{Hom}\left(E_{k}, E_{i_{0}}^{\prime}\right) .
$$

In other words, $Z_{\gamma^{\prime}}$ is given by the equation $\partial W_{1} / \partial \alpha^{*}=0, \forall k \stackrel{\alpha^{*}}{\leftarrow} i_{0}$.

Lemma 5.3. Scheme $Z_{\gamma^{\prime}}$ is smooth, and we have a natural equivalence of stacks

$$
\mathrm{M}_{V_{0,+}, \gamma}^{Q} / \mathrm{G}_{\gamma} \simeq Z_{\gamma^{\prime}} / \mathrm{G}_{\gamma^{\prime}}
$$

identifying restrictions of potentials $W_{\gamma}$ and $W_{\gamma^{\prime}}^{\prime}$, respectively.

Proof of Lemma. This is a version of the classical Gelfand-Ponomarev correspondence. Namely, if $E$ is a representation of $Q$ which belongs to $\mathrm{M}_{V_{0,+}, \gamma}^{Q} /$ $\mathrm{G}_{\gamma}{ }^{11}$, then we associate with it a representation $E^{\prime}$ of $Q^{\prime \prime}$ by

$$
E_{j}^{\prime}:=E_{j} \text { for } j \neq i_{0}, E_{i_{0}}^{\prime}:=\operatorname{Coker}\left(E_{i_{0}} \hookrightarrow \oplus_{j \neq i_{0}} \oplus_{i_{0} \stackrel{\beta}{\rightarrow} j} E_{j}\right),
$$

and the action of arrows in $Q^{\prime \prime}$ given in the following way. First, for any "old" arrow $j_{1} \rightarrow j_{2}$ with $j_{1}, j_{2} \neq i_{0}$ its action in $E$ and $E^{\prime}$ is the same. The action of arrow $\beta^{*}: E_{j_{0}}^{\prime}=E_{j_{0}} \rightarrow E_{i_{0}}^{\prime}$ for any $i_{0} \stackrel{\beta}{\rightarrow} j_{0}$ in the original quiver

\footnotetext{
${ }^{11}$ We will abuse the notation identifying a representation with the corresponding point of the stack.
} 
$Q$ is the composition

$$
E_{j_{0}} \hookrightarrow \oplus_{j \neq i_{0}} \oplus_{i_{0} \stackrel{\beta}{\rightarrow} j} E_{j} \rightarrow E_{i_{0}}^{\prime}
$$

of the natural inclusion of the direct summand, and of the projection to the cokernel. The action of $[\beta \alpha]$ in $E^{\prime}$ is defined as the composition of arrows corresponding to $\alpha$ and $\beta$ in $Q$. The dimension vector of $E^{\prime}$ is $\gamma^{\prime}$ as defined in the Proposition.

It is easy to see that $E^{\prime}$ belongs to $M_{V_{0,-}^{\prime \prime}, \gamma^{\prime}}^{Q^{\prime}} / \mathrm{G}_{\gamma^{\prime}}$, and for any arrow $\alpha: j \rightarrow i_{0}$ in $Q$ the equation

$$
\sum_{\beta} \beta^{*} \cdot[\beta \alpha]=0
$$

is satisfied. Hence $E^{\prime}$ belongs to $Z_{\gamma^{\prime}} / \mathrm{G}_{\gamma^{\prime}}$.

Conversely, for any representation $E^{\prime}$ of $Q^{\prime \prime}$ lying in $Z_{\gamma^{\prime}} / \mathrm{G}_{\gamma^{\prime}}$ we define the corresponding representation $E$ of $Q$ by

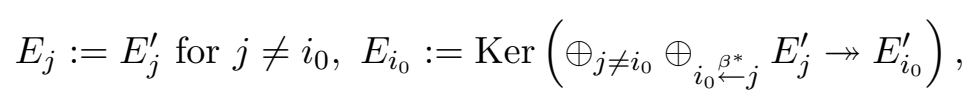

and the action of arrows of $Q$ is determined uniquely in such a way that $E^{\prime}$ is obtained from $E$ by the formulas given at the beginning of the proof.

Hence, we conclude that stacks $\mathrm{M}_{V_{0,+}, \gamma}^{Q} / \mathrm{G}_{\gamma}$ and $Z_{\gamma^{\prime}} / \mathrm{G}_{\gamma^{\prime}}$ are naturally equivalent, and therefore the scheme $Z_{\gamma^{\prime}}$ is smooth (because $\mathrm{M}_{V_{0,+}, \gamma}^{Q} / \mathrm{G}_{\gamma}$ is a smooth stack).

A straightforward check shows that on $\pi^{-1}\left(Z_{\gamma^{\prime}}\right)$ the component of $W_{\gamma^{\prime}}^{\prime}$ corresponding to the term $W_{1}$ vanishes, the component corresponding to $W_{2}$ matches $W_{\gamma}-c_{i_{0}} \operatorname{dim} E_{i_{0}}$, and the value of constant given by $W_{3}$ coincides with $c_{i_{0}} \operatorname{dim} E_{i_{0}}$.

In order to finish the proof of Proposition we apply Proposition 4.5 from Section 4.8 with the following choices of spaces and functions:

$$
X=\mathrm{M}_{V_{0,-}^{\prime}, \gamma^{\prime}}^{Q^{\prime}}, Y=\mathrm{M}_{V_{0,-}^{\prime}, \gamma^{\prime}}^{Q^{\prime \prime}}, G=\mathrm{G}_{\gamma^{\prime}}, Z=Z_{\gamma^{\prime}}, f=W_{\gamma^{\prime}}^{\prime}
$$

together with Poincaré duality for the smooth stack $Z_{\gamma^{\prime}} / \mathrm{G}_{\gamma^{\prime}}$. The shift

$$
d=\sum_{j} a_{j i_{0}} \gamma^{j}\left(\gamma^{\prime}\right)^{i_{0}}
$$

is the dimension of fibers of fibration $\pi$. Proposition is proven. 


\section{Factorization systems and integrality of exponents}

\subsection{Admissible series and integrality properties}

We will work in the framework of $\lambda$-rings (see e.g. $[25,31,40]$ ). Recall that for such a ring $B$ we have a collection of operations $\lambda^{n}: B \rightarrow B, n \geqslant 0$ satisfying the natural properties of the operations of wedge power on the $K_{0}$-group of the Tannakian category $G-\bmod$ of finite-dimensional representations of an affine algebraic group $G$ (see loc. cit. for precise definition).

Definition 6.1. An element $x$ of a $\lambda$-ring is called line element if $x$ is invertible and

$$
\lambda^{i}(x)=0, \quad \forall i \geqslant 2
$$

For example, for the $K_{0}$-ring of the tensor category of finite-dimensional representations of a pro-affine algebraic group over a field, the class of any one-dimensional representation is a line element. The set of line elements in $B$ is a subgroup of $B^{\times}$.

If $B$ is $\lambda$-ring and $t$ is a variable (which we treat as a line element) then

$$
B[t], B\left[t, t^{-1}\right], B[[t]], B((t))
$$

are again $\lambda$-rings.

Definition 6.2. For a $\lambda$-ring $B$ and a finite set $I$, a series

$$
F \in B\left(\left(q^{1 / 2}\right)\right)\left[\left[\left(x_{i}\right)_{i \in I}\right]\right]
$$

where $q^{1 / 2},\left(x_{i}\right)_{i \in I}$ are line elements (variables) is called admissible if it has a form

$$
F=\operatorname{Sym}\left(\sum_{\gamma \in \mathbb{Z}_{\geqslant 0}^{I}-\{0\}}\left(f_{\gamma} \cdot \sum_{n \geqslant 0} q^{n} \cdot \prod_{i \in I} x_{i}^{\gamma^{i}}\right)\right)
$$

where $f_{\gamma} \in B\left[q^{ \pm 1 / 2}\right]$ for any $\gamma=\left(\gamma^{i}\right)_{i \in I} \in \mathbb{Z}_{\geqslant 0}^{I}-\{0\}$, and

$$
\operatorname{Sym}(b):=\sum_{n \geqslant 0} \operatorname{Sym}^{n}(b)=\sum_{n \geqslant 0}(-1)^{n} \lambda^{n}(-b)=\left(\sum_{n \geqslant 0}(-1)^{n} \lambda^{n}(b)\right)^{-1}
$$


Coefficients of an admissible series belong to the ring

$$
B\left[q^{ \pm 1 / 2},\left(\left(1-q^{n}\right)^{-1}\right)_{n=1,2, \ldots}\right] \subset B\left(\left(q^{1 / 2}\right)\right) .
$$

In the case $B=\mathbb{Z}$ and $|I|=1$ a series $F$ in one variable $x$ with coefficients in $\mathbb{Z}\left(\left(q^{1 / 2}\right)\right)$ is admissible if and only if it can be represented as a product

$$
F=\prod_{n \geqslant 1} \prod_{i \in \mathbb{Z}}\left(q^{i / 2} x^{n} ; q\right)_{\infty}^{c(n, i)} \in 1+x \cdot \mathbb{Z}\left(\left(q^{1 / 2}\right)\right)[[x]],
$$

where $c(n, i) \in \mathbb{Z}$ for all $i, n$, and for any given $n$ we have $c(n, i)=0$ for $|i| \gg 0$. Equivalently,

$$
F=\exp \left(-\sum_{n, m \geqslant 1} \frac{f_{n}\left(q^{m / 2}\right)}{m\left(1-q^{m}\right)} x^{n m}\right)
$$

where $f_{n}=f_{n}(t)$ belongs to $\mathbb{Z}\left[t^{ \pm 1}\right]$ for all $n \geqslant 1$. The equivalence of two descriptions follows from the identity

$$
\log \left(q^{i / 2} x^{n} ; q\right)_{\infty}=-\sum_{m \geqslant 1} \frac{\left(q^{m / 2}\right)^{i}}{m\left(1-q^{m}\right)} x^{n m}
$$

It is easy to see that any series which belongs to the multiplicative group

$$
1+x \cdot \mathbb{Z}\left[q^{ \pm 1 / 2}\right][[x]]
$$

is admissible. Indeed, such a series can be written uniquely as a product

$$
\prod_{n \geqslant 1} \prod_{i \in \mathbb{Z}}\left(1-q^{i / 2} x^{n}\right)^{b(n, i)}=\prod_{n \geqslant 1} \prod_{i \in \mathbb{Z}}\left(\left(q^{i / 2} x^{n} ; q\right)_{\infty}^{b(n, i)}\left(q^{i / 2+1} x^{n} ; q\right)_{\infty}^{-b(n, i)}\right)
$$

where $b(n, i) \in \mathbb{Z} \forall i, n$, and for any given $n$ we have $b(n, i)=0$ for $|i| \gg 0$.

Admissibility of a series $F=F\left(x ; q^{1 / 2}\right) \in \mathbb{Z}\left(\left(q^{ \pm 1 / 2}\right)\right)[[x]]$ implies certain divisibility properties. Namely, let us define a new series by the formula

$$
G\left(x ; q^{1 / 2}\right):=\frac{F\left(x ; q^{1 / 2}\right)}{F\left(q x ; q^{1 / 2}\right)} \in \mathbb{Z}\left[q^{ \pm 1 / 2}\right][[x]] .
$$

Then the evaluation at $q^{1 / 2}=1$ of the series $G$ is of the form

$$
G(x ; 1):=\lim _{q^{1 / 2} \rightarrow 1} G\left(x ; q^{1 / 2}\right)=\prod_{n \geqslant 1}\left(1-x^{n}\right)^{n c(n)} \in 1+x \mathbb{Z}[[x]], c(n) \in \mathbb{Z} .
$$


Obviously, admissible series form a group under multiplication. The next result shows that the admissibility is preserved by a non-trivial transformation.

Theorem 6.1. For a given symmetric integer matrix $\mathcal{B}=\left(b_{i j}\right)_{i, j \in I}$, a series

$$
F=\sum_{\gamma \in \mathbb{Z}_{\geqslant 0}^{I}} a_{\gamma} x^{\gamma} \in B\left(\left(q^{1 / 2}\right)\right)\left[\left[\left(x_{i}\right)_{i \in I}\right]\right], x^{\gamma}:=\prod_{I} x_{i}^{\gamma^{i}}, a_{\gamma} \in B\left(\left(q^{1 / 2}\right)\right)
$$

is admissible if and only if

$$
\widetilde{F}:=\sum_{\gamma \in \mathbb{Z}_{\geqslant 0}^{I}}\left(-q^{1 / 2}\right)^{\sum_{i j} b_{i j} \gamma^{i} \gamma^{j}} a_{\gamma} x^{\gamma}
$$

is admissible.

Proof will be given in Section 6.9.

This theorem implies that one can define admissible series in quantum variables. Namely, let us assume that we are given a skew-symmetric integer bilinear form $\langle\rangle:, \mathbb{Z}^{I} \otimes \mathbb{Z}^{I} \rightarrow \wedge^{2}\left(\mathbb{Z}^{I}\right) \rightarrow \mathbb{Z}$. We define the quantum torus associated with $\langle$,$\rangle as an associative algebra over \mathbb{Z}\left(\left(q^{1 / 2}\right)\right)$ generated by the elements $\widehat{\mathbf{e}}_{\gamma}, \gamma \in \mathbb{Z}^{I}$ subject to the relations given in Section 5.3 (with the identification $\left.q^{1 / 2}=\mathbb{L}^{1 / 2}\right)$ :

$$
\widehat{\mathbf{e}}_{\gamma_{1}} \widehat{\mathbf{e}}_{\gamma_{2}}=\left(-q^{1 / 2}\right)^{-\left\langle\gamma_{1}, \gamma_{2}\right\rangle} \widehat{\mathbf{e}}_{\gamma_{1}+\gamma_{2}}, \widehat{\mathbf{e}}_{0}=1
$$

Let us choose an ordered basis $\left(\gamma_{1}, \ldots, \gamma_{k}\right), k=|I|$ of the lattice $\mathbb{Z}^{I}$. To a "quantum" series

$$
F=\sum_{\gamma \in \mathbb{Z}_{\geqslant 0}^{I}} a_{\gamma} \cdot \widehat{\mathbf{e}}_{\gamma}, a_{\gamma} \in B\left(\left(q^{1 / 2}\right)\right)
$$

we can assign the corresponding "classical" series in commuting variables $\left(x_{i}\right)_{i \in I}$ in the following way. First, we rewrite $F$ as a series in ordered monomials

$$
F=\sum_{\gamma=\sum_{i} n_{i} \gamma_{i} \in \mathbb{Z}_{\geqslant 0}^{I}} \widetilde{a}_{\gamma} \cdot \widehat{\mathbf{e}}_{\gamma_{1}}^{n_{1}} \ldots \widehat{\mathbf{e}}_{\gamma_{k}}^{n_{k}}, \widetilde{a}_{\gamma} \in B\left(\left(q^{1 / 2}\right)\right)
$$

and then replace noncommuting variables by commuting ones:

$$
F_{c l}:=\sum_{\gamma \in \mathbb{Z}_{\geqslant 0}^{I}} \widetilde{a}_{\gamma} \cdot x^{\gamma} \in B\left(\left(q^{1 / 2}\right)\right)\left[\left[\left(x_{i}\right)_{i \in I}\right]\right]
$$


For different choices of ordered bases the coefficients $\widetilde{a}_{\gamma}$ differ by the multiplication by a power of $\left(-q^{1 / 2}\right)$ with the exponent given by an integer symmetric bilinear form evaluated at $\gamma \otimes \gamma$.

Definition 6.3. We say that the quantum series $F$ is quantum admissible if for some (and hence by Theorem 6.1 any) choice of the ordered basis the corresponding classical series $F_{c l}$ is admissible.

Proposition 6.1. Let us choose a generic central charge $Z: \mathbb{Z}_{\geqslant 0}^{I} \rightarrow \mathbb{H}_{+}$. Then the set of quantum admissible series coincides with the set of products

$$
\prod_{l=\mathbb{R}>0}^{\curvearrowright} F_{l}\left(\widehat{\boldsymbol{e}}_{\left.\gamma_{0}\right)}\right),
$$

where the product in the clockwise order is taken over all rays generated by primitive vectors $\gamma_{0} \in \mathbb{Z}_{\geqslant 0}^{I}$, and $F_{l}(t)$ is an admissible series in one variable.

Proof. Denote by $\mathcal{R}_{+}$the ring of series in $\left(\widehat{\mathbf{e}}_{\gamma}\right)_{\gamma \in \mathbb{Z}_{\geqslant 0}^{I}}$ with coefficients in $B\left(\left(q^{1 / 2}\right)\right)$, and by $\mathfrak{m} \subset \mathcal{R}_{+}$the maximal ideal generated by $\widehat{\mathbf{e}}_{\gamma}, \gamma \neq 0$. Notice that the definition of quantum admissible series makes sense in all quotients $\mathcal{R}_{+} / \mathfrak{m}^{N}, N=1,2, \ldots$, and a series $F$ is admissible if and only if all its images in $\mathcal{R}_{+} / \mathfrak{m}^{N}, N \geqslant 1$ are admissible. Because of that we are going to assume that $F \in \mathcal{R}_{+} / \mathfrak{m}^{N}$ is a quantum admissible truncated series for a given $N$. Hence we can write

$$
F=\sum_{|\gamma|<N} a_{\gamma} \widehat{\mathbf{e}}_{\gamma}=1+\ldots, \text { where }|\gamma|:=\sum_{i} \gamma^{i} .
$$

If $F \neq 1$ then there exists a primitive vector $\gamma_{0} \in \mathbb{Z}_{\geqslant 0}^{I}$ with $\left|\gamma_{0}\right|<N$ such that for some $k \geqslant 1$ one has $a_{k \gamma_{0}} \neq 0$, and $\arg \left(Z\left(\gamma_{0}\right)\right)$ is the largest among all $\arg (Z(\gamma))$ for which $a_{\gamma} \neq 0, \gamma \neq 0$. Quantum admissibility of $F$ implies quantum admissibility of the following truncated series in one variable

$$
F_{\gamma_{0}}:=\sum_{\left|k \gamma_{0}\right|<N} a_{k \gamma_{0}} \widehat{\mathbf{e}}_{k \gamma_{0}} .
$$

Let us choose an ordered basis $\gamma_{1}, \ldots, \gamma_{k}, k=|I|$ of $\mathbb{Z}^{I}$ such that $\gamma_{1}=\gamma_{0}$. Notice that the multiplication from the left by any quantum admissible series $f\left(\widehat{\mathbf{e}}_{\gamma_{0}}\right)$ commutes with the correspondence $F \mapsto F_{c l}$. It follows that the series $\left(F_{\gamma_{0}}\right)^{-1} F$ is quantum admissible. Then we proceed by induction in the decreasing order of $\arg (Z(\gamma))$ for all non-zero primitive vectors $\gamma$ 
which appear in the series $F$ with non-zero coefficient $a_{\gamma}$. Since for a fixed $N$ we have only finitely many rays, this proves that $F$ is quantum admissible modulo $\mathfrak{m}^{N}$. Then, as we said above, we take the projective limit $N \rightarrow \infty$. This proves that $F$ factorizes into a clockwise ordered product over the set of rays of quantum admissible series in one variable. The converse statement (such a product is quantum admissible) can be proved in a similar way.

Proposition 6.2. The set of quantum admissible series forms a subgroup under multiplication.

Proof. As in the above proof we will consider all series modulo $\mathfrak{m}^{N}$. Let $F_{1}, F_{2}$ be quantum admissible series. Let us choose a generic central charge $Z$. Then, by the previous Proposition we have

$$
F_{1}=\prod_{l}^{\curvearrowright} F_{1, l}
$$

where the product is taken over all rays as above, and $F_{1, l}$ are quantum admissible series in one variable. It suffices to prove that for any ray $l$ and any quantum admissible series $F$ the product $F_{1, l} F$ is quantum admissible (then we proceed by induction). If $l=\mathbb{R}_{>0} Z\left(\gamma_{0}\right)$ then we choose an ordered basis $\gamma_{1}, \ldots, \gamma_{k}$ of $\mathbb{Z}^{I}$ such that $\gamma_{1}=\gamma_{0}$. As in the proof of the previous Proposition we conclude that the multiplication from the left by any quantum admissible series in $\widehat{\mathbf{e}}_{\gamma_{0}}$ preserves the set of quantum admissible series. This implies the desired statement. Similarly, one proves that if $F$ is quantum admissible then its inverse $F^{-1}$ is quantum admissible as well.

Corollary 6.1. For quantum variables $y x=q x y$ the collection of elements

$$
\prod_{(a, b) \in \mathbb{Z}_{\geqslant 0}^{2}-\{0\}} \prod_{|k| \leqslant \operatorname{const}(a, b)}\left((-1)^{a b} q^{k / 2} x^{a} y^{b} ; q\right)_{\infty}^{c(a, b ; k)}
$$

with $c(a, b ; k) \in \mathbb{Z}$, is closed under the product.

The corresponding group can be called the quantum tropical vertex group (cf. [24]) since for a quantum admissible series $F$ the automorphism $A d(F)$ in the limit $q^{1 / 2} \rightarrow 1$ gives rise to a formal symplectomorphism of the symplectic torus considered in $[34,24]$. 


\subsection{Admissibility of generating series and motivic Donaldson-Thomas invariants}

Let $R$ be an $I$-bigraded smooth algebra endowed with a potential $W$ and satisfying the Assumption of Section 3.3. Let us choose a central charge $Z: I \rightarrow \mathbb{H}_{+}$. We also fix a cohomology theory $\mathbf{H}^{\bullet}$ with values in the category $\mathcal{C}^{\mathbb{Z}-g r}$ associated with a Tannakian category $\mathcal{C}$ (see Section 3.1). Also we choose a degree +1 tensor square root $\mathbb{T}^{\otimes 1 / 2}$ of $\mathbb{T} \in O b\left(\mathcal{C}^{\mathbb{Z}-g r}\right.$ ) (see Section $2.7)$.

Let us denote by $B_{\mathcal{C}}$ the $\lambda$-ring which is the $K_{0}$-ring of the subcategory of $\mathcal{C}$ consisting of objects of weight 0 . Hence we have:

$$
K_{0}(\mathcal{C})=B_{\mathcal{C}}\left[q^{ \pm 1 / 2}\right], q^{1 / 2}:=\mathbb{L}^{1 / 2}=\left[\mathbb{T}^{\otimes 1 / 2}[1]\right]
$$

Theorem 6.2. For any sector $V \subset \mathbb{H}_{+}$the generating series $A_{V}$ is quantum admissible.

The proof will be given in Section 6.9.

Corollary 6.2. Let us assume that for some ray $l=\exp (\mathrm{i} \phi) \mathbb{R}_{>0} \subset \mathbb{H}_{+}$the restriction of the form $\chi_{R}$ to the sublattice $\Gamma_{l}:=Z^{-1}(\exp (\mathrm{i} \phi) \mathbb{R}) \subset \mathbb{Z}^{I}$ is symmetric. Then for some elements $\Omega^{\operatorname{mot}}(\gamma) \in K_{0}(\mathcal{C})$ the following formula holds

$$
\sum_{\gamma \in Z^{-1}(l)}\left[\mathcal{H}_{l, \gamma}^{\bmod }\right] x^{\gamma}=\operatorname{Sym}\left(\sum_{\gamma \in Z^{-1}(l)} \Omega^{\operatorname{mot}}(\gamma) \cdot\left[\mathbf{H}^{\bullet}\left(\mathbb{P}^{\infty}\right)\right] \cdot x^{\gamma}\right)
$$

Definition 6.4. Under the above assumptions the element $\Omega^{\text {mot }}(\gamma)$ is called motivic Donaldson-Thomas invariant of the pair $(R, W)$, stability function $Z$ and dimension vector $\gamma$.

We can apply homomorphism of $\lambda$-rings $K_{0}(\mathcal{C}) \rightarrow \mathbb{Z}\left[q^{ \pm 1 / 2}\right]$ and get the so-called refined (or quantum) Donaldson-Thomas invariants, or we can apply Euler characteristic $K_{0}(\mathcal{C}) \rightarrow \mathbb{Z}$ (i.e., evaluate at $q^{1 / 2}=1$ ) and obtain numerical Donaldson-Thomas invariants (cf. [33]).

Remark 6.1. The reader should not mix motivic DT-series and motivic DT-invariants $\Omega^{\mathrm{mot}}(\gamma)$ with motivic DT-invariants of $3 C Y$ categories introduced in [33], although the latter should be better called motivic DT-series. In fact motivic DT-series for the $3 C Y$ category which is generated by critical points of $W$ is not necessarily related to $\Omega^{\operatorname{mot}}(\gamma)$ and to the motivic 
DT-series of the algebra with potential $(R, W)$. This reflects the difference between the rapid decay cohomology used in the definition of COHA given above and the critical cohomology discussed in Section 7 and used for the definition of critical COHA. The DT-series for the latter are related to those from [33].

Recall that the assumption that $\left(\chi_{R}\right)_{\mid \Gamma_{l}}$ is symmetric (which holds e.g. for any generic central charge $Z$ ) implies that we have an ordinary associative (super) algebra $\mathcal{H}_{l}^{\text {mod }}$ graded by $\Gamma_{l} \oplus \mathbb{Z}$, where the second $\mathbb{Z}$-grading is the cohomological grading (which differs from the cohomological grading for non-modified COHA by $\left.\chi_{R}(\gamma, \gamma), \gamma \in \Gamma_{l}\right)$. The parity is given by the cohomological grading modulo 2 .

We pointed out at the end of Section 5.2 an analogy between the product decomposition by slopes from Theorem 5.1 and the Poincaré-Birkhoff-Witt theorem. In particular, the algebra $\mathcal{H}_{l}^{\text {mod }}$ can be thought of as an analog of the universal enveloping algebra for some Lie (super) algebra.

Question. Is it true that $\mathcal{H}_{l}^{\text {mod }}$ is the universal enveloping algebra $U \mathfrak{g}$, where $\mathfrak{g}=\oplus_{\gamma \in \Gamma_{l}} \mathfrak{g}_{\gamma}$ is an infinite-dimensional $\Gamma_{l} \oplus \mathbb{Z}$-graded Lie super algebra in $\mathcal{C}$, which (as $\mathbb{Z}$-graded ind-object of $\mathcal{C}$ ) has the form $\mathfrak{g}=\mathfrak{g}^{\text {prim }} \otimes$ $\left(\oplus_{n \geqslant 0} \mathbb{T}^{\otimes n}\right)$, where $\mathfrak{g}^{\text {prim }}$ has finite-dimensional graded components for each grading $\gamma \in \Gamma_{l} \cap \mathbb{Z}_{\geqslant 0}^{I}$ ?

This question is closely related with Conjecture 2.1 from Section 2.6. The main evidence in favor of the positive answer to the above Question is Theorem 6.2. In all examples which we have studied so far the algebras $\mathcal{H}_{l}^{\text {mod }}$ for generic $Z$ were in fact supercommutative. Hence the hypothetical Lie super algebra $\mathfrak{g}$ is abelian in these examples.

\subsection{Equivariant cohomology with respect to maximal tori}

Let $R$ be a smooth $I$-bigraded algebra over $\mathbf{k}$, and $\mathbf{H}^{\bullet}$ be a cohomology theory with values in the tensor category of $\mathbb{Z}$-graded vector spaces over the field $K, \operatorname{char}(K)=0$ used in the definition of the Cohomological Hall algebra. Then for every $\gamma \in \mathbb{Z}_{\geqslant 0}^{I}$ the space $\mathcal{H}_{\gamma}$ is a module over $\mathbf{H}^{\bullet}\left(\mathrm{BG}_{\gamma}\right)$.

Let us introduce a more refined version of the graded component, namely

$$
\mathcal{H}_{\gamma}^{\prime}:=\mathbf{H}_{\mathbf{\top}_{\gamma}}^{\bullet}\left(\mathrm{M}_{\gamma}\right)
$$


where

$$
\mathrm{T}_{\gamma}=\prod_{i \in I}\left(\mathbb{G}_{m, \mathbf{k}}\right)^{\gamma^{i}} \subset \mathrm{G}_{\gamma}=\prod_{i \in I} \mathrm{GL}_{\mathbf{k}}\left(\gamma_{i}\right)
$$

is the canonical maximal algebraic torus in $\mathrm{G}_{\gamma}$. The Weyl group of $\mathrm{G}_{\gamma}$, which is the product of symmetric groups

$$
\operatorname{Sym}_{\gamma}:=\prod_{i \in I} \operatorname{Sym}_{\gamma^{i}}
$$

acts on $\mathrm{T}_{\gamma}$ and on $\mathrm{M}_{\gamma}$. Also $\mathcal{H}_{\gamma}^{\prime}$ is a module over the $\operatorname{ring} \mathbf{H}^{\bullet}\left(\mathrm{B} \mathrm{T}_{\gamma}\right)$, which is the polynomial ring with $\sum_{i} \gamma^{i}$ generators in degree 2. We see that $\mathcal{H}_{\gamma}^{\prime}$, treated as a super vector space over $K$ (we reduce the cohomological $\mathbb{Z}$-grading on $\mathcal{H}_{\gamma}^{\prime}$ to $\mathbb{Z} / 2 \mathbb{Z}$-grading), is the space of sections of a $\mathrm{Sym}_{\gamma^{-}}$ equivariant super quasi-coherent sheaf $\mathcal{F}_{\gamma}$ on

$$
\mathbb{A}_{K}^{\gamma}:=\prod_{i \in I} \mathbb{A}_{K}^{\gamma^{i}}
$$

The latter affine space can be considered as the space of configurations of $\sum_{i} \gamma^{i}$ points in the affine line $\mathbb{A}_{K}^{1}$ over $K$. The original space $\mathcal{H}_{\gamma}$ coincides with the space of $\operatorname{Sym}_{\gamma}$-invariants in $\mathcal{H}_{\gamma}^{\prime}$ (because $\mathbf{H}^{\bullet}\left(\mathrm{G}_{\gamma} /\left(\operatorname{Sym}_{\gamma} \ltimes \mathrm{T}_{\gamma}\right)\right)=$ $\left.\mathbf{H}^{\bullet}(p t)\right)$, and it is a module over

$$
\mathbf{H}^{\bullet}\left(\mathrm{B} \mathrm{G}_{\gamma}\right)=\left(\mathbf{H}^{\bullet}\left(\mathrm{B} \mathrm{T}_{\gamma}\right)\right)^{\mathrm{Sym}_{\gamma}} .
$$

Lemma 6.1. Space $\mathcal{H}_{\gamma}^{\prime}$ is a finitely generated $\mathbf{H}^{\bullet}\left(\mathrm{B} \mathrm{T}_{\gamma}\right)$-module.

Equivalently, the super quasi-coherent sheaf $\mathcal{F}_{\gamma}$ is coherent. Lemma implies also that $\mathcal{H}_{\gamma}$ is a finitely generated $\mathbf{H}^{\bullet}\left(\mathrm{B} \mathrm{G}_{\gamma}\right)$-module.

Proof. Notice that the stabilizer in $\mathrm{T}_{\gamma}$ of any point in $\mathrm{M}_{\gamma}$ is a connected subtorus of $\mathrm{T}_{\gamma}$, and for a given $\gamma$ there are finitely many different stabilizers $\mathrm{T}^{\prime} \subset \mathrm{T}_{\gamma}$. Consider the finite increasing filtration $\mathrm{M}_{\gamma}^{\leqslant k}$ of $\mathrm{M}_{\gamma}$ by $\mathrm{T}_{\gamma^{-}}$ invariant Zariski open subspaces consisting of points for which the dimension of the stabilizer is at most $k, k=0, \ldots, \operatorname{dim} \mathrm{T}_{\gamma}$. Let us calculate first term of the corresponding spectral sequence. The contribution of the locus $\mathrm{M}_{\gamma}^{=\mathrm{T}^{\prime}}$ of points in $\mathrm{M}_{\gamma}$ with stabilizer exactly equal to some $\mathrm{T}^{\prime}$ is

$$
\mathbf{H}^{\bullet}\left(\mathrm{B} \mathrm{T}^{\prime}\right) \otimes \mathbf{H}^{\bullet}\left(\left(\mathrm{M}_{\gamma}^{=\mathrm{T}^{\prime}} / \mathrm{T}_{\gamma}\right)^{\text {coarse }}\right) \otimes \mathbb{T}^{\otimes \operatorname{codim} \mathrm{M}_{\gamma}^{=\mathrm{T}^{\prime}}}
$$

by Thom isomorphism. Here $\left(\mathrm{M}_{\gamma}^{=\mathrm{T}^{\prime}} / \mathrm{T}_{\gamma}\right)^{\text {coarse }}$ is the scheme-theoretical quotient, not a stack. This is obviously a finitely generated module because 
$\operatorname{dim} \mathbf{H}^{\bullet}\left(\left(\mathrm{M}_{\gamma}^{=\mathrm{T}^{\prime}} / \mathrm{T}_{\gamma}\right)^{\text {coarse }}\right)<\infty$. In terms of sheaves it corresponds to a trivial super vector bundle of finite rank over an affine subspace

$$
\operatorname{Spec} \mathbf{H}^{\bullet}\left(\mathrm{BT}^{\prime}\right) \subset \operatorname{Spec} \mathbf{H}^{\bullet}\left(B \mathrm{~T}_{\gamma}\right)
$$

Lemma is proven.

Obviously, all the above considerations extend to the case when we have a potential $W \in R /[R, R]$, and also when we are given a stability function $Z$ and a sector $V \subset \mathbb{H}_{+}$. We denote by $\mathcal{F}_{V, \gamma}$ the corresponding super coherent sheaves. In the next section, we axiomatize some algebraic properties of the collection of sheaves $\left(\mathcal{F}_{V, \gamma}\right)_{\gamma \in \mathbb{Z}_{\geqslant 0}^{I}-0}$ (the case $\gamma=0$ is trivial).

\subsection{Factorization systems}

Let $G$ be a pro-algebraic group over a field $K, \operatorname{char}(K)=0$, endowed with a homomorphism

$$
t^{1 / 2}: G \rightarrow \mathbb{G}_{m, K}
$$

For us the basic example will be the motivic Galois group (or its double cover) with $t^{1 / 2}$ corresponding to the square root of Tate motive $K(-1 / 2)$. We define

$$
\widetilde{G}:=G \ltimes \mathbb{G}_{a},
$$

where $G$ acts on the additive group $\mathbb{G}_{a}=\mathbb{G}_{a, K}$ via the homomorphism

$$
G \stackrel{t^{-1}}{\rightarrow} \mathbb{G}_{m}=\operatorname{Aut} \mathbb{G}_{a}
$$

We denote by $\mathbb{T}_{0}^{1 / 2}$ the representation of $G$ corresponding to the character $t^{1 / 2}$. We set $\mathbb{T}_{0}=\left(\mathbb{T}_{0}^{1 / 2}\right)^{\otimes 2}$. Also, we denote by $q^{1 / 2}$ the class of $\mathbb{T}_{0}^{1 / 2}$ in $K_{0}(G-\bmod )$.

Group scheme $\widetilde{G}$ maps to $\operatorname{Aff}(1):=\mathbb{G}_{m} \ltimes \mathbb{G}_{a}$, and therefore acts on $\mathbb{A}_{K}^{1}$ by affine transformations. Hence $\widetilde{G}$ acts naturally on any coordinate affine space $\mathbb{A}_{K}^{N}=\left(\mathbb{A}_{K}^{1}\right)^{N}, N \geqslant 0$.

In the case of the motivic Galois group the algebra of functions $\mathcal{O}\left(\mathbb{A}_{K}^{1}\right)$ is isomorphic as $G$-module to $\mathbf{H}^{\bullet}\left(\mathbb{P}^{\infty}\right)=\oplus_{n \geqslant 0} K(-n)$.

Definition 6.5. For a given finite set $I$ and pair $\left(G, t^{1 / 2}\right)$ as above, an $I$-colored factorization system is given by the following data: 
- (equivariant sheaf) for a given $\gamma=\left(\gamma^{i}\right)_{i \in I} \in \mathbb{Z}_{\geqslant 0}^{I}-0$ a $\widetilde{G} \times \operatorname{Sym}_{\gamma}$-equivariant super coherent sheaf $\mathcal{F}_{\gamma}$ on

$$
\mathbb{A}_{K}^{\gamma}:=\prod_{i \in I} \mathbb{A}_{K}^{\gamma^{i}},
$$

where the group $\operatorname{Sym}_{\gamma}=\prod_{i \in I} \mathrm{Sym}_{\gamma^{i}}$ acts by the permutation of coordinates in $\mathbb{A}_{K}^{\gamma^{i}}, i \in I$.

- (multiplication) For $\forall \gamma_{1}, \gamma_{2} \in \mathbb{Z}_{\geqslant 0}^{I}-0$ a multiplication isomorphism

$$
\mu_{\gamma_{1}, \gamma_{2}}:\left(\mathcal{F}_{\gamma_{1}} \otimes \mathcal{F}_{\gamma_{2}}\right)_{\mid U_{\gamma_{1}, \gamma_{2}}} \stackrel{\sim}{\rightarrow} \mathcal{F}_{\gamma_{1}+\gamma_{2} \mid U_{\gamma_{1}, \gamma_{2}}},
$$

where Zariski open affine subscheme

$$
U_{\gamma_{1}, \gamma_{2}} \subset \mathbb{A}_{K}^{\gamma_{1}+\gamma_{2}} \simeq \mathbb{A}_{K}^{\gamma_{1}} \times \mathbb{A}_{K}^{\gamma_{2}}
$$

is defined as

$$
\left\{\left(\left(x_{i, \alpha}\right)_{i \in I, 1 \leqslant \alpha \leqslant \gamma_{1}^{i}},\left(y_{j, \beta}\right)_{j \in I, 1 \leqslant \beta \leqslant \gamma_{2}^{j}}\right) \mid x_{i, \alpha} \neq y_{j, \beta} \forall i, \alpha, j, \beta\right\} .
$$

These data are subject to the following conditions:

- (equivariance of multiplication) $\forall \gamma_{1}, \gamma_{2} \in \mathbb{Z}_{\geqslant 0}^{I}-0$ the isomorphism $\mu_{\gamma_{1}, \gamma_{2}}$ is equivariant with respect to the action of $\widetilde{G} \times \operatorname{Sym}_{\gamma_{1}} \times \operatorname{Sym}_{\gamma_{2}}$;

- (commutativity) $\forall \gamma_{1}, \gamma_{2} \in \mathbb{Z}_{\geqslant 0}^{I}-0$ we have

$$
\mu_{\gamma_{2}, \gamma_{1}}=\sigma_{\gamma_{1}, \gamma_{2}} \circ \mu_{\gamma_{1}, \gamma_{2}} \circ \sigma_{12}
$$

where $\sigma_{\gamma_{1}, \gamma_{2}} \in \operatorname{Sym}_{\gamma_{1}+\gamma_{2}}$ is the collection of permutations for $i \in I$ acting on $\mathbb{A}_{K}^{\gamma_{1}^{i}+\gamma_{2}^{i}}$ by

$$
\left(x_{i, 1}, \ldots, x_{i, \gamma_{1}^{i}}, y_{i, 1}, \ldots, y_{i, \gamma_{2}^{i}}\right) \mapsto\left(y_{i, 1}, \ldots, y_{i, \gamma_{2}^{i}}, x_{i, 1}, \ldots, x_{i, \gamma_{1}^{i}}\right)
$$

and $\sigma_{12}$ is the standard permutation of indices;

- (associativity) $\forall \gamma_{1}, \gamma_{2}, \gamma_{3} \in \mathbb{Z}_{\geqslant 0}^{I}-0$ two isomorphisms

$$
\left(\mathcal{F}_{\gamma_{1}} \otimes \mathcal{F}_{\gamma_{2}} \otimes \mathcal{F}_{\gamma_{3}}\right)_{\mid U_{\gamma_{1}, \gamma_{2}, \gamma_{3}}} \stackrel{\sim}{\rightarrow} \mathcal{F}_{\gamma_{1}+\gamma_{2}+\gamma_{3} \mid U_{\gamma_{1}, \gamma_{2}, \gamma_{3}}}
$$

given by two ways to put brackets coincide with each other, i.e., we have in a simplified notation

$$
\mu_{\gamma_{1}+\gamma_{2}, \gamma_{3}} \circ\left(\mu_{\gamma_{1}, \gamma_{2}} \otimes \operatorname{id}_{\gamma_{3}}\right)=\mu_{\gamma_{1}, \gamma_{2}+\gamma_{3}} \circ\left(\operatorname{id}_{\gamma_{1}} \otimes \mu_{\gamma_{2}, \gamma_{2}}\right) \text {. }
$$


Here $U_{\gamma_{1}, \gamma_{2}, \gamma_{3}} \subset \mathbb{A}_{K}^{\gamma_{1}+\gamma_{2}+\gamma_{3}}$ is the intersection of pullbacks of open sets $U_{\gamma_{i}, \gamma_{j}}, 1 \leqslant i<j \leqslant 3$.

We will also need a closely related notion.

Definition 6.6. For given $I$ and $\left(G, t^{1 / 2}\right)$, a non-singular $I$-colored factorization system is a factorization system together with a $\widetilde{G} \times \operatorname{Sym}_{\gamma_{1}} \times$ $\operatorname{Sym}_{\gamma_{2}}$-equivariant extension of $\mu_{\gamma_{1}, \gamma_{2}}$ to a morphism of super coherent sheaves

$$
\mathcal{F}_{\gamma_{1}} \otimes \mathcal{F}_{\gamma_{2}} \rightarrow \mathcal{F}_{\gamma_{1}+\gamma_{2}}
$$

satisfying (in the notation of the previous definition) the constraints of commutativity and associativity on whole spaces $\mathbb{A}_{K}^{\gamma_{1}+\gamma_{2}}$ (resp. $\left.\mathbb{A}_{K}^{\gamma_{1}+\gamma_{2}+\gamma_{3}}\right)$.

\subsection{Equivariant cohomology as a factorization system}

Let us return to the collection of torus equivariant cohomology for $\gamma \neq 0$ :

$$
\mathcal{H}_{\gamma}^{\prime}=\mathbf{H}_{\mathbf{T}_{\gamma}}^{\bullet}\left(\mathrm{M}_{\gamma}\right)
$$

interpreted as global sections of super coherent sheaves $\mathcal{F}_{\gamma}$. Here we omit the potential $W$ and sector $V$ in order to simplify the notation.

Our goal in this subsection is to define a structure of non-singular factorization system on the collection of sheaves $\mathcal{F}_{\gamma}^{\prime}=\mathcal{F}_{\gamma}^{\prime} \otimes \mathbb{T}^{\otimes \sum_{i, j} a_{i j} \gamma^{i} \gamma^{j}}$, with multiplication induced in a sense by the operation of direct sum of representations.

Lemma 6.2. For any $\gamma_{1}, \gamma_{2} \in \mathbb{Z}_{\geqslant 0}^{I}-0$ there is a natural isomorphism

$$
\begin{aligned}
& \mathbf{H}_{\mathbf{T}_{\gamma_{1}+\gamma_{2}}}^{\bullet}\left(M_{\gamma_{1}+\gamma_{2}}, M_{\gamma_{1}+\gamma_{2}}-M_{\gamma_{1}} \times M_{\gamma_{2}}\right) \\
& \quad \simeq \mathbf{H}_{\boldsymbol{T}_{\gamma_{1}}}^{\bullet}\left(M_{\gamma_{1}}\right) \otimes \mathbf{H}_{\mathbf{T}_{\gamma_{2}}}^{\bullet}\left(M_{\gamma_{2}}\right) \otimes \mathbb{T}^{\otimes \sum_{i, j} a_{i j}\left(\gamma_{1}^{i} \gamma_{2}^{j}+\gamma_{1}^{j} \gamma_{2}^{i}\right)} .
\end{aligned}
$$

Proof. This is just the Thom isomorphism. Notice that $\mathrm{T}_{\gamma_{1}+\gamma_{2}}=\mathrm{T}_{\gamma_{1}} \times \mathrm{T}_{\gamma_{2}}$ and

$$
\sum_{i, j} a_{i j}\left(\gamma_{1}^{i} \gamma_{2}^{j}+\gamma_{1}^{j} \gamma_{2}^{i}\right)=\operatorname{dim} \mathrm{M}_{\gamma_{1}+\gamma_{2}}-\operatorname{dim}\left(\mathrm{M}_{\gamma_{1}} \times \mathrm{M}_{\gamma_{2}}\right)
$$


The standard map from the long exact sequence

$$
\tilde{\mu}_{\gamma_{1}, \gamma_{2}}: \mathbf{H}_{\mathrm{T}_{\gamma_{1}+\gamma_{2}}}^{\bullet}\left(\mathrm{M}_{\gamma_{1}+\gamma_{2}}, \mathrm{M}_{\gamma_{1}+\gamma_{2}}-\mathrm{M}_{\gamma_{1}} \times \mathrm{M}_{\gamma_{2}}\right) \rightarrow \mathbf{H}_{\mathrm{T}_{\gamma_{1}+\gamma_{2}}}^{\bullet}\left(\mathrm{M}_{\gamma_{1}+\gamma_{2}}\right)
$$

gives a morphism of sheaves

$$
\mathcal{F}_{\gamma_{1}} \otimes \mathcal{F}_{\gamma_{2}} \otimes \mathbb{T}^{\otimes \sum_{i, j} a_{i j}\left(\gamma_{1}^{i} \gamma_{2}^{j}+\gamma_{1}^{j} \gamma_{2}^{i}\right)} \rightarrow \mathcal{F}_{\gamma_{1}+\gamma_{2}},
$$

where $\mathbb{T}$ can be interpreted at the trivial line bundle on $\mathbb{A}_{K}^{\gamma_{1}+\gamma_{2}}$ with the equivariant $\widetilde{G}$ structure given by the character $t$.

Proposition 6.3. For any $\gamma_{1}, \gamma_{2} \in \mathbb{Z}_{\geqslant 0}^{I}-0$ the support of $\mathbf{H}^{\bullet}\left(\mathrm{B} \mathrm{T}_{\gamma_{1}+\gamma_{2}}\right)$ module

$$
\mathbf{H}_{\mathrm{T}_{\gamma_{1}+\gamma_{2}}}^{\bullet}\left(\mathrm{M}_{\gamma_{1}+\gamma_{2}}-\mathrm{M}_{\gamma_{1}} \times \mathrm{M}_{\gamma_{2}}\right)
$$

belongs to the closed subset $\mathbb{A}_{K}^{\gamma_{1}+\gamma_{2}}-U_{\gamma_{1}, \gamma_{2}}$.

Proof. We will give a proof in the case when $\operatorname{char}(\mathbf{k})=0$. The general case is similar. Using the comparison isomorphism, we may assume that $\mathbf{k}=\mathbb{C}$ and $\mathbf{H}^{\bullet}=\mathbf{H}_{\text {Betti }}^{\bullet}$. We will need the following lemma.

Lemma 6.3. Let $\mathrm{T}^{\mathrm{c}}$ be a compact torus acting on a complex algebraic variety $X$. Then for any locally closed real semi-algebraic subset $Z \subset X$ the $\mathbb{Z}$-graded dual space to the cohomology

$$
H_{c, \mathrm{~T}}^{\bullet}(Z, \mathbb{Q})
$$

is a finitely generated $H^{\bullet}\left(\mathrm{BT}^{\mathrm{c}}, \mathbb{Q}\right)$-module with the support belonging to the union of affine spaces $\operatorname{Spec} H^{\bullet}\left(\mathrm{BT}_{\alpha}^{\mathrm{c}}, \mathbb{Q}\right) \subset \operatorname{Spec} H^{\bullet}\left(\mathrm{BT}^{\mathrm{c}}, \mathbb{Q}\right)$, where $\mathrm{T}_{\alpha}^{\mathrm{c}} \subset \mathrm{T}^{\mathrm{c}}$ are stabilizers of points in $Z$.

Proof of Lemma. We can stratify $Z$ by locally closed subspaces according to the dimension of the stabilizer in $\mathrm{T}^{\mathrm{c}}$. By spectral sequences, the question reduces to the case when the stabilizer of any point $z \in Z$ is a given subgroup $\mathrm{T}_{\alpha}^{\mathrm{c}} \subset \mathrm{T}^{\mathrm{c}}$. Let us choose a "complementary" connected subtorus $\widetilde{\mathrm{T}}_{\alpha}^{\mathrm{c}} \subset \mathrm{T}$ such that the multiplication $\mathrm{T}_{\alpha}^{\mathrm{c}} \times \widetilde{\mathrm{T}}_{\alpha}^{\mathrm{c}} \rightarrow \mathrm{T}^{\mathrm{c}}$ is surjective with finite kernel. Torus $\widetilde{\mathrm{T}}_{\alpha}^{\mathrm{c}}$ acts almost freely (with finite stabilizers) on $Z$. Hence

$$
H_{c, \mathrm{~T}^{\mathrm{c}}}^{\bullet}(Z, \mathbb{Q}) \simeq H_{c, \mathrm{~T}_{\alpha}^{\mathrm{c}}}^{\bullet}\left(Z / \widetilde{\mathrm{T}}_{\alpha}^{\mathrm{c}}, \mathbb{Q}\right) \simeq H_{c, \mathrm{~T}_{\alpha}^{\mathrm{c}}}^{\bullet}(p t, \mathbb{Q}) \otimes H_{\mathrm{c}}^{\bullet}\left(Z / \widetilde{\mathrm{T}}_{\alpha}^{\mathrm{c}}, \mathbb{Q}\right) .
$$

Rational cohomology with compact support of any real semi-algebraic set is a finite-dimensional space, hence we obtain by duality a finitely generated module supported on $\operatorname{Spec} H^{\bullet}\left(\mathrm{BT}_{\alpha}^{\mathrm{c}}, \mathbb{Q}\right)$. 
In order to prove the Proposition we will apply the previous Lemma to $X=\mathrm{M}_{\gamma_{1}+\gamma_{2}}, \mathrm{~T}=\mathrm{T}_{\gamma_{1}+\gamma_{2}}$ and $Z=\mathrm{M}_{\gamma_{1}+\gamma_{2}}-\mathrm{M}_{\gamma_{1}} \times \mathrm{M}_{\gamma_{2}}$. In this case, $Z$ is an open subset of $X$, hence it is smooth. By Poincaré duality $H_{c, \mathrm{~T}}^{\bullet}(Z, \mathbb{Q})$ is dual to $H_{\top}^{\bullet}(Z, \mathbb{Q})$ (up to shift).

For any $\gamma \in \mathbb{Z}_{\geqslant 0}^{I}$ and any point in $\mathrm{M}_{\gamma}$ its stabilizer in $\mathrm{T}_{\gamma}=\mathbb{G}_{m}^{|\gamma|},|\gamma|:=$ $\sum_{i} \gamma^{i}$, corresponds to an equivalence relation $\sim$ on the finite set

$$
\{1, \ldots,|\gamma|\}=\sqcup_{i \in I}\left\{1, \ldots, \gamma^{i}\right\}
$$

and consists of sequences of scalars $\left(\lambda_{k} \in K^{\times}\right)_{1 \leqslant k \leqslant|\gamma|}$ such that $\lambda_{k}=\lambda_{k^{\prime}}$ if $k \sim k^{\prime}$. The equivalence relation is defined such as follows. The super coherent sheaf corresponding to the contribution of points with such a stabilizer is a super vector bundle on the affine subspace consisting of configurations of points $\left(x_{i, \alpha}\right)_{i \in I, 1 \leqslant \alpha \leqslant \gamma^{i}}$ such that

$$
x_{i, \alpha}=x_{i^{\prime}, \alpha^{\prime}} \text { for }(i, \alpha) \sim\left(i^{\prime}, \alpha^{\prime}\right) .
$$

For $\gamma=\gamma_{1}+\gamma_{2}$ and any point in $\mathrm{M}_{\gamma_{1}+\gamma_{2}}-\mathrm{M}_{\gamma_{1}} \times \mathrm{M}_{\gamma_{2}}$ its stabilizer corresponds to an equivalence relation which in terms of points $\left(x_{i, \alpha}\right)_{i \in I, 1 \leqslant \alpha \leqslant \gamma_{1}^{i}}$, $\left(y_{j, \beta}\right)_{j \in I, 1 \leqslant \beta \leqslant \gamma_{2}^{j}}$ means that there exist $i_{1}, \alpha_{1}, j_{1}, \beta_{1}$ such that

$$
x_{i_{1}, \alpha_{1}}=y_{j_{1}, \beta_{1}} .
$$

This is exactly the condition which defines the complement to $U_{\gamma_{1}, \gamma_{2}}$.

From the long exact sequence and the previous lemma it follows that the restriction of $\widetilde{\mu}_{\gamma_{1}, \gamma_{2}}$ to $U_{\gamma_{1}, \gamma_{2}}$ is an isomorphism.

Theorem 6.3. The collection of super coherent sheaves

$$
\mathcal{F}_{\gamma}^{\prime}:=\mathcal{F}_{\gamma} \otimes \mathbb{T}_{0}^{\otimes \sum_{i, j} a_{i j} \gamma^{i} \gamma^{j}}
$$

with the multiplication isomorphisms obtained from $\widetilde{\mu}_{\gamma_{1}, \gamma_{2}}$ by tensoring with the identity automorphism of a tensor power of $\mathbb{T}_{0}$, is a non-singular factorization system.

Proof. Straightforward. 


\subsection{From factorization systems to admissible series}

Let $\mathcal{F}=\left(\mathcal{F}_{\gamma}\right)$ be a non-singular factorization system. Then the multiplication morphisms endow the space

$$
A_{+}^{\mathcal{F}}:=\oplus_{\gamma \in \mathbb{Z}_{\geqslant 0}^{I}-0} A_{\gamma}^{\mathcal{F}}, \quad A_{\gamma}^{\mathcal{F}}:=\Gamma\left(\mathcal{F}_{\gamma}\right)^{\operatorname{Sym}_{\gamma}}
$$

with a super-commutative associative non-unital product.

Theorem 6.4. Under the above assumptions, there exists a collection of finite-dimensional super representations $\left(E_{\gamma}\right)_{\gamma \in \mathbb{Z}_{\geqslant 0}^{I}-0}$ of $G$ such that two $\mathbb{Z}_{\geqslant 0}^{I}$-graded super representations of $G$, the spaces $A_{+}^{\mathcal{F}}$ and

$$
\operatorname{Sym}_{+}\left(\oplus_{\gamma} E_{\gamma} \otimes\left(\oplus_{n \geqslant 0} \mathbb{T}^{\otimes n}\right)\right)
$$

have the same classes in $K_{0}(G-\bmod )$ in each graded component with respect to the $\mathbb{Z}_{\geqslant 0}^{I}$-grading.

Corollary 6.3. Let $G=G_{0} \times \mathbb{G}_{m, K}, B=K_{0}\left(G_{0}-\bmod \right)$. Then the series

$$
\sum_{\gamma \in \mathbb{Z}_{\geqslant 0}^{I}}\left[A_{\gamma}^{\mathcal{F}}\right] x^{\gamma}
$$

is admissible.

The proof of the Theorem is quite involved, but in the special case when $\mathcal{F}$ comes from Cohomological Hall algebra associated with some pair $(R, W)$ one can make a much shorter and clear argument which we explain in the next section. Nevertheless, we give here the proof in complete generality having in mind applications to certain generalizations of the Cohomological Hall algebra (see Section 7.6).

Before giving the proof we collect some preparatory material concerning Harrison homology. Recall (see e.g. [1] or in modern language [22]) that the homological Harrison complex of any non-unital supercommutative algebra $A$ is defined as the free Lie supercoalgebra generated by $A[1]$, with the differential arising from the product on $A$. Explicitly, we have

$$
C_{\mathrm{Harr}}(A):=\oplus_{k \geqslant 1}\left(L(k) \otimes(A[1])^{\otimes k}\right)^{\mathrm{Sym}_{k}},
$$

where $L(k)$ is a representation of $\operatorname{Sym}_{k}$ of dimension $(k-1)$ !, the tensor product of the contragredient to the representation Lie $(k)$ of the operad of 
Lie algebras, and of the sign representation, i.e.,

$$
L(k)=\operatorname{Lie}(k)^{\vee} \otimes \operatorname{sign}_{k}
$$

If $A=\operatorname{Sym}_{+}(V)$ is a free non-unital supercommutative algebra, then Harrison homology of $A$ is isomorphic to $V[1]$. In general, the non-unital symmetric algebra generated by the Harrison homology $H_{\bullet}^{\mathrm{Harr}}(A)$ (shifted by $[-1]$ ) is quasi-isomorphic to $A$, with certain explicit homotopy. Also we will use the following statement.

Lemma 6.4. Let $B, C$ be two non-unital supercommutative algebras, and

$$
A=B \oplus C \oplus(B \otimes C)
$$

with the obvious product. Then inclusions $B \hookrightarrow A$ and $C \hookrightarrow A$ induce an isomorphism

$$
H_{\bullet}^{\mathrm{Harr}}(B) \oplus H_{\bullet}^{\mathrm{Harr}}(C) \simeq H_{\bullet}^{\mathrm{Harr}}(A)
$$

Idea of the proof. Using spectral sequence one can see that the statement of Lemma follows from the special case when multiplication on $B$ and $C$ vanishes. Hence we have a purely operadic identity.

Proof of Theorem 6.4. In our case there is a $\mathbb{Z}_{\geqslant 0}^{I}-\{0\}$-grading of both: of the algebra $A=A_{+}^{\mathcal{F}}$ and of its Harrison complex. This grading ensures an appropriate finiteness, and hence we have the following identity in the completed $K_{0}$-group:

$$
\sum_{\gamma}\left[A_{+, \gamma}^{\mathcal{F}}\right] x^{\gamma}=\operatorname{Sym}_{+}\left(-\sum_{\gamma}\left[H_{\bullet, \gamma}^{\operatorname{Harr}}\left(A_{+}^{\mathcal{F}}\right)\right] x^{\gamma}\right) .
$$

Component of degree $\gamma$ of the Harrison complex of $A^{\mathcal{F}}$ is the space of global sections of a complex of $\widetilde{G}$-equivariant super coherent sheaves on the quotient scheme

$$
X_{\gamma}:=\mathbb{A}_{K}^{\gamma} / \operatorname{Sym}_{\gamma}
$$

Proposition 6.4. For any $\gamma \neq 0$ super vector space $H_{\bullet, \gamma}^{\operatorname{Harr}}\left(A_{+}^{\mathcal{F}}\right)$ is a super coherent sheaf on $X_{\gamma}$ supported on the main diagonal

$$
X_{\gamma}^{\operatorname{diag}} \simeq \mathbb{A}_{K}^{1} \subset X_{\gamma}
$$


Proof of Proposition. Recall that our factorization systems are defined over the field $K$ of characteristic 0 . Hence, we can safely assume that $K=\mathbb{C}$. In what follows, it will be convenient for us to use analytic topology ${ }^{12}$ on $\mathbb{A}^{1}(\mathbb{C})=\mathbb{C}$.

Let $U \subset \mathbb{C}$ be an open subset. We define a nuclear $\mathbb{Z}_{\geqslant 0}^{I}$-graded non-unital Fréchet super algebra $A_{U}^{\mathcal{F}}$ by taking spaces of analytic sections over powers of $U$. We define analytic Harrison complex of $A_{U}^{\mathcal{F}}$ by taking completed tensor products. Obviously $H_{\bullet, \gamma, a n}^{\mathrm{Harr}}\left(A_{U}^{\mathcal{F}}\right)$ coincides with the space of analytic sections of the algebraic super coherent sheaf corresponding to $H_{\bullet, \gamma}^{\mathrm{Harr}}\left(A^{\mathcal{F}}\right)$, over the Stein subset $U_{\gamma} \subset X_{\gamma}^{a n}(\mathbb{C})$ consisting of configurations of points on $U$.

Let $x \in X_{\gamma}$ be a point which does not belong to the main diagonal. Then $x$ corresponds to a finite subset $S \subset \mathbb{C}$ which has at least two elements. Therefore, we can find two disjoint non-empty sets $U^{\prime}, U^{\prime \prime} \subset \mathbb{C}$ such that

$$
x \notin U_{\gamma}^{\prime}, x \notin U_{\gamma}^{\prime \prime}, x \in U_{\gamma} \text { for } U:=U^{\prime} \cup U^{\prime \prime} .
$$

Then we have

$$
A_{U}^{\mathcal{F}} \simeq A_{U^{\prime}}^{\mathcal{F}} \oplus A_{U^{\prime \prime}}^{\mathcal{F}} \oplus\left(A_{U^{\prime}}^{\mathcal{F}} \widehat{\otimes} A_{U^{\prime \prime}}^{\mathcal{F}}\right)
$$

Applying Lemma 6.4 we conclude that the Harrison cohomology of $A_{U}^{\mathcal{F}}$ in degree $\gamma \in \mathbb{Z}^{I}$ corresponds to a super coherent sheaf supported on $U_{\gamma}^{\prime} \cup U_{\gamma}^{\prime \prime}$. In particular, it vanishes in a neighborhood of $x$. Therefore $H_{\bullet, \gamma}^{\mathrm{Harr}}\left(A^{\mathcal{F}}\right)$ vanishes near $x$. We proved that $H_{\bullet, \gamma}^{\operatorname{Harr}}\left(A^{\mathcal{F}}\right)$ is supported on the main diagonal.

Any $\widetilde{G}$-equivariant super coherent sheaf on $X_{\gamma}$ supported on the main diagonal is invariant under the action of the shift group $\mathbb{G}_{a} \simeq X_{\gamma}^{\text {diag }}$. Hence it admits a canonical finite filtration with quotients isomorphic to sums of copies of $\mathcal{O}\left(X_{\gamma}^{\mathrm{diag}}\right)$. Moreover, as $G^{(2)}$-modules these quotients are of the form

$$
E \otimes \mathcal{O}\left(X_{\gamma}^{\text {diag }}\right)=E \otimes\left(\oplus_{n \geqslant 0} \mathbb{T}^{\otimes n}\right)
$$

where $E$ is a finite-dimensional representation of $G$. Hence $H_{\bullet, \gamma}^{\operatorname{Harr}}\left(A_{+}^{\mathcal{F}}\right)$ has the same class in the completed $K_{0}$-ring of $G^{(2)}$ as $E_{\gamma} \otimes\left(\oplus_{n \geqslant 0} \mathbb{T}_{0}\right)^{\otimes n}$ for some finite-dimensional $G$-module $E_{\gamma}$. This concludes the proof of Theorem 6.4.

\footnotetext{
${ }^{12} \mathrm{~A}$ purely algebraic proof exists, but it is less transparent.
} 


\subsection{Admissibility in the geometric case}

Let us assume that we are given a smooth $I$-bigraded algebra $R / \mathbf{k}$ with potential $W$ and a cohomology theory $\mathbf{H}^{\bullet}$. All the arguments below will work also if we fix a central charge $Z$ and a sector $V$. In order to shorten the formulas, we will skip $V$ from the notation.

Definition 6.7. A non-trivial representation of $R$ in coordinate spaces is called $T$-indecomposable if it can not be decomposed into the direct sum of two non-trivial subrepresentations in coordinate subspaces.

The set of $T$-indecomposable representations of $R$ with dimension vector $\gamma$ is a Zariski open subset (hence smooth)

$$
\mathrm{M}_{\gamma}^{T-\text { ind }} \subset \mathrm{M}_{\gamma}
$$

which is invariant under the action of $\operatorname{Sym}_{\gamma} \ltimes T_{\gamma}$.

Let us consider the ind-Artin stack

$$
\sqcup_{\gamma \in \mathbb{Z}_{\geqslant 0}^{I}} \mathrm{M}_{\gamma} /\left(\operatorname{Sym}_{\gamma} \ltimes \mathrm{T}_{\gamma}\right)
$$

It carries a natural stratification with smooth strata by the dimension of the stabilizer. Strata are labeled by sequences $\left(\gamma_{1}, \ldots, \gamma_{k}\right), k \geqslant 1$ of non-zero vectors $\gamma_{i} \in \mathbb{Z}_{\geqslant 0}^{I}$ such that $\sum_{i=1}^{k} \gamma_{i}=\gamma$, up to the action of the permutation group $\operatorname{Sym}_{k}$. Point $E \in \mathrm{M}_{\gamma}$ belongs to the stratum corresponding to $\left(\gamma_{1}, \ldots, \gamma_{k}\right)$ if and only if the representation $E$ can be decomposed into a direct sum of $T$-indecomposable subrepresentations $E^{j}, j=1, \ldots, k$ in coordinate subspaces of dimension vectors $\gamma_{j}$.

Then we can use spectral sequences and Thom isomorphisms as in Section 5.2, and obtain an identity between generating series. At this point it is convenient to use cohomology of stacks with compact support. We have a constructible equivalence of ind-Artin stacks

$$
\sqcup_{\gamma \in \mathbb{Z} \geqslant 0}^{I} \mathrm{M}_{\gamma} /\left(\operatorname{Sym}_{\gamma} \ltimes \mathrm{T}_{\gamma}\right) \simeq \operatorname{Sym}\left(\sqcup_{\gamma \in \mathbb{Z}_{\geqslant 0}^{I}-\{0\}} \mathrm{M}_{\gamma}^{T-\text { ind }} /\left(\operatorname{Sym}_{\gamma} \ltimes \mathrm{T}_{\gamma}\right)\right),
$$


where for any ind-Artin stack $X$ we define $\operatorname{Sym}(X):=\sqcup_{k \geqslant 0} X^{k} / \operatorname{Sym}_{k}$. The constructible equivalence implies the identity

$$
\begin{aligned}
& \sum_{\gamma \in \mathbb{Z}_{\geqslant 0}^{I}}\left[\mathbf{H}_{c, \mathrm{~T}_{\gamma}}^{\bullet}\left(\mathrm{M}_{\gamma},-W_{\gamma}\right)^{\mathrm{Sym}_{\gamma}}\right] x^{\gamma} \\
& \quad=\operatorname{Sym}\left(\sum_{\gamma \in \mathbb{Z}_{\geqslant 0}^{I}-\{0\}}\left[\mathbf{H}_{c, \mathrm{~T}_{\gamma}}^{\bullet}\left(\mathrm{M}_{\gamma}^{T-\text { ind }},-W_{\gamma}\right)^{\left.\mathrm{Sym}_{\gamma}\right] x^{\gamma}}\right) .\right.
\end{aligned}
$$

Passing to the dual spaces, and making the change of line variables $x^{\gamma} \mapsto$ $\mathbb{L}^{\operatorname{dim} \mathrm{T}_{\gamma}} x^{\gamma}$, we obtain the following formula (see Section 5.3 for the definition of $\left.\left[\mathcal{H}_{\gamma}\right]\right)$ :

$$
\begin{aligned}
& \sum_{\gamma \in \mathbb{Z}_{\geqslant 0}^{I}}\left[\mathcal{H}_{\gamma}\right] \mathbb{L}^{-\operatorname{dim} \mathrm{M}_{\gamma}} x^{\gamma} \\
& \quad=\operatorname{Sym}\left(\sum _ { \gamma \in \mathbb { Z } _ { \geqslant 0 } ^ { I } - \{ 0 \} } \left[\mathbf{H}_{\mathbf{T}_{\gamma}}^{\bullet}\left(\mathrm{M}_{\gamma}^{T-\text { ind }}, W_{\gamma}\right)^{\left.\left.\operatorname{Sym}_{\gamma}\right] \mathbb{L}^{-\operatorname{dim} \mathrm{M}_{\gamma}} x^{\gamma}\right) .}\right.\right.
\end{aligned}
$$

Notice that for any $\gamma \in \mathbb{Z}_{\geqslant 0}^{I}-\{0\}$ one has an exact sequence of groups

$$
1 \rightarrow \mathbb{G}_{m}^{\operatorname{diag}} \rightarrow \mathrm{T}_{\gamma} \rightarrow \mathrm{PT}_{\gamma} \rightarrow 1
$$

where $\mathbb{G}_{m}^{\text {diag }}$ is the naturally embedded diagonal subgroup of $\mathrm{T}_{\gamma}$, and $\mathrm{PT}_{\gamma}$ is the quotient group. Subgroup $\mathbb{G}_{m}^{\text {diag }}$ acts trivially on $\mathbf{M}_{\gamma}^{T-i n d}$, and the quotient group $\mathrm{PT}_{\gamma}$ acts freely on $\mathrm{M}_{\gamma}^{T-\text { ind }}$. Therefore we have

$$
\mathbf{H}_{\mathrm{T}_{\gamma}}^{\bullet}\left(\mathrm{M}_{\gamma}^{T-\text { ind }}, W_{\gamma}\right)^{\operatorname{Sym}_{\gamma}} \simeq \mathbf{H}^{\bullet}\left(\mathrm{M}_{\gamma}^{T-\text { ind }} / \mathrm{PT}_{\gamma}, W_{\gamma}\right)^{\operatorname{Sym}_{\gamma}} \otimes \mathbf{H}^{\bullet}\left(B \mathbb{G}_{m}^{\text {diag }}\right),
$$

where in RHS we take ordinary cohomology of the quotient variety endowed with a function. Hence we have proved the following result that is parallel to Theorem 6.2 from Section 6.2:

$$
\text { The series } \sum_{\gamma \in \mathbb{Z}_{\geqslant 0}^{I}}\left[\mathcal{H}_{\gamma}\right] \mathbb{L}^{-\operatorname{dim} \mathrm{M}_{\gamma}} x^{\gamma} \text { is admissible. }
$$

\subsection{One-dimensional factorization systems}

There is an operation of tensor product (over $\left(\mathcal{O}\left(\mathbb{A}_{K}^{\gamma}\right)\right)_{\gamma \in \mathbb{Z}_{\geqslant 0}^{I}-\{0\}}$ ) on factorization systems, and moreover the product of two non-singular systems is 
again non-singular. In this section, we will define a class of invertible factorization systems such that underlying sheaves are super line bundles on configuration spaces. These systems form a subgroup of the Picard group of the tensor category of factorization systems.

Let $\mathcal{B}=\left(b_{i j}\right)_{i, j \in I}$ be an integral symmetric matrix. Denote by $\epsilon: \mathbb{Z}^{I} \rightarrow$ $\mathbb{Z} / 2 \mathbb{Z}$ the group homomorphism given by

$$
\epsilon(\gamma):=\sum_{i \in I} b_{i i} \gamma^{i} \bmod 2
$$

By the arguments from Section 2.6 there exists a homomorphism (bilinear form)

$$
\phi: \mathbb{Z}^{I} \otimes \mathbb{Z}^{I} \rightarrow \mathbb{Z} / 2 \mathbb{Z}
$$

such that

$$
\sum_{i, j} b_{i j} \gamma_{1}^{i} \gamma_{2}^{j}+\epsilon\left(\gamma_{1}\right) \epsilon\left(\gamma_{2}\right)+\phi\left(\gamma_{1}, \gamma_{2}\right)+\phi\left(\gamma_{2}, \gamma_{1}\right)=0 \bmod 2, \quad \forall \gamma_{1}, \gamma_{2} \in \mathbb{Z}^{I}
$$

Let us choose such a bilinear form $\phi$. We define a collection of super coherent sheaves $\mathcal{L}_{\gamma}^{\mathcal{B}}$ as trivialized line bundles on $\mathbb{A}_{K}^{\gamma}$ (with generators denoted by $s_{\gamma}$ ) endowed with the parity $\epsilon(\gamma)$. We define the action of $\operatorname{Sym}_{\gamma}$ to be the standard action on $\mathcal{L}_{\gamma}^{\mathcal{B}}=\mathcal{O}_{\mathbb{A}_{K}^{\gamma}}$ via permutation of coordinates. The group $\widetilde{G}$ acts on the canonical generator of $\mathcal{L}_{\gamma}^{\mathcal{B}}$ via the representation $(K(-1 / 2))^{\otimes \sum_{i, j} b_{i j} \gamma^{i} \gamma^{j}}$, where $K(-1 / 2)$ is the square root of the Tate motive.

Next, the multiplication isomorphism on $U_{\gamma_{1}, \gamma_{2}}$ is defined on the standard generators such as follows:

$$
\mu_{\gamma_{1}, \gamma_{2}}: s_{\gamma_{1}} \otimes s_{\gamma_{2}} \mapsto \phi\left(\gamma_{1}, \gamma_{2}\right) \prod_{i, \alpha, j, \beta}\left(x_{i, \alpha}-y_{j, \beta}\right)^{b_{i j}} s_{\gamma_{1}+\gamma_{2}}
$$

Lemma 6.5. The collection $\left(\mathcal{L}_{\gamma}^{\mathcal{B}}\right)$ of equivariant super coherent sheaves together with isomorphisms $\mu_{\gamma_{1}, \gamma_{2}}$ defined above, forms a factorization system. It is a non-singular factorization system if $b_{i j} \geqslant 0$ for all $i, j \in I$.

Proof. The associativity is obvious, the commutativity follows by a straightforward check from the relation between $\left(b_{i j}\right), \epsilon, \phi$.

Similar to Section 2.6, one can show that $\left(\mathcal{L}_{\gamma}^{\mathcal{B}}\right)$ does not depend (up to an isomorphism) on the choice of the bilinear form $\phi$, and moreover the identification can be made canonical. 
In the non-singular case when $b_{i j} \geqslant 0$ for all $i, j \in I$, this factorization system admits a very explicit geometric interpretation. Namely, let us consider a quiver $Q^{\mathcal{B}}$ with the set of vertices $I$ and $b_{i j}$ arrows between vertices $i$ and $j$. We endow this quiver with the non-degenerate quadratic potential

$$
W^{\mathcal{B}}=\sum_{i \neq j} \sum_{1 \leqslant l \leqslant b_{i j}} x_{i, j ; l} x_{i, j ; l}^{*}+\sum_{i \in I} \sum_{1 \leqslant l \leqslant b_{i i}}\left(y_{i ; l}\right)^{2} .
$$

Here $\left\{x_{i, j ; l}\right\}_{1 \leqslant l \leqslant b_{i j}}$ is the set of all arrows in $Q_{\mathcal{B}}$ connecting vertices $i$ and $j \neq i$, and $*$ denotes an involution on arrows reversing orientation. Also $\left\{y_{i ; l}\right\}_{1 \leqslant l \leqslant b_{i i}}$ is the set of loops at the vertex $i$.

Pair $\left(Q^{\mathcal{B}}, W^{\mathcal{B}}\right)$ gives a non-singular factorization system

$$
\mathcal{F}_{\gamma}^{\mathcal{B}}=\mathbf{H}_{\mathbf{T}_{\gamma}}^{\bullet}\left(\mathrm{M}_{\gamma}^{Q^{\mathcal{B}}}, W_{\gamma}^{\mathcal{B}}\right) \otimes \mathbb{T}_{0}^{-\otimes \sum_{i j} b_{i j} \gamma^{i} \gamma^{j}} .
$$

Function $W_{\gamma}^{\mathcal{B}}$ is a non-degenerate quadratic form on the affine space $\mathrm{M}_{\gamma}^{Q^{\mathcal{B}}}$. By purity, we have

$$
\mathbf{H}_{\mathrm{T}_{\gamma}}^{\bullet}\left(\mathrm{M}_{\gamma}^{Q^{\mathcal{B}}}, W_{\gamma}^{\mathcal{B}}\right) \simeq \mathbf{H}^{\bullet}\left(\mathcal{B} \mathrm{T}_{\gamma}\right) \otimes \mathbf{H}^{\bullet}\left(\mathrm{M}_{\gamma}^{Q^{\mathcal{B}}}, W_{\gamma}^{\mathcal{B}}\right)
$$

Let us assume that the cohomology theory $\mathbf{H}^{\bullet}$ does not distinguish quadratic forms of the same ranks (like e.g. EMHS). In this case we can write

$$
\mathbf{H}^{\bullet}\left(\mathrm{M}_{\gamma}^{Q^{\mathcal{B}}}, W_{\gamma}^{\mathcal{B}}\right) \simeq\left(\mathbb{T}^{\otimes 1 / 2}\right)^{\otimes \sum_{i j} b_{i j} \gamma^{i} \gamma^{j}}
$$

where $\mathbb{T}^{\otimes 1 / 2}=\mathbf{H}^{\bullet}\left(\mathbb{A}_{\mathbf{k}}^{1},-z^{2}\right)=\mathbb{T}_{0}^{\otimes 1 / 2}[-1]$. The action of the permutation group $\operatorname{Sym}_{\gamma}$ on one-dimensional space $\mathbf{H}^{\bullet}\left(\mathbf{M}_{\gamma}^{Q^{\mathcal{B}}}, W_{\gamma}^{\mathcal{B}}\right)$ is trivial.

A straightforward check shows that factorizations systems $\left(\mathcal{F}_{\gamma}^{\mathcal{B}}\right)$ and $\left(\mathcal{L}_{\gamma}^{\mathcal{B}}\right)$ are isomorphic.

\subsection{Approximation by large integers and end of the proof of Theorems 6.1 and 6.2}

The statement of Theorem 6.1 is a certain universal identity in $\lambda$-rings, where the coefficients of polynomials $f_{\gamma}$ in the notation of Definition 6.2 are free $\lambda$-variables. First, we will prove it in the case when $b_{i j} \geqslant 0 \forall i, j \in I$. This can be done in a "model-theory fashion", i.e., by using certain universal identities in $\lambda$-rings.

We start with a non-singular $I$-colored factorization system, where $G=$ $G_{\alpha} \times \mathbb{G}_{m, K}$ for some algebraic group $G_{\alpha}$, and $t^{1 / 2}: G \rightarrow \mathbb{G}_{m, K}$ is the projection to the second factor (see Definition 6.5 ). Let $B_{\alpha}$ denotes the $\lambda$-ring 
$K_{0}\left(G_{\alpha}-\bmod \right)$ and $y_{\gamma} \in B_{\alpha}\left[q^{ \pm 1 / 2}\right]$ be a collection of elements parametrized by $\gamma \in \mathbb{Z}_{\geqslant 0}^{I}-\{0\}$. Then there exists a non-singular factorizations system and the corresponding collection $\left(E_{\gamma}\right)_{\gamma \in \mathbb{Z}_{\geqslant 0}^{I}-\{0\}}$ of super representations (see Theorem 6.4) such that $y_{\gamma}=\left[E_{\gamma}\right] \in K_{0}\left(G_{\alpha}-\bmod \right)$. This can be shown by induction by the norm $|\gamma|$ (for any choice of the norm). Namely, we can consider free non-singular system generated by arbitrary $\widetilde{G} \times \operatorname{Sym}_{\gamma}$-equivariant super coherent sheaves on $\mathbb{A}_{K}^{\gamma}$ supported on the main diagonal for $\gamma \neq 0$.

Next, we can twist the factorization system by the one-dimensional system $\left(\mathcal{F}_{\gamma}^{\mathcal{B}}\right)$ (see Section 6.8) and obtain another non-singular factorization system by Theorem 6.3. Then Theorem 6.4 gives a new collection $\left(E_{\gamma}^{\prime}\right)_{\gamma \in \mathbb{Z}_{\geqslant 0}^{I}-\{0\}}$ and the corresponding classes $y_{\gamma}^{\prime} \in B_{\alpha}\left[q^{ \pm 1 / 2}\right]$. By Corollary to Theorem 6.4 we obtain the admissibility property.

In order to prove Theorem 6.1 for general $\lambda$-rings it suffices to have it for infinitely generated free $\lambda$-rings. Those can be "approximated" by $\lambda$-rings of the type $K_{0}\left(G_{\alpha}-\right.$ mod $)$ such as follows. Let us fix a non-zero element $\gamma_{0}$ and number $c \geqslant 0$ such that

$$
y_{\gamma}=\sum_{|i| \leqslant c} y_{\gamma, i} q^{i / 2}
$$

for all $|\gamma| \leqslant\left|\gamma_{0}\right|$. Then $y_{\gamma_{0}}$ is a universal $\lambda$-polynomial in variables $y_{\gamma, i},|\gamma| \leqslant$ $\left|\gamma_{0}\right|,|i| \leqslant c$ and $\frac{1}{1-q}, q^{ \pm 1 / 2}$. A priori it belongs to $B_{\alpha}\left(\left(q^{1 / 2}\right)\right)$. The fact that $y_{\gamma_{0}}$ belongs to $B_{\alpha}\left[q^{ \pm 1 / 2}\right]$ is equivalent to a (finite) collection of equations on $y_{\gamma, i}$. We would like to treat $y_{\gamma, i}$ as free $\lambda$-variables. Although this is not the case, we can find a collection of algebraic groups $\left(G_{\alpha}\right)$ and elements $y_{\gamma, i}^{(\alpha)} \in$ $K_{0}\left(G_{\alpha}-\right.$ mod $)$ such that the kernels of the homomorphisms of the free $\lambda$-ring generated by the "universal" symbols $y_{\gamma, i}^{\text {univ }}$ to $K_{0}\left(G_{\alpha}-\bmod \right), y_{\gamma, i}^{\text {univ }} \mapsto y_{\gamma, i}^{(\alpha)}$ are the ideals with the trivial intersection over the set of indices $\alpha$. For example, one can take as $G_{\alpha}$ the product of finitely many copies of $G L(N)$ for large $N$. This finishes the proof of Theorem 6.1 for $b_{i j} \geqslant 0$.

Let $\mathcal{B}=\left(b_{i j}\right)$ be a matrix with integer but not necessarily non-negative coefficients. For each $n \in \mathbb{Z}$ we consider the matrix $\mathcal{B}^{(n)}$ with entries

$$
b_{i j}^{(n)}:=b_{i j}+n, n \in \mathbb{Z} .
$$

For sufficiently large $n$ all entries are non-negative, and hence we have admissibility by the above considerations. For a given $\gamma \neq 0$ let us consider the dependence of $\left[E_{\gamma}\right]$ on $n$. This $\lambda$-polynomial satisfies the following Proposition. 
Proposition 6.5. Let $B$ be a $\lambda$-ring, and $P$ be a $\lambda$-polynomial with coefficients in $B$ in three variables $x, y, z$, where $y, z$ are invertible line elements. Suppose that for any sufficiently large integer $n \gg 0$ the value

$$
P\left(\frac{1}{1-q}, q^{1 / 2},\left(-q^{1 / 2}\right)^{n}\right) \in B\left(\left(q^{1 / 2}\right)\right)
$$

is a Laurent polynomial in the line variable $q^{1 / 2}$. Then the same is true for any integer $n \in \mathbb{Z}$.

Proof. First, we have an identity in the $\lambda$-ring $\mathbb{Z}((q))$ :

$$
\lambda^{k}\left(\frac{1}{1-q}\right)=\frac{q^{k(k-1) / 2}}{(1-q) \ldots\left(1-q^{k}\right)}, \forall k \geqslant 0
$$

Hence, the evaluation $P\left(\frac{1}{1-q}, q^{1 / 2},\left(-q^{1 / 2}\right)^{n}\right)$ can be identified with

$$
\frac{1}{\left(1-q^{A}\right)^{D}} \cdot \sum_{i, j:|i|+|j|<N} c_{i j}\left(-q^{1 / 2}\right)^{i+n j}
$$

for some integers $A, D, N \geqslant 1$ and elements $c_{i j} \in B$. Our assumption means that for any $n \geqslant 0$ the above series is a Laurent polynomial, i.e., its coefficients in sufficiently large powers of $-q^{1 / 2}$ vanish. $\mathbb{Z}_{\geqslant 0}^{I}$-grade.

Let us fix two residues $a_{1}, a_{2} \in \mathbb{Z} / A \mathbb{Z}$. It is easy to see that the coefficient of $\left(-q^{1 / 2}\right)^{k}$ for $k \gg 1$ and $k=a_{1}(\bmod A), n=a_{2}(\bmod A)$ can be written as

$$
\sum_{i, j} c_{i j} F_{i, j, a_{1}, a_{2}}(k, n)
$$

where $F_{i, j, a_{1}, a_{2}}$ is a polynomial in two variables depending on residues $\left(a_{1}, a_{2}\right)$ modulo $A$, with integer values at $(k, n) \in \mathbb{Z}^{2}$. Consider the additive subgroup $\Gamma$ of $B$ generated by $\left(c_{i j}\right)$. It is a finitely generated abelian group. Pick any additive functional $\Gamma \rightarrow \mathbb{Z} / M \mathbb{Z}$ for some $M \geqslant 0$. Then we use the following obvious statement.

Lemma 6.6. Let $f: \mathbb{Z} \times \mathbb{Z} \rightarrow \mathbb{Z}$ be a $\mathbb{Z}$-valued polynomial. If $f(x, y)=0$ $(\bmod M)$ for all $x \in \mathbb{Z}$ and all $y \gg 1$ then $f(x, y)=0(\bmod M)$ for all $(x, y) \in \mathbb{Z}^{2}$.

Applying the above Lemma we obtain the proof of the Proposition. 
Returning to the proof of Theorem 6.1 we apply the above Proposition to the $\lambda$-polynomial $\left[E_{\gamma}\right]$ as a function of $n$ and conclude that it belongs to $B\left[q^{ \pm 1 / 2}\right]$ for $n=0$. This finishes the proof of Theorem 6.1 .

Theorem 6.2 now follows from Theorems $6.1,6.3$ and 6.4 .

\section{Critical COHA}

\subsection{Description of results}

Let $R$ be a smooth $I$-bigraded algebra over a field $\mathbf{k}$, endowed with a bilinear form $\chi_{R}$ on $\mathbb{Z}^{I}$ compatible with the Euler characteristic, and a potential $W \in R /[R, R]$, as in Section 4.7. Also, suppose that we are given an additional data, consisting of a collection of $\mathrm{G}_{\gamma}$-invariant closed subsets $\mathrm{M}_{\gamma}^{\text {sp }} \subset \mathrm{M}_{\gamma}$ for all $\gamma \in \mathbb{Z}_{\geqslant 0}^{I}$ (superscript sp means "special") satisfying the following conditions:

- for any $\gamma$ we have $\mathrm{M}_{\gamma}^{\mathrm{sp}} \subset \operatorname{Crit}\left(W_{\gamma}\right)$, i.e., 1 -form $d W_{\gamma}$ vanishes at $\mathrm{M}_{\gamma}^{\mathrm{sp}}$;

- for any short exact sequence $0 \rightarrow E_{1} \rightarrow E \rightarrow E_{2} \rightarrow 0$ of representations of $\overline{\mathbf{k}} \otimes_{\mathbf{k}} R$ with dimension vectors $\gamma_{1}, \gamma:=\gamma_{1}+\gamma_{2}, \gamma_{2}$ correspondingly, such that all $E_{1}, E_{2}, E$ are critical points of the potential,the representation $E$ belongs to $\mathrm{M}_{\gamma}^{\mathrm{sp}}$ if and only if both representations $E_{1}, E_{2}$ belong to $\mathrm{M}_{\gamma_{1}}^{\mathrm{sp}}, \mathrm{M}_{\gamma_{2}}^{\mathrm{sp}}$, respectively.

The last condition implies that the collection of representations from $\mathrm{M}_{\gamma}^{\mathrm{sp}}(\overline{\mathbf{k}})$ for all $\gamma \in \mathbb{Z}_{\geqslant 0}^{I}$ form an abelian category, which is a Serre subcategory of the abelian category $\operatorname{Crit}(W)(\overline{\mathbf{k}}):=\sqcup_{\gamma} \operatorname{Crit}\left(W_{\gamma}\right)(\overline{\mathbf{k}})$, which is itself a full subcategory of $\overline{\mathbf{k}} \otimes_{\mathbf{k}} R-\bmod$. For example, one can always make the maximal choice

$$
\mathrm{M}_{\gamma}^{\mathrm{sp}}:=\operatorname{Crit}\left(W_{\gamma}\right) \forall \gamma \in \mathbb{Z}_{\geqslant 0}^{I}
$$

We can construct more examples such as follows. Pick an arbitrary subset $N \subset R$ and define $\mathrm{M}_{\gamma}^{\text {sp }}$ as the set of representations belonging to $\operatorname{Crit}\left(W_{\gamma}\right)$ for which all elements $n \in N$ act as nilpotent operators.

Assume that $\mathbf{k}=\mathbb{C}$. We will define the critical $C O H A$ as

$$
\mathcal{H}=\oplus_{\gamma \in \mathbb{Z}_{\geqslant 0}^{I}} \mathcal{H}_{\gamma}
$$

where

$$
\mathcal{H}_{\gamma}:=\bigoplus_{z \in \mathbb{C}}\left(H_{\mathrm{G}_{\gamma}, c}^{\bullet}\left(\mathrm{M}_{\gamma}^{\mathrm{sp}} \cap W_{\gamma}^{(-1)}(z), \phi_{W_{\gamma}-z} \mathbb{Q}_{\mathrm{M}_{\gamma}}\right)\right)^{\vee} \otimes \mathbb{T}^{\otimes \operatorname{dim} M_{\gamma} / G_{\gamma}}
$$


Here we use equivariant cohomology with compact support with coefficients in the sheaf of vanishing cycles (see below).

We will define a structure of EMHS of special type (called monodromic mixed Hodge structure) with Betti realization $\mathcal{H}_{\gamma}$, and a twisted associative product. For the critical COHA in Tannakian category EMHS, there are analogs of results from Sections 5 and 6 . In particular, for any stability condition we obtain a factorization of the motivic DT-series, and all motivic DT-series associated with sectors are admissible. The proof of the factorization property for a chosen stability condition is much more complicated than in the case of the exponential cohomology. It is based on certain "integral identity" involving vanishing cycles and conjectured in our earlier work [33] (also we proposed there a sketch of the proof). The reason for the complication is the lack of Thom isomorphism for vanishing cycles. In Section 7.8, we will give a complete proof of the identity.

Under certain assumptions one can show that the critical COHA has the same motivic DT-series as the one defined for rapid decay cohomology. In particular, we can use this fact in the case of quiver $Q_{1}$ with polynomial potential. Also, "critical" DT-series introduced below matches those introduced in [33] in the framework of ind-constructible three-dimensional Calabi-Yau categories. We should mention that the idea of defining Donaldson-Thomas invariants using compactly supported cohomology of the vanishing cycle complex appeared first in [13] and also was implicit in $[33]$.

The generality of our construction is still not satisfactory for all applications. One needs a minor generalization of the theory of vanishing cycles to formal schemes and stacks, which is still absent in the existing literature. In the ideal picture, our theory will be applicable to a large class of threedimensional Calabi-Yau categories, including those associated with quivers with formal potentials, e.g., the cluster ones.

\subsection{Vanishing cycles in the analytic case}

Let us first recall basic facts about vanishing cycles for constructible sheaves in analytic geometry (see e.g. [51]). If $X$ is a complex analytic space endowed with a holomorphic function $f: X \rightarrow \mathbb{C}$, then we have the functor $\psi_{f}$ of nearby cycles from $D_{c}^{b}(X)$, the derived category of complexes of sheaves on $X$ constructible with respect to a complex analytic stratification, into a similar one for $X_{0}:=f^{-1}(0)$, given (in the obvious notation) by

$$
\psi_{f}:=\left(X_{0} \rightarrow X\right)^{*} \circ\left(X_{+} \rightarrow X\right)_{*} \circ\left(X_{+} \rightarrow X\right)^{*}, \quad X_{+}:=f^{-1}\left(\mathbb{R}_{>0}\right) \subset X
$$


Also we have the functor $\phi_{f}$ of vanishing cycles given by the cone of the adjunction

$$
\phi_{f} \mathcal{F} \simeq \operatorname{Cone}\left(\left(X_{0} \rightarrow X\right)^{*} \mathcal{F} \rightarrow \psi_{f} \mathcal{F}\right) .
$$

The functor of vanishing cycles commutes with the direct image for proper morphisms $\pi: X_{1} \rightarrow X_{2}$ for varieties with functions $f_{1}, f_{2}$ such that $f_{1}=\pi^{*} f_{2}$,

$$
\phi_{f_{2}} \circ \pi_{*} \simeq \pi_{*} \circ \phi_{f_{1}} .
$$

Thom-Sebastiani theorem for sheaves (see e.g. [42,51]) says that the shifted functor of vanishing cycles commutes with the external products. In other words, if $X=X^{(1)} \times X^{(2)}$ and $f=f^{(1)} \boxplus f^{(2)}$ for $f^{(i)} \in \mathcal{O}\left(X^{(i)}\right), i=$ 1,2 and

$$
\mathcal{F}=\mathcal{F}^{(1)} \otimes \mathcal{F}^{(2)}:=\operatorname{pr}_{X \rightarrow X^{(1)}}^{*}\left(\mathcal{F}^{(1)}\right) \otimes \operatorname{pr}_{X \rightarrow X^{(2)}}^{*}\left(\mathcal{F}^{(2)}\right)
$$

for $\mathcal{F}^{(i)} \in D_{c}^{b}\left(X^{(i)}\right), i=1,2$, then

$$
\left(X_{0}^{(1)} \times X_{0}^{(2)} \rightarrow X_{0}\right)^{*} \phi_{f}[-1] \mathcal{F} \simeq \phi_{f^{(1)}}[-1] \mathcal{F}^{(1)} \otimes \phi_{f^{(2)}}[-1] \mathcal{F}^{(2)},
$$

where $X_{0}^{(1)}, X_{0}^{(2)}$ and $X_{0}$ are zero loci of functions $f^{(1)}, f^{(2)}$ and $f$, respectively.

\subsection{Relation with the rapid decay cohomology}

For a large class of functions, one can relate rapid decay cohomology and vanishing cycles. Let $X$ be a complex algebraic variety. For a regular function, $f \in \mathcal{O}(X)$ considered as a map $X \rightarrow \mathbb{A}_{\mathbb{C}}^{1}$, we define its bifurcation set $\operatorname{Bif}(f) \subset \mathbb{C}$ as the set of points over which $f$ is not a locally trivial fibration (in the analytic topology). The set $\operatorname{Bif}(f)$ is finite, containing singularities of the direct image $f_{*} \mathbb{Z}_{X(\mathbb{C})}$. Also, for smooth $X$ the set $\operatorname{Bif}(f)$ contains the set of critical values of $f$.

Let us choose an isotopy class of a collection of disjoint paths from $-\infty$ to points of $\operatorname{Bif}(f)$. Then we obtain a canonical isomorphism

$$
H^{\bullet}(X, f)=H^{\bullet}\left(X, f^{-1}(-c)\right) \simeq \bigoplus_{z \in \operatorname{Bif}(f)} H^{\bullet}\left(f^{-1}\left(B_{\delta}(z)\right), f^{-1}(z-\delta)\right)
$$

for sufficiently small real $0<\delta \ll 1$ and sufficiently large real $c \gg 0$. Here $B_{\delta}(z):=\{w \in \mathbb{C}|| z-w \mid \leqslant \delta\}$. Indeed, the pointed topological space 
$X(\mathbb{C}) / f^{-1}(-c)$ is homotopy equivalent to the wedge sum (coproduct) of pointed spaces

$$
\bigvee_{z \in \operatorname{Bif}(f)} f^{-1}\left(B_{\delta}(z)\right) / f^{-1}(z-\delta),
$$

with the homotopy equivalence depending on the collection of disjoint paths (up to isotopy).

Definition 7.1. A function $f$ on smooth algebraic variety $X / \mathbb{C}$ is called topologically isotrivial at infinity if there exists a $C^{\infty}$-manifold with boundary $U \subset X(\mathbb{C}), \operatorname{dim}_{\mathbb{R}} U=2 \operatorname{dim} X$ such that $f_{\mid U}: U \rightarrow \mathbb{C}$ is proper, and a homeomorphism

$$
h: \partial U \times[0,+\infty) \simeq X(\mathbb{C})-\operatorname{int} U, \quad h(x, 0)=x \text { for } x \in \partial U,
$$

such that $f(h(x, t))=f(x)$ for any $x \in \partial U$.

In particular, if $f: X \rightarrow \mathbb{C}$ is a proper map for smooth $X$, then it is topologically isotrivial at infinity. We were not able to find a discussion of this property in the literature. The closest analog is a strictly stronger property called $M$-tame (see [44]). It is easy to see that for a function $f$ topologically isotrivial at infinity, the bifurcation set coincides with the set of critical values, and for any $z \in \operatorname{Bif}(f)$ one has a canonical isomorphism

$$
H^{\bullet}\left(f^{-1}(z), \phi_{-f+z} \mathbb{Z}_{X}[-1]\right) \simeq H^{\bullet}\left(f^{-1}\left(B_{\delta}(z)\right), f^{-1}(z-\delta)\right)
$$

for $0<\delta \ll 1$. Hence, we see that for such $f$ the direct sum over critical values

$$
\bigoplus_{z \in \mathbb{C}} H^{\bullet}\left(f^{-1}(z), \phi_{-f+z} \mathbb{Z}_{X}[-1]\right)
$$

is isomorphic to $H^{\bullet}(X, f)$. The isomorphism is not canonical, it depends on a choice of an isotopy class of a collection of disjoint paths from $-\infty$ to points of $\operatorname{Bif}(f)$, as before.

Also we have a (non-canonical) isomorphism between cohomology with compact support for a topologically isotrivial at infinity function $f$

$$
H_{\mathrm{c}}^{\bullet}(X, f) \simeq \bigoplus_{z \in \mathbb{C}} H_{\mathrm{c}}^{\bullet}\left(f^{-1}(z), \phi_{-f+z} \mathbb{Z}_{X}[-1]\right) .
$$

This follows by Verdier duality from the previous considerations.

Here is an application promised in Section 4.7. Let $W \in \mathbb{C}[x]$ be a polynomial of degree $N \geqslant 3$ such that $W^{\prime}$ has $N-1$ distinct roots $r_{1}, \ldots, r_{N-1} \in \mathbb{C}$. 
We can consider $W$ as a cyclic polynomial in $\mathbb{C} Q_{1}$. Hence for any $n \geqslant 0$, we get function $W_{n}$ on $\operatorname{Mat}(n \times n, \mathbb{C})$ given by $W_{n}(X)=\operatorname{Tr} W(X)$. One can show that for an appropriate $C^{\infty}$ function $F: \mathbb{C} \rightarrow \mathbb{R}_{>0}$ and any $n$, the submanifold $U_{n} \subset \operatorname{Mat}(n \times n, \mathbb{C})$ given by the inequality

$$
\operatorname{Tr}\left(X X^{\dagger}\right) \leqslant F(\operatorname{Tr}(W(X)))
$$

satisfies an $U(n)$-equivariant version of the conditions from the definition of topological isotriviality at infinity. Thus, implies that one can identify equivariant rapid decay cohomology with the equivariant cohomology of vanishing cycles. The latter is easy to compute.

The set of critical points of the potential $W_{n}$ consists of operators $x \in$ $\operatorname{Mat}(n \times n, \mathbb{C})$ satisfying the equation

$$
W^{\prime}(x)=0 .
$$

This means that $x$ has eigenvalues $r_{1}, \ldots, r_{N-1}$ with some multiplicities, and no non-trivial Jordan blocks. Therefore the space $\mathbb{C}^{n}$ splits into the direct sum $\oplus_{i=1}^{N-1} V_{i}$ of eigenspaces. We conclude that the set of critical points of $W_{n}$ is the disjoint union over all ordered partitions

$$
n_{1}+\cdots+n_{N-1}, \quad n_{i} \geqslant 0
$$

of components corresponding to such decompositions with $\operatorname{dim} V_{i}=n_{i}$.

Potential $W_{n}$ has Bott-Morse singularity at any critical point, i.e., locally (in analytic topology) it is the Thom-Sebastiani sum $\boxplus$ of a constant function and of a quadratic form.

The rank of the second derivative $W_{n}^{\prime \prime}$ at the component corresponding to the decomposition $n_{1}+\cdots+n_{N-1}$, is equal to $\sum_{i} n_{i}^{2}$. Also, this component is a homogeneous space $\mathrm{GL}(n, \mathbb{C}) / \prod_{i} \mathrm{GL}\left(n_{i}, \mathbb{C}\right)$. One can check that the sheaf of vanishing cycles (shifted by $[-1]$ ) in our case is the constant sheaf $\underline{\mathbb{Z}}$ shifted by $\left[-\sum_{i} n_{i}^{2}\right]$. Therefore, we conclude that

$$
\mathcal{H}_{n} \simeq \bigoplus_{\substack{n_{1}, \ldots, n_{N-1} \geqslant 0 \\ n_{1}+\cdots+n_{N-1}}} \prod_{i=1}^{N-1} H^{\bullet}\left(\operatorname{BGL}\left(n_{i}, \mathbb{C}\right)\right) .
$$

Then $\mathcal{H}=\bigoplus_{n \geqslant 0} \mathcal{H}_{n}$ is isomorphic as a bigraded space to

$$
\left(\bigoplus_{m \geqslant 0} H^{\bullet}(\operatorname{BGL}(m, \mathbb{C}))\right)^{\otimes(N-1)} .
$$


It looks plausible that $\mathcal{H}$ considered as an algebra (and not only as a bigraded vector space), is also isomorphic to the $(N-1)$-st tensor power of the exterior algebra corresponding to the case $N=2$. Also it looks plausible that the condition on $W$ to have simple critical points can be dropped.

\subsection{Vanishing cycles and monodromic Hodge structures}

The definition of vanishing cycles given in the analytic case is not algebrogeometric. To our knowledge there is no satisfactory general geometric approach as well as a proof of Thom-Sebastiani theorem. There are individual theories in the étale case (see [52]), and for mixed Hodge modules (see [49]). In what follows we will work over $\mathbf{k}=\mathbb{C}$ and use mixed Hodge modules, although all arguments hold also for étale constructible sheaves as well.

Cohomology groups of sheaves of vanishing cycles carry a natural mixed Hodge structure. In fact, they should be considered as EMHS of special type, which we will call monodromic. The origin of the name is explained by the fact that there is a natural action of monodromy operator on $\phi_{f} \mathcal{F}$. The monodromy comes from the parallel transport around 0 for locally constant family of sheaves $\phi_{\lambda f} \mathcal{F}$ depending on a parameter $\lambda \in \mathbb{C}^{*}$.

Definition 7.2. Tannakian category of 'monodromic mixed Hodge structures' MMHS is the full Tannakian subcategory of EMHS consisting of objects unramified on $\mathbb{C}^{*} \subset \mathbb{C}$.

As an abelian category MMHS is naturally equivalent to the category of unramified mixed Hodge modules on $\mathbb{G}_{m, \mathbb{C}}$, i.e., admissible variations of mixed Hodge structures on $\mathbb{C}^{*}$. The correspondence (shifted by 1 ) is given in one way by the restriction from $\mathbb{C}=\mathbb{A}^{1}(\mathbb{C})$ to $\mathbb{C}^{*}$, and in another way by the direct image with compact supports.

Tensor product on Tannakian category MMHS does not coincide with the tensor product of corresponding variations of mixed Hodge structures on on $\mathbb{C}^{*}$, but for the underlying constructible sheaves of $\mathbb{Q}$-vector spaces there is an isomorphism of two tensor products.

Definition 7.3. Let $X / \mathbb{C}$ be an algebraic variety endowed with a function $f \in \mathcal{O}(X)$, and a locally closed subset $X^{\mathrm{sp}} \subset X_{0}:=f^{-1}(0)$. The 'critical cohomology with compact support' is defined as the cohomology of an object of $D^{b}(\mathrm{MMHS}) \subset D^{b}\left(\mathrm{MHM}_{\mathbb{A}_{\mathbb{C}}^{1}}\right)$, given by

$$
\left(\mathbb{G}_{m} \rightarrow \mathbb{A}_{\mathbb{C}}^{1}\right) !\left(X^{\mathrm{sp}} \times \mathbb{G}_{m} \rightarrow \mathbb{G}_{m}\right) !\left(\left(X^{\mathrm{sp}} \times \mathbb{G}_{m}\right) \rightarrow\left(X_{0} \times \mathbb{G}_{m}\right)\right)^{*} \phi_{\frac{f}{u}} \mathbb{Q}_{X \times \mathbb{G}_{m}}
$$


where affine line $\mathbb{A}_{\mathbb{C}}^{1}$ is endowed with coordinate $u$. We will denote it by $H_{c, \mathrm{MMHS}}^{\bullet, \mathrm{crit}}\left(X^{\mathrm{sp}}, f\right)$.

The above definition becomes very transparent when one applies the Betti realization functor at $u=1$. Then the critical cohomology with compact support turns into a space

$$
H_{\mathrm{c}}^{\bullet}\left(X^{\mathrm{sp}},\left(X^{\mathrm{sp}} \rightarrow X_{0}\right)^{*} \phi_{f}\left(\mathbb{Q}_{X}\right)\right)
$$

endowed with the action of the monodromy operator.

The reader should notice that (as we already pointed out in Section 4.5) the critical cohomology depends on the ambient space $X$ and the corresponding function $f_{X}$. This is quite different from the case of rapid decay cohomology.

There is a version of the Thom-Sebastiani theorem in the setting of mixed Hodge modules (Saito [50]). It implies in particular an isomorphism

$$
\begin{aligned}
& H_{\mathrm{c}, \mathrm{MMHS}}^{\bullet}\left(X^{(1), \mathrm{sp}}, f^{(1)}\right) \otimes H_{\mathrm{c}, \mathrm{MMHS}}^{\bullet, \mathrm{Mrit}}\left(X^{(2), \mathrm{sp}}, f^{(2)}\right) \\
& \quad \simeq H_{\mathrm{c}, \mathrm{MMHHS}}^{\bullet}\left(X^{(1), \mathrm{sp}} \times X^{(2), \mathrm{sp}}, f\right)
\end{aligned}
$$

where $X=X^{(1)} \times X^{(2)}, f=f^{(1)} \boxplus f^{(2)}, f^{(i)} \in \mathcal{O}\left(X^{(i)}\right), i=1,2$ and the tensor product is the additive convolution $*_{+}$.

Let $\mathbf{k}$ be a field, char $\mathbf{k}=0$ and $\mathbf{H}^{\bullet}$ be one of the standard cohomology theories, namely étale cohomology, Betti cohomology, or mixed Hodge modules. Then we can define similarly to what has been done above, monodromic exponential mixed motives $\mathbf{H}_{\mathrm{c}}^{\bullet}$,crit $\left(X^{\mathrm{sp}}, f\right)$ for triples $\left(X, f, X^{\mathrm{sp}}\right)$ defined over k. It is expected that the Thom-Sebastiani theorem holds also in the étale case (P. Deligne, private communication $)^{13}$. In what follows, we will use the notation $\mathbf{H}_{\mathbf{c}}^{\bullet}$,crit assuming the Thom-Sebastiani theorem. The cautious reader can always replace it by $H_{\mathrm{c}, \mathrm{MMHS}}^{\bullet}$.

Another unsatisfactory issue which we have already mentioned in Section 7.1 is that vanishing cycles are not yet defined for formal functions. Ideally, we would like to have a definition of the sheaf of vanishing cycles " $\phi_{f} \mathbb{Q}_{X}$ " associated with any smooth formal scheme $\mathfrak{X} / \mathbf{k}$ endowed with a function $f \in \mathcal{O}(\mathfrak{X})$. This should be a constructible sheaf on the ordinary reduced scheme $f^{-1}(0) \cap \mathfrak{X}_{\text {red }}$, where $\mathfrak{X}_{\text {red }}$ is obtained from $\mathfrak{X}$ by killing all topologically nilpotent elements. In the case when $\mathfrak{X}$ is a completion of an

${ }^{13}$ After this pape was finished, a proof of the Thom-Sebastiani theorem in the case of positive characteristic was announced by Lei Fu; see [20]. 
ordinary scheme $X$ along a closed subset $X^{(0)} \subset X$, and $f$ is a restriction of $f_{X} \in \mathcal{O}(X)$, the sheaf " $\phi_{f} \mathbb{Q}_{X}$ " should be canonically isomorphic to the restriction of $\phi_{f_{X}} \mathbb{Q}_{X}$ to $f_{X}^{-1}(0) \cap X^{(0)}$. Also we would like to have equivariant cohomology with compact support, and more generally cohomology of stacks.

There is a great variety of partial results which leave no doubts that such a theory should exist, see $[6,7,45,27]$. For a geometric theory formulated in terms of the Grothendieck group of varieties, one has good indications that the corresponding version of the Thom-Sebastiani theorem holds; see [11]. Also, in the same spirit, see [53] for appropriate results on the equivariant resolution of singularities in the formal setting.

\subsection{Rapid decay cohomology and monodromic Hodge structures}

Finally, we would like to return to the comparison with rapid decay cohomology, already discussed in Section 7.3, but this time at the level of Hodge structures. There is an exact faithful tensor functor $F:$ EMHS $\rightarrow$ $\mathrm{MHM}^{\mathbb{C}-\text { grad }}$, where MHM${ }^{\mathbb{C}-\text { grad }}$ consists of finite direct sums $\oplus_{z_{i} \in \mathbb{C}} M_{z_{i}}$ of monodromic mixed Hogde modules. The functor $F$ assigns to $M \in O b$ (EMHS) the direct sum of the corresponding monodromic mixed Hodge modules over the finite set $S:=\left\{z_{1}, \ldots, z_{n}\right\}$ of singular points of $M$. In order to define the summands $M_{z_{i}}$ we consider $M$ as an object of $\mathrm{MHM}_{\mathbb{A}_{\mathbb{C}}^{1}}$. Each $M_{z_{i}}$ is given by the formula similar to the one from Definition 7.3. Namely,

$$
M_{z_{i}}=\left(\left\{z_{i}\right\} \times \mathbb{G}_{m, \mathbb{C}} \rightarrow \mathbb{A}_{\mathbb{C}}^{1}\right) ! \phi_{\frac{z-z_{i}}{u}}\left(\mathbb{A}_{\mathbb{C}}^{1} \times \mathbb{G}_{m, \mathbb{C}} \rightarrow \mathbb{A}_{\mathbb{C}}^{1}\right)^{*} M
$$

where the map $\left\{z_{i}\right\} \times \mathbb{G}_{m, \mathbb{C}} \rightarrow \mathbb{A}_{\mathbb{C}}^{1}$ is given by $\left(z_{i}, u\right) \mapsto u$.

There is an analog of the functor $F$ in the framework of non-commutative Hodge structures (see [30]). Namely, with a nc Hodge structure of exponential type one can associate a $\mathbb{C}$-graded nc Hodge structure with regular singularities by forgetting the gluing data (cf. Theorem 2.35 from loc. cit.). In terms of $D$-modules the situation is described as forgetting the Stokes data, and hence passing to the formal classification of $D$-modules ${ }^{14}$. Notice that the functor $F$ preserves the weight filtration, and hence it preserves the Serre polynomial. In the case of the function $f$ which is isotrivial at infinity

\footnotetext{
${ }^{14}$ Similar story appears in matrix integrals. In that case the critical cohomology corresponds to the formal expansion. For 1-matrix integrals filling fractions play a role of the dimension of a representation of a quiver.
} 
we have:

$$
F\left(H_{\mathrm{c}, \mathrm{EMHS}}(X, f)^{\bullet}\right) \simeq \bigoplus_{z \in B i f(f)} H_{\mathrm{c}, \mathrm{MMHS}}^{\bullet, \mathrm{crit}}\left(f^{-1}(z), f-z\right)
$$

\subsection{Definition of the critical COHA}

Let us assume that we are in the situation described in Section 7.1, i.e., given smooth $I$-bigraded algebra $R / \mathbf{k}$, a bilinear form $\chi_{R}$, a potential $W$, and collection of subsets $\mathrm{M}_{\gamma}^{\mathrm{sp}}$ satisfying the conditions from Section 7.1. The cohomology space $\mathcal{H}_{\gamma}$ is a $\mathbb{Z}$-graded object of the category of monodromic exponential mixed $\mathbf{H}$-motives defined as

$$
\mathcal{H}_{\gamma}:=D\left(\mathbf{H}_{\mathrm{c}, \mathrm{G}_{\gamma}}^{\bullet, \operatorname{cit}}\left(\mathrm{M}_{\gamma}^{\mathrm{sp}}, W_{\gamma}\right)\right) \otimes \mathbb{T}^{\otimes \operatorname{dim} \mathrm{M}_{\gamma} / \mathrm{G}_{\gamma}} .
$$

In the above formula, we define the equivariant cohomology with compact support as the inductive limit, using finite-dimensional approximations to $\mathrm{BG}_{\gamma}$ as in Section 4.5, and $D$ is duality in the ind-completion of the category of monodromic exponential mixed $\mathbf{H}$-motives. In what follows in order to alleviate the notation we assume that potentials $W_{\gamma}$ have only one critical value 0 . The general case is similar.

We have to define the (twisted) multiplication. For any $\gamma_{1}, \gamma_{2} \in \mathbb{Z}_{\geqslant 0}^{I}$, $\gamma:=\gamma_{1}+\gamma_{2}$ we define the dual to the component $m_{\gamma_{1}, \gamma_{2}}$ of the product

$$
\begin{aligned}
m_{\gamma_{1}, \gamma_{2}}^{\vee}: & \mathbf{H}_{c, \mathbf{G}_{\gamma}}^{\bullet, c r i t}\left(\mathrm{M}_{\gamma}^{\mathrm{sp}}, W_{\gamma}\right) \rightarrow \mathbf{H}_{c, \mathbf{G}_{\gamma_{1}}}^{\bullet, c r i t}\left(\mathrm{M}_{\gamma_{1}}^{\mathrm{sp}}, W_{\gamma_{1}}\right) \otimes \mathbf{H}_{c, \mathrm{G}_{\gamma_{2}}}^{\bullet, c r i t}\left(\mathrm{M}_{\gamma_{2}}^{\mathrm{sp}}, W_{\gamma_{2}}\right) \\
& \otimes \mathbb{T}^{\otimes\left(-\chi_{R}\left(\gamma_{2}, \gamma_{1}\right)\right)}
\end{aligned}
$$

as the composition of the following homomorphisms of groups:

- $\mathbf{H}_{\mathrm{c}, \mathrm{G}_{\gamma}}^{\bullet, \text {,rit }}\left(\mathrm{M}_{\gamma}^{\mathrm{sp}}, W_{\gamma}\right) \rightarrow \mathbf{H}_{\mathrm{c}, \mathrm{G}_{\gamma_{1}, \gamma_{2}}}^{\bullet, \text { crit }}\left(\mathrm{M}_{\gamma}^{\mathrm{sp}}, W_{\gamma}\right)$, which is the pull-back associated with the embedding of groups $\mathrm{G}_{\gamma_{1}, \gamma_{2}} \rightarrow \mathrm{G}_{\gamma}$ with proper quotient.

- $\mathbf{H}_{\mathrm{c}, \mathrm{G}_{\gamma_{1}, \gamma_{2}}}^{\bullet, \text { crit }}\left(\mathrm{M}_{\gamma}^{\mathrm{sp}}, W_{\gamma}\right) \rightarrow \mathbf{H}_{\mathrm{c}, \mathrm{G}_{\gamma_{1}, \gamma_{2}}}^{\bullet, \text { crit }}\left(\mathrm{M}_{\gamma_{1}, \gamma_{2}}^{\mathrm{sp}}, W_{\gamma}\right), \quad$ where $\quad \mathrm{M}_{\gamma_{1}, \gamma_{2}}^{\mathrm{sp}}:=\mathrm{M}_{\gamma}^{\mathrm{sp}} \cap$ $\mathrm{M}_{\gamma_{1}, \gamma_{2}}$, also given by the pullback for the closed embedding $\mathrm{M}_{\gamma_{1}, \gamma_{2}}^{\mathrm{sp}} \hookrightarrow$ $\mathrm{M}_{\gamma}^{\mathrm{sp}}$.

- $\mathbf{H}_{\mathrm{c}, \mathrm{G}_{\gamma_{1}, \gamma_{2}}}^{\bullet, \text { crit }}\left(\mathrm{M}_{\gamma_{1}, \gamma_{2}}^{\mathrm{sp}}, W_{\gamma}\right) \simeq \mathbf{H}_{\mathrm{c}, \mathrm{G}_{\gamma_{1}, \gamma_{2}}}^{\bullet, \text { crit }}\left(\widetilde{\mathrm{M}}_{\gamma_{1}, \gamma_{2}}^{\mathrm{sp}}, W_{\gamma}\right)$, where $\tilde{\mathrm{M}}_{\gamma_{1}, \gamma_{2}}^{\mathrm{sp}} \subset \mathrm{M}_{\gamma_{1}, \gamma_{2}}$ is the pullback of $\mathrm{M}_{\gamma_{1}}^{\mathrm{sp}} \times \mathrm{M}_{\gamma_{2}}^{\mathrm{sp}}$ under the projection $\mathrm{M}_{\gamma_{1}, \gamma_{2}} \rightarrow \mathrm{M}_{\gamma_{1}} \times \mathrm{M}_{\gamma_{2}}$. The isomorphism follows from the fact that by the assumptions from Section 7.1 the space $\mathrm{M}_{\gamma_{1}, \gamma_{2}}^{\mathrm{sp}}$ is equal to the intersection $\operatorname{Crit}\left(W_{\gamma}\right) \cap$ $\widetilde{\mathrm{M}}_{\gamma_{1}, \gamma_{2}}^{\mathrm{sp}}$. Hence the sheaf of vanishing cycles of $W_{\gamma}$ vanishes on $\widetilde{\mathrm{M}}_{\gamma_{1}, \gamma_{2}}^{\mathrm{sp}}-$ $\mathrm{M}_{\gamma_{1}, \gamma_{2}}^{\mathrm{sp}}$. 
- $\mathbf{H}_{\mathrm{c}, \mathrm{G}_{\gamma_{1}, \gamma_{2}}}^{\bullet, \text { crit }}\left(\widetilde{\mathrm{M}}_{\gamma_{1}, \gamma_{2}}^{\mathrm{sp}}, W_{\gamma}\right) \rightarrow \mathbf{H}_{\mathrm{c}, \mathrm{G}_{\gamma_{1}, \gamma_{2}}}^{\bullet, \text {,rit }}\left(\widetilde{\mathrm{M}}_{\gamma_{1}, \gamma_{2}}^{\mathrm{sp}}, W_{\gamma_{1}, \gamma_{2}}\right)$, where $W_{\gamma_{1}, \gamma_{2}}$ is the restriction of $W_{\gamma}$ to $\mathrm{M}_{\gamma_{1}, \gamma_{2}}$.

- $\mathbf{H}_{\mathrm{c}, \mathrm{G}_{\gamma_{1}, \gamma_{2}}^{\bullet}}^{\bullet}\left(\widetilde{\mathrm{M}}_{\gamma_{1}, \gamma_{2}}^{\mathrm{sp}}, W_{\gamma_{1}, \gamma_{2}}\right) \simeq \mathbf{H}_{\mathrm{c}, \mathrm{G}_{\gamma_{1}} \times \mathrm{G}_{\gamma_{2}}}^{\bullet, \text { crit }}\left(\mathrm{M}_{\gamma_{1}}^{\mathrm{sp}} \times \mathrm{M}_{\gamma_{2}}^{\mathrm{sp}}, W_{\gamma_{1}} \boxplus W_{\gamma_{2}}\right) \otimes \mathbb{T}^{\mathrm{c}}$, where the isomorphism comes from homotopy equivalence $G_{\gamma_{1}} \times G_{\gamma_{2}} \sim$ $\mathrm{G}_{\gamma_{1}, \gamma_{2}}$, the fact that $\widetilde{\mathrm{M}}_{\gamma_{1}, \gamma_{2}}^{\mathrm{sp}}$ is a bundle over $\mathrm{M}_{\gamma_{1}}^{\mathrm{sp}} \times \mathrm{M}_{\gamma_{2}}^{\mathrm{sp}}$ with affine fibers, and the fact that $W_{\gamma_{1}, \gamma_{2}}$ is the pullback of $W_{\gamma_{1}} \boxplus W_{\gamma_{2}}$. The shift is given by

$$
c=\operatorname{dim} \mathrm{M}_{\gamma_{1}, \gamma_{2}} / \mathrm{G}_{\gamma_{1}, \gamma_{2}}-\operatorname{dim} \mathrm{M}_{\gamma_{1}} / \mathrm{G}_{\gamma_{1}}-\operatorname{dim} \mathrm{M}_{\gamma_{2}} / \mathrm{G}_{\gamma_{2}}=-\chi_{R}\left(\gamma_{2}, \gamma_{1}\right)
$$

- Thom-Sebastiani isomorphism.

The proof of associativity is similar to the one from Section 2.3.

\subsection{Stability conditions and factorization of critical motivic DT-series}

We will use the notation for the Harder-Narasimhan filtration from Section 5.1. Again, we assume for simplicity that all critical values of $W_{\gamma}$ are equal to zero. Stratification by HN-strata gives a spectral sequence converging to $\mathbf{H}_{c, \mathrm{G}_{\gamma}}^{\bullet, \text { crit }}\left(\mathrm{M}_{\gamma}^{\mathrm{sp}}, W_{\gamma}\right)$ with the first term

$$
\bigoplus_{n \geqslant 0} \bigoplus_{\substack{\gamma_{1}, \ldots, \gamma_{n} \in \mathbb{Z}_{\geqslant 0}^{I}-0 \\ \operatorname{Arg} \gamma_{1}>\cdots>\operatorname{Arg} \gamma_{n}}} \mathbf{H}_{\mathrm{c}, \mathrm{G}_{\gamma}}^{\bullet, \text { crit }}\left(\mathrm{M}_{\gamma, \gamma_{\bullet}}^{\mathrm{sp}, \mathrm{HN}}, W_{\gamma}\right)
$$

where $\gamma_{\bullet}=\left(\gamma_{1}, \ldots, \gamma_{n}\right)$ and $\mathrm{M}_{\gamma, \gamma_{\bullet}}^{\mathrm{sp}, \mathrm{HN}}:=\mathrm{M}_{\gamma, \gamma_{\bullet}}^{\mathrm{sp}} \cap \mathrm{M}_{\gamma, \gamma_{\bullet}}^{\mathrm{HN}}$. Then we have

$$
\begin{gathered}
\mathbf{H}_{\mathrm{c}, \mathrm{G}_{\gamma}}^{\bullet, \text { crit }}\left(\mathrm{M}_{\gamma, \gamma_{\bullet}}^{\mathrm{sp}, \mathrm{HN}}, W_{\gamma}\right) \simeq \mathbf{H}_{\mathrm{c}, \mathrm{G}_{\gamma_{1}, \ldots, \gamma_{n}}}^{\bullet, \text { crit }}\left(\mathrm{M}_{\gamma_{1}, \ldots, \gamma_{n}}^{\mathrm{sp}, s s}, W_{\gamma}\right), \\
\mathrm{M}_{\gamma_{1}, \ldots, \gamma_{n}}^{\mathrm{sp}, s s}:=\mathrm{M}_{\gamma}^{\mathrm{sp}} \cap \mathrm{M}_{\gamma_{1}, \ldots, \gamma_{n}}^{s s} .
\end{gathered}
$$

Denote by $\widetilde{M}_{\gamma_{1}, \ldots, \gamma_{n}}^{\mathrm{sp}, s s}$ the pullback of $\mathrm{M}_{\gamma_{1}}^{\mathrm{sp}, s s} \times \cdots \times \mathrm{M}_{\gamma_{n}}^{\mathrm{sp}, s s}$ under the projection $\mathrm{M}_{\gamma_{1}, \ldots, \gamma_{n}} \rightarrow \mathrm{M}_{\gamma_{1}} \times \cdots \times \mathrm{M}_{\gamma_{n}}$. We have an isomorphism

$$
\mathbf{H}_{\mathrm{c}, \mathrm{G}_{\gamma_{1}, \ldots, \gamma_{n}}}^{\bullet, c r i t}\left(\mathrm{M}_{\gamma_{1}, \ldots, \gamma_{n}}^{\mathrm{sp}, s s}, W_{\gamma}\right) \simeq \mathbf{H}_{\mathrm{c}, \mathrm{G}_{\gamma_{1}, \ldots, \gamma_{n}}}^{\bullet, c r i t}\left(\widetilde{\mathrm{M}}_{\gamma_{1}, \ldots, \gamma_{n}}^{\mathrm{sp}, s s}, W_{\gamma}\right)
$$

because $W_{\gamma}$ has no critical points on $\widetilde{\mathrm{M}}_{\gamma_{1}, \ldots, \gamma_{n}}^{\mathrm{sp}, s s}-\mathrm{M}_{\gamma_{1}, \ldots, \gamma_{n}}^{\mathrm{sp}, s s}$. The next step is an isomorphism

$$
\mathbf{H}_{\mathrm{c}, \mathrm{G}_{\gamma_{1}, \ldots, \gamma_{n}}}^{\bullet, \text { crit }}\left(\widetilde{\mathrm{M}}_{\gamma_{1}, \ldots, \gamma_{n}}^{\mathrm{sp}, \text { ss }}, W_{\gamma}\right) \simeq \mathbf{H}_{\mathrm{c}, \mathrm{G}_{\gamma_{1}, \ldots, \gamma_{n}}}^{\bullet, \mathrm{crit}}\left(\widetilde{\mathrm{M}}_{\gamma_{1}, \ldots, \gamma_{n}}^{\mathrm{sp}, s s},\left(W_{\gamma}\right)_{\mid \mathrm{M}_{\gamma_{1}, \ldots, \gamma_{n}}}\right) .
$$


This is the key point. This isomorphism follows iteratively from the integral identity proven in the next section; see Corollary 7.1. Finally, using the fact that $\widetilde{\mathrm{M}}_{\gamma_{1}, \ldots, \gamma_{n}}^{\mathrm{sp}, s s}$ is an affine bundle over $\mathrm{M}_{\gamma_{1}}^{\mathrm{sp}, s s} \times \cdots \times \mathrm{M}_{\gamma_{n}}^{\mathrm{sp}, s s}$ and that the function $\left(W_{\gamma}\right)_{\mid \mathrm{M}_{\gamma_{1}, \ldots, \gamma_{n}}}$ is the pullback of $W_{\gamma_{1}} \boxplus \cdots \boxplus W_{\gamma_{n}}$, we obtain that

$$
\begin{gathered}
\mathbf{H}_{\mathrm{c}, \mathrm{G}_{\gamma}}^{\bullet, \text { crit }}\left(\mathrm{M}_{\gamma, \gamma_{\bullet}}^{\mathrm{sp}, \mathrm{HN}}, W_{\gamma}\right) \simeq \mathbf{H}_{\mathrm{c}, \mathrm{G}_{\gamma_{1}} \times \cdots \times \mathrm{G}_{\gamma_{n}}}^{\bullet, \text { crit }}\left(\mathrm{M}_{\gamma_{1}}^{\mathrm{sp}, s s} \times \cdots \times \mathrm{M}_{\gamma_{n}}^{\mathrm{sp}, s s}, W_{\gamma_{1}} \boxplus \cdots \boxplus W_{\gamma_{n}}\right) \\
\otimes \mathbb{T}^{\otimes\left(-\sum_{i<j} \chi_{R}\left(\gamma_{j}, \gamma_{i}\right)\right)}
\end{gathered}
$$

Applying Thom-Sebastiani isomorphism and duality we obtain the Factorization Formula for the critical motivic DT-series, as in Section 5.3.

\subsection{Integral identity}

Let $X$ be an algebraic variety over $\mathbb{C}$, and $\mathcal{E}_{1}, \mathcal{E}_{2}$ be two vector bundles over $X$. Denote by $Y$ the total space of the bundle $\mathcal{E}_{1} \oplus \mathcal{E}_{2}$, and by $Y^{(1)} \subset Y$ the total space of $\mathcal{E}_{1}$. Assume that we are given a function

$$
f \in \mathcal{O}(Y)^{\mathbb{G}_{m}}
$$

where the group $\mathbb{G}_{m}$ acts linearly along fibers of $\mathcal{E}_{1} \oplus \mathcal{E}_{2}$ with weights +1 on $\mathcal{E}_{1}$ and -1 on $\mathcal{E}_{2}$.

Theorem 7.1. Let $Y_{0}=f^{-1}(0) \subset Y$ be the zero locus of $f$ and $Y_{0}^{(1)}:=$ $Y_{0} \cap Y^{(1)}$. Then

$$
\left.\left(Y_{0}^{(1)} \rightarrow X\right) !\left(Y_{0}^{(1)} \rightarrow Y_{0}\right)^{*} \operatorname{Cone}\left(\phi_{f} \mathbb{Z}_{Y} \rightarrow \phi_{f}\left(Y^{(1)} \rightarrow Y\right)_{*} \mathbb{Z}_{Y^{(1)}}\right)\right)=0
$$

Corollary 7.1. For any closed subset $X^{\mathrm{sp}} \subset X$ such that its preimage $Y^{\mathrm{sp}}$ under the projection $Y^{(1)} \rightarrow X$ lies in $Y_{0}$, we have a canonical isomorphism

$$
\mathbf{H}_{\mathrm{c}}^{\bullet, c r i t}\left(Y^{\mathrm{sp}}, f\right) \simeq \mathbf{H}_{\mathrm{c}}^{\bullet, \text { crit }}\left(Y^{\mathrm{sp}}, f_{\mid Y^{(1)}}\right) \text {. }
$$

Moreover, the same conclusion holds in the equivariant setting.

Proof of the corollary. There is an obvious morphism in $D^{b}$ (MMHS)

$$
\alpha: H_{\mathrm{c}, \mathrm{MMHS}}^{\bullet}\left(Y^{\mathrm{sp}}, f\right) \rightarrow H_{\mathrm{c}, \mathrm{MMHS}}^{\bullet, \mathrm{crit}}\left(Y^{\mathrm{sp}}, f_{\mid Y^{(1)}}\right) .
$$

We claim that $\phi$ is an isomorphism, i.e., the cone of $\alpha$ is zero. By faithfulness, it is sufficient to prove the vanishing using only Betti realization $\alpha_{\text {Betti. }}$. 
Denote by $\mathcal{E}$ the complex of constructible sheaves whose vanishing is claimed in Theorem 7.1. Then we have

$$
\left(X^{\mathrm{sp}} \rightarrow p t\right)_{!}\left(X^{\mathrm{sp}} \rightarrow X\right)^{*} \mathcal{E} \otimes \mathbb{Q} \simeq \operatorname{Cone}\left(\alpha_{\text {Betti }}\right)
$$

Hence, we proved the result in the non-equivariant case. The extension to the equivariant setting is straightforward.

Proof of Theorem 7.1. Let us consider an affine morphism $Z \rightarrow X$ whose fiber $Z_{x}$ at any point $x \in X$ is $\operatorname{Spec} \mathcal{O}\left((Y \rightarrow X)^{-1}(x)\right)^{\mathbb{G}_{m}}$. There is an obvious map $Y \rightarrow Z$, and the function $f$ is the pullback of a function $f_{Z} \in \mathcal{O}(Z)$. We will construct a completion $\bar{Y} \supset Y$ such that the projection $Y \rightarrow Z$ extends to a proper map $\pi: \bar{Y} \rightarrow Z$. In what follows, we will compose $\pi$ with the projection $Z \rightarrow X$ and describe $\bar{Y}$ as a fibration over $X$. Namely, for any $x \in X$ consider the product of two projective spaces

$$
\mathbb{P}\left(\mathcal{E}_{1, x} \oplus \mathbb{C}\right) \times \mathbb{P}\left(\mathcal{E}_{2, x} \oplus \mathbb{C}\right) \simeq\left(\mathcal{E}_{1, x} \cup \mathbb{P} \mathcal{E}_{1, x}\right) \times\left(\mathcal{E}_{2, x} \cup \mathbb{P} \mathcal{E}_{2, x}\right),
$$

make blow-up at two disjoint submanifolds $\mathbb{P} \mathcal{E}_{1, x} \times\left\{0_{\mathcal{E}_{2, x}}\right\}$ and $\left\{0_{\mathcal{E}_{1, x}}\right\} \times$ $\mathbb{P} \mathcal{E}_{2, x}$ (here $0_{\mathcal{E}_{1, x}}, 0_{\mathcal{E}_{2, x}}$ are zero points in fibers $\mathcal{E}_{1, x}$ and $\mathcal{E}_{2, x}$, respectively), and remove the proper transform of divisors $\mathbb{P} \mathcal{E}_{1, x} \times \mathcal{E}_{2, x}$ and $\mathcal{E}_{1, x} \times \mathbb{P} \mathcal{E}_{2, x}$. The result is by definition the fiber of $\bar{Y}$ over $x$. This fiber is smooth, and carries a natural stratification with eight smooth strata. The incidence diagram of strata is

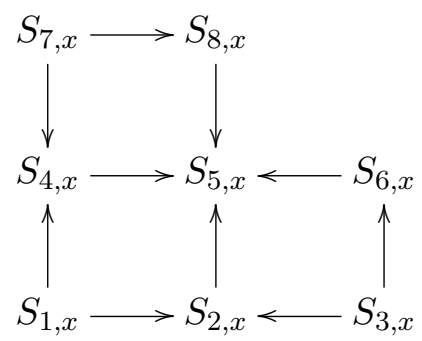

where all arrows are inclusions. Open parts of the strata are

$$
\begin{array}{ccc}
\mathbb{P} \mathcal{E}_{2, x} & S_{8, x} \\
\mathcal{E}_{2, x}^{\neq 0} & \mathcal{E}_{1, x}^{\neq 0} \times \mathcal{E}_{2, x}^{\neq 0} & S_{6, x} \\
\left\{0_{\left.\mathcal{E}_{1, x} \oplus \mathcal{E}_{2, x}\right\}}\right. & \mathcal{E}_{1, x}^{\neq 0} & \mathbb{P} \mathcal{E}_{1, x}
\end{array}
$$


where $\mathcal{E}_{i, x}^{\neq 0}:=\mathcal{E}_{i, x}-\left\{0_{\mathcal{E}_{i, x}}\right\}, i=1,2$ and $S_{6, x} \simeq S_{8, x} \simeq\left(\mathcal{E}_{1, x}^{\neq 0} \times \mathcal{E}_{2, x}^{\neq 0}\right) / \mathbb{G}_{m, \mathbb{C}}$. Under the projection to the fiber $Z_{x}$ of $Z \rightarrow X$ the strata $S_{1, x}, S_{2, x}, S_{3, x}, S_{4, x}$, $S_{7, x}$ are mapped to the base point in $Z_{x}$, whereas the union $S_{5, x} \cup S_{6, x} \cup S_{8, x}$ is a fibration with fiber $\mathbb{P}^{1}$ over an open stratum in $Z_{x}$ isomorphic to $S_{6, x}$. Varying point $x \in X$ we obtain strata $\left(S_{i}\right)_{1 \leqslant i \leqslant 8}$ of $\bar{Y}$ with open parts $\left(S_{i}^{\circ}\right)_{1 \leqslant i \leqslant 8}$. The open subset $Y \subset \bar{Y}$ is a union of strata, $Y=S_{1}^{\circ} \sqcup S_{2}^{\circ} \sqcup$ $S_{4}^{\circ} \sqcup S_{5}^{\circ}$.

Now return to the proof of identity. By base change it is sufficient to prove that for any $x \in X$ such that $f_{X}(x)=0$ (here $f_{X}$ is the pullback of $f$ via the embedding $X \hookrightarrow Y$ as the zero section) we have

$\left(\mathcal{E}_{1, x} \rightarrow p t\right)_{!}\left(\mathcal{E}_{1, x} \rightarrow Y_{0}\right)^{*} \phi_{f} \mathcal{G}^{\bullet}=0, \quad \mathcal{G}^{\bullet}:=\operatorname{Cone}\left(\mathbb{Z}_{Y} \rightarrow\left(Y^{(1)} \rightarrow Y\right)_{*} \mathbb{Z}_{Y^{(1)}}\right)$.

We will construct certain extension $\mathcal{F}^{\bullet}$ of $\mathcal{G}^{\bullet}$ from $Y$ to $\bar{Y}$. Let $\mathcal{F}_{1}$ be the extension of $\mathbb{Z}_{Y}$ by ! to $S_{3}^{\circ} \sqcup S_{6}^{\circ}$, and by $*$ to $S_{7}^{\circ} \sqcup S_{8}^{\circ}$. Similarly we define $\mathcal{F}_{2}$ to be the extension of $\mathbb{Z}_{Y^{(1)}}$ by ! to $\bar{Y}$. Restrictions of $\mathcal{F}_{1}$ and $\mathcal{F}_{2}$ to $Y \subset \bar{Y}$ coincide with the sheaves $\mathbb{Z}_{Y}$ and $\left(Y^{(1)} \rightarrow Y\right)_{*} \mathbb{Z}_{Y^{(1)}}$, respectively. There is a natural morphism $\mathcal{F}_{1} \rightarrow \mathcal{F}_{2}$, and the restriction of $\mathcal{F}^{\bullet}:=\operatorname{Cone}\left(\mathcal{F}_{1} \rightarrow \mathcal{F}_{2}\right)$ to $Y$ is isomorphic to $\mathcal{G}^{\bullet}$.

Lemma 7.1. The complexes

$$
\left(\mathcal{E}_{1, x} \rightarrow p t\right)_{!}\left(\mathcal{E}_{1, x} \rightarrow Y_{0}\right)^{*} \phi_{f} \mathcal{G}^{\bullet} \simeq\left(\mathcal{E}_{1, x} \rightarrow p t\right)_{!}\left(\mathcal{E}_{1, x} \rightarrow \bar{Y}_{0}\right)^{*} \phi_{f_{\bar{Y}}} \mathcal{F}^{\bullet}
$$

and

$$
\left(\bar{Y}_{x}^{\prime} \rightarrow p t\right)_{!}\left(\bar{Y}_{x}^{\prime} \rightarrow \bar{Y}_{0}\right)^{*} \phi_{f_{\bar{Y}}} \mathcal{F}^{\bullet}
$$

are isomorphic. Here $\bar{Y}_{x}^{\prime}:=S_{1, x}^{\circ} \sqcup S_{2, x}^{\circ} \sqcup S_{3, x}^{\circ} \sqcup S_{4, x}^{\circ} \sqcup S_{7, x}^{\circ}$ is a closed subset of the fiber of $\bar{Y}$ over $x$, function $f_{\bar{Y}}=(\bar{Y} \rightarrow Z)^{*} f_{Z}$ is the extension of $f$ by continuity, and $\bar{Y}_{0}$ is zero locus of $f_{\bar{Y}}$.

Proof of Lemma. Notice that $\bar{Y}_{x}^{\prime}=\mathcal{E}_{1, x} \sqcup S_{3, x}^{\circ} \sqcup S_{4, x}^{\circ} \sqcup S_{7, x}^{\circ}$. By spectral sequences it is sufficient to check that

1) $\left(S_{3, x}^{\circ} \rightarrow p t\right) !\left(S_{3, x}^{\circ} \rightarrow \bar{Y}_{0}\right)^{*} \phi_{f_{\bar{Y}}} \mathcal{F}^{\bullet}=0$,

2) $\left(\left(S_{4, x}^{\circ} \sqcup S_{7, x}^{\circ}\right) \rightarrow p t\right) !\left(\left(S_{4, x}^{\circ} \sqcup S_{7, x}^{\circ}\right) \rightarrow \bar{Y}_{0}\right)^{*} \phi_{f_{\bar{Y}}} \mathcal{F}^{\bullet}=0$.

First vanishing follows from the observation that the closed subspace $V:=S_{3}^{\circ} \sqcup S_{6}^{\circ}$ of $\bar{Y}$ has an open neighborhood $U:=S_{2}^{\circ} \sqcup S_{3}^{\circ} \sqcup S_{5}^{\circ} \sqcup S_{6}^{\circ}$ which is the total space of a line bundle over $S_{3}^{\circ} \sqcup S_{6}^{\circ}$. Restriction of function $f_{\bar{Y}}$ to $U$ is the pullback of a function on $V$, and the restriction of the sheaf $\mathcal{F}^{\bullet}$ 
to $U$ is the extension by zero of the constant sheaf on $U-V$. Hence, the functor of vanishing cycles can be interchanged with the restriction to $V$, and therefore

$$
\left(\left(\left(S_{3}^{\circ} \sqcup S_{6}^{\circ}\right) \cap \bar{Y}_{0}\right) \rightarrow \bar{Y}_{0}\right)^{*} \phi_{f_{\bar{Y}}} \mathcal{F}^{\bullet}=0
$$

This implies 1).

Similarly, $V^{\prime}:=S_{7}^{\circ} \sqcup S_{8}^{\circ}$ is a base of a line bundle with the total space $U^{\prime}=S_{4}^{\circ} \sqcup S_{5}^{\circ} \sqcup S_{7}^{\circ} \sqcup S_{8}^{\circ}$. Restriction of function $f_{\bar{Y}}$ to $U^{\prime}$ is the pullback of a function on $V^{\prime}$, and the restriction of the sheaf $\mathcal{F}^{\bullet}$ to $U$ is the direct image of the constant sheaf on $U^{\prime}-V^{\prime}$. Hence, we have

$$
\left(U_{0}^{\prime} \rightarrow V_{0}^{\prime}\right) !\left(U_{0}^{\prime} \rightarrow \bar{Y}_{0}\right)^{*} \mathcal{F}^{\bullet}=0, \quad U_{0}^{\prime}:=U \cap \bar{Y}_{0}, V_{0}^{\prime}:=V \cap \bar{Y}_{0}
$$

The reason is that $(\mathbb{C} \rightarrow p t) !\left(\mathbb{C}^{*} \rightarrow \mathbb{C}\right)_{*} \mathbb{Z}_{\mathbb{C}^{*}}=0$. This proves the second vanishing.

Using the fact that the functor of vanishing cycles commutes with proper morphisms (e.g., with $\pi: \bar{Y} \rightarrow Z$ ), we obtain an isomorphism

$$
\left(\bar{Y}_{x}^{\prime} \rightarrow p t\right) !\left(\bar{Y}_{x}^{\prime} \rightarrow \bar{Y}_{0}\right)^{*} \phi_{f_{\bar{Y}}} \mathcal{F}^{\bullet} \simeq\left(p t \rightarrow f_{Z}^{-1}(0)\right)^{*} \phi_{Z}(\bar{Y} \rightarrow Z)_{*} \mathcal{F}^{\bullet}
$$

where the inclusion of the point to $f_{Z}^{-1}(0)$ is given by the base point at the fiber $Z_{x} \subset f_{Z}^{-1}(0)$. Now we apply the following lemma.

Lemma 7.2. The direct image $(\bar{Y} \rightarrow Z)_{*} \mathcal{F}^{\bullet}$ vanishes.

Proof of Lemma. The morphism $\pi: \bar{Y} \rightarrow Z$ is proper, hence we can use the base change, and it is sufficient to prove that for any point $z \in Z$ the direct image

$$
\left(\pi^{-1}(z) \rightarrow p t\right)_{*}\left(\pi^{-1}(z) \rightarrow \bar{Y}\right)^{*} \mathcal{F}^{\bullet}=0 .
$$

Denote by $i: X \hookrightarrow Z$ the canonical embedding given by base points of cones $\left(Z_{x}\right)_{x \in X}$.

For $z \in Z-i(X)$ the fiber $\pi^{-1}(z)$ is the projective line $\mathbb{P}_{\mathbb{C}}^{1}$. The restriction of the sheaf $\mathcal{F}^{\bullet}$ to $\pi^{-1}(z)$ is a constant sheaf on $\mathbb{A}_{\mathbb{C}}^{1}$ extended by zero to the point $\infty \in \mathbb{P}_{\mathbb{C}}^{1}$. Hence $R \Gamma$ of this sheaf on $\pi^{-1}(z)$ vanishes.

For a point $z \in i(X)$, the fiber $\pi^{-1}(z)$ is the joint of two projective spaces identified at zero:

$$
\pi^{-1}(z)=\mathbb{P}\left(\mathcal{E}_{1, x} \oplus \mathbb{C}\right) \cup_{0} \mathbb{P}\left(\mathcal{E}_{2, x} \oplus \mathbb{C}\right)=S_{1, x}^{\circ} \sqcup S_{2, x}^{\circ} \sqcup S_{3, x}^{\circ} \sqcup S_{4, x}^{\circ} \sqcup S_{7, x}^{\circ},
$$

where the point $x \in X$ corresponds to $z$. The restriction of $\mathcal{F}^{\bullet}$ to $\pi^{-1}(z)$ is the constant sheaf on the open part $S_{1, x}^{\circ} \sqcup S_{2, x}^{\circ} \sqcup S_{4, x}^{\circ}$ extended by zero to 
$S_{3, x}^{\circ}$, and by the direct image to $S_{7, x}^{\circ}$. Then a direct calculation shows that $R \Gamma$ of $\mathcal{F}_{\mid \pi^{-1}(z)}^{\bullet}$ vanishes. This proves the lemma.

Two above lemmas imply that for any point $x \in f_{X}{ }^{-1}(0)$ we have

$$
\left(\mathcal{E}_{1, x} \rightarrow p t\right)_{!}\left(\mathcal{E}_{1, x} \rightarrow Y_{0}\right)^{*} \phi_{f} \mathcal{G}^{\bullet}=0 .
$$

By base change this implies the Theorem.

\subsection{Factorization systems in the critical case}

The proof of the integrality property is based on the theory of factorization systems from Sections 6.5 and $6.6^{15}$.

First, for $\gamma \in \mathbb{Z}_{\geqslant 0}^{I}$ we define the following graded space:

$$
\mathcal{H}_{\gamma}^{\prime}:=D\left(\mathbf{H}_{c, \mathbf{T}_{\gamma}}^{\bullet, \text { crit }}\left(\mathrm{M}_{\gamma}^{\mathrm{sp}}, W_{\gamma}\right)\right) \otimes \mathbb{T}^{\otimes \operatorname{dim} \mathrm{M}_{\gamma} / \mathbf{T}_{\gamma}} .
$$

Our goal is to define a structure of a non-singular factorization system on the collection $\left(\mathcal{H}_{\gamma}^{\prime}\right)_{\gamma \in \mathbb{Z}_{\geqslant 0}^{I}}$. Then applying Theorem 6.4 and Corollary 6.3 from Section 6.6, we will deduce admissibility of the motivic Donaldson-Thomas series in the critical case.

First, we claim that $\mathcal{H}_{\gamma}^{\prime}$ is a finitely generated module over $\mathbf{H}^{\bullet}\left(\mathrm{BT}_{\gamma}\right)$. This is a particular case of a more general result proven below, where we identify cohomology with coefficients in the sheaf of vanishing cycles with cohomology of certain real semi-algebraic sets.

We define the multiplication map (for $\gamma=\gamma_{1}+\gamma_{2}$ ) as the dual to the composition

$$
\begin{aligned}
& \underset{\mathrm{c}, \mathbf{T}_{\gamma}}{\mathbf{\bullet}}\left(\mathrm{M}_{\gamma}^{\mathrm{sp}}, W_{\gamma}\right) \rightarrow \mathbf{H}_{\mathrm{c}, \mathrm{T}_{\gamma}}^{\bullet, \text { crit }}\left(\mathrm{M}_{\gamma_{1}}^{\mathrm{sp}} \times \mathrm{M}_{\gamma_{2}}^{\mathrm{sp}}, W_{\gamma}\right) \rightarrow \mathbf{H}_{\mathrm{c}, \mathbf{T}_{\gamma}}^{\bullet, \text { crit }}\left(\mathrm{M}_{\gamma_{1}}^{s}\right. \\
& \left.\quad \times \mathrm{M}_{\gamma_{2}}^{\mathrm{sp}}, W_{\gamma_{1}} \boxplus W_{\gamma_{2}}\right),
\end{aligned}
$$

where the first arrow is the restriction to the closed subspace $\mathrm{M}_{\gamma_{1}}^{\mathrm{sp}} \times \mathrm{M}_{\gamma_{2}}^{\mathrm{sp}} \subset$ $\mathrm{M}_{\gamma}^{\mathrm{sp}}$, and the second arrow comes from the canonical morphism of sheaves. It is clear that the constraints of commutativity, associativity and of the equivariance of the product are satisfied.

Theorem 7.2. In the notation from Section 6.4 the multiplication map induces an isomorphism of coherent sheaves on the open sets $U_{\gamma_{1}, \gamma_{2}}$.

\footnotetext{
${ }^{15}$ We do not know how to make a shortcut similar to the one in Section 6.7.
} 
Proof ${ }^{16}$. We will use Betti realization of the critical cohomology cohomology (with coefficients in $\mathbb{Q}$ ). We have to prove that the dual $\mathbb{Z}$-graded vector space over $\mathbb{Q}$ to

$$
H_{\mathrm{c}, \mathrm{T}_{\gamma}}^{\bullet}\left(\mathrm{M}_{\gamma}^{\mathrm{sp}},\left(\mathrm{M}_{\gamma}^{\mathrm{sp}} \hookrightarrow W_{\gamma}^{-1}(0)\right)^{*} \phi_{W_{\gamma}}\left(\operatorname{Cone}\left(\mathbb{Q}_{\mathrm{M}_{\gamma}} \rightarrow i_{*} \mathbb{Q}_{\mathrm{M}_{\gamma_{1}} \times \mathrm{M}_{\gamma_{2}}}\right)\right)\right)
$$

where $i: \mathrm{M}_{\gamma_{1}} \times \mathrm{M}_{\gamma_{2}} \rightarrow \mathrm{M}_{\gamma}$ is the obvious embedding, is a finitely generated $H^{\bullet}\left(\mathrm{BT}_{\gamma}, \mathbb{Q}\right)$-module with support on $\operatorname{Spec} H^{\bullet}\left(\mathrm{BT}_{\gamma}, \mathbb{Q}\right)-U_{\gamma_{1}, \gamma_{2}}$.

First, notice that in the above formula we can replace complex torus $\mathrm{T}_{\gamma}$ by its maximal compact real subtorus $\mathrm{T}_{\gamma}^{\mathrm{c}}$ (hence the shift is replaced by the real dimension of the contractible group $\left.\mathrm{T}_{\gamma} / \mathrm{T}_{\gamma}^{\mathrm{c}}\right)$.

Also notice that we can replace the sheaf of vanishing cycles by the sheaf of nearby cycles. Indeed, these two choices (i.e., $\phi_{W_{\gamma}}$ and $\psi_{W_{\gamma}}$ ) are related by the exact triangle from Section 7.2 with the cohomology of the third vertex of the triangle given by

$$
\begin{aligned}
& H_{\mathrm{c}, \mathrm{T}_{\gamma}}^{\bullet}\left(\mathrm{M}_{\gamma}^{\mathrm{sp}},\left(\mathrm{M}_{\gamma}^{\mathrm{sp}} \rightarrow \mathrm{M}_{\gamma}\right)^{*}\left(\operatorname { C o n e } \left(\mathbb{Q}_{\mathrm{M}_{\gamma}} \rightarrow i_{*} \mathbb{Q}_{\left.\left.\left.\mathrm{M}_{\gamma_{1}} \times \mathrm{M}_{\gamma_{2}}\right)\right)\right)}\right.\right.\right. \\
& \quad \simeq H_{\mathrm{c}, \mathrm{T}_{\gamma}}^{\bullet}\left(\mathrm{M}_{\gamma}^{\mathrm{sp}}-\left(\mathrm{M}_{\gamma_{1}}^{\mathrm{sp}} \times \mathrm{M}_{\gamma_{2}}^{\mathrm{sp}}\right)\right) .
\end{aligned}
$$

Then we can use Proposition 6.3 from Section 6.5.

Now we have reduced the question to the one about equivariant cohomology with coefficients in the sheaves of nearby cycles. Let us consider the following general situation. Suppose we are given a complex smooth algebraic variety $X$ endowed with an algebraic action of a complex torus $\mathrm{T}$ as well as a T-invariant function $f \in \mathcal{O}(X)$, and a closed T-invariant algebraic set $S \subset f^{-1}(0)$. We want to calculate

$$
H_{\mathrm{c}, \mathrm{T}}\left(S,\left(S \rightarrow f^{-1}(0)\right)^{*} \psi_{f} \mathbb{Q}_{X}\right)
$$

As above, we can replace $\mathrm{T}$ by the maximal compact real subtorus $\mathrm{T}^{\mathrm{c}} \subset \mathrm{T}$. Let us choose $\mathrm{T}^{\mathrm{c}}$-invariant continuous real semi-algebraic maps $r, s: X \rightarrow$ $[0,+\infty)$ such that:

(1) the map $r$ is proper,

(2) $d^{-1}(0)=S$ (the map $d$ can be thought of as a "distance to $S$ ").

\footnotetext{
${ }^{16}$ We thank to Joerg Schürmann for a discussion that led to the proof below.
} 
Then we claim that $H_{\mathrm{c}, \mathrm{T}^{\mathrm{c}}}\left(S,\left(S \rightarrow f^{-1}(0)\right)^{*} \psi_{f} \mathbb{Q}_{X}\right)$ is isomorphic to the iterated $\operatorname{limit}^{17}$

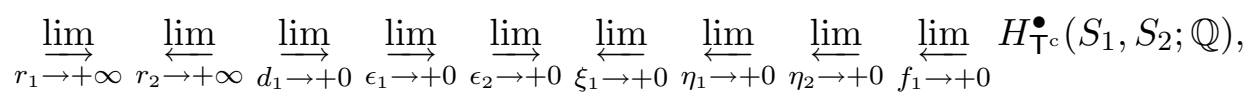

where $\left(S_{1}, S_{2}\right)$ is the following pair of $\mathrm{T}^{\mathrm{c}}$-invariant compact real semialgebraic sets in $X$ depending on positive real parameters $r_{1}, r_{2}, d_{1}, \epsilon_{1}, \epsilon_{2}$, $\xi_{1}, \eta_{1}, \eta_{2}, f_{1}$ :

$$
\begin{aligned}
& S_{1}=\left\{x \in X \mid r(x) \leqslant r_{2}+\epsilon_{2}-\eta_{2}, f(x) \geqslant f_{1}, d(x) \leqslant d_{1}-\xi_{1}\right\}, \\
& S_{2}=\left\{x \in X \mid r_{1}-\epsilon_{1}+\eta_{1} \leqslant r(x) \leqslant r_{2}+\epsilon_{2}-\eta_{2}, f(x) \geqslant f_{1}, d(x) \leqslant d_{1}-\xi_{1}\right\} .
\end{aligned}
$$

This description of cohomology is similar to "semi-global" Milnor fibrations from [51].

Let us explain the above sequence of direct and inverse limits. First pair of limits ${\stackrel{\lim }{\longrightarrow} r_{1} \rightarrow+\infty}_{\lim _{r_{2} \rightarrow+\infty}}$ corresponds to the fact that the cohomology of $S$ with compact support with coefficient in any sheaf can be calculated as the double limit of cohomology of pair $\left(K_{1}, K_{2}\right)$ of compact sets depending on parameters $r_{1}, r_{2}$ given by

$$
K_{1}:=\left\{x \in S \mid r(x) \leqslant r_{2}\right\}, \quad K_{2}:=\left\{x \in S \mid r_{1} \leqslant r(x) \leqslant r_{2}\right\}
$$

By definition of $\psi_{f} \mathbb{Q}_{X}$, we should calculate cohomology of pair $\left(K_{1}, K_{2}\right)$ with coefficients in the sheaf $\left(X_{+} \rightarrow X\right)_{*} \mathbb{Q}_{X_{+}}$on $X$, where $X_{+}:=f^{-1}\left(\mathbb{R}_{>0}\right)$. Then we consider a system of open neighborhoods $U_{1}, U_{2}$ of $K_{1}, K_{2}$ in $X$, depending on small positive parameters $d_{1}, \epsilon_{1}, \epsilon_{2}$ and given by

$$
\begin{aligned}
& \left.U_{1}:=\left\{x \in X \mid r(x)<r_{2}+\epsilon_{2}\right), d(x)<d_{1}\right\}, \\
& U_{2}:=\left\{x \in X \mid r_{2}-\epsilon_{1}<r(x)<r_{2}+\epsilon_{2}, d(x)<d_{1}\right\} .
\end{aligned}
$$

This is a fundamental system of neighborhoods (as follows from the properness of the map $\left.(r, d): X \rightarrow \mathbb{R}^{2}\right)$. Hence, the cohomology of the pair $\left(K_{1}, K_{2}\right)$ is the inductive limit of the cohomology of the pair $\left(U_{1}, U_{2}\right)$ as $d_{1}, \epsilon_{1}, \epsilon_{2} \rightarrow$ +0 . Now we should calculate the cohomology $H^{\bullet}\left(U_{1} \cap X_{+}, U_{2} \cap X_{+} ; \mathbb{Q}\right)$. We approximate locally compact spaces $U_{i} \cap X_{+}, i=1,2$ from inside by a growing family of compact subsets $S_{i}, i=1,2$ depending on small positive parameters $\xi_{1}, \eta_{1}, \eta_{2}, f_{1}$. This finishes the explanation of the multiple limit formula

\footnotetext{
${ }^{17}$ More precisely, we should work in the derived category and take derived projective limits. In our case, all cohomology spaces will have bounded finite dimension, hence the derived projective limit coincides with the usual one.
} 
for $H_{c, T^{c}}\left(S,\left(S \rightarrow f^{-1}(0)\right)^{*} \psi_{f} \mathbb{Q}_{X}\right)$. One can simplify slightly this formula, by reducing to the case

$$
d_{1}=\epsilon_{1}=\epsilon_{2}, \quad \xi_{1}=\eta_{1}=\eta_{2}=f_{1}
$$

The above cohomology depends on all parameters in a constructible way in the sense of the standard real semi-algebraic structure on $\mathbb{R}$. This is clear for non-equivariant cohomology. In the case of equivariant cohomology, we should use the stratification given by the dimension of stabilizer in $\mathrm{T}^{\mathrm{c}}$ of a point $x \in X$. Therefore, at every stage the limit stabilizes for sufficiently small values of the parameters $1 / r_{1}, 1 / r_{2}, d_{1}, \epsilon_{1}, \epsilon_{2}, \xi_{1}, \eta_{1}, \eta_{2}, f_{1}$. Hence, the space $H_{\mathrm{c}, \mathrm{T}^{\mathrm{c}}}\left(S,\left(S \rightarrow f^{-1}(0)\right)^{*} \psi_{f} \mathbb{Q}_{X}\right)$ coincides with the cohomology with compact support of the semi-algebraic set $S_{1}-S_{2}$ for certain values of parameters, and therefore the $\mathbb{Z}$-graded dual space is a finitely generated $H^{\bullet}\left(\mathrm{BT}^{\mathrm{c}}, \mathbb{Q}\right)$-module.

Now we can apply the iterated limit formula in two cases: one with

$$
\begin{aligned}
& \mathrm{T}=\mathrm{T}_{\gamma}, X=\mathrm{M}_{\gamma}, f=W_{\gamma}, S=\mathrm{M}_{\gamma}^{\mathrm{sp}} \text { and another one with } \\
& \mathrm{T}^{\prime}=\mathrm{T}=\mathrm{T}_{\gamma}, X^{\prime}=\mathrm{M}_{\gamma_{1}} \times \mathrm{M}_{\gamma_{2}} \subset X, f=W_{\gamma_{1}} \boxplus W_{\gamma_{2}}=f_{\mid X^{\prime}} \\
& S^{\prime}=\mathrm{M}_{\gamma_{1}}^{\mathrm{sp}} \times \mathrm{M}_{\gamma_{2}}^{\mathrm{sp}}=S \cap X^{\prime} .
\end{aligned}
$$

We also choose the functions $r, d$ on $X$ as above, and define $r^{\prime}, d^{\prime}$ to be the restrictions of $r, d$ to $X^{\prime}$. Then we have equalities

$$
S_{1}^{\prime}=S_{1} \cap X^{\prime}, S_{2}^{\prime}=S_{2} \cap X^{\prime}
$$

and a long exact sequence

$$
\cdots \rightarrow H_{\mathrm{\top}}^{\bullet}\left(S_{1}, S_{2} \cup_{S_{2}^{\prime}} S_{1}^{\prime} ; \mathbb{Q}\right) \rightarrow H_{\mathrm{\top}}^{\bullet}\left(S_{1}, S_{2} ; \mathbb{Q}\right) \rightarrow H_{\mathrm{\top}}^{\bullet}\left(S_{1}^{\prime}, S_{2}^{\prime} ; \mathbb{Q}\right) \rightarrow \ldots
$$

For sufficiently small values of parameters as above we can replace $H_{\mathrm{\top}}^{\bullet}\left(S_{1}, S_{2} ; \mathbb{Q}\right)$ and $H_{\mathrm{\top}}^{\bullet}\left(S_{1}^{\prime}, S_{2}^{\prime}\right)$ by

$$
\begin{aligned}
& H_{\mathrm{c}, \mathrm{T}_{\gamma}}^{\bullet}\left(\mathrm{M}_{\gamma}^{\mathrm{sp}},\left(\mathrm{M}_{\gamma}^{\mathrm{sp}} \hookrightarrow W_{\gamma}^{-1}(0)\right)^{*} \phi_{W_{\gamma}} \mathbb{Q}_{\mathrm{M}_{\gamma}}\right) \text { and } \\
& H_{\mathrm{c}, \mathrm{T}_{\gamma}}^{\bullet}\left(\mathrm{M}_{\gamma_{1}}^{\mathrm{sp}} \times \mathrm{M}_{\gamma_{2}}^{\mathrm{sp}},\left(\mathrm{M}_{\gamma_{1}}^{\mathrm{sp}} \times \mathrm{M}_{\gamma_{2}}^{\mathrm{sp}} \hookrightarrow\left(W_{\gamma_{1}} \boxplus W_{\gamma_{2}}\right)^{-1}(0)\right)^{*} \phi_{W_{\gamma_{1}}} \boxplus W_{\gamma_{2}} \mathbb{Q}_{\mathrm{M}_{\gamma_{1}} \times \mathrm{M}_{\gamma_{2}}}\right),
\end{aligned}
$$

respectively. 
The equivariant cohomology $H_{\top}^{\bullet}\left(S_{1}, S_{2} \cup_{S_{2}^{\prime}} S_{1}^{\prime} ; \mathbb{Q}\right)$ coincides with the equivariant cohomology with compact support

$$
H_{c, \mathrm{~T}}^{\bullet}\left(S_{1}-\left(S_{2} \cup_{S_{2}^{\prime}} S_{1}^{\prime}\right), \mathbb{Q}\right)
$$

of a locally closed real semi-algebraic set, which is contained in $X-X^{\prime}=$ $\mathrm{M}_{\gamma}-\left(\mathrm{M}_{\gamma_{1}} \times \mathrm{M}_{\gamma_{2}}\right)$. Applying Proposition 6.3, we finish the proof of theorem.

\subsection{Comparison with motivic DT-invariants from [34]}

Results of Sections 7.1-7.9 ensure the existence of the critical COHA $\mathcal{H}^{\text {crit }}=$ $\oplus_{\gamma} \mathcal{H}_{\gamma}^{\text {crit }}$ as well as the corresponding graded space $\mathcal{H}_{V}^{\text {crit }}$ associated with a sector $V$ in the upper-half plane. Hence, we have the corresponding critical DT-series $A_{V}^{\text {crit }}$ as well as critical motivic DT-invariants $\Omega^{\text {crit,mot }}(\gamma)$. In this subsection, we would like to compare $A_{V}^{\text {crit }}$ with the series $A_{V}^{\text {mot }}$ introduced in [33].

We will be sketchy. We continue to use the notation introduced in Section 7.1. We will also assume that the reader is familiar with the setup of the paper [33].

Let us make a simplifying assumption ${ }^{18}$ that $R / \mathbf{k}$ is the path algebra of a finite quiver $Q$ with the set of vertices $I$. Also we assume that the ground field is algebraically closed, $\mathbf{k}=\overline{\mathbf{k}}$. Then the potential $W$ gives rise to a homologically smooth dg $3 C Y$-algebra $B / \mathbf{k}$ with generators in degrees $-2,-1,0$ (Ginzburg algebra), and we can consider triangulated dg-category $\mathcal{C}=\mathcal{C}_{(Q, W)}$ of finite-dimensional (over $\mathbf{k}$ ) $A_{\infty}$-modules over $B$. It is an indconstructible triangulated three-dimensional Calabi-Yau k-linear category in the sense of [33]. It has a canonical bounded $t$-structure with the heart equivalent to the abelian category $($ Crit $W)(\mathbf{k})$ in the notation from Section 7.1. Define $\mathcal{C}^{\text {sp }}$ to be the full triangulated subcategory of $\mathcal{C}$ generated by objects from $\sqcup_{\gamma} \mathrm{M}_{\gamma}^{\text {sp }}(\mathbf{k})$. It is also an ind-constructible triangulated category. Any choice of a central charge $Z: \mathbb{Z}^{I} \rightarrow \mathbb{C}$ as in Section 5.1 gives an indconstructible stability condition on $\mathcal{C}^{\text {sp }}$.

Calabi-Yau category $\mathcal{C}^{\text {sp }}$ has a canonical orientation data in the sense of [33], given by the super line

$$
\operatorname{sdet}\left(\operatorname{Ext}_{R-\bmod }^{\bullet}\left(E_{1}, E_{2}\right)\right)
$$

\footnotetext{
${ }^{18}$ All the conclusion of this section hold in the general case of $I$-bigraded smooth algebra.
} 
associated with any two objects $E_{1}, E_{2} \in \mathcal{C}^{\text {sp }}$. Here we us the fact that $R$ is a subalgebra of $B$.

Let us fix a central charge $Z$ and a sector $V$ in the upper-half plane. Then, as we recalled above, the theory developed in [33] gives rise to a series $A_{V}^{\text {mot }}$. This is a series in (a completion of) the quantum torus with coefficients lying in a localization of the Grothendieck ring $\mathcal{M}^{\mu}(\operatorname{Spec}(\mathbf{k}))$ of varieties over $\mathbf{k}$ endowed with a good action of the pro-algebraic group $\mu=\lim _{n} \mu_{n}$ where $\mu_{n}$ is the finite group scheme of $n$th roots of one. For $\mathbf{k}=\mathbb{C}$ there is a natural homomorphism of rings

$$
\Phi: \mathcal{M}^{\mu}(\operatorname{Spec}(\mathbb{C})) \rightarrow K_{0}\left(M M H S_{\mathbb{A}_{\mathbb{C}}^{1}}\right)
$$

which associates with $X$ (endowed with a $\mu_{n}$-action for some $n \geqslant 1$ ) the $K$-theory class of the following object in $D^{b}\left(M H M_{\mathbb{A}_{\mathbb{C}}^{1}}\right)$ :

$$
\left(\mathbb{G}_{m, \mathbb{C}} \rightarrow \mathbb{A}_{\mathbb{C}}^{1}\right) !\left(\left(X \times \mathbb{G}_{m, \mathbb{C}}\right) / \mu_{n} \stackrel{g}{\rightarrow} \mathbb{G}_{m, \mathbb{C}}\right) ! \mathbb{Q}_{\left(X \times \mathbb{G}_{m, \mathbb{C}}\right) / \mu_{n}}(0),
$$

where the action of $\mu_{n}$ on $X \times \mathbb{G}_{m, \mathbb{C}}$ is the product of the given action on $X$ and the multiplication on $\mathbb{G}_{m}, \mathbb{C}$. Map $g$ is given by $g(x, t)=t^{n}$.

One can check that $\Phi$ maps the motivic Milnor fiber used in [33] to the class of cohomology with compact support of the sheaf of vanishing cycles. Therefore, we have proved the following result.

Proposition 7.1. One has an equality of generating series with coefficients in $K_{0}\left(M M H S_{\mathbb{A}_{\mathbb{C}}^{1}}\right)$ :

$$
\Phi\left(A_{V}^{\mathrm{mot}}\right)=D\left(A_{V}^{\mathrm{crit}}\right)
$$

where $D$ is the duality.

The above proposition ensures that the theories developed in [33] and in the present paper give essentially the same result at the level of generating series.

\section{Some speculations}

There are plenty of conjectures and speculations about Cohomological Hall algebra and related motivic DT-series. From that variety we have chosen just two. 


\subsection{Categorification of the critical COHA}

Let us keep the setup of Section 7.4. The critical cohomology $H_{\mathrm{c}}^{\bullet}$,crit $\left(X^{\mathrm{sp}}, f\right)$ are related to a certain two-periodic triangulated category. Namely, for any Zariski closed subset $X^{\mathrm{sp}} \subset f^{-1}(0)$ let us define the category of matrix factorizations supported on $X^{\mathrm{sp}}$ in the following way:

$$
\operatorname{MF}_{X^{\mathrm{sp}}}(f):=D_{X^{\mathrm{sp}}}^{b}\left(\operatorname{Coh}\left(f^{-1}(0)\right)\right) / \operatorname{Perf}_{X^{\mathrm{sp}}}\left(f^{-1}(0)\right)
$$

where the subscript $X^{\text {sp }}$ denotes the category of bounded complexes of coherent sheaves on the closed subscheme $f^{-1}(0)$ (resp. of perfect complexes on $\left.f^{-1}(0)\right)$, with cohomology sheaves supported on the closed subset $X^{\mathrm{sp}}$. Then there is a Chern character homomorphism

$$
\operatorname{ch}: K_{0}\left(\mathrm{MF}_{X^{\mathrm{sp}}}(f)\right) \rightarrow\left(H_{\mathrm{c}}^{e v, \operatorname{crit}}\left(X^{\mathrm{sp}}, f\right)\right)^{\vee}
$$

where the RHS in de Rham realization is (hypothetically) the periodic cyclic homology of $\mathrm{MF}_{X^{\text {sp }}}(f)$. One has also an equivariant version $\mathrm{MF}_{X^{\mathrm{sp}}, G}(f)$ of the above category and of the Chern character (here $G$ is an algebraic group acting on $X$ and preserving $X^{\text {sp }}$ and $f$ ). We expect that the multiplication on the critical COHA comes from a monoidal structure on the direct sum of categories

$$
\bigoplus_{\gamma \in \mathbb{Z}_{\geqslant 0}^{I}} \bigoplus_{z \in \mathbb{C}} \mathrm{MF}_{\mathrm{M}_{\gamma}^{\mathrm{sp}} \cap\left(W_{\gamma}\right)^{-1}(z), \mathrm{G}_{\gamma}}\left(W_{\gamma}-z\right) .
$$

The correspondences $\mathrm{M}_{\gamma_{1}, \gamma_{2}}$ should be upgraded to functors between different summands, and the multiplication in the critical COHA will be the induced morphism of periodic cycllic homology spaces. The monoidal structure could be thought of as a categorification of the critical COHA (which is itself a categorification of DT-invariants).

\subsection{Invariants of three-dimensional manifolds}

As we already mentioned in this paper, the comparison with [33] suggests that there exists a critical COHA associated with any ind-constructible $3 C Y$ category endowed with orientation data. The potential in this case is a (partially) formal function. Any compact oriented three-dimensional $C^{\infty}$ manifold $X$ gives an example. Namely, let us consider the triangulated category $D_{\text {constr }}^{b}(X)$ of complexes of sheaves with locally constant cohomology of finite rank. This category has a $t$-structure with the heart equivalent to the category of finite-dimensional complex representations of the fundamental group 
$\pi_{1}\left(X, x_{0}\right), x_{0} \in X$. For a given $n \geqslant 0$ the $\operatorname{stack} \operatorname{Rep}_{n}(X)$ of representations of dimension $n$ is an Artin stack of finite type over $\mathbb{C}$. Locally (in analytic topology) we can represent $\operatorname{Rep}_{n}(X)$ as the set of critical points of the Chern-Simons functional:

$$
\operatorname{CS}(A)=\int_{X} \operatorname{Tr}\left(\frac{d A \cdot A}{2}+\frac{A^{3}}{3}\right) \in \mathbb{C} /(2 \pi \mathrm{i})^{2} \mathbb{Z}
$$

where $A \in \Omega^{1}(X) \otimes \operatorname{Mat}(n, \mathbb{C})$, modulo action of the gauge group. It looks plausible that the corresponding $3 C Y$ category admits orientation data in the sense of [33]. Therefore, we obtain a topological invariant of $X$ given by the motivic DT-series in one variable. For $X=S^{3}$ the invariant coincides with the motivic DT-series for the quiver $A_{1}=Q_{0}$ endowed with the trivial potential (essentially it is the quantum dilogarithm; see Section 2.5). For $X=\left(S^{1}\right)^{3}$, it is given by Proposition 5.1 from Section 5.6 (quantum MacMahon function). One can also compute the invariant, e.g., for $X=S^{1} \times S^{2}$, but in general the answer is not known to us.

Another interesting story is a relation of COHA with the holomorphic Chern-Simons functional for $\bar{\partial}$-connections on $C^{\infty}$ complex vector bundles on a compact complex $3 C Y$ manifold $X_{\mathbb{C}}$ endowed with a non-zero holomorphic 3 form $\Omega_{X_{C}}^{3,0}$. In this case, $C S$ is defined modulo the abelian subgroup of $\mathbb{C}$ consisting (up to a torsion) of integrals of $\Omega_{X_{\mathbb{C}}}^{3,0}$ over integral cycles.

Also for both $C^{\infty}$ and holomorphic Chern-Simons functionals one can try to define a rapid decay version of COHA. The latter is achieved by taking into account Stokes data (the same as gluing data in [30]) and counting gradient lines of the real part of $\exp (\mathrm{i} \phi) C S$ for various $\phi \in \mathbb{R} / 2 \pi \mathbb{Z}$, which connect different critical points of $C S$. In order to do this we have to use an appropriate infinite covering of the space of connections, in order to have a globally defined holomorphic functional $C S$. This goes beyond the formalism of $3 C Y$ categories, as the gradient lines are trajectories in the space of non-flat connection in the case of a real oriented three-dimensional manifold, or non-holomorphic $\bar{\partial}$-connections in the complex case. Geometrically these lines correspond to self-dual non-unitary connections on the four-dimensional Riemannian manifold $X \times \mathbb{R}$ in the real case, or on the Spin(7)-manifold $X_{\mathbb{C}} \times \mathbb{R}$ in the complex case, with appropriate boundary conditions at infinity (compare with [55]). The resulting structure is in a sense an exponential mixed Hodge structure of infinite rank. We hope to discuss it in the future. 


\section{Acknowledgments}

We thank to Pierre Deligne, Michael Douglas, Davide Gaiotto, Ian Grojnowski, Greg Moore, Nikita Nekrasov, Andrei Okounkov, Joerg Schürmann for useful conversations and communications. Also we are grateful to referees for several remarks and corrections. We thank to Morihiko Saito for sending us a preliminary version of [50]. This work was started during the workshop at Simons Center for Geometry and Physics in January, 2008. Y.S. thanks to the University of Miami and IHES for the hospitality. His work was partially supported by an NSF grant.

\section{References}

[1] M. Barr, Harrison homology, Hochschild homology and triples, J. Algebra 8 (1968), 314-323.

[2] K. Behrend, On the de Rham cohomology of differential and algebraic stacks, arXiv:math/0410255.

[3] K. Behrend, J. Bryan and B. Szendroi, Motivic degree zero DonaldsonThomas invariants, arXiv:0909.5088.

[4] S. Barannikov, Matrix De Rham complex and quantum A-infinity algebras, arXiv:1001.5264.

[5] A. Beilinson and V. Vologodsky, A DG guide to Voevodsky's motives, arXiv:math/0604004.

[6] V. Berkovich, Vanishing cycles for formal schemes, Invent. Math. 115(3) (1994), 539-571.

[7] V. Berkovich, Vanishing cycles for formal schemes II, Invent. Math. 125(2) (1996), 367-390.

[8] J. Bernstein and V. Lunts, Equivariant sheaves and functors. Lecture notes in mathematics, 1578, Springer-Verlag, 1994.

[9] M. Bondarko, Differential graded motives: weight complex, weight filtrations and spectral sequences for realizations; Voevodsky versus Hanamura, J. Inst. Math. Jussieu 8(1) (2009), 39-97, arXiv:math/0601713.

[10] J. Cuntz and D. Quillen, Algebra extensions and nonsingularity, J. Amer. Math. Soc. 8 (1995), 251-289. 
[11] J. Denef and F. Loeser, Motivic exponential integrals and a motivic Thom-Sebastiani Theorem, Duke Math. J. 99(2) (1999), 285-290, arXiv:math/9803048.

[12] A. Dimca, Sheaves in topology, Universitext, Springer-Verlag, 2004.

[13] A. Dimca and B. Szendroi, The Milnor fibre of the Pfaffian and the Hilbert scheme of four points on $\mathbb{C}^{3}$, Math. Res. Lett. 16 (2009), 10371055, arXiv:0904.2419.

[14] A. Efimov, Cohomological Hall algebra of a symmetric quiver, arXiv:1103:2736.

[15] B. Enriquez, Quantization of Lie bialgebras and shuffle algebras of Lie algebras, arXiv:math/0101059.

[16] B. Eynard, Formal matrix integrals and combinatorics of maps, arXiv:math-ph/0611087.

[17] B. Feigin and A. Odesskii, Vector bundles on elliptic curve and Sklyanin algebras, arXiv:q-alg/9509021.

[18] V. Fock and A. Goncharov, Cluster ensembles, quantization and the dilogarithm, arXiv:math/0311245.

[19] S. Fomin and A. Zelevinsky, Cluster algebras: Notes for the CDM-03 conference, arXiv:math/0311493.

[20] L. Fu, A Thom-Sebastiani Theorem in characteristic p, arXiv:1105.5201.

[21] D. Gaiotto, G. Moore and A. Neitzke, Framed BPS States, arXiv:1006.0146.

[22] E. Getzler and M. Kapranov, Cyclic operads and cyclic homology, in Geometry, Topology and Physics for R. Bott, ed. S.-T. Yau, pp. 167201, International Press, Cambridge MA, 1995.

[23] I. Grojnowski, Affinizing quantum algebras: from $\mathcal{D}$-modules to $K$ theory, preprint (1994).

[24] M. Gross, R. Pandharipande and B. Siebert, The tropical vertex, arXiv:0902.0779.

[25] A. Grothendieck, La theorie des classes de Chern, Bull. Soc. Math. France, 86 (1958), 137-154. 
[26] J. A. Harvey and G. Moore, On the algebras of BPS states, Comm. Math. Phys. 197(3) (1998), 489-519, and arXiv:hep-th/9609017.

[27] Yunfeng Jiang, Motivic Milnor fiber of cyclic L-infinity algebras, arXiv:0909.2858.

[28] D. Joyce and Y. Song, A theory of generalized Donaldson-Thomas invariants, arXiv:0810.5645.

[29] N. Katz, Exponential sums, Ann. Math. Study 124, Princeton University Press, Princeton, NJ, 1990.

[30] L. Katzarkov, M. Kontsevich and T. Pantev, Hodge theoretic aspects of mirror symmetry, in Proceedings of Symposia in Pure Mathematics Vol. 78 (2008), "From Hodge theory to integrability and TQFT: tt*geometry", eds. Ron Y. Donagi and Katrin Wendland, pp. 87-174, arXiv:0806.0107.

[31] D. Knutson, $\lambda$-rings and the representation theory of the symmetric group, Lecture notes in Mathematics, 308, 1973.

[32] M. Kontsevich and A. Rosenberg, Noncommutative smooth spaces, arXiv:math/9812158.

[33] M. Kontsevich and Y. Soibelman, Stability structures, motivic Donaldson-Thomas invariants and cluster transformations, arXiv:0811.2435.

[34] M. Kontsevich and Y. Soibelman, Affine structures and nonarchimedean analytic spaces, arXiv:math/0406564.

[35] M. Kontsevich and D. Zagier, Periods, in Mathematics Unlimited 2001 and Beyond. eds. B. Engquist and W. Schmid, pp. 771-808, Springer, 2000.

[36] V. Kulikov, Mixed Hodge Structures and Singularities, Cambridge Tracts in Mathematics, 132, Cambridge University Press, Cambridge, 1998.

[37] A. Losev, G. Moore, N. Nekrasov and S. Shatashvili, Four-Dimensional Avatars of Two-Dimensional RCFT, arXiv:hep-th/9509151.

[38] Y. Laszlo and M. Olson, The six operations for sheaves on Artin stacks I,II. Publ. IHES, 107 (2008), 109-210.

[39] G. Lusztig, Quivers, Perverse Sheaves, and Quantized Enveloping Algebras, J. Amer. Math. Soc. 4(2) (1991), 365-421. 
[40] I. Macdonald, Symmetric Functions and Hall Polynomials, Oxford University Press, 1995.

[41] B. Malgrange, Équations différentielles à coefficients polynomiaux, Progress in Math. 69, Birkháuser, Basel, Boston, 1991.

[42] D. B. Massey, The Sebastiani-Thom isomorphism in the Derived Category, Comput. Math. 125(3) (2001), 353-362.

[43] H. Nakajima, Instantons and affine Lie algebras, in S-duality and mirror symmetry, Nucl. Phys. B (Proc. Suppl.), 46 (1996), 154-161, arXiv:alg-geom/9510003.

[44] A. Némethi and C. Sabbah, Semicontinuity of the spectrum at infinity, J. Abhandlungen aus dem Mathematischen Seminar der Universitt Hamburg, 69(1) (1999), 25-35, arXiv:math/9805086.

[45] J. Nicaise and J. Sebag, Motivic Serre invariants, ramification, and the analytic Milnor fiber, Invent. Math. 168(1) (2007), 133-173.

[46] C. Peters and J. Steenbrink, Mixed Hodge structures, Ergebnisse der Mathematik und ihrer Grenzgebiete, 52, Springer-Verlag, Berlin, 2008.

[47] M. Reineke, Cohomology of quiver moduli, functional equations, and integrality of Donaldson-Thomas type invariants, arXiv:0903.0261.

[48] C. Sabbah, On the comparison theorem for elementary irregular D-modules, Nagoya Math. J. 141 (1996), 107-124.

[49] M. Saito, Introduction to mixed Hodge modules, Astérisque, 179-180 (1989), 145-162.

[50] M. Saito, Thom-Sebastiani theorem for Hodge modules, preprint Kyoto University, 2010.

[51] J. Schürmann, Topology of singular spaces and constructible sheaves, Monografie Matematyczne, 63, Birkhäuser Verlag, Basel, 2003.

[52] SGA 7, vol.2, Lecture Notes in Mathematics, 340, Springer-Verlag, Berlin, New York, 1973.

[53] M. Temkin, Absolute desingularization in characteristic zero, arXiv:1001.5433.

[54] V. Voevodsky, Triangulated category of motives, in Cycles, transfers and motivic homology theories (ed. V. Voevodsky, A. Suslin and 
E. Friedlander), Annals of Mathematical Studies, 143, pp. 188-238, Princeton University Press, 2000.

[55] E. Witten, Analytic Continuation Of Chern-Simons Theory, arXiv:1001.2933.

[56] A. Zelevinsky, Mutations for quivers with potentials: Oberwolfach talk, April 2007, arXiv:0706.0822.

[57] Zhu Bin, Applications of BGP-reflection functors: isomorphisms of cluster algebras, Science in China Series A: Mathematics, 49(12) (2006), 1839-1854.

\section{IHES}

35 Route De Chartres

F-91440

FRANCE

E-mail address: maxim@ihes.fr

Department of Mathematics

KSU

MANHATtAN, KS 66506

USA

E-mail address: soibel@math.ksu.edu

ReCEIVEd OCtOBer 14, 2010 